\title{
Multicomponent, Tandem 1,3- and 1,4-Bisarylation of Donor- Acceptor Cyclopropanes and Cyclobutanes with Electron-Rich Arenes and Hypervalent Arylbismuth Reagents
}

Biplab Mondal $^{\dagger}$, Dinabandhu Das ${ }^{\ddagger}$ and Jaideep Saha ${ }^{\dagger}$

${ }^{\dagger}$ Division of Molecular Synthesis and Drug Discovery, Centre of Biomedical Research (CBMR), SGPGIMS Campus, Lucknow 226014, Uttar Pradesh. India.

${ }^{\ddagger}$ School of Physical Sciences, Jawaharlal Nehru University, New Delhi-110067, India.

\section{Table of Contents}

\begin{tabular}{|c|c|c|}
\hline Entry & Description & Page \\
\hline \multirow{2}{*}{1} & General Experimental... & 2 \\
\hline & Detailed Optimization Conditions of the Reaction..... & 3 \\
\hline 2 & 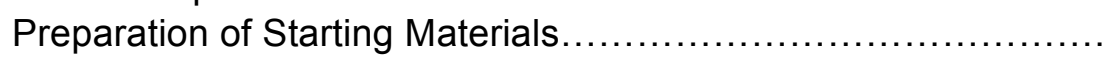 & $4-5$ \\
\hline \multirow[t]{2}{*}{3} & General Procedure for the Preparation of Compounds $\mathbf{4}$ and & \\
\hline & 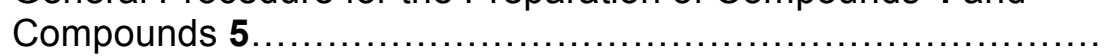 & 6 \\
\hline 4 & 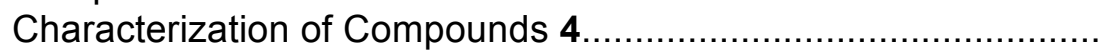 & $7-17$ \\
\hline 6 & Characterization of Compounds $5 \ldots \ldots \ldots \ldots \ldots \ldots \ldots \ldots \ldots \ldots$ & $18-27$ \\
\hline 7 & General Procedure for the Preparation of Compounds $7 \ldots \ldots \ldots \ldots \ldots$ & 28 \\
\hline 8 & 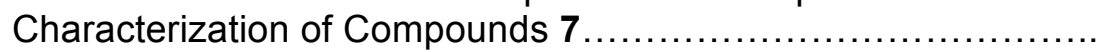 & 29-32 \\
\hline 9 & Synthesis and Characterization Data of Compounds $8 \ldots$. & $33-34$ \\
\hline 10 & 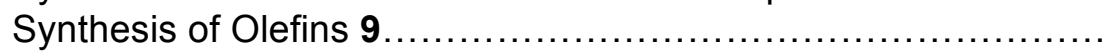 & $35-36$ \\
\hline 11 & HPLC Data for Enatiospecific Synthesis........................... & 37 \\
\hline 13 & NMR Spectra of New Compounds................................... & $38-93$ \\
\hline 14 & 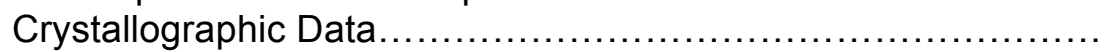 & $94-96$ \\
\hline 15 & 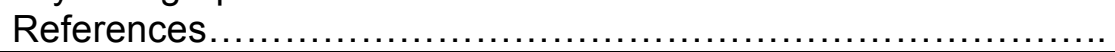 & 97 \\
\hline
\end{tabular}




\section{General Experimental.}

Unless otherwise mentioned, all the new reactions in the study were performed maintaining an inert atmosphere (nitrogen or argon) and flame-dried glassware were used. Solvents were dried before use following standard procedures and only freshly distilled THF has been used. Unless noted, all the reagents and catalysts were used as it was received from commercial sources and no further purification was made on those. Reaction monitoring was performed via TLC, using Merck silica gel $60 \mathrm{~F} 254$ plates. TLC plates were visualized either under UV light $(254 \mathrm{~nm})$ or by using $10 \%$ ethanolic phosphomolybdic acid (PMA) or $1 \%$ aqueous $\mathrm{KMnO}_{4}$ or iodine. Silica gel of 230-400 mesh size was used for the flash column chromatography. ${ }^{1} \mathrm{H},{ }^{13} \mathrm{C}$ NMR spectra were recorded on Avance III, Bruker at $400 \mathrm{MHz}, 100 \mathrm{MHz}$ and $376 \mathrm{MHz}$ spectrometers respectively using $\mathrm{CDCl}_{3}$. In the experimental section, the ${ }^{1} \mathrm{H} \mathrm{NMR}$ chemicals shift are expressed in the form of ppm $(\delta)$ relative to $\delta=7.26$ for $\mathrm{CDCl}_{3}$ whereas ${ }^{13} \mathrm{C}$ NMR chemical shift are expressed relative to $\delta=77.16$. HRMS and Electron Spray Ionization (ESI) (m/z) spectra were recorded on Agilent Technologies 6530 Accurate- Mass Q-TOF LC/MS. Enantiomeric excess (ee) was measured by HPLC analysis with chiral stationary phase. FT-IR experiments were performed on PerkinElmer Spectrum Version 10.03.08. 


\section{Detailed Optimization of the Reaction Conditions (Table S1).}

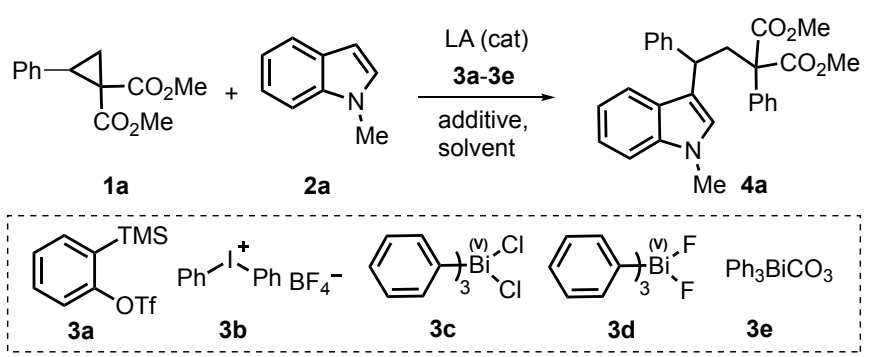

\begin{tabular}{|c|c|c|c|c|c|c|}
\hline Entry & Cat. & $\mathrm{mol} \%$ & $\mathrm{Ar}^{+}$source & $\begin{array}{l}\text { Base/ } \\
\text { additive }\end{array}$ & Solvent & ${\text { Yield }[\%]^{b}}^{b}$ \\
\hline $1^{c}$ & $\mathrm{Yb}(\mathrm{OTf})_{3}$ & 10 & $3 a$ & CsF & $\mathrm{CH}_{3} \mathrm{CN}$ & 0 \\
\hline $2^{c}$ & $\mathrm{Sc}(\mathrm{OTf})_{3}$ & 10 & $3 a$ & $\mathrm{CsF}$ & $\mathrm{CH}_{3} \mathrm{CN}$ & 0 \\
\hline $3^{c}$ & $\mathrm{Sc}(\mathrm{OTf})_{3}$ & 10 & $3 a$ & $\mathrm{CsF}$ & $\mathrm{Et}_{2} \mathrm{O}$ & 0 \\
\hline 4 & $\mathrm{Sc}(\mathrm{OTf})_{3}$ & 10 & $3 b$ & tBuOK & DCM & $\mathrm{ND}^{d}$ \\
\hline 5 & $\mathrm{Yb}(\mathrm{OTf})_{3}$ & 10 & $3 c$ & TMG & DCM & 30 \\
\hline 6 & $\mathrm{Sc}(\mathrm{OTf})_{3}$ & 10 & $3 c$ & TMG & DCM & 61 \\
\hline $7^{e}$ & $\mathrm{Sc}(\mathrm{OTf})_{3}$ & 10 & $3 c$ & - & DCM & 0 \\
\hline 8 & $\mathrm{Sc}(\mathrm{OTf})_{3}$ & 10 & $3 c$ & TEA & DCM & $<10$ \\
\hline 9 & $\mathrm{Sc}(\mathrm{OTf})_{3}$ & 10 & $3 c$ & DBU & DCM & 45 \\
\hline 10 & $\mathrm{Sc}(\mathrm{OTf})_{3}$ & 10 & $3 c$ & ${ }^{t} \mathrm{BuOK}$ & DCM & 84 \\
\hline 11 & $\mathrm{Sc}(\mathrm{OTf})_{3}$ & 10 & $3 c$ & ${ }^{t} \mathrm{BuOK}$ & THF & 22 \\
\hline 12 & $\mathrm{Sc}(\mathrm{OTf})_{3}$ & 10 & $3 c$ & ${ }^{t} \mathrm{BuOK}$ & Toluene & 55 \\
\hline 13 & $\mathrm{Sc}(\mathrm{OTf})_{3}$ & 10 & $3 c$ & ${ }^{t} \mathrm{BuOK}$ & $\mathrm{ACN}$ & 46 \\
\hline 14 & $\mathrm{Sc}(\mathrm{OTf})_{3}$ & 10 & $3 c$ & ${ }^{t} \mathrm{BuOK}$ & $\mathrm{Et}_{2} \mathrm{O}$ & 91 \\
\hline 15 & $\mathrm{Yb}(\mathrm{OTf})_{3}$ & 10 & $3 c$ & ${ }^{t} \mathrm{BuOK}$ & $\mathrm{Et}_{2} \mathrm{O}$ & 55 \\
\hline 16 & $\ln (\mathrm{OTf})_{3}$ & 10 & $3 c$ & ${ }^{t} \mathrm{BuOK}$ & $\mathrm{Et}_{2} \mathrm{O}$ & $\mathrm{ND}^{d}$ \\
\hline 17 & $\mathrm{Sn}(\mathrm{OTf})_{2}$ & 10 & $3 c$ & ${ }^{t} \mathrm{BuOK}$ & $\mathrm{Et}_{2} \mathrm{O}$ & 10 \\
\hline 18 & $\mathrm{Ni}\left(\mathrm{ClO}_{4}\right)_{2}$ & 10 & $3 c$ & ${ }^{t} \mathrm{BuOK}$ & $\mathrm{Et}_{2} \mathrm{O}$ & 31 \\
\hline 19 & $\mathrm{Sc}(\mathrm{OTf})_{3}$ & 10 & 3d & ${ }^{t} \mathrm{BuOK}$ & $\mathrm{Et}_{2} \mathrm{O}$ & 83 \\
\hline $20^{c}$ & $\mathrm{Sc}(\mathrm{OTf})_{3}$ & 10 & $3 e$ & ${ }^{t} \mathrm{BuOK}$ & $\mathrm{Et}_{2} \mathrm{O}$ & 0 \\
\hline 21 & $\mathrm{Sc}(\mathrm{OTf})_{3}$ & 20 & $3 c$ & ${ }^{t} \mathrm{BuOK}$ & $\mathrm{Et}_{2} \mathrm{O}$ & 70 \\
\hline $22^{f}$ & $\mathrm{Sc}(\mathrm{OTf})_{3}$ & 10 & $3 c$ & ${ }^{t} \mathrm{BuOK}$ & $\mathrm{Et}_{2} \mathrm{O}$ & 65 \\
\hline
\end{tabular}

${ }^{a}$ Reaction conditions: 1a (1.0 equiv), 2a (1.5 equiv), 3a-3e (1.25 equiv), conc [0.1 M], rt. ${ }^{b}$ Isolated yield of $\mathbf{4 a}$. ${ }^{c}$ Indole addition product to D-A cyclopropane along with formation of unknown products could be traced from the reaction mixture. ${ }^{d} \mathrm{ND}=$ Not Determined; product mass was observed by MS in this case. ${ }^{e}$ Without base, no product formation was observed. ${ }^{f}$ Reaction was performed with [0.2 M] concentration. 


\section{Preparation of the starting materials}

(a) Donor-Acceptor Cyclopropanes: Following donor-acceptor cyclopropanes were used in the study and were prepared following the literature procedures. ${ }^{1}$
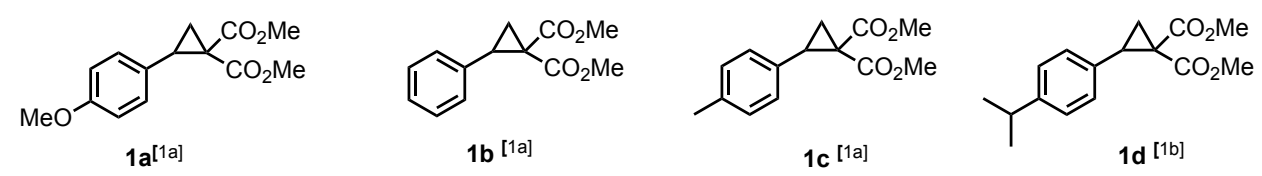<smiles>COCC1(OC)CC1c1ccc(C(C)(C)C)cc1</smiles><smiles>CCOC(=O)C1(c2ccc(OC)cc2)CC1OCC</smiles><smiles>COc1ccc(C2CC2(C(C)=O)C(C)=O)cc1OC</smiles><smiles>COC(=O)C1CC1c1cc(OC)c(OC)c(OC)c1</smiles><smiles>COC(=O)C1(C(=O)OC)CC1c1ccc(F)cc1</smiles>

$1 \mathrm{~m}^{[1 \mathrm{~b}]}$<smiles>COC(C)C1CC1c1cccc2ccccc12</smiles>

$1 q^{[1 b]}$<smiles>CC(=O)OC1(C(C)=O)CC1N1C(=O)c2ccccc2C1=O</smiles>

$1 u^{[1 b, f]}$

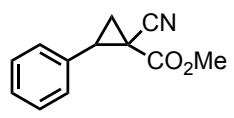

1y ${ }^{[1]}$<smiles>CCOC(OCC)(C1CC1c1ccc(OC)cc1)C(OC)C1CC1c1cccc(OC)c1</smiles><smiles>COC(=O)C1(C(C)=O)CC1c1ccccc1C</smiles>
$1 k^{[1]]}$<smiles>COc1ccccc1C1CC1C(C)=O</smiles>
$1]^{[1 \mathrm{~b}]}$<smiles>CC(=O)OC1(C(C)=O)CC1c1ccc(Cl)cc1</smiles>

1n [1a]<smiles>COC(=O)C1(C(C)=O)CC1c1ccc2ccccc2c1</smiles>
$1 r^{[1]]}$<smiles>CC(=O)OC1(C(C)=O)CC1c1ccc(Br)cc1</smiles>

10 ${ }^{[11]}$<smiles>CC(=O)OC1(C(C)=O)CC1c1ccccc1Br</smiles>

$1 p^{[19]}$<smiles>COC(=O)C1(C(C)=O)CC1c1ccco1</smiles>

$1 \mathrm{~s}^{[1 \mathrm{~b}]}$<smiles>COC(OC)C1CC1c1cccs1</smiles>

$1 t^{[1 b, f]}$<smiles>O=C(c1ccccc1)C1(C(=O)c2ccccc2)CC1c1ccccc1</smiles>

$1 w^{[1 b]}$<smiles>CCOC(=O)C1CC1c1ccccc1</smiles>

$1 x^{[1]}$

Figure S1: List of Donor-Acceptor (D-A) cyclopropanes used in the study.

(b) Donor-Acceptor Cyclobutanes: Following D-A cyclobutanes were used in the study and prepared according to the literature method. ${ }^{2}$<smiles>COC(=O)C1(C(C)=O)CCC1c1ccc(OC)cc1</smiles>

6a<smiles>COC(=O)C1(C(C)=O)CCC1c1ccc(OCc2ccccc2)cc1</smiles>

6b

Figure S2: Donor-acceptor (D-A) cyclobutanes used in the study 
(c) Triaryl bismuth (V) reagents: Different triarylbismuth (V) reagents of the following structures were prepared according to the literature method. ${ }^{3}$
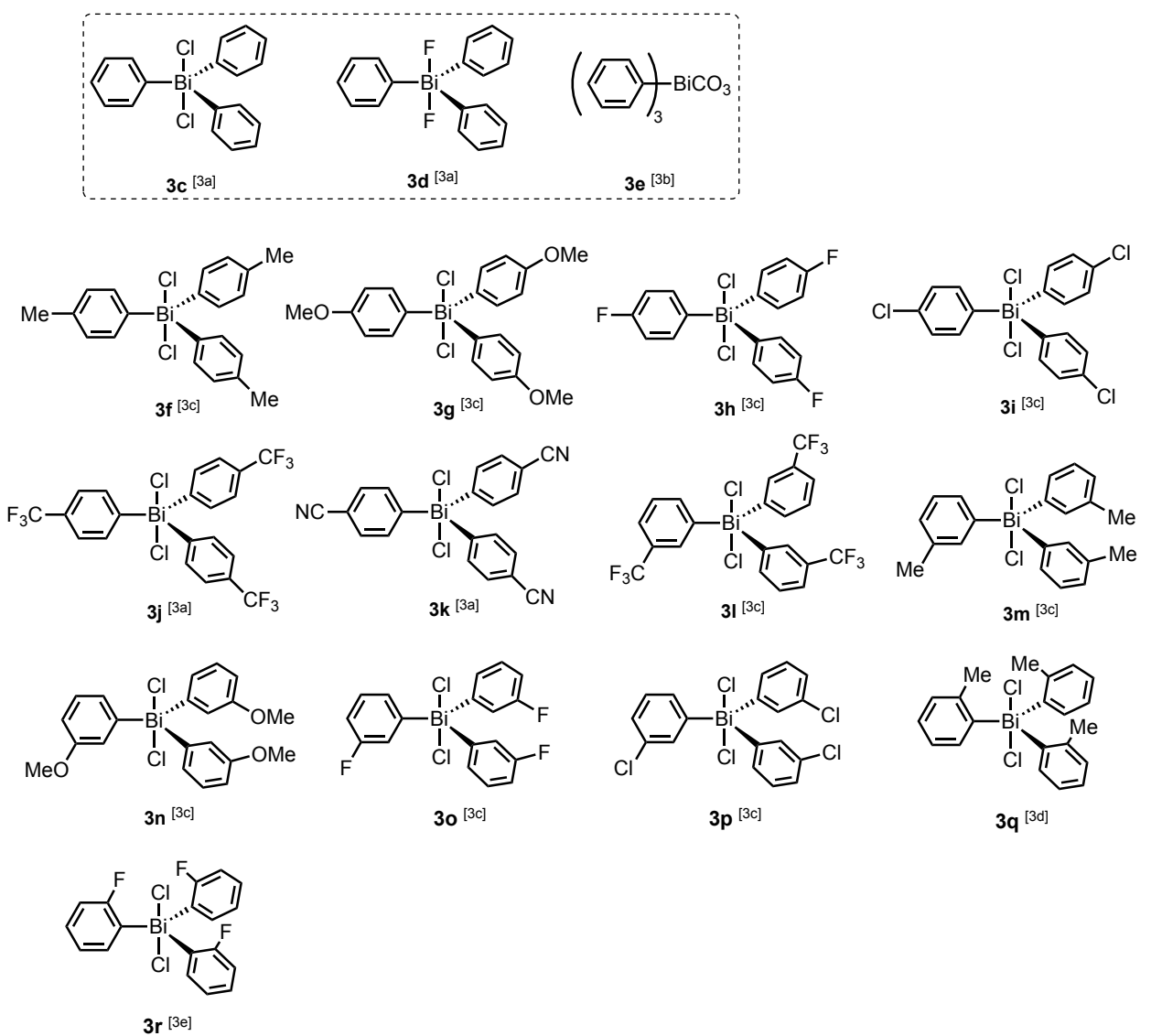

Figure S3: Structure of different triarylbismuth(V) compounds. (400 MHz, CDCl3) 


\section{General Procedure for the preparation of compounds 4 and 5}

$$
\underbrace{-C_{2} \mathrm{CO}_{2} \mathrm{R}^{2}}_{3}+\mathrm{FG} \frac{\mathrm{Ar}}{2}
$$

Under $\mathrm{N}_{2}$ atmosphere, donor-Acceptor (D-A) cyclopropane (1.0 equiv), arene (1.5 equiv.) and $\mathrm{Sc}(\mathrm{OTf})_{3}(10 \mathrm{~mol} \%)$ were taken in anhydrous diethyl ether $(0.1 \mathrm{M})$. To this solution were successively added ${ }^{\mathrm{t}} \mathrm{BuOK}$ (2.0 equiv) and $\mathrm{Ar}_{3} \mathrm{BiCl}_{2}$ (1.25 equiv) in one portion and the reaction mixture was stirred at room temperature until completion of the reaction as determined by TLC analysis (ca. 4-10 h). When the starting material was consumed, the crude production was purified by column chromatography on silica gel to provide the pure product. 


\section{Preparation and characterization data of compounds 4}

Dimethyl 2-(2-(4-methoxyphenyl)-2-(1-methyl-1H-indol-3-yl)etyl)-2phenylmalonate $(4 a)$ :<smiles>COc1ccc(C(CC(C)(OC)c2ccccc2)c2cn(C)c3ccccc23)cc1</smiles>

Following the general procedure, D-A cyclopropane $(1 \mathrm{a}, 0.027 \mathrm{~g}, 0.1 \mathrm{mmol}$ ) was subjected to 1,3-bisarylation using 1-methyl indole and $\mathrm{Ph}_{3} \mathrm{BiCl}_{2}$ as arene sources and the crude product was purified via silica gel column chromatography (EtOAc: Hexane $3: 7)$, which afforded compound $\mathbf{4 a}$ in $91 \%$ yield $(0.043 \mathrm{~g})$ as white solid. $\mathbf{m p :}$ 135-138 ${ }^{\circ} \mathrm{C} ; R_{f} 0.20$ (EtOAc: Hexane 3:7); ${ }^{1} \mathrm{H}$ NMR $\left(\mathrm{CDCl}_{3}, 400 \mathrm{MHz}\right) \delta 7.58$ (d, $\mathrm{J}=$ $7.3 \mathrm{~Hz}, 2 \mathrm{H}), 7.36-7.28(\mathrm{~m}, 4 \mathrm{H}), 7.23-7.20(\mathrm{~m}, 3 \mathrm{H}), 7.15(\mathrm{t}, J=7.6 \mathrm{~Hz}, 1 \mathrm{H}), 6.98(\mathrm{t}, J$ $=7.5 \mathrm{~Hz}, 1 \mathrm{H}), 6.79-6.77(\mathrm{~m}, 3 \mathrm{H}), 4.24(\mathrm{t}, J=6.5 \mathrm{~Hz}, 1 \mathrm{H}), 3.75(\mathrm{~s}, 3 \mathrm{H}), 3.70(\mathrm{~s}, 3 \mathrm{H})$, $3.37(\mathrm{~s}, 3 \mathrm{H}), 3.29-3.33(\mathrm{~m}, 4 \mathrm{H}), 3.18(\mathrm{dd}, \mathrm{J}=14.5,7.4 \mathrm{~Hz}, 1 \mathrm{H}) ;{ }^{13} \mathrm{C}\left\{{ }^{1} \mathrm{H}\right\}$ NMR $\left(\mathrm{CDCl}_{3}, 100 \mathrm{MHz}\right) \delta 171.1,170.7,157.9,137.3,137.2,136.7,129.3,128.4,128.3$, 127.7, 126.9, 126.4, 121.6, 119.8, 119.0, 118.8, 113.7, 109.1, 61.6, 55.4, 52.5, 52.3, 42.1, 37.8, 32.8; HRMS (ESI-TOF) m/z: [M+Na] ${ }^{+} \mathrm{C}_{29} \mathrm{H}_{29} \mathrm{NNaO}_{5}$ Calcd. 494.1943, Found 494.1935.

Dimethyl 2-(2-(1-methyl-1H-indol-3-yl)-2-phenylethyl)-2-phenylmalonate (4b):<smiles>COC(=O)C(CC(C)=O)(CC(c1ccccc1)c1ccccc1)c1ccccc1</smiles>

Following the general procedure, D-A cyclopropane $(1 \mathbf{b}, 0.024 \mathrm{~g}, 0.1 \mathrm{mmol})$ was subjected to 1,3-bisarylation using 1-methyl indole and $\mathrm{Ph}_{3} \mathrm{BiCl}_{2}$ as arene sources and the crude product was purified via silica gel column chromatography (EtOAc: Hexane $2: 8)$, which afforded compound $\mathbf{4 b}$ in $86 \%$ yield $(0.038 \mathrm{~g})$ as colourless oil. $R_{\mathrm{f}} 0.35$ (EtOAc: Hexane 3:7); ${ }^{1} \mathrm{H}$ NMR $\left(\mathrm{CDCl}_{3}, 400 \mathrm{MHz}\right) \delta 7.57(\mathrm{~d}, \mathrm{~J}=7.4 \mathrm{~Hz}, 2 \mathrm{H})$, 7.37 - $7.30(\mathrm{~m}, 6 \mathrm{H}), 7.25-7.20(\mathrm{~m}, 3 \mathrm{H}), 7.16-7.10(\mathrm{~m}, 2 \mathrm{H}), 6.98(\mathrm{t}, J=7.4 \mathrm{~Hz}, 1 \mathrm{H})$, $6.80(\mathrm{~s}, 1 \mathrm{H}), 4.28(\mathrm{t}, J=6.4 \mathrm{~Hz}, 1 \mathrm{H}), 3.70(\mathrm{~s}, 3 \mathrm{H}), 3.35(\mathrm{~s}, 3 \mathrm{H}), 3.31-3.19(\mathrm{~m}, 5 \mathrm{H})$; ${ }^{13} \mathrm{C}\left\{{ }^{1} \mathrm{H}\right\}$ NMR $\left(\mathrm{CDCl}_{3}, 100 \mathrm{MHz}\right) \delta 171.1,170.7,144.6,137.2,137.1,128.4$ (3), 128.3, 127.8, 126.9 126.5, 126.2, 121.6, 119.7, 118.9, 118.6, 109.2, 61.6, 52.5, 52.3, 41.9, 38.7, 32.8; HRMS (ESI-TOF) m/z: [M+Na] ${ }^{+} \mathrm{C}_{28} \mathrm{H}_{27} \mathrm{NNaO}_{4}$ Calcd. 464.1838, Found 464.1821. 


\section{Dimethyl 2-(2-(1-methyl-1H-indol-3-yl)-2-(p-tolyl)ethyl)-2-phenylmalonate (4c):}<smiles>COC(C)(OC)C(CC(c1ccccc1)c1cn(C)c2ccccc12)c1ccc(Nc2ccccc2)cc1</smiles>

Following the general procedure, D-A cyclopropane (1c, $0.025 \mathrm{~g}, 0.1 \mathrm{mmol}$ ) was subjected to 1,3-bisarylation using 1-methyl indole and $\mathrm{Ph}_{3} \mathrm{BiCl}_{2}$ as arene sources and the crude product was purified via silica gel column chromatography (EtOAc: Hexane 2:8), which afforded compound $4 \mathrm{c}$ in $86 \%$ yield $(0.039 \mathrm{~g})$ as colourless oil. $R_{f} 0.35$ (EtOAc: Hexane 3:7); ${ }^{1} \mathrm{H}$ NMR $\left(\mathrm{CDCl}_{3}, 400 \mathrm{MHz}\right) \delta 7.60$ - $7.57(\mathrm{~m}, 2 \mathrm{H}), 7.38$ $7.31(\mathrm{~m}, 4 \mathrm{H}), 7.22-7.20(\mathrm{~m}, 3 \mathrm{H}), 7.15(\mathrm{t}, J=7.5 \mathrm{~Hz}, 1 \mathrm{H}), 7.05(\mathrm{~d}, J=7.8 \mathrm{~Hz}, 2 \mathrm{H})$, $6.99(\mathrm{t}, J=7.4 \mathrm{~Hz}, 1 \mathrm{H}), 6.80(\mathrm{~s}, 1 \mathrm{H}), 4.26(\mathrm{t}, J=6.4 \mathrm{~Hz}, 1 \mathrm{H}), 3.70(\mathrm{~s}, 3 \mathrm{H}), 3.35(\mathrm{~s}$, $3 \mathrm{H}), 3.30-3.18(\mathrm{~m}, 5 \mathrm{H}), 2.28(\mathrm{~s}, 3 \mathrm{H}) ;{ }^{13} \mathrm{C}\left\{{ }^{1} \mathrm{H}\right\}$ NMR $\left(\mathrm{CDCl}_{3}, 100 \mathrm{MHz}\right) \delta 171.1$, $170.7,141.6,137.3,137.2,135.5,128.9,128.4,128.3,128.2,127.7,127.0,126.5$, $121.6,119.8,118.8,118.7,109.1,61.6,52.4,52.3,42.0,38.2,32.7,21.1$; HRMS (ESI-TOF) m/z: [M+Na] ${ }^{+} \mathrm{C}_{29} \mathrm{H}_{29} \mathrm{NNaO}_{4}$ Calcd. 478.1994, Found 478.1989.

\section{Dimethyl phenylmalonate (4d): \\ 2-(2-(4-isopropylphenyl)-2-(1-methyl-1H-indol-3-yl)ethyl)-2-}<smiles>COC(C)(CC(c1ccc(C(C)C)cc1)c1ccccc1N(C)C)c1ccccc1</smiles>

Following the general procedure, D-A cyclopropane $(1 \mathrm{~d}, 0.028 \mathrm{~g}, 0.1 \mathrm{mmol})$ was subjected to 1,3-bisarylation using 1-methyl indole and $\mathrm{Ph}_{3} \mathrm{BiCl}_{2}$ as arene sources and the crude product was purified via silica gel column chromatography (EtOAc: Hexane 3:7), which afforded compound $4 \mathbf{d}$ in $77 \%$ yield $(0.037 \mathrm{~g})$ as white solid. mp:113-116 ${ }^{\circ} \mathrm{C} ; R_{f} 0.35$ (EtOAc: Hexane 3:7); ${ }^{1} \mathrm{H}$ NMR $\left(\mathrm{CDCl}_{3}, 400 \mathrm{MHz}\right) \delta 7.57$ (d, $J=7.6 \mathrm{~Hz}, 2 \mathrm{H}), 7.38(\mathrm{~d}, J=8.0 \mathrm{~Hz}, 1 \mathrm{H}), 7.36-7.28(\mathrm{~m}, 3 \mathrm{H}), 7.24-7.20(\mathrm{~m}, 3 \mathrm{H}), 7.15$ $(\mathrm{t}, J=7.5 \mathrm{~Hz}, 1 \mathrm{H}), 7.09(\mathrm{~d}, J=8.0 \mathrm{~Hz}, 2 \mathrm{H}), 6.99(\mathrm{t}, J=7.5 \mathrm{~Hz}, 1 \mathrm{H}), 6.81(\mathrm{~s}, 1 \mathrm{H}), 4.26$ $(\mathrm{t}, J=6.4 \mathrm{~Hz}, 1 \mathrm{H}), 3.70(\mathrm{~s}, 3 \mathrm{H}), 3.34(\mathrm{~s}, 3 \mathrm{H}), 3.30-3.18(\mathrm{~m}, 5 \mathrm{H}), 2.88-2.78(\mathrm{~m}, 1 \mathrm{H})$, $1.19(\mathrm{~d}, J=6.9 \mathrm{~Hz}, 6 \mathrm{H}) ;{ }^{13} \mathrm{C}\left\{{ }^{1} \mathrm{H}\right\} \operatorname{NMR}\left(\mathrm{CDCl}_{3}, 100 \mathrm{MHz}\right) \delta 171.1,170.7,146.5$, $141.9,137.2,137.2,128.5,128.3,128.2,127.7,127.0,126.5,126.3,121.6,119.8$, 118.8, 109.1, 61.6, 52.4, 52.2, 42.1, 38.2, 33.8, 32.8, 24.2, 24.1; HRMS (ESI-TOF) $\mathrm{m} / \mathrm{z}:[\mathrm{M}+\mathrm{Na}]^{+} \mathrm{C}_{31} \mathrm{H}_{33} \mathrm{NNaO}_{4}$ Calcd. 506.2307, Found 506.2309. 


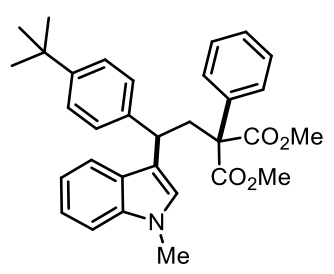

Following the general procedure, D-A cyclopropane $(1 \mathrm{e}, 0.029 \mathrm{~g}, 0.1 \mathrm{mmol})$ was subjected to 1,3-bisarylation using 1-methyl indole and $\mathrm{Ph}_{3} \mathrm{BiCl}_{2}$ as arene sources and the crude product was purified via silica gel column chromatography (EtOAc: Hexane 2:8), which afforded compound $4 \mathrm{e}$ in $87 \%$ yield $(0.043 \mathrm{~g})$ as colourless oil. $R_{f} 0.35$ (EtOAc: Hexane 3:7); ${ }^{1} \mathrm{H}$ NMR $\left(\mathrm{CDCl}_{3}, 400 \mathrm{MHz}\right) \delta 7.62-7.60(\mathrm{~m}, 2 \mathrm{H}), 7.43$ (d, $J=7.9 \mathrm{~Hz}, 1 \mathrm{H}), 7.39-7.32(\mathrm{~m}, 3 \mathrm{H}), 7.28-7.24(\mathrm{~m}, 5 \mathrm{H}), 7.19(\mathrm{t}, J=7.5 \mathrm{~Hz}, 1 \mathrm{H})$, $7.01(\mathrm{t}, J=7.4 \mathrm{~Hz}, 1 \mathrm{H}), 6.86(\mathrm{~s}, 1 \mathrm{H}), 4.30(\mathrm{t}, J=6.4 \mathrm{~Hz}, 1 \mathrm{H}), 3.73(\mathrm{~s}, 3 \mathrm{H}), 3.37(\mathrm{~s}$, $3 \mathrm{H}), 3.35-3.22(\mathrm{~m}, 5 \mathrm{H}), 1.30(\mathrm{~s}, 9 \mathrm{H}) ;{ }^{13} \mathrm{C}\left\{{ }^{1} \mathrm{H}\right\} \mathrm{NMR}\left(\mathrm{CDCl}_{3}, 100 \mathrm{MHz}\right) \delta 171.0$, $170.7,148.7,141.5,137.2$ (2), 128.5, 128.3, 127.9, 127.7, 127.0, 126.5, 125.1, 121.6, 119.8, 118.8, 118.7, 109.1, 61.6, 52.4, 52.2, 42.1, 38.1, 34.4, 32.8, 31.5; HRMS (ESI-TOF) m/z: $[\mathrm{M}+\mathrm{Na}]^{+} \mathrm{C}_{32} \mathrm{H}_{35} \mathrm{NNaO}_{4}$ Calcd. 520.2464, Found 520.2460.

\section{Diethyl}

2-(2-(4-methoxyphenyl)-2-(1-methyl-1H-indol-3-yl)ethyl)-2phenylmalonate (4f):<smiles>CCOC(CC)(CC(c1ccccc1)c1ccc(OC)cc1)c1ccccc1</smiles>

Following the general procedure, D-A cyclopropane $(1 \mathbf{f}, 0.029 \mathrm{~g}, 0.1 \mathrm{mmol})$ was subjected to 1,3-bisarylation using 1-methyl indole and $\mathrm{Ph}_{3} \mathrm{BiCl}_{2}$ as arene sources and the crude product was purified via silica gel column chromatography (EtOAc: Hexane 2:8), which afforded compound $\mathbf{4 f}$ in $88 \%$ yield $(0.044 \mathrm{~g})$ as colourless oil. $R_{f}$ 0.35 (EtOAc: Hexane 3:7); FT-IR $\left(\mathrm{v} \mathrm{cm}^{-1}\right): 2928,1730,1610,1510,1227,1177 ;{ }^{1} \mathbf{H}$ NMR $\left(\mathrm{CDCl}_{3}, 400 \mathrm{MHz}\right) \delta 7.61(\mathrm{~d}, J=7.3 \mathrm{~Hz}, 2 \mathrm{H}), 7.34-7.26(\mathrm{~m}, 4 \mathrm{H}), 7.23-7.19$ $(\mathrm{m}, 3 \mathrm{H}), 7.13(\mathrm{t}, J=7.5 \mathrm{~Hz}, 1 \mathrm{H}), 6.97(\mathrm{t}, J=7.4 \mathrm{~Hz}, 1 \mathrm{H}), 6.78-6.75(\mathrm{~m}, 3 \mathrm{H}), 4.24(\mathrm{t}$, $J=6.4 \mathrm{~Hz}, 1 \mathrm{H}), 3.88-3.76(\mathrm{~m}, 3 \mathrm{H}), 3.73(\mathrm{~s}, 3 \mathrm{H}), 3.67(\mathrm{~s}, 3 \mathrm{H}), 3.60-3.54(\mathrm{~m}, 1 \mathrm{H})$, $3.28-3.15(\mathrm{~m}, 2 \mathrm{H}), 1.04(\mathrm{t}, J=7.1 \mathrm{~Hz}, 6 \mathrm{H}) ;{ }^{13} \mathrm{C}\left\{{ }^{1} \mathrm{H}\right\} \mathrm{NMR}\left(\mathrm{CDCl}_{3}, 100 \mathrm{MHz}\right) \delta$ $170.6,170.2,157.9,137.3$ (2), 136.9, 129.3, 128.5, 128.1, 127.5, 127.0, 126.4, 121.5, 119.8, 119.0, 118.8, 113.6, 109.1, 61.6, 61.3, 61.2, 55.3, 42.1, 37.8, 32.7, 13.8, 13.7; HRMS (ESI-TOF) m/z: [M+Na] ${ }^{+} \mathrm{C}_{31} \mathrm{H}_{33} \mathrm{NNaO}_{5}$ Calcd. 522.2256, Found 522.2247 . 
<smiles>COc1ccc(C(CC(C)(O)c2ccccc2)c2cn(C)c3ccccc23)cc1</smiles>

Following the general procedure, D-A cyclopropane $(1 \mathrm{~g}, 0.029 \mathrm{~g}, 0.1 \mathrm{mmol})$ was subjected to 1,3-bisarylation using 1-methyl indole and $\mathrm{Ph}_{3} \mathrm{BiCl}_{2}$ as arene sources and the crude product was purified via silica gel column chromatography (EtOAc: Hexane 2:8), which afforded compound $\mathbf{4 g}$ in $83 \%$ yield $(0.046 \mathrm{~g})$ as colourless oil. $R_{f} 0.35$ (EtOAc: Hexane 3:7); ${ }^{1} \mathrm{H}$ NMR $\left(\mathrm{CDCl}_{3}, 400 \mathrm{MHz}\right) \delta 7.45-7.43(\mathrm{~m}, 2 \mathrm{H}), 7.24$ $(\mathrm{d}, J=3.3 \mathrm{~Hz}, 1 \mathrm{H}), 7.16-7.12(\mathrm{~m}, 5 \mathrm{H}), 7.10-7.06(\mathrm{~m}, 2 \mathrm{H}), 6.91(\mathrm{t}, J=7.4 \mathrm{~Hz}, 1 \mathrm{H})$, $6.72(\mathrm{~s}, 1 \mathrm{H}), 6.66(\mathrm{~d}, J=8.4 \mathrm{~Hz}, 2 \mathrm{H}), 4.13(\mathrm{t}, J=6.1 \mathrm{~Hz}, 1 \mathrm{H}), 3.68(\mathrm{~s}, 3 \mathrm{H}), 3.61(\mathrm{~s}$, $3 \mathrm{H}$ ), 3.16 (dd, $J=14.6,6.0 \mathrm{~Hz}, 1 \mathrm{H}), 3.07$ (dd, $J=14.6,6.2 \mathrm{~Hz}, 1 \mathrm{H}), 1.24(\mathrm{~s}, 9 \mathrm{H})$, $1.23(\mathrm{~s}, 9 \mathrm{H}) ;{ }^{13} \mathrm{C}\left\{{ }^{1} \mathrm{H}\right\}$ NMR $\left(\mathrm{CDCl}_{3}, 100 \mathrm{MHz}\right) \delta 169.9,169.7,157.7,138.1,137.7$, 137.3, 129.1, 128.8, 127.6, 127.1, 126.8, 126.3, 121.4, 119.9, 119.4, 118.6, 113.7, 109.0, 81.73, 81.7, 63.3, 55.4, 42.0, 37.8, 32.6, 27.7 (2); HRMS (ESI-TOF) m/z: $[\mathrm{M}+\mathrm{Na}]^{+} \mathrm{C}_{35} \mathrm{H}_{41} \mathrm{NNaO}_{5}$ Calcd. 578.2882, Found 578.2871.

Dimethyl phenylmalonate (4h):

2-(2-(3-methoxyphenyl)-2-(1-methyl-1H-indol-3-yl)ethyl)-2-<smiles></smiles>

Following the general procedure, D-A cyclopropane $(1 \mathrm{~h}, 0.027 \mathrm{~g}, 0.1 \mathrm{mmol})$ was subjected to 1,3-bisarylation using 1-methyl indole and $\mathrm{Ph}_{3} \mathrm{BiCl}_{2}$ as arene sources and the crude product was purified via silica gel column chromatography (EtOAc: Hexane 2:8), which afforded compound $\mathbf{4 h}$ in $83 \%$ yield $(0.039 \mathrm{~g})$ as colourless oil. $R_{f} 0.35$ (EtOAc: Hexane 3:7); ${ }^{1} \mathrm{H}$ NMR $\left(\mathrm{CDCl}_{3}, 400 \mathrm{MHz}\right) \delta 7.57-7.55(\mathrm{~m}, 2 \mathrm{H}), 7.38$ $(\mathrm{d}, J=8.0 \mathrm{~Hz}, 1 \mathrm{H}), 7.35-7.29(\mathrm{~m}, 3 \mathrm{H}), 7.21(\mathrm{~d}, J=8.2 \mathrm{~Hz}, 1 \mathrm{H}), 7.17-7.13(\mathrm{~m}, 2 \mathrm{H})$, 6.99 (t, J = 7.4 Hz, 1H), $6.90(\mathrm{~d}, J=7.7 \mathrm{~Hz}, 1 \mathrm{H}), 6.84(\mathrm{~s}, 1 \mathrm{H}), 6.81(\mathrm{~s}, 1 \mathrm{H}), 6.66$ (dd, $J=8.0,2.5 \mathrm{~Hz}, 1 \mathrm{H}), 4.25(\mathrm{t}, J=6.4 \mathrm{~Hz}, 1 \mathrm{H}), 3.75(\mathrm{~s}, 3 \mathrm{H}), 3.70(\mathrm{~s}, 3 \mathrm{H}), 3.35(\mathrm{~s}, 3 \mathrm{H})$, $3.30(\mathrm{~s}, 3 \mathrm{H}), 3.28-3.16(\mathrm{~m}, 2 \mathrm{H}) ;{ }^{13} \mathrm{C}\left\{{ }^{1} \mathrm{H}\right\}$ NMR $\left(\mathrm{CDCl}_{3}, 100 \mathrm{MHz}\right) \delta 171.0,170.7$, 159.6, 146.4, 137.2, 137.1, 129.2, 128.4, 128.3, 127.7, 127.0, 126.6, 121.6, 120.8, 119.8, 118.9, 118.3, 114.3, 111.3, 109.2, 61.7, 55.3, 52.4, 52.3, 42.0, 38.7, 32.8; HRMS (ESI-TOF) m/z: [M+Na] ${ }^{+} \mathrm{C}_{29} \mathrm{H}_{29} \mathrm{NNaO}_{5}$ Calcd. 494.1943, Found 494.1937. 
<smiles>COc1ccc(C(CC(C)(OC)c2ccccc2)c2ccccc2[N+](=O)[O-])cc1</smiles>

Following the general procedure, D-A cyclopropane $(1 \mathrm{i}, 0.030 \mathrm{~g}, 0.1 \mathrm{mmol})$ was subjected to 1,3-bisarylation using 1-methyl indole and $\mathrm{Ph}_{3} \mathrm{BiCl}_{2}$ as arene sources and the crude product was purified via silica gel column chromatography (EtOAc: Hexane 2:8), which afforded compound $4 \mathbf{i}$ in $86 \%$ yield $(0.043 \mathrm{~g})$ as colorless oil. $R_{f}$ 0.20 (EtOAc: Hexane 3:7); ${ }^{1} \mathrm{H}$ NMR $\left(\mathrm{CDCl}_{3}, 400 \mathrm{MHz}\right) \delta 7.54(\mathrm{~d}, \mathrm{~J}=8.0 \mathrm{~Hz}, 2 \mathrm{H})$, $7.36(\mathrm{~d}, J=8.0 \mathrm{~Hz}, 1 \mathrm{H}), 7.32-7.24(\mathrm{~m}, 3 \mathrm{H}), 7.19(\mathrm{~d}, J=8.2 \mathrm{~Hz}, 1 \mathrm{H}), 7.12(\mathrm{t}, J=7.5$ $\mathrm{Hz}, 1 \mathrm{H}), 6.96(\mathrm{t}, J=7.4 \mathrm{~Hz}, 1 \mathrm{H}), 6.83-6.78(\mathrm{~m}, 2 \mathrm{H}), 6.75(\mathrm{~s}, 1 \mathrm{H}), 6.70(\mathrm{~d}, J=8.2$ $\mathrm{Hz}, 1 \mathrm{H}), 4.20(\mathrm{t}, J=6.3 \mathrm{~Hz}, 1 \mathrm{H}), 3.78(\mathrm{~d}, J=2.6 \mathrm{~Hz}, 6 \mathrm{H}), 3.66(\mathrm{~s}, 3 \mathrm{H}), 3.33(\mathrm{~s}, 3 \mathrm{H})$, 3.29 (s, 3H), 3.24 (dd, J = 14.5, $5.5 \mathrm{~Hz}, 1 \mathrm{H}), 3.14(\mathrm{dd}, J=14.4,7.3 \mathrm{~Hz}, 1 \mathrm{H}) ;{ }^{13} \mathrm{C}\left\{{ }^{1} \mathrm{H}\right\}$ NMR $\left(\mathrm{CDCl}_{3}, 100 \mathrm{MHz}\right) \delta 171.0,170.7,148.7,147.3,137.3,137.2,128.4,128.3$, 127.7, 126.9, 126.4, 121.6, 120.2, 119.8, 118.9, 118.7, 111.9, 111.0, 109.2, 61.6, $56.0, \quad 56.0, \quad 52.4, \quad 52.3, \quad 42.1, \quad 38.3, \quad 32.7$; HRMS (ESI-TOF) $\mathrm{m} / \mathrm{z}: \quad[\mathrm{M}+\mathrm{Na}]^{+}$ $\mathrm{C}_{30} \mathrm{H}_{31} \mathrm{NNaO}_{6}$ Calcd. 524.2049, Found 524.2022.

Dimethyl 2-(2-(1-methyl-1H-indol-3-yl)-2-(3,4,5-trimethoxyphenyl)ethyl)-2phenylmalonate $(4 \mathrm{j})$ :<smiles>COc1cc(C(CC(C)(O[Na])c2ccccc2)c2cn(C)c3ccccc23)cc(OC)c1OC</smiles>

Following the general procedure, D-A cyclopropane $(1 \mathbf{j}, 0.032 \mathrm{~g}, 0.1 \mathrm{mmol})$ was subjected to 1,3-bisarylation using 1-methyl indole and $\mathrm{Ph}_{3} \mathrm{BiCl}_{2}$ as arene sources and the crude product was purified via silica gel column chromatography (EtOAc: Hexane $3: 7)$, which afforded compound $4 \mathrm{j}$ in $82 \%$ yield $(0.044 \mathrm{~g})$ as colourless oil. $R_{f}$ 0.20 (EtOAc: Hexane 3:7); FT-IR ( $\left.\mathrm{v} \mathrm{cm}^{-1}\right)$ : 2950, 1734, 1590, 1461, 1233, 1126; ${ }^{1} \mathbf{H}$ NMR $\left(\mathrm{CDCl}_{3}, 400 \mathrm{MHz}\right)$ ठ 7.57 - $7.54(\mathrm{~m}, 2 \mathrm{H}), 7.44(\mathrm{~d}, \mathrm{~J}=8.0 \mathrm{~Hz}, 1 \mathrm{H}), 7.34-7.28(\mathrm{~m}$, $3 \mathrm{H}), 7.24(\mathrm{~d}, J=8.2 \mathrm{~Hz}, 1 \mathrm{H}), 7.17(\mathrm{t}, J=8.0 \mathrm{~Hz}, 1 \mathrm{H}), 7.02(\mathrm{t}, J=8.0 \mathrm{~Hz}, 1 \mathrm{H}), 6.82$ (s, 1H), $6.49(\mathrm{~s}, 2 \mathrm{H}), 4.22(\mathrm{t}, J=6.3 \mathrm{~Hz}, 1 \mathrm{H}), 3.79(\mathrm{~s}, 6 \mathrm{H}), 3.77(\mathrm{~s}, 3 \mathrm{H}), 3.72(\mathrm{~s}, 3 \mathrm{H})$, 3.38 (s, 3H), 3.36 (s, 3H), 3.28 (dd, J = 14.5, $5.5 \mathrm{~Hz}, 1 \mathrm{H}$ ), 3.14 (dd, J = 14.5, $7.1 \mathrm{~Hz}$, $1 \mathrm{H}) ;{ }^{13} \mathbf{C}\left\{{ }^{1} \mathrm{H}\right\}$ NMR $\left(\mathrm{CDCl}_{3}, 100 \mathrm{MHz}\right) \delta 171.0,170.8,152.9,140.5,137.3,137.0$, 136.3, 128.4, 128.3, 127.8, 127.0, 126.5, 121.7, 119.8, 119.0, 118.3, 109.3, 105.5, $61.7,60.9,56.2,52.4,42.1,39.2$, 32.8; HRMS (ESI, m/z): $[\mathrm{M}+\mathrm{Na}]^{+} \mathrm{C}_{31} \mathrm{H}_{33} \mathrm{NNaO}_{7}$ Calcd. 554.2155, Found 554.2152. 
<smiles>COC(=O)c1ccccc1CC(CC(c1ccccc1)c1ccccc1)(OC)c1ccccc1</smiles>

Following the general procedure, D-A cyclopropane $(1 \mathrm{k}, 0.025 \mathrm{~g}, 0.1 \mathrm{mmol})$ was subjected to 1,3-bisarylation using 1-methyl indole and $\mathrm{Ph}_{3} \mathrm{BiCl}_{2}$ as arene sources and the crude product was purified via silica gel column chromatography (EtOAc: Hexane 2:8), which afforded compound $4 \mathbf{k}$ in $81 \%$ yield $(0.037 \mathrm{~g})$ as brown solid. mp: $125-128^{\circ} \mathrm{C} . R_{f} 0.35$ (EtOAc: Hexane 3:7); ${ }^{1} \mathrm{H}$ NMR $\left(\mathrm{CDCl}_{3}, 400 \mathrm{MHz}\right) \delta 7.62$ $7.60(\mathrm{~m}, 2 \mathrm{H}), 7.50(\mathrm{~d}, J=7.7 \mathrm{~Hz}, 1 \mathrm{H}), 7.45(\mathrm{~d}, J=8.0 \mathrm{~Hz}, 1 \mathrm{H}), 7.37-7.30(\mathrm{~m}, 3 \mathrm{H})$, 7.23-7.13 (m, 3H), 7.07-7.02 (m, 3H), $6.57(\mathrm{~s}, 1 \mathrm{H}), 4.59(\mathrm{dd}, J=8.3,4.6 \mathrm{~Hz}, 1 \mathrm{H})$, $3.66(\mathrm{~s}, 3 \mathrm{H}), 3.39(\mathrm{~s}, 3 \mathrm{H}), 3.32-3.27(\mathrm{~m}, 4 \mathrm{H}), 3.16(\mathrm{dd}, J=14.6,8.4 \mathrm{~Hz}, 1 \mathrm{H}), 2.16(\mathrm{~s}$, $3 \mathrm{H}) ;{ }^{13} \mathrm{C}\left\{{ }^{1} \mathrm{H}\right\}$ NMR $\left(\mathrm{CDCl}_{3}, 100 \mathrm{MHz}\right) \delta 171.0,170.8,141.9,137.5,137.2,136.5$, 130.6, 128.3, 127.7, 127.4, 127.0, 126.0, 125.8, 121.6, 119.7, 118.9, 118.5, 109.2, 61.5, 52.4, 52.3, 42.2, 33.7, 32.7, 19.8; HRMS (ESI-TOF) m/z: [M+Na] $\mathrm{C}_{29} \mathrm{H}_{29} \mathrm{NNaO}_{4}$ Calcd. 478.1994, Found 478.1974.

Dimethyl

2-(2-(2-methoxyphenyl)-2-(1-methyl-1H-indol-3-yl)ethyl)-2phenylmalonate (4I):<smiles>COC(Cc1ccccc1)(CC(c1ccccc1)c1ccccc1)c1ccccc1</smiles>

Following the general procedure, D-A cyclopropane $(1 \mathrm{I}, 0.027 \mathrm{~g}, 0.1 \mathrm{mmol}$ ) was subjected to 1,3-bisarylation using 1-methyl indole and $\mathrm{Ph}_{3} \mathrm{BiCl}_{2}$ as arene sources and the crude product was purified via silica gel column chromatography (EtOAc: Hexane 2:8), which afforded compound $4 \mathrm{I}$ in $72 \%$ yield $(0.034 \mathrm{~g})$ as colourless oil. $R_{f}$ 0.30 (EtOAc: Hexane 3:7); FT-IR $\left(\mathrm{v} \mathrm{cm}^{-1}\right): 2951,1735,1598,1490,1233 ;{ }^{1} \mathbf{H}$ NMR $\left(\mathrm{CDCl}_{3}, 400 \mathrm{MHz}\right) \delta 7.61-7.58(\mathrm{~m}, 2 \mathrm{H}), 7.40(\mathrm{~d}, J=8.0 \mathrm{~Hz}, 1 \mathrm{H}), 7.35-7.29(\mathrm{~m}, 4 \mathrm{H})$, $7.19(\mathrm{~d}, J=8.2 \mathrm{~Hz}, 1 \mathrm{H}), 7.15-7.07(\mathrm{~m}, 2 \mathrm{H}), 6.98(\mathrm{t}, J=7.5 \mathrm{~Hz}, 1 \mathrm{H}), 6.84(\mathrm{t}, J=7.5$ $\mathrm{Hz}, 1 \mathrm{H}), 6.81(\mathrm{~s}, 1 \mathrm{H}), 6.78(\mathrm{~d}, J=8.2 \mathrm{~Hz}, 1 \mathrm{H}), 4.76(\mathrm{t}, J=6.6 \mathrm{~Hz}, 1 \mathrm{H}), 3.74(\mathrm{~s}, 3 \mathrm{H})$, $3.68(\mathrm{~s}, 3 \mathrm{H}), 3.33(\mathrm{~s}, 3 \mathrm{H}), 3.31(\mathrm{~s}, 3 \mathrm{H}), 3.27-3.17(\mathrm{~m}, 2 \mathrm{H}) ;{ }^{13} \mathrm{C}\left\{{ }^{1} \mathrm{H}\right\}$ NMR $\left(\mathrm{CDCl}_{3}, 100 \mathrm{MHz}\right) \delta 171.1,170.7,156.9,137.2,137.1,132.7,129.1,128.6,128.1$, $127.5,127.4,127.1,126.9,121.4,120.4,112.0,118.7,118.4,110.9,109.0,61.7$, 55.4, 52.3, 52.3, 41.6, 32.8, 30.8; HRMS (ESI-TOF) m/z: $[\mathrm{M}+\mathrm{Na}]^{+} \mathrm{C}_{29} \mathrm{H}_{29} \mathrm{NNaO}_{5}$ Calcd. 494.1943, Found 494.1930. 
<smiles>COC(CC(c1ccccc1)c1cn(C)c2ccccc12)(OC)c1ccc(F)cc1</smiles>

Following the general procedure, D-A cyclopropane $(1 \mathrm{~m}, 0.025 \mathrm{~g}, 0.1 \mathrm{mmol})$ was subjected to 1,3-bisarylation using 1-methyl indole and $\mathrm{Ph}_{3} \mathrm{BiCl}_{2}$ as arene sources and the crude product was purified via silica gel column chromatography (EtOAc: Hexane 2:8), which afforded compound $4 \mathrm{~m}$ in $59 \%$ yield $(0.027 \mathrm{~g})$ as colourless oil. $R_{f} 0.35$ (EtOAc: Hexane 3:7); FT-IR $\left(\mathrm{v} \mathrm{cm}^{-1}\right): 2951,1733,1602,1507,1228 ;{ }^{1} \mathbf{H}$ NMR $\left(\mathrm{CDCl}_{3}, 400 \mathrm{MHz}\right) \delta 7.53(\mathrm{~d}, J=7.4 \mathrm{~Hz}, 2 \mathrm{H}), 7.30-7.28(\mathrm{~m}, 4 \mathrm{H}), 7.24-7.18(\mathrm{~m}, 3 \mathrm{H})$, $7.12(\mathrm{t}, J=7.6 \mathrm{~Hz}, 1 \mathrm{H}), 6.96(\mathrm{t}, J=7.5 \mathrm{~Hz}, 1 \mathrm{H}), 6.88(\operatorname{app~t}, J=8.6 \mathrm{~Hz}, 2 \mathrm{H}), 6.76(\mathrm{~s}$, $1 \mathrm{H}), 4.27(\mathrm{t}, J=6.3 \mathrm{~Hz}, 1 \mathrm{H}), 3.66(\mathrm{~s}, 3 \mathrm{H}), 3.35(\mathrm{~s}, 3 \mathrm{H}), 3.27-3.23(\mathrm{~m}, 4 \mathrm{H}), 3.14(\mathrm{dd}, J$ $=14.5,7.2 \mathrm{~Hz}, 1 \mathrm{H}) ;{ }^{13} \mathrm{C}\left\{{ }^{1} \mathrm{H}\right\}$ NMR $\left(\mathrm{CDCl}_{3}, 100 \mathrm{MHz}\right) \delta 170.9,170.6,161.3(\mathrm{~d} \mathrm{~J}=$ $242.5 \mathrm{~Hz}), 140.4(\mathrm{~d} \mathrm{~J}=3.1 \mathrm{~Hz}), 137.2,137.0,129.8(\mathrm{~d}, \mathrm{~J}=7.7 \mathrm{~Hz}), 128.3(2), 127.8$, 126.8, 126.4, 121.7, 119.6, 119.0, 118.3, 114.9(d = 20.8 Hz), 109.2, 61.5, 52.5, 52.3, 42.1, 38.0, 32.7; HRMS (ESI-TOF) $\mathrm{m} / \mathrm{z}$ : $[\mathrm{M}+\mathrm{Na}]^{+} \mathrm{C}_{28} \mathrm{H}_{26} \mathrm{FNNaO}_{4}$ Calcd. 482.1744, Found 482.1737.

\section{Dimethyl}

2-(2-(4-chlorophenyl)-2-(1-methyl-1H-indol-3-yl)ethyl)-2phenylmalonate (4n):<smiles>COC(CC(c1ccccc1)c1ccn(C)c1)(OC)c1ccccc1</smiles>

Following the general procedure, D-A cyclopropane $(1 \mathrm{n}, 0.027 \mathrm{~g}, 0.1 \mathrm{mmol})$ was subjected to 1,3-bisarylation using 1-methyl indole and $\mathrm{Ph}_{3} \mathrm{BiCl}_{2}$ as arene sources and the crude product was purified via silica gel column chromatography (EtOAc: Hexane 2:8), which afforded compound $\mathbf{4 n}$ in $55 \%$ yield $(0.026 \mathrm{~g})$ as colourless oil. $R_{f} 0.35$ (EtOAc: Hexane 3:7); ${ }^{1} \mathrm{H}$ NMR $\left(\mathrm{CDCl}_{3}, 400 \mathrm{MHz}\right) \delta 7.55-7.53(\mathrm{~m}, 2 \mathrm{H}), 7.34-$ $7.29(\mathrm{~m}, 4 \mathrm{H}), 7.24-7.14(\mathrm{~m}, 6 \mathrm{H}), 6.99(\mathrm{t}, J=7.5,1 \mathrm{H}), 6.79(\mathrm{~s}, 1 \mathrm{H}), 4.28(\mathrm{t}, J=6.4$ $\mathrm{Hz}, 1 \mathrm{H}), 3.71(\mathrm{~s}, 3 \mathrm{H}), 3.38(\mathrm{~s}, 3 \mathrm{H}), 3.30-3.12(\mathrm{~m}, 4 \mathrm{H}), 3.15(\mathrm{dd}, J=14.5,7.2 \mathrm{~Hz}$, $1 \mathrm{H}) ;{ }^{13} \mathrm{C}\left\{{ }^{1} \mathrm{H}\right\}$ NMR $\left(\mathrm{CDCl}_{3}, 100 \mathrm{MHz}\right) \delta 171.0,170.7,143.2,137.3,137.0,131.8$, 129.7, 128.4 (2), 128.3, 127.8, 126.8, 126.5, 121.8, 119.6, 119.0, 118.0, 109.3, 61.6, 52.5, 52.4, 41.9, 38.2, 32.8; HRMS (ESI-TOF) m/z: [M+Na] ${ }^{+} \mathrm{C}_{28} \mathrm{H}_{26} \mathrm{CINNaO}_{4}$ Calcd. 498.1448, Found 498.1443 . 
Dimethyl phenylmalonate (40):<smiles>COC(=O)C(CC(c1ccccc1)c1cn(C)c2ccccc12)(OC)c1ccc(Br)cc1</smiles>

Following the general procedure, D-A cyclopropane $(10,0.031 \mathrm{~g}, 0.1 \mathrm{mmol})$ was subjected to 1,3-bisarylation using 1-methyl indole and $\mathrm{Ph}_{3} \mathrm{BiCl}_{2}$ as arene sources and the crude product was purified via silica gel column chromatography (EtOAc: Hexane 2:8), which afforded compound 40 in $58 \%$ yield $(0.030 \mathrm{~g})$ as colourless oil. $R_{f} 0.35$ (EtOAc: Hexane 3:7); ${ }^{1} \mathrm{H}$ NMR $\left(\mathrm{CDCl}_{3}, 400 \mathrm{MHz}\right) \delta 7.54(\mathrm{~d}, J=7.0 \mathrm{~Hz}, 2 \mathrm{H})$, $7.35-7.28(\mathrm{~m}, 6 \mathrm{H}), 7.23(\mathrm{~d}, J=8.2 \mathrm{~Hz}, 1 \mathrm{H}), 7.18-7.14(\mathrm{~m}, 3 \mathrm{H}), 7.00(\mathrm{t}, J=7.4 \mathrm{~Hz}$, $1 \mathrm{H}), 6.80(\mathrm{~s}, 1 \mathrm{H}), 4.27(\mathrm{t}, J=6.3 \mathrm{~Hz}, 1 \mathrm{H}), 3.71(\mathrm{~s}, 3 \mathrm{H}), 3.39(\mathrm{~s}, 3 \mathrm{H}), 3.30-3.25(\mathrm{~m}$, $4 \mathrm{H}), 3.15(\mathrm{dd}, J=14.5,7.1 \mathrm{~Hz}, 1 \mathrm{H}) ;{ }^{13} \mathrm{C}\left\{{ }^{1} \mathrm{H}\right\}$ NMR $\left(\mathrm{CDCl}_{3}, 100 \mathrm{MHz}\right) \delta 170.9,170.7$, 143.8, 137.3, 137.0, 131.3, 130.1, 128.4, 128.3, 127.1, 126.8 126.5, 121.8, 119.9, 119.6, 119.0, 117.9, 109.3, 61.6, 52.5, 52.4, 41.8, 38.2, 32.8; HRMS (ESI-TOF) m/z: $[\mathrm{M}+\mathrm{H}]^{+} \mathrm{C}_{28} \mathrm{H}_{27} \mathrm{BrNO}_{4}$ Calcd. 520.1123, Found 520.1112.

Dimethyl phenylmalonate $(4 p)$ :<smiles></smiles>

Following the general procedure, D-A cyclopropane $(1 \mathrm{p}, 0.031 \mathrm{~g}, 0.1 \mathrm{mmol})$ was subjected to 1,3-bisarylation using 1-methyl indole and $\mathrm{Ph}_{3} \mathrm{BiCl}_{2}$ as arene sources and the crude product was purified via silica gel column chromatography (EtOAc: Hexane 2:8), which afforded compound $4 \mathrm{p}$ in $54 \%$ yield $(0.028 \mathrm{~g})$ as colourless oil. $R_{f} 0.35$ (EtOAc: Hexane 3:7); ${ }^{1} \mathrm{H}$ NMR $\left(\mathrm{CDCl}_{3}, 400 \mathrm{MHz}\right) \delta 7.59-7.55(\mathrm{~m}, 3 \mathrm{H}), 7.52$ $(\mathrm{d}, J=7.8 \mathrm{~Hz}, 1 \mathrm{H}), 7.46(\mathrm{~d}, J=8.0 \mathrm{~Hz}, 1 \mathrm{H}), 7.33-7.28(\mathrm{~m}, 3 \mathrm{H}), 7.23-7.14(\mathrm{~m}, 3 \mathrm{H})$, $7.04(\mathrm{t}, J=7.3 \mathrm{~Hz}, 1 \mathrm{H}), 6.96(\mathrm{t}, J=7.6 \mathrm{~Hz}, 1 \mathrm{H}), 6.70(\mathrm{~s}, 1 \mathrm{H}), 4.90(\mathrm{t}, J=6.6 \mathrm{~Hz}, 1 \mathrm{H})$, $3.63(\mathrm{~s}, 3 \mathrm{H}), 3.44(\mathrm{~s}, 3 \mathrm{H}), 3.36-3.31(\mathrm{~m}, 4 \mathrm{H}), 3.11(\mathrm{dd}, J=14.6,7.2 \mathrm{~Hz}, 1 \mathrm{H}) ;{ }^{13} \mathrm{C}$ $\left\{{ }^{1} \mathrm{H}\right\}$ NMR $\left(\mathrm{CDCl}_{3}, 100 \mathrm{MHz}\right) \delta 170.7(2), 143.3,137.1,136.9,133.1,129.2,128.4$, 128.3, 127.6 (2), 127.3, 126.9, 124.9, 121.6, 112.0, 119.0, 117.1, 109.2, 96.2, 61.6, 52.5, 52.3, 42.0, 37.4, 32.7; HRMS (ESI-TOF) m/z: $[\mathrm{M}+\mathrm{H}]{ }^{+} \mathrm{C}_{28} \mathrm{H}_{26} \mathrm{BrNO}_{4}$ Calcd. 520.1123, Found 520.1114 . 


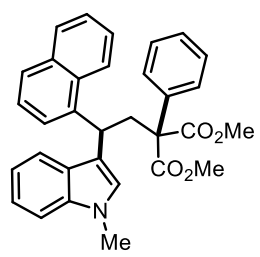

Following the general procedure, D-A cyclopropane $(1 \mathrm{q}, 0.028 \mathrm{~g}, 0.1 \mathrm{mmol})$ was subjected to 1,3-bisarylation using 1-methyl indole and $\mathrm{Ph}_{3} \mathrm{BiCl}_{2}$ as arene sources and the crude product was purified via silica gel column chromatography (EtOAc: Hexane 2:8), which afforded compound $\mathbf{4 q}$ in $81 \%$ yield $(0.040 \mathrm{~g})$ as colourless oil. $R_{f} 0.35$ (EtOAc: Hexane 3:7); ${ }^{1} \mathrm{H}$ NMR $\left(\mathrm{CDCl}_{3}, 400 \mathrm{MHz}\right) \delta 7.83-7.79(\mathrm{~m}, 2 \mathrm{H}), 7.69$ $(\mathrm{d}, J=8.2 \mathrm{~Hz}, 1 \mathrm{H}), 7.64-7.57(\mathrm{~m}, 4 \mathrm{H}), 7.44-7.39(\mathrm{~m}, 2 \mathrm{H}), 7.37-7.32(\mathrm{~m}, 4 \mathrm{H}), 7.23-$ $7.16(\mathrm{~m}, 2 \mathrm{H}), 7.05(\mathrm{t}, J=7.3 \mathrm{~Hz}, 1 \mathrm{H}), 6.68(\mathrm{~s}, 1 \mathrm{H}), 5.22(\mathrm{t}, J=6.6 \mathrm{~Hz}, 1 \mathrm{H}), 3.65(\mathrm{~s}$, $3 \mathrm{H}$ ), 3.45 (dd, $J=14.7,6.0 \mathrm{~Hz}, 1 \mathrm{H}), 3.40(\mathrm{~s}, 3 \mathrm{H}), 3.25(\mathrm{dd}, J=14.8,7.2 \mathrm{~Hz}, 1 \mathrm{H})$, $3.13(\mathrm{~s}, 3 \mathrm{H}) ;{ }^{13} \mathrm{C}\left\{{ }^{1} \mathrm{H}\right\}$ NMR $\left(\mathrm{CDCl}_{3}, 100 \mathrm{MHz}\right) \delta 171.0,170.8,139.9,137.5,137.3$, $134.2,131.6,128.8,128.5,128.4,127.7$ (2), 127.2, 127.0, 125.9, 125.4, 125.3, 124.8, 123.8, 121.6, 119.9, 119.0, 118.0, 109.2, 61.8, 52.5, 52.1, 42.5, 33.6, 32.8; HRMS (ESI-TOF) m/z: [M+Na] ${ }^{+} \mathrm{C}_{32} \mathrm{H}_{29} \mathrm{NNaO}_{4}$ Calcd. 514.1994, Found 514.1996.

\section{Dimethyl phenylmalonate (4r):}

\section{2-(2-(1-methyl-1H-indol-3-yl)-2-(naphthalen-2-yl)ethyl)-2-}<smiles>COC(C)(OC)c1ccccc1CC(c1ccc2ccccc2c1)c1cn([N+](=O)[O-])c2ccccc12</smiles>

Following the general procedure, D-A cyclopropane $(1 \mathbf{r}, 0.028 \mathrm{~g}, 0.1 \mathrm{mmol})$ was subjected to 1,3-bisarylation using 1-methyl indole and $\mathrm{Ph}_{3} \mathrm{BiCl}_{2}$ as arene sources and the crude product was purified via silica gel column chromatography (EtOAc: Hexane 2:8), which afforded compound $4 \mathrm{r}$ in $72 \%$ yield $(0.035 \mathrm{~g})$ as gummy solid. $R_{f}$ 0.25 (EtOAc: Hexane 3:7); ${ }^{1} \mathrm{H}$ NMR $\left(\mathrm{CDCl}_{3}, 400 \mathrm{MHz}\right) \delta 7.79-7.70(\mathrm{~m}, 4 \mathrm{H}), 7.60(\mathrm{~d}$, $J=7.5 \mathrm{~Hz}, 2 \mathrm{H}), 7.46-7.39(\mathrm{~m}, 4 \mathrm{H}), 7.37-7.30(\mathrm{~m}, 3 \mathrm{H}), 7.22(\mathrm{~d}, J=8.2 \mathrm{~Hz}, 1 \mathrm{H})$, $7.15(\mathrm{t}, J=7.6 \mathrm{~Hz}, 1 \mathrm{H}), 6.98(\mathrm{t}, J=7.4 \mathrm{~Hz}, 1 \mathrm{H}), 6.85(\mathrm{~s}, 1 \mathrm{H}), 4.49(\mathrm{t}, J=6.3 \mathrm{~Hz}, 1 \mathrm{H})$, $3.71(\mathrm{~s}, 3 \mathrm{H}), 3.36(\mathrm{~m}, 2 \mathrm{H}), 3.32(\mathrm{~s}, 3 \mathrm{H}), 3.18(\mathrm{~s}, 3 \mathrm{H}) ;{ }^{13} \mathrm{C}\left\{{ }^{1} \mathrm{H}\right\}$ NMR $\left(\mathrm{CDCl}_{3}, 100 \mathrm{MHz}\right)$ $\delta$ 171.1, 170.7, 141.8, 137.2, 137.1, 133.5, 132.2, 128.4 (2), 127.9, 127.8 (2), 127.6, $127.2,126.9,126.6,126.3,125.9,125.4,121.7,119.7,118.9,118.4,109.2,61.6$, 52.4, 52.3, 41.6, 38.7, 32.8; HRMS (ESI-TOF) m/z: $[\mathrm{M}+\mathrm{Na}]^{+} \mathrm{C}_{32} \mathrm{H}_{29} \mathrm{NNaO}_{4}$ Calcd. 514.1994, Found 514.1997. 

(4s):<smiles>COC(C)(CC(CC(OC)(c1ccccc1)c1ccccc1)(c1ccccc1)c1ccco1)OC</smiles>

Following the general procedure, D-A cyclopropane $(1 \mathrm{~s}, 0.022 \mathrm{~g}, 0.1 \mathrm{mmol}$ ) was subjected to 1,3-bisarylation using 1-methyl indole and $\mathrm{Ph}_{3} \mathrm{BiCl}_{2}$ as arene sources and the crude product was purified via silica gel column chromatography (EtOAc: Hexane 2:8), which afforded compound $4 \mathrm{~s}$ in $79 \%$ yield $(0.034 \mathrm{~g})$ as colourless oil. $R_{f} 0.20$ (EtOAc: Hexane 3:7); ${ }^{1} \mathrm{H}$ NMR $\left(\mathrm{CDCl}_{3}, 400 \mathrm{MHz}\right) \delta 7.57(\mathrm{~m}, 2 \mathrm{H}), 7.45$ (d, J = $8.0 \mathrm{~Hz}, 1 \mathrm{H}), 7.36-7.29(\mathrm{~m}, 4 \mathrm{H}), 7.24(\mathrm{~d}, J=8.2 \mathrm{~Hz}, 1 \mathrm{H}), 7.17(\mathrm{t}, J=7.6 \mathrm{~Hz}, 1 \mathrm{H})$, $7.03(\mathrm{t}, J=7.4 \mathrm{~Hz}, 1 \mathrm{H}), 6.83(\mathrm{~s}, 1 \mathrm{H}), 6.23-6.22(\mathrm{~m}, 1 \mathrm{H}), 6.01(\mathrm{~d}, J=3.2 \mathrm{~Hz}, 1 \mathrm{H})$, $4.33(\mathrm{t}, J=6.5 \mathrm{~Hz}, 1 \mathrm{H}), 3.71(\mathrm{~s}, 3 \mathrm{H}), 3.50(\mathrm{~s}, 3 \mathrm{H}), 3.34-3.29(\mathrm{~m}, 4 \mathrm{H}), 3.15(\mathrm{dd}, J=$ $14.5,6.1 \mathrm{~Hz}, 1 \mathrm{H}) ;{ }^{13} \mathrm{C}\left\{{ }^{1} \mathrm{H}\right\}$ NMR $\left(\mathrm{CDCl}_{3}, 100 \mathrm{MHz}\right) \delta 170.8,170.7,157.1,141.2$, $137.2,136.7,128.4,128.4,127.7,127.3,126.6,121.6,119.9,119.0,115.3,110.2$, 109.3, 105.9, 61.4, 52.7, 52.3, 39.7, 32.8, 32.7; HRMS (ESI-TOF) m/z: [M+Na] ${ }^{+}$ $\mathrm{C}_{26} \mathrm{H}_{25} \mathrm{NNaO}_{5}$ Calcd. 454.1630, Found 454.1628.

Dimethyl phenylmalonate(4t):<smiles>CO[C@H](Cc1ccccc1)Cc1cn(C)c2ccccc12</smiles>

2-(2-(1-methyl-1H-indol-3-yl)-2-(thiophen-2-yl)ethyl)-2-

Following the general procedure, D-A cyclopropane (1t, $0.048 \mathrm{~g}, 0.2 \mathrm{mmol}$ ) was subjected to 1,3-bisarylation using 1-methyl indole and $\mathrm{Ph}_{3} \mathrm{BiCl}_{2}$ as arene sources and the crude product was purified via silica gel column chromatography (EtOAc: Hexane 2:8), which afforded compound $4 \mathrm{t}$ in $88 \%$ yield $(0.079 \mathrm{~g})$ as colourless oil. $R_{f}$ 0.35 (EtOAc: Hexane 3:7); ${ }^{1} \mathrm{H}$ NMR $\left(\mathrm{CDCl}_{3}, 400 \mathrm{MHz}\right) \delta 7.59$ (d, J = 4.2 Hz, 2H), $7.43(\mathrm{~d}, J=8.0 \mathrm{~Hz}, 1 \mathrm{H}), 7.37-7.31(\mathrm{~m}, 3 \mathrm{H}), 7.24(\mathrm{~d}, J=8.1 \mathrm{~Hz}, 1 \mathrm{H}), 7.17(\mathrm{t}, J=7.5$ $\mathrm{Hz}, 1 \mathrm{H}), 7.08(\mathrm{~d}, J=5.0 \mathrm{~Hz}, 1 \mathrm{H}), 7.02(\mathrm{t}, J=7.4 \mathrm{~Hz}, 1 \mathrm{H}), 6.89-6.85(\mathrm{~m}, 2 \mathrm{H}), 6.83(\mathrm{~s}$, $1 \mathrm{H}), 4.54(\mathrm{t}, J=6.4 \mathrm{~Hz}, 1 \mathrm{H}), 3.71(\mathrm{~s}, 3 \mathrm{H}), 3.39(\mathrm{~s}, 3 \mathrm{H}), 3.37-3.29(\mathrm{~m}, 2 \mathrm{H}), 3.27(\mathrm{~s}$, $3 \mathrm{H}) ;{ }^{13} \mathrm{C}\left\{{ }^{1} \mathrm{H}\right\}$ NMR $\left(\mathrm{CDCl}_{3}, 100 \mathrm{MHz}\right) \delta$ 170.8, 170.7, 149.8, 137.3, 136.8, 128.4, 128.3, 127.8, 127.1, 126.6, 126.4, 124.2, 123.6, 121.7, 120.0, 119.0, 117.21, 109.3, $61.5,52.5,52.2,42.9,34.2,32.8$; HRMS (ESI-TOF) m/z: $[\mathrm{M}+\mathrm{Na}]^{+} \mathrm{C}_{26} \mathrm{H}_{25} \mathrm{NNaO}_{4} \mathrm{~S}$ Calcd. 470.1402, Found 470.1400. 

phenylmalonate $(4 u)$ :

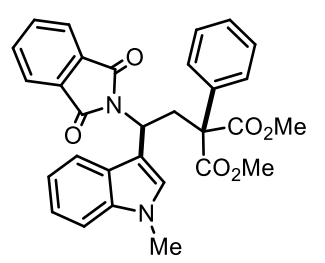

Following the general procedure, D-A cyclopropane $(1 \mathrm{u}, 0.030 \mathrm{~g}, 0.1 \mathrm{mmol})$ was subjected to 1,3-bisarylation using 1-methyl indole and $\mathrm{Ph}_{3} \mathrm{BiCl}_{2}$ as arene sources and the crude product was purified via silica gel column chromatography (EtOAc: Hexane 3:7), which afforded compound $4 \mathbf{u}$ in $82 \%$ yield $(0.042 \mathrm{~g})$ as colourless oil. $R_{f} 0.20$ (EtOAc: Hexane 3:7); FT-IR $\left(\mathrm{v} \mathrm{cm}^{-1}\right): 2953,1737,1709,1236 ;{ }^{1} \mathbf{H}$ NMR $\left(\mathrm{CDCl}_{3}, 400 \mathrm{MHz}\right) \delta 7.70(\mathrm{~d}, J=8.1 \mathrm{~Hz}, 1 \mathrm{H}), 7.66-7.63(\mathrm{~m},, 2 \mathrm{H}), 7.61-7.57(\mathrm{~m} \mathrm{2H})$, $7.44(\mathrm{~d}, J=7.6 \mathrm{~Hz}, 2 \mathrm{H}), 7.33(\mathrm{~s}, 1 \mathrm{H}), 7.23(\mathrm{~d}, J=8.1 \mathrm{~Hz}, 1 \mathrm{H}), 7.20-7.15(\mathrm{~m}, 3 \mathrm{H})$, $7.10-7.01(\mathrm{~m}, 2 \mathrm{H}), 5.97(\mathrm{dd}, J=9.1,3.9 \mathrm{~Hz}, 1 \mathrm{H}), 3.87(\mathrm{dd}, J=15.1,9.1 \mathrm{~Hz}, 1 \mathrm{H})$, $3.74(\mathrm{~s}, 3 \mathrm{H}), 3.67(\mathrm{~s}, 3 \mathrm{H}), 3.49(\mathrm{~s}, 3 \mathrm{H}), 3.30(\mathrm{dd}, J=15.0,3.9 \mathrm{~Hz}, 1 \mathrm{H}) ;{ }^{13} \mathrm{C}\left\{{ }^{1} \mathrm{H}\right\}$ NMR $\left(\mathrm{CDCl}_{3}, 100 \mathrm{MHz}\right) \delta 170.8,170.7,167.9,136.3,136.1,133.7,132.0,128.8,128.3$, 128.0, 127.5, 126.6, 122.9, 121.9, 119.6, 119.4, 113.7, 109.2, 61.6, 53.0, 52.9, 42.9, 38.2, 33.0; HRMS (ESI-TOF) m/z: [M+Na] ${ }^{+} \mathrm{C}_{30} \mathrm{H}_{26} \mathrm{~N}_{2} \mathrm{NaO}_{6}$ Calcd. 533.1689, Found 533.1684 .

Methyl 2-acetyl-4-(1-methyl-1H-indol-3-yl)-2,4-diphenylbutanoate (4v):

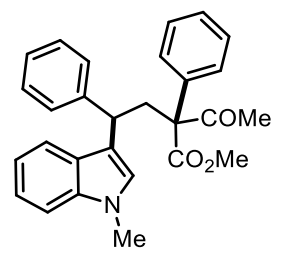

Following the general procedure, D-A cyclopropane $(1 \mathrm{v}, 0.022 \mathrm{~g}, 0.1 \mathrm{mmol})$ was subjected to 1,3-bisarylation using 1-methyl indole and $\mathrm{Ph}_{3} \mathrm{BiCl}_{2}$ as arene sources and the crude product was purified via silica gel column chromatography (EtOAc: Hexane 2:8), which afforded compound $\mathbf{4} \mathbf{v}$ in $54 \%$ yield $(0.023 \mathrm{~g})$ as an inseparable mixture of diastereomers ( $d r$ 10:1). $R_{f} 0.35$ (EtOAc: Hexane 3:7); ${ }^{1} \mathbf{H}$ NMR $\left(\mathrm{CDCl}_{3}\right.$, $400 \mathrm{MHz}) \delta 7.67(\mathrm{~d}, J=8.0 \mathrm{~Hz}, 1 \mathrm{H}), 7.49-7.47(\mathrm{~m}, 2 \mathrm{H}), 7.34-7.28(\mathrm{~m}, 3 \mathrm{H}), 7.25-$ $7.15(\mathrm{~m}, 6 \mathrm{H}), 7.10-7.03(\mathrm{~m}, 2 \mathrm{H}), 6.75(\mathrm{~s}, 1 \mathrm{H}), 4.22(\mathrm{t}, J=6.6 \mathrm{~Hz}, 1 \mathrm{H}), 3.72(\mathrm{~s}, 3 \mathrm{H})$, $3.40(\mathrm{dd}, J=14.5,6.8 \mathrm{~Hz}, 1 \mathrm{H}), 3.02(\mathrm{~s}, 3 \mathrm{H}), 2.95(\mathrm{dd}, J=14.5,6.4 \mathrm{~Hz}, 1 \mathrm{H}), 1.95(\mathrm{~s}$, $3 \mathrm{H}) ;{ }^{13} \mathrm{C}\left\{{ }^{1} \mathrm{H}\right\}$ NMR $\left(\mathrm{CDCl}_{3}, 100 \mathrm{MHz}\right) \delta 202.8,171.0,144.9,137.4,136.8,128.7$, 128.4, 128.2 (2), 127.8, 127.1, 127.0, 126.0, 121.6, 120.3, 119.0, 118.3, 109.2, 67.7, 51.6, 41.4, 39.0, 32.8, 27.4; HRMS (ESI, m/z): $[\mathrm{M}+\mathrm{Na}]^{+} \mathrm{C}_{28} \mathrm{H}_{27} \mathrm{NNaO}_{3}$ Calcd. 448.1889, Found 448.1883. 


\section{Preparation and characterization data of compounds $\mathbf{5}$}

Dimethyl 2-(2-(5-methoxy-1-methyl-1H-indol-3-yl)-2-(4-methoxyphenyl)ethyl)-2phenylmalonate (5a):<smiles>COCC(COC)(c1ccccc1)C(Cc1cn(C)c2ccc(OC)cc12)c1ccc(OC)cc1</smiles>

Following the general procedure, D-A cyclopropane $(1 \mathrm{a}, 0.027 \mathrm{~g}, 0.1 \mathrm{mmol})$ was subjected to 1,3-bisarylation using 6-methoxy-1-methyl indole and $\mathrm{Ph}_{3} \mathrm{BiCl}_{2}$ as arene sources and the crude product was purified via silica gel column chromatography (EtOAc: Hexane 2:8), which afforded compound $5 \mathrm{a}$ in $93 \%$ yield $(0.047 \mathrm{~g})$ as white solid. $\mathrm{mp}: 127-130{ }^{\circ} \mathrm{C}$; $R_{f} 0.25$ (EtOAc: Hexane 3:7); FT-IR $\left(\mathrm{v} \mathrm{cm}^{-1}\right): 2950,1734$, $1510,1492,1227 ;{ }^{1} \mathrm{H}$ NMR $\left(\mathrm{CDCl}_{3}, 400 \mathrm{MHz}\right) \delta 7.53(\mathrm{~d}, J=7.7 \mathrm{~Hz}, 2 \mathrm{H}), 7.31-7.22$ $(\mathrm{m}, 3 \mathrm{H}), 7.19-7.16(\mathrm{~m}, 2 \mathrm{H}), 7.04(\mathrm{~d}, J=9.5 \mathrm{~Hz}, 1 \mathrm{H}), 6.76-6.73(\mathrm{~m}, 4 \mathrm{H}), 6.67(\mathrm{~s}, 1 \mathrm{H})$, $4.14(\mathrm{t}, J=6.3 \mathrm{~Hz}, 1 \mathrm{H}), 3.70(\mathrm{~s}, 6 \mathrm{H}), 3.60(\mathrm{~s}, 3 \mathrm{H}), 3.34(\mathrm{~s}, 3 \mathrm{H}), 3.26(\mathrm{~s}, 3 \mathrm{H}), 3.18$ $(\mathrm{dd}, J=14.5,5.2 \mathrm{~Hz}, 1 \mathrm{H}), 3.10(\mathrm{dd}, J=14.4,7.7 \mathrm{~Hz}, 1 \mathrm{H}) ;{ }^{13} \mathrm{C}\left\{{ }^{1} \mathrm{H}\right\}$ NMR $\left(\mathrm{CDCl}_{3}, 100\right.$ $\mathrm{MHz}) \delta 171.1,170.6,157.9,153.6,137.3,136.4,132.7,129.4,128.4,128.3,127.7$, 127.2, 127.1, 118.4, 113.6, 111.7, 109.9, 101.7, 61.6, 56.1, 55.3, 52.5, 52.3, 42.0, 37.8, 32.9; HRMS (ESI, m/z): $\left[\mathrm{M}+\mathrm{Na}^{+} \mathrm{C}_{30} \mathrm{H}_{31} \mathrm{NNaO}_{6}\right.$ Calcd. 524.2049, Found 524.2042 .

Dimethyl phenylmalonate (5b):

2-(2-(5-bromo-1-methyl-1H-indol-3-yl)-2-phenylethyl)-2-<smiles>COC(C)(CC(c1ccccc1)c1ccccc1)c1cn(C)c2ccc(Br)cc12</smiles>

Following the general procedure, D-A cyclopropane $(1 \mathrm{~b}, 0.023 \mathrm{~g}, 0.1 \mathrm{mmol})$ was subjected to 1,3-bisarylation using 5-bromo-1-methyl indole and $\mathrm{Ph}_{3} \mathrm{BiCl}_{2}$ as arene sources and the crude product was purified via silica gel column chromatography (EtOAc: Hexane 2:8), which afforded compound $\mathbf{5 b}$ in $76 \%$ yield $(0.039 \mathrm{~g})$ as colourless oil. $R_{f} 0.35$ (EtOAc: Hexane 3:7); ${ }^{1} \mathrm{H}$ NMR $\left(\mathrm{CDCl}_{3}, 400 \mathrm{MHz}\right) \delta 7.53(\mathrm{~d}, \mathrm{~J}=$ $7.1 \mathrm{~Hz}, 2 \mathrm{H}), 7.34-7.28(\mathrm{~m}, 4 \mathrm{H}), 7.24-7.16(\mathrm{~m}, 5 \mathrm{H}), 7.11(\mathrm{t}, J=7.0 \mathrm{~Hz}, 1 \mathrm{H}), 7.03(\mathrm{~d}$, $J=8.6 \mathrm{~Hz}, 1 \mathrm{H}), 6.71(\mathrm{~s}, 1 \mathrm{H}), 4.17(\mathrm{t}, J=6.4 \mathrm{~Hz}, 1 \mathrm{H}), 3.62(\mathrm{~s}, 3 \mathrm{H}), 3.35(\mathrm{~s}, 3 \mathrm{H}), 3.23$ (s, 3H), $3.20-3.08(\mathrm{~m}, 2 \mathrm{H}) ;{ }^{13} \mathrm{C}\left\{{ }^{1} \mathrm{H}\right\}$ NMR $\left(\mathrm{CDCl}_{3}, 100 \mathrm{MHz}\right) \delta 171.0,170.5,144.1$, 137.0, 135.9, 128.5 (2), 128.3 (2), 127.9, 127.8, 126.4, 124.6, 122.1, 118.2, 112.4, 110.9, 61.6, 52.5, 52.3, 42.3, 38.4, 33.0; HRMS (ESI-TOF) m/z: $[\mathrm{M}+\mathrm{Na}]^{+}$ $\mathrm{C}_{28} \mathrm{H}_{26} \mathrm{BrNNaO}_{4}$ Calcd. 542.0943, Found 542.0934. 
Dimethyl 2-(2-(6-methoxy-1-methyl-1H-indol-3-yl)-2-(4-methoxyphenyl)ethyl)-2phenylmalonate $(5 c)$ :

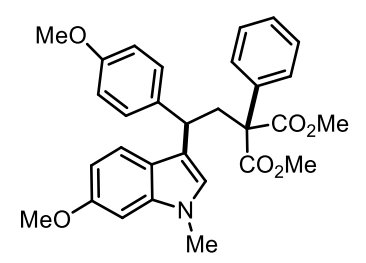

Following the general procedure, D-A cyclopropane $(1 \mathrm{a}, 0.027 \mathrm{~g}, 0.1 \mathrm{mmol})$ was subjected to 1,3-bisarylation using 6- methoxy-1-methyl indole and $\mathrm{Ph}_{3} \mathrm{BiCl}_{2}$ as arene sources and the crude product was purified via silica gel column chromatography (EtOAc: Hexane 2:8), which afforded compound $5 \mathrm{c}$ in $82 \%$ yield $(0.041 \mathrm{~g})$ as colourless oil. $R_{f} 0.30$ (EtOAc: Hexane 3:7); FT-IR $\left(\mathrm{v} \mathrm{cm}^{-1}\right): 2950,1734$, 1582, 1510, 1246; ${ }^{1} \mathrm{H}$ NMR $\left(\mathrm{CDCl}_{3}, 400 \mathrm{MHz}\right) \delta 7.57$ (d, $\left.J=7.5 \mathrm{~Hz}, 2 \mathrm{H}\right), 7.36-7.30$ $(\mathrm{m}, 3 \mathrm{H}), 7.20(\mathrm{~d}, J=8.3 \mathrm{~Hz}, 3 \mathrm{H}), 6.78(\mathrm{~d}, J=8.4 \mathrm{~Hz}, 2 \mathrm{H}), 6.67-6.64(\mathrm{~m}, 3 \mathrm{H}), 4.19(\mathrm{t}$, $J=6.4 \mathrm{~Hz}, 1 \mathrm{H}), 3.83(\mathrm{~s}, 3 \mathrm{H}), 3.75(\mathrm{~s}, 3 \mathrm{H}), 3.64(\mathrm{~s}, 3 \mathrm{H}), 3.38(\mathrm{~s}, 3 \mathrm{H}), 3.30(\mathrm{~s}, 3 \mathrm{H})$, $3.23(\mathrm{dd}, J=14.5,5.6 \mathrm{~Hz}, 1 \mathrm{H}), 3.15(\mathrm{dd}, J=14.4,7.3 \mathrm{~Hz}, 1 \mathrm{H}) ;{ }^{13} \mathrm{C}\left\{{ }^{1} \mathrm{H}\right\} \mathrm{NMR}$ $\left(\mathrm{CDCl}_{3}, 100 \mathrm{MHz}\right) \delta 171.1,170.7,157.9,156.4,138.0,137.2,136.8,129.3,128.4$, 128.3, 127.7, 125.3, 121.4 (2), 119.0, 113.6, 108.6, 92.8, 61.6, 55.8, 55.3, 52.5, 52.3, 42.1, 37.9, 32.7; HRMS (ESI-TOF) m/z: $[\mathrm{M}+\mathrm{Na}]^{+} \quad \mathrm{C}_{30} \mathrm{H}_{31} \mathrm{NNaO}_{6}$ Calcd. 524.2049, Found 524.2045.

Dimethyl 2-(2-(4-(tert-butyl)phenyl)-2-(1,2-dimethyl-1H-indol-3-yl)ethyl)-2phenylmalonate $(\mathbf{5 d})$ :<smiles>COC(C)(OC)c1ccccc1C(CC(C)(C)C)c1ccccc1</smiles>

Following the general procedure, D-A cyclopropane $(1 \mathrm{a}, 0.031 \mathrm{~g}, 0.1 \mathrm{mmol})$ was subjected to 1,3-bisarylation using 1,2-dimethyl indole and $\mathrm{Ph}_{3} \mathrm{BiCl}_{2}$ as arene sources and the crude product was purified via silica gel column chromatography (EtOAc: Hexane 2:8), which afforded compound $\mathbf{5 d}$ in $82 \%$ yield $(0.042 \mathrm{~g})$ as colourless oil. $R_{f} 0.35$ (EtOAc: Hexane 3:7); ${ }^{1} \mathrm{H}$ NMR $\left(\mathrm{CDCl}_{3}, 400 \mathrm{MHz}\right) \delta 7.63(\mathrm{~d}, \mathrm{~J}=$ $7.8 \mathrm{~Hz}, 1 \mathrm{H}), 7.54(\mathrm{~d}, J=4.4 \mathrm{~Hz}, 2 \mathrm{H}), 7.25-7.19(\mathrm{~m}, 5 \mathrm{H}), 7.16-7.10(\mathrm{~m}, 3 \mathrm{H}), 7.03(\mathrm{t}$, $J=7.5 \mathrm{~Hz}, 1 \mathrm{H}), 6.96(\mathrm{t}, J=7.4 \mathrm{~Hz}, 1 \mathrm{H}), 4.15(\mathrm{dd}, J=8.5,4.3 \mathrm{~Hz}, 1 \mathrm{H}), 3.60-3.55$ (m, 1H), 3.51 (s, 3H), 3.35 (dd, $J=14.4,4.4 \mathrm{~Hz}, 1 \mathrm{H}), 3.24(\mathrm{~s}, 3 \mathrm{H}), 2.91(\mathrm{~s}, 3 \mathrm{H}), 2.09$ $(\mathrm{s}, 3 \mathrm{H}), 1.17(\mathrm{~s}, 9 \mathrm{H}) ;{ }^{13} \mathrm{C}\left\{{ }^{1} \mathrm{H}\right\}$ NMR $\left(\mathrm{CDCl}_{3}, 100 \mathrm{MHz}\right) \delta 171.2,170.4,148.4,142.5$, $137.1,136.6,133.9,128.6,128.2,127.6,127.4,126.6,125.2,120.2,120.2,118.8$, 112.8, 108.6, 61.4, 52.4, 51.7, 40.1, 37.6, 34.4, 31.5, 29.6, 10.8; HRMS (ESI-TOF) $\mathrm{m} / \mathrm{z}:[\mathrm{M}+\mathrm{Na}]^{+} \mathrm{C}_{33} \mathrm{H}_{37} \mathrm{NNaO}_{4}$ Calcd. 534.2620, Found 534.2622. 


\section{Dimethyl 2-(2,2-bis(4-methoxyphenyl)ethyl)-2-phenyImalonate (5e):}<smiles>COc1ccc(C(CC(C)(O)c2ccccc2)c2ccc(OC)cc2)cc1</smiles>

This reaction was carried out following the general procedure, except DCM was used as solvent and it was heated at $50{ }^{\circ} \mathrm{C}$ on an oil bath. Accordingly, D-A cyclopropane (1a, $0.027 \mathrm{~g}, 0.1 \mathrm{mmol}$ ) was subjected to 1,3-bisarylation using anisole and $\mathrm{Ph}_{3} \mathrm{BiCl}_{2}$ as arene sources and the crude product was purified via silica gel column chromatography (EtOAc: Hexane 3:7), which afforded compound $5 \mathbf{e}$ in $71 \%$ yield $(0.032 \mathrm{~g})$ as colourless oil. $R_{f} 0.35$ (EtOAc: Hexane $\left.3: 7\right) ;{ }^{1} \mathrm{H}$ NMR $\left(\mathrm{CDCl}_{3}, 400 \mathrm{MHz}\right)$ $\delta 7.50(\mathrm{~d}, J=7.0 \mathrm{~Hz}, 2 \mathrm{H}), 7.35-7.29(\mathrm{~m}, 3 \mathrm{H}), 7.12(\mathrm{~d}, J=8.5 \mathrm{~Hz}, 4 \mathrm{H}), 6.77(\mathrm{~d}, J=$ $8.4 \mathrm{~Hz}, 4 \mathrm{H}), 3.92(\mathrm{t}, J=6.1 \mathrm{~Hz}, 1 \mathrm{H}), 3.74(\mathrm{~s}, 6 \mathrm{H}), 3.37(\mathrm{~s}, 6 \mathrm{H}), 3.17(\mathrm{~d}, J=6.2 \mathrm{~Hz}$, $2 \mathrm{H}) ;{ }^{13} \mathrm{C}\left\{{ }^{1} \mathrm{H}\right\}$ NMR $\left(\mathrm{CDCl}_{3}, 100 \mathrm{MHz}\right) \delta 170.8,158.0,137.5,136.8,128.8,128.5$, 128.3, 127.8, 113.9, 61.7, 55.4, 52.5, 45.6, 41.5; HRMS (ESI-TOF) m/z: [M+Na] ${ }^{+}$ $\mathrm{C}_{27} \mathrm{H}_{28} \mathrm{NaO}_{6}$ Calcd. 471.1784, Found 471.1778.

\section{Dimethyl 2-(2-(2-methoxy-5-methylphenyl)-2-(4-methoxyphenyl)ethyl)-2- phenylmalonate (5f):}<smiles>COc1ccc(C(CC(C)(O[Na])c2ccccc2)c2cccc(OC)c2)cc1</smiles>

This reaction was carried out following the general procedure, except DCM was used as solvent and it was heated at $50{ }^{\circ} \mathrm{C}$ on an oil bath. Accordingly, D-A cyclopropane (1a, $0.027 \mathrm{~g}, 0.1 \mathrm{mmol}$ ) was subjected to 1,3-bisarylation using 4-methyl anisole and $\mathrm{Ph}_{3} \mathrm{BiCl}_{2}$ as arene sources and the crude product was purified via silica gel column chromatography (EtOAc: Hexane 2:8), which afforded compound $\mathbf{5 f}$ in $67 \%$ yield $(0.031 \mathrm{~g})$ as colourless oil. $R_{f} 0.35$ (EtOAc: Hexane $\left.3: 7\right) ;{ }^{1} \mathrm{H}$ NMR $\left(\mathrm{CDCl}_{3}, 400 \mathrm{MHz}\right)$ $\delta 7.50(\mathrm{~d}, J=7.5 \mathrm{~Hz}, 2 \mathrm{H}), 7.37-7.27(\mathrm{~m}, 3 \mathrm{H}), 7.18(\mathrm{~d}, J=8.4 \mathrm{~Hz}, 2 \mathrm{H}), 7.07(\mathrm{~s}, 1 \mathrm{H})$, $6.92-6.86(\mathrm{~m}, 1 \mathrm{H}), 6.75(\mathrm{~d}, J=8.4 \mathrm{~Hz}, 2 \mathrm{H}), 6.65(\mathrm{~d}, J=8.2 \mathrm{~Hz}, 1 \mathrm{H}), 4.38(\mathrm{t}, J=6.3$ $\mathrm{Hz}, 1 \mathrm{H}), 3.73(\mathrm{~s}, 3 \mathrm{H}), 3.66(\mathrm{~s}, 3 \mathrm{H}), 3.42(\mathrm{~s}, 3 \mathrm{H}), 3.33(\mathrm{~s}, 3 \mathrm{H}), 3.17$ (d, J = $6.3 \mathrm{~Hz}$, $2 \mathrm{H}), 2.25(\mathrm{~s}, 3 \mathrm{H}) ;{ }^{13} \mathrm{C}\left\{{ }^{1} \mathrm{H}\right\}$ NMR $\left(\mathrm{CDCl}_{3}, 100 \mathrm{MHz}\right) \delta 170.9,170.8,157.8,154.6$, 136.9, 136.8, 133.4, 129.6, 129.3, 128.7, 128.6, 128.1, 127.6, 127.5, 113.5, 111.1, 61.8, 55.7, 55.3, 52.4, 52.3, 40.5, 38.6, 20.8; HRMS (ESI-TOF) m/z: [M+Na] ${ }^{+}$ $\mathrm{C}_{28} \mathrm{H}_{30} \mathrm{NaO}_{6}$ Calcd. 485.1940, Found 485.1933. 


\section{phenylmalonate $(\mathbf{5 g})$ :}<smiles>COc1ccc(C(Cc2ccccc2)c2c(OC)cc(OC)cc2OC(C)(C)C)cc1</smiles>

This reaction was carried out following the general procedure, except DCM was used as solvent and it was heated at $50{ }^{\circ} \mathrm{C}$ on an oil bath. Accordingly, D-A cyclopropane $(1 \mathrm{a}, 0.027 \mathrm{~g}, 0.1 \mathrm{mmol})$ was subjected to 1,3-bisarylation using 1, 3, 5trimethxoybenzene and $\mathrm{Ph}_{3} \mathrm{BiCl}_{2}$ as arene sources and the crude product was purified via silica gel column chromatography (EtOAc: Hexane 2:8), which afforded compound $5 \mathrm{~g}$ in $80 \%$ yield $(0.041 \mathrm{~g})$ as colourless oil. $R_{f} 0.20$ (EtOAc: Hexane 3:7); ${ }^{1} \mathrm{H}$ NMR $\left(\mathrm{CDCl}_{3}, 400 \mathrm{MHz}\right) \delta 7.51(\mathrm{~d}, J=5.2 \mathrm{~Hz}, 2 \mathrm{H}), 7.32-7.24(\mathrm{~m}, 5 \mathrm{H}), 6.72(\mathrm{~d}, J=$ $8.4 \mathrm{~Hz}, 2 \mathrm{H}), 6.06(\mathrm{~s}, 2 \mathrm{H}), 4.64(\mathrm{t}, \mathrm{J}=5.9 \mathrm{~Hz}, 1 \mathrm{H}), 3.77(\mathrm{~s}, 3 \mathrm{H}), 3.74-3.73(\mathrm{~m}, 9 \mathrm{H})$, $3.42(\mathrm{~s}, 3 \mathrm{H}), 3.37(\mathrm{~m}, 4 \mathrm{H}), 3.32(\mathrm{dd}, \mathrm{J}=15.0,5.8 \mathrm{~Hz}, 1 \mathrm{H}) ;{ }^{13} \mathrm{C}\left\{{ }^{1} \mathrm{H}\right\}$ NMR $\left(\mathrm{CDCl}_{3}, 100\right.$ $\mathrm{MHz}) \delta 171.2,171.1,159.6,157.4,137.4,137.0,129.3,128.7,127.9,127.4,114.5$, 113.1, 91.1 62.0, 55.3, 55.3, 52.4, 52.3, 38.4, 34.4; HRMS (ESI-TOF) m/z: [M+Na] $\mathrm{C}_{29} \mathrm{H}_{32} \mathrm{NaO}_{8}$ Calcd. 531.1995, Found 531.1989.

Dimethyl
phenylmalonate (

This reaction was carried out following the general procedure, except DCM was used as solvent and it was heated at $50^{\circ} \mathrm{C}$ on an oil bath. Accordingly, D-A cyclopropane (1a, $0.027 \mathrm{~g}, 0.1 \mathrm{mmol}$ ) was subjected to 1,3-bisarylation using $N, N$-dimethylaniline and $\mathrm{Ph}_{3} \mathrm{BiCl}_{2}$ as arene sources and the crude product was purified via silica gel column chromatography (EtOAc: Hexane 2:8), which afforded compound $5 \mathrm{~h}$ in $69 \%$ yield $(0.032 \mathrm{~g})$ as colourless oil. $R_{f} 0.35$ (EtOAc: Hexane $\left.3: 7\right) ;{ }^{1} \mathbf{H}$ NMR $\left(\mathrm{CDCl}_{3}, 400\right.$ $\mathrm{MHz}) \delta 7.51$ (d, J = $7.5 \mathrm{~Hz}, 2 \mathrm{H}), 7.35-7.29(\mathrm{~m}, 3 \mathrm{H}), 7.12(\mathrm{~d}, J=8.4 \mathrm{~Hz}, 2 \mathrm{H}), 7.07$ (d, J = 8.4 Hz, 2H), $6.76(\mathrm{~d}, J=8.3 \mathrm{~Hz}, 2 \mathrm{H}), 6.63$ (d, J = 8.4 Hz, 2H), 3.86 (t, J = 6.2 $\mathrm{Hz}, 1 \mathrm{H}), 3.73(\mathrm{~s}, 3 \mathrm{H}), 3.37(\mathrm{~s}, 3 \mathrm{H}), 3.35(\mathrm{~s}, 3 \mathrm{H}), 3.17-3.15(\mathrm{~m}, 2 \mathrm{H}), 2.87(\mathrm{~s}, 6 \mathrm{H}) ;{ }^{13} \mathrm{C}$ $\left\{{ }^{1} \mathrm{H}\right\}$ NMR $\left(\mathrm{CDCl}_{3}, 100 \mathrm{MHz}\right) \delta 170.9,170.8,157.9,149.2,137.9,136.9,133.6$, 128.9, 128.6, 128.5, 128.3, 127.8, 113.8, 113.0, 61.8, 55.4, 52.5, 52.5, 45.4, 41.5, 40.9; HRMS (ESI-TOF) m/z: [M+H] ${ }^{+} \mathrm{C}_{28} \mathrm{H}_{32} \mathrm{NO}_{5}$ Calcd. 462.2280, Found 462.2276. 
<smiles>COc1ccc(C(CC(C)(C)C)(c2ccccc2)c2ccc(N(C=O)C=O)cc2)cc1</smiles>

This reaction was carried out following the general procedure, except DCM was used as solvent and it was heated at $50{ }^{\circ} \mathrm{C}$ on an oil bath. Accordingly, D-A cyclopropane $(1 \mathrm{a}, \quad 0.027 \mathrm{~g}, \quad 0.1 \mathrm{mmol})$ was subjected to 1,3-bisarylation using (4morpholino)benzene and $\mathrm{Ph}_{3} \mathrm{BiCl}_{2}$ as arene sources and the crude product was purified via silica gel column chromatography (EtOAc: Hexane 3:7), which afforded compound $5 \mathbf{i}$ in $80 \%$ yield $(0.040 \mathrm{~g})$ as colourless oil. $R_{f} 0.35$ (EtOAc: Hexane $4: 6$ ); ${ }^{1} \mathrm{H}$ NMR $\left(\mathrm{CDCl}_{3}, 400 \mathrm{MHz}\right) \delta 7.49(\mathrm{~d}, J=7.1 \mathrm{~Hz}, 2 \mathrm{H}), 7.34-7.29(\mathrm{~m}, 3 \mathrm{H}), 7.11$ (dd, $J$ $=8.4,4.8 \mathrm{~Hz}, 4 \mathrm{H}), 6.77(\mathrm{dd}, J=8.5,6.6 \mathrm{~Hz}, 4 \mathrm{H}), 3.89(\mathrm{t}, J=6.1 \mathrm{~Hz}, 1 \mathrm{H}), 3.82(\mathrm{t}, J=$ $4.8 \mathrm{~Hz}, 4 \mathrm{H}), 3.74(\mathrm{~s}, 3 \mathrm{H}), 3.37(\mathrm{~s}, 3 \mathrm{H}), 3.36(\mathrm{~s}, 3 \mathrm{H}), 3.16(\mathrm{~d}, J=6.1 \mathrm{~Hz}, 2 \mathrm{H}), 3.07(\mathrm{t}$, $J=4.9 \mathrm{~Hz}, 4 \mathrm{H}) ;{ }^{13} \mathrm{C}\left\{{ }^{1} \mathrm{H}\right\}$ NMR $\left(\mathrm{CDCl}_{3}, 100 \mathrm{MHz}\right) \delta 170.8,170.8,158.0,149.7,137.5$, 137.0, 136.8, 128.9, 128.6, 128.5, 128.3, 127.8, 115.9, 113.9, 67.1, 61.7, 55.4, 52.5, 52.5, 49.7, 45.5, 41.4; HRMS (ESI-TOF) m/z: $[\mathrm{M}+\mathrm{H}]^{+} \mathrm{C}_{30} \mathrm{H}_{34} \mathrm{NO}_{6}$ Calcd. 504.2381, Found 504.2379.

\section{Dimethyl 2-(2-(4-methoxyphenyl)-2-(1-methyl-1H-indol-3-yl)ethyl)-2-(p- tolyl)malonate (5j):}<smiles>COC(=O)C(CC(c1ccc(OC)cc1)C(c1ccc(OC)cc1)c1ccccc1[N+](=O)[O-])(c1ccc(C)cc1)C(C)(O)O</smiles>

Following the general procedure, D-A cyclopropane $(1 \mathrm{a}, 0.027 \mathrm{~g}, 0.1 \mathrm{mmol})$ was subjected to 1,3-bisarylation using 1-methyl indole and $(p-\mathrm{Me})_{3} \mathrm{BiCl}_{2}$ as arene sources and the crude product was purified via silica gel column chromatography (EtOAc: Hexane 2:8), which afforded compound $\mathbf{5 j}$ in $70 \%$ yield $(0.034 \mathrm{~g})$ as colourless oil. $R_{f} 0.35$ (EtOAc: Hexane 3:7); ${ }^{1} \mathrm{H}$ NMR $\left(\mathrm{CDCl}_{3}, 400 \mathrm{MHz}\right) \delta 7.47-7.45$ $(\mathrm{m}, 2 \mathrm{H}), 7.38(\mathrm{~d}, J=8.0 \mathrm{~Hz}, 1 \mathrm{H}), 7.24-7.21(\mathrm{~m}, 3 \mathrm{H}), 7.17-7.14(\mathrm{~m}, 3 \mathrm{H}), 6.99(\mathrm{t}, J=$ $7.3 \mathrm{~Hz}, 1 \mathrm{H}), 6.80-6.77(\mathrm{~m}, 3 \mathrm{H}), 4.25(\mathrm{t}, J=6.4 \mathrm{~Hz}, 1 \mathrm{H}), 3.75(\mathrm{~s}, 3 \mathrm{H}), 3.70(\mathrm{~s}, 3 \mathrm{H})$, $3.36(\mathrm{~s}, 3 \mathrm{H}), 3.28(\mathrm{~s}, 3 \mathrm{H}), 3.27-3.23(\mathrm{~m}, 1 \mathrm{H}), 3.17(\mathrm{dd}, J=14.3,7.3 \mathrm{~Hz}, 1 \mathrm{H}), 2.35$ $(\mathrm{s}, 3 \mathrm{H}) ;{ }^{13} \mathrm{C}\left\{{ }^{1} \mathrm{H}\right\}$ NMR $\left(\mathrm{CDCl}_{3}, 100 \mathrm{MHz}\right) \delta 171.2,170.8,157.9,137.4,137.3,136.9$, 134.2, 129.3, 129.0, 128.3, 127.0, 126.4, 121.6, 119.8, 119.0, 118.8, 113.6, 109.1, $61.3,55.4,52.4,52.3,41.9,37.8,32.8,21.1$; HRMS (ESI-TOF) $\mathrm{m} / \mathrm{z}:[\mathrm{M}+\mathrm{Na}]^{+}$ $\mathrm{C}_{30} \mathrm{H}_{31} \mathrm{NNaO}_{5}$ Calcd. 508.2100, Found 508.2097. 
Dimethyl 2-(4-fluorophenyl)-2-(2-(4-methoxyphenyl)-2-(1-methyl-1H-indol-3yl)ethyl)malonate (5I):<smiles>COc1ccc(C(CC(C)(OC)c2ccc(F)cc2)c2cn(C)c3ccccc23)cc1</smiles>

Following the general procedure, D-A cyclopropane $(1 \mathrm{a}, 0.027 \mathrm{~g}, 0.1 \mathrm{mmol}$ ) was subjected to 1,3-bisarylation using 1-methyl indole and $\left(p-\mathrm{FC}_{6} \mathrm{H}_{4}\right)_{3} \mathrm{BiCl}_{2}$ as arene sources and the crude product was purified via silica gel column chromatography (EtOAc: Hexane 3:7), which afforded compound $5 \mathrm{I}$ in $57 \%$ yield $(0.028 \mathrm{~g})$ as colourless oil. $R_{f} 0.35$ (EtOAc: Hexane 3:7); FT-IR $\left(\mathrm{v} \mathrm{cm}^{-1}\right): 2952,1733,1608,1510$, 1233; ${ }^{1} \mathrm{H}$ NMR $\left(\mathrm{CDCl}_{3}, 400 \mathrm{MHz}\right) \delta 7.56-7.52(\mathrm{~m}, 2 \mathrm{H}), 7.36(\mathrm{~d}, \mathrm{~J}=8.0 \mathrm{~Hz}, 1 \mathrm{H}), 7.23-$ $7.13(\mathrm{~m}, 4 \mathrm{H}), 7.02-6.98(\mathrm{~m}, 3 \mathrm{H}), 6.78-6.76(\mathrm{~m}, 3 \mathrm{H}), 4.18(\mathrm{t}, \mathrm{J}=6.4 \mathrm{~Hz}, 1 \mathrm{H}), 3.74(\mathrm{~s}$, $3 \mathrm{H}), 3.70(\mathrm{~s}, 3 \mathrm{H}), 3.38$ (s, 3H), 3.30 (s, 3H), 3.25 (dd, J = 14.5, $5.5 \mathrm{~Hz}, 1 \mathrm{H}$ ), 3.15 (dd, $J=14.5,7.3 \mathrm{~Hz}, 1 \mathrm{H}) ;{ }^{13} \mathrm{C}\left\{{ }^{1} \mathrm{H}\right\}$ NMR $\left(\mathrm{CDCl}_{3}, 100 \mathrm{MHz}\right) \delta 171.0,170.6,162.2(\mathrm{~d}, \mathrm{~J}=$ $246 \mathrm{~Hz}), 157.9,137.2,136.5,132.7(\mathrm{~d}, J=3 \mathrm{~Hz}), 130.3(\mathrm{~d}, \mathrm{~J}=8 \mathrm{~Hz}), 129.3,126.8$, 126.3, 121.7, 119.7, 118.9, 118.8, 115.1(d, $J=21 \mathrm{~Hz}), 113.7,109.2,61.0,55.8$, 52.6, 52.5, 42.00, 37.8, 32.8; HRMS (ESI-TOF) m/z: [M+Na] ${ }^{+} \mathrm{C}_{29} \mathrm{H}_{28} \mathrm{FNNaO}_{5} \mathrm{Calcd}$ 512.1849, Found 512.1845.

Dimethyl 2-(4-chlorophenyl)-2-(2-(4-methoxyphenyl)-2-(1-methyl-1H-indol-3yl)ethyl)malonate $(5 \mathrm{~m})$ :<smiles>COCC(CC(c1ccc(OC)cc1)c1cn(C)c2ccccc12)(c1ccc(Cl)cc1)C(C)(O)O</smiles>

Following the general procedure, D-A cyclopropane $(1 \mathrm{a}, 0.027 \mathrm{~g}, 0.1 \mathrm{mmol})$ was subjected to 1,3-bisarylation using 1-methyl indole and $\left(p-\mathrm{ClC}_{6} \mathrm{H}_{4}\right)_{3} \mathrm{BiCl}_{2}$ as arene sources and the crude product was purified via silica gel column chromatography (EtOAc: Hexane 2:8), which afforded compound $5 \mathrm{~m}$ in $65 \%$ yield $(0.033 \mathrm{~g})$ as colourless oil. $R_{f} 0.35$ (EtOAc: Hexane 3:7); ${ }^{1} \mathrm{H}$ NMR $\left(\mathrm{CDCl}_{3}, 400 \mathrm{MHz}\right) \delta 7.52$ (d, J = $8.4 \mathrm{~Hz}, 2 \mathrm{H}), 7.41(\mathrm{~d}, J=8.0 \mathrm{~Hz}, 1 \mathrm{H}), 7.30(\mathrm{~d}, J=8.6 \mathrm{~Hz}, 2 \mathrm{H}), 7.24-7.17(\mathrm{~m}, 4 \mathrm{H})$, $7.03(\mathrm{t}, J=7.4 \mathrm{~Hz}, 1 \mathrm{H}), 6.81-6.79(\mathrm{~m}, 3 \mathrm{H}), 4.21(\mathrm{t}, J=6.5 \mathrm{~Hz}, 1 \mathrm{H}), 3.78(\mathrm{~s}, 3 \mathrm{H})$, $3.73(\mathrm{~s}, 3 \mathrm{H}), 3.42(\mathrm{~s}, 3 \mathrm{H}), 3.36(\mathrm{~s}, 3 \mathrm{H}), 3.28(\mathrm{dd}, J=14.6,5.7 \mathrm{~Hz}, 1 \mathrm{H}), 3.18(\mathrm{dd}, J=$ 14.6, 7.3 Hz, 1H); ${ }^{13} \mathrm{C}\left\{{ }^{1} \mathrm{H}\right\}$ NMR $\left(\mathrm{CDCl}_{3}, 100 \mathrm{MHz}\right) \delta 170.7,170.4,158.0,137.3$, 136.5, 135.5, 133.7, 129.9, 129.2, 128.4, 126.8, 126.4, 121.7, 119.7, 118.9, 118.7, 113.7, 109.2, 61.2, 55.4, 52.6, 52.5, 41.9, 37.9, 32.8; HRMS (ESI-TOF) m/z: [M+Na] ${ }^{+}$ $\mathrm{C}_{29} \mathrm{H}_{28} \mathrm{CINNaO}$ Calcd. 528.1554, Found 528.1547. 
<smiles>COc1ccc(C(CC(C)(OC)c2ccc(C)cc2)c2ccccc2N(C)C)cc1</smiles>

Following the general procedure, D-A cyclopropane $(1 \mathrm{a}, 0.027 \mathrm{~g}, 0.1 \mathrm{mmol})$ was subjected to 1,3-bisarylation using 1-methyl indole and $\left(p-\mathrm{CF}_{3} \mathrm{C}_{6} \mathrm{H}_{4}\right)_{3} \mathrm{BiCl}_{2}$ as arene sources and the crude product was purified via silica gel column chromatography (EtOAc: Hexane 2:8), which afforded compound $5 \mathrm{n}$ in $55 \%$ yield $(0.030 \mathrm{~g})$ as colourless oil. $R_{f} 0.35$ (EtOAc: Hexane 3:7); ${ }^{1} \mathrm{H}$ NMR $\left(\mathrm{CDCl}_{3}, 400 \mathrm{MHz}\right) \delta 7.66$ (d, J = $8.3 \mathrm{~Hz}, 2 \mathrm{H}), 7.52(\mathrm{~d}, J=8.3 \mathrm{~Hz}, 2 \mathrm{H}), 7.34(\mathrm{~d}, J=7.9 \mathrm{~Hz}, 1 \mathrm{H}), 7.22-7.13(\mathrm{~m}, 4 \mathrm{H})$, $6.99(\mathrm{t}, J=7.4 \mathrm{~Hz}, 1 \mathrm{H}), 6.75(\mathrm{~d}, J=9.3 \mathrm{~Hz}, 3 \mathrm{H}), 4.18(\mathrm{t}, J=6.5 \mathrm{~Hz}, 1 \mathrm{H}), 3.74$ (s, $3 \mathrm{H}), 3.68(\mathrm{~s}, 3 \mathrm{H}), 3.43(\mathrm{~s}, 3 \mathrm{H}), 3.36(\mathrm{~s}, 3 \mathrm{H}), 3.27$ (dd, J = 14.6, $5.7 \mathrm{~Hz}, 1 \mathrm{H}), 3.18$ (dd, $J=14.5,7.4 \mathrm{~Hz}, 1 \mathrm{H}) ;{ }^{13} \mathrm{C}\left\{{ }^{1} \mathrm{H}\right\}$ NMR $\left(\mathrm{CDCl}_{3}, 100 \mathrm{MHz}\right) \delta 170.5,170.2,158.0,140.9$, 137.3, 136.4, 129.8 (q, $J=134.2 \mathrm{~Hz}), 129.2,128.9,126.8,126.4,125.0$ (q, $J=$ $3.7 \mathrm{~Hz}), 124.1(\mathrm{q}, J=270.5 \mathrm{~Hz}), 121.7,119.6,119.0,113.7,109.3,61.6,55.4,52.8$, 52.7, 42.0, 37.9, 32.8; HRMS (ESI-TOF) m/z: $[\mathrm{M}+\mathrm{Na}]^{+} \mathrm{C}_{30} \mathrm{H}_{28} \mathrm{~F}_{3} \mathrm{NNaO}_{5}$ Calcd. 562.1817, Found 562.1802.

Dimethyl 2-(2-(4-methoxyphenyl)-2-(1-methyl-1H-indol-3-yl)ethyl)-2-(3(trifluoromethyl)phenyl)malonate(5p):<smiles>COc1ccc(C(CC(C)(OC)c2cccc(C(F)(F)F)c2)c2cn(C)c3ccccc23)cc1</smiles>

Following the general procedure, D-A cyclopropane $(1 \mathrm{a}, 0.027 \mathrm{~g}, 0.1 \mathrm{mmol})$ was subjected to 1,3-bisarylation using 1-methyl indole and $\left(m-\mathrm{CF}_{3} \mathrm{C}_{6} \mathrm{H}_{4}\right)_{3} \mathrm{BiCl}_{2}$ as arene sources and the crude product was purified via silica gel column chromatography (EtOAc: Hexane 2:8), which afforded compound $5 p$ in $67 \%$ yield $(0.036 \mathrm{~g})$ as colourless oil. $R_{f} 0.35$ (EtOAc: Hexane 3:7); FT-IR $\left(\mathrm{v} \mathrm{cm}^{-1}\right): 2952,1736,1511,1247$, 1170, 1125; ${ }^{1} \mathrm{H}$ NMR $\left(\mathrm{CDCl}_{3}, 400 \mathrm{MHz}\right) \delta 7.93(\mathrm{~s}, 1 \mathrm{H}), 7.69$ (d, J = 8.0 Hz, 1H), 7.54 $(\mathrm{d}, J=7.7 \mathrm{~Hz}, 1 \mathrm{H}), 7.42-7.35(\mathrm{~m}, 2 \mathrm{H}), 7.23-7.14(\mathrm{~m}, 4 \mathrm{H}), 7.02-6.98(\mathrm{~m}, 1 \mathrm{H}), 6.78$ - $6.74(\mathrm{~m}, 3 \mathrm{H}), 4.16(\mathrm{t}, J=6.5 \mathrm{~Hz}, 1 \mathrm{H}), 3.75(\mathrm{~s}, 3 \mathrm{H}), 3.69(\mathrm{~s}, 3 \mathrm{H}), 3.42(\mathrm{~s}, 3 \mathrm{H}), 3.38$ $(\mathrm{s}, 3 \mathrm{H}), 3.28(\mathrm{dd}, J=14.6,5.8 \mathrm{~Hz}, 1 \mathrm{H}), 3.19(\mathrm{dd}, J=14.5,7.3 \mathrm{~Hz}, 1 \mathrm{H}) ;{ }^{13} \mathrm{C}\left\{{ }^{1} \mathrm{H}\right\}$ NMR $\left(\mathrm{CDCl}_{3}, 100 \mathrm{MHz}\right) \delta 170.5,170.3,158.0,138.1,137.3,136.3,131.8$ (2), 130.5 $(q, J=32.1 \mathrm{~Hz}), 129.2,128.7,126.8,126.5,125.5(q, J=4.1 \mathrm{~Hz}), 124.5(q, J=3.5$ $\mathrm{Hz}), 124.2$ (q, $J=270.9 \mathrm{~Hz}), 121.7,119.6,118.9$, 118.4, 113.7, 109.2, 61.5, 55.3, 52.6, 42.1, 37.9, 32.7; HRMS (ESI-TOF) m/z: [M+Na] ${ }^{+} \mathrm{C}_{30} \mathrm{H}_{28} \mathrm{~F}_{3} \mathrm{NNaO}_{5}$ Calcd. 562.1817, Found 562.1817. 


\section{tolyl)malonate $(\mathbf{5 q})$ :}<smiles>COc1ccc(C(CC(CO)(OC)c2cccc(OC)c2)c2cn(C)c3ccccc23)cc1</smiles>

Following the general procedure, D-A cyclopropane $(1 \mathrm{a}, 0.027 \mathrm{~g}, 0.1 \mathrm{mmol})$ was subjected to 1,3-bisarylation using 1-methyl indole and $\left(m-\mathrm{MeC}_{6} \mathrm{H}_{4}\right)_{3} \mathrm{BiCl}_{2}$ as arene sources and the crude product was purified via silica gel column chromatography (EtOAc: Hexane 2:8), which afforded compound $5 q$ in $72 \%$ yield $(0.035 \mathrm{~g})$ as colourless oil. $R_{f} 0.35$ (EtOAc: Hexane $\left.3: 7\right) ;{ }^{1} \mathrm{H}$ NMR $\left(\mathrm{CDCl}_{3}, 400 \mathrm{MHz}\right) \delta 7.38$ - 7.34 $(\mathrm{m}, 3 \mathrm{H}), 7.23-7.20(\mathrm{~m}, 4 \mathrm{H}), 7.15(\mathrm{~d}, J=7.2 \mathrm{~Hz}, 1 \mathrm{H}), 7.13-7.09(\mathrm{~m}, 1 \mathrm{H}), 6.98(\mathrm{t}, J=$ $7.4 \mathrm{~Hz}, 1 \mathrm{H}), 6.79-6.75(\mathrm{~m}, 3 \mathrm{H}), 4.24(\mathrm{t}, J=6.4 \mathrm{~Hz}, 1 \mathrm{H}), 3.75(\mathrm{~s}, 3 \mathrm{H}), 3.69(\mathrm{~s}, 3 \mathrm{H})$, $3.38(\mathrm{~s}, 3 \mathrm{H}), 3.30(\mathrm{~s}, 3 \mathrm{H}), 3.25-3.13(\mathrm{~m}, 2 \mathrm{H}), 2.31(\mathrm{~s}, 3 \mathrm{H}) ;{ }^{13} \mathrm{C}\left\{{ }^{1} \mathrm{H}\right\}$ NMR $\left(\mathrm{CDCl}_{3}, 100\right.$ $\mathrm{MHz}) \delta 171.2,170.8,157.9,137.9,137.3,137.1,136.8,129.3,129.0,128.5,128.2$, 126.5, 125.4, 121.6, 119.8, 119.0, 118.8, 113.6, 109.1, 61.6, 55.4, 52.5, 52.3, 42.3, 37.9, 32.8, 21.8; HRMS (ESI-TOF) m/z: [M+Na] ${ }^{+} \mathrm{C}_{30} \mathrm{H}_{31} \mathrm{NNaO}_{5}$ Calcd. 508.2100, Found 508.2099.

Dimethyl 2-(3-methoxyphenyl)-2-(2-(4-methoxyphenyl)-2-(1-methyl-1H-indol-3yl)ethyl)malonate (5r):<smiles>COc1ccc(C(CC(C)(OC)C(C)=O)c2cccc(OC)c2)cc1</smiles>

Following the general procedure, D-A cyclopropane $(1 \mathrm{a}, 0.027 \mathrm{~g}, 0.1 \mathrm{mmol})$ was subjected to 1,3-bisarylation using 1-methyl indole and $\left(m-\mathrm{OMeC}_{6} \mathrm{H}_{4}\right)_{3} \mathrm{BiCl}_{2}$ as arene sources and the crude product was purified via silica gel column chromatography (EtOAc: Hexane 2:8), which afforded compound $5 r$ in $76 \%$ yield $(0.038 \mathrm{~g})$ as colourless oil. $R_{f} 0.25$ (EtOAc: Hexane 3:7); ${ }^{1} \mathrm{H}$ NMR $\left(\mathrm{CDCl}_{3}, 400 \mathrm{MHz}\right) \delta 7.34$ (d, J = $8.0 \mathrm{~Hz}, 1 \mathrm{H}), 7.24(\mathrm{~d}, J=3.9 \mathrm{~Hz}, 1 \mathrm{H}), 7.23-7.18(\mathrm{~m}, 4 \mathrm{H}), 7.16-7.10(\mathrm{~m}, 2 \mathrm{H}), 6.97$ $(\mathrm{t}, J=7.4 \mathrm{~Hz}, 1 \mathrm{H}), 6.83(\mathrm{dd}, J=8.2,2.6 \mathrm{~Hz}, 1 \mathrm{H}), 6.78(\mathrm{~s}, 1 \mathrm{H}), 6.75(\mathrm{~d}, J=3.9 \mathrm{~Hz}$, $2 \mathrm{H}), 4.22(\mathrm{t}, J=6.5 \mathrm{~Hz}, 1 \mathrm{H}), 3.74(\mathrm{~s}, 3 \mathrm{H}), 3.73(\mathrm{~s}, 3 \mathrm{H}), 3.68(\mathrm{~s}, 3 \mathrm{H}), 3.37(\mathrm{~s}, 3 \mathrm{H})$, 3.31 (s, $3 \mathrm{H}), 3.23$ (dd, $J=14.5,5.7 \mathrm{~Hz}, 1 \mathrm{H}), 3.15(\mathrm{dd}, J=14.5,7.3 \mathrm{~Hz}, 1 \mathrm{H}) ;{ }^{13} \mathrm{C}\left\{{ }^{1} \mathrm{H}\right\}$ NMR $\left(\mathrm{CDCl}_{3}, 100 \mathrm{MHz}\right) \delta 171.0,170.6,159.4,157.9,138.7,137.3,136.7,129.3$, 129.2, 126.9, 126.4, 121.6, 120.5, 119.8, 118.8 (2), 114.6, 113.6, 113.1, 109.1, 61.5, $55.4, \quad 55.3, \quad 52.5, \quad 52.4, \quad 42.4, \quad 37.9, \quad 32.7$; HRMS (ESI-TOF) m/z: $[\mathrm{M}+\mathrm{Na}]^{+}$ $\mathrm{C}_{30} \mathrm{H}_{31} \mathrm{NNaO}_{6}$ Calcd. 524.2049, Found 524.2041. 
Dimethyl 2-(3-fluorophenyl)-2-(2-(4-methoxyphenyl)-2-(1-methyl-1H-indol-3yl)ethyl)malonate (5s):<smiles>COc1ccc(C(CC(C)(O[Na])c2cccc(F)c2)c2cn(C)c3ccccc23)cc1</smiles>

Following the general procedure, D-A cyclopropane $(1 \mathrm{a}, 0.027 \mathrm{~g}, 0.1 \mathrm{mmol})$ was subjected to 1,3-bisarylation using 1-methyl indole and $\left(m-\mathrm{FC}_{6} \mathrm{H}_{4}\right)_{3} \mathrm{BiCl}_{2}$ as arene sources and the crude product was purified via silica gel column chromatography (EtOAc: Hexane 2:8), which afforded compound $5 \mathrm{~s}$ in $61 \%$ yield $(0.030 \mathrm{~g})$ as brown solid. mp: $137-140^{\circ} \mathrm{C} R_{f} 0.20$ (EtOAc: Hexane 3:7); FT-IR $\left(v \mathrm{~cm}^{-1}\right)$ : 2919, 1735, 1587, 1510, 1245; ${ }^{1} \mathrm{H}$ NMR $\left(\mathrm{CDCl}_{3}, 400 \mathrm{MHz}\right) \delta 7.47(\mathrm{~d}, J=11.3 \mathrm{~Hz}, 1 \mathrm{H}), 7.38$ (d, J $=8.0 \mathrm{~Hz}, 1 \mathrm{H}), 7.32-7.28(\mathrm{~m}, 2 \mathrm{H}), 7.24-7.22(\mathrm{~m}, 3 \mathrm{H}), 7.17(\mathrm{t}, J=7.5 \mathrm{~Hz}, 1 \mathrm{H}), 7.01(\mathrm{t}$, $J=7.4 \mathrm{~Hz}, 2 \mathrm{H}), 6.81-6.79(\mathrm{~m}, 3 \mathrm{H}), 6.79(\mathrm{~s}, 2 \mathrm{H}), 4.21(\mathrm{t}, J=6.5 \mathrm{~Hz}, 1 \mathrm{H}), 3.76(\mathrm{~s}$, $3 \mathrm{H}), 3.71(\mathrm{~s}, 3 \mathrm{H}), 3.40(\mathrm{~s}, 3 \mathrm{H}), 3.34(\mathrm{~s}, 3 \mathrm{H}), 3.27(\mathrm{dd}, J=14.6,5.8 \mathrm{~Hz}, 1 \mathrm{H}), 3.18(\mathrm{dd}$, $J=14.4,7.4 \mathrm{~Hz}, 1 \mathrm{H}) ;{ }^{13} \mathrm{C}\left\{{ }^{1} \mathrm{H}\right\}$ NMR $\left(\mathrm{CDCl}_{3}, 100 \mathrm{MHz}\right) \delta 170.6,170.2,162.6(\mathrm{~d}$, $J=243.5), 157.9,139.5$ (d, J= 7.6), 137.2, 136.3, 129.6 (d, J=8.3), 129.2, 126.8, 126.4, 123.8, 123.7, 121.6, 119.6, 118.7 (d, J =30.4 Hz), 116.1 (d, J=23.6), 114.6 (d, $\mathrm{J}=20.9$ ), 113.6, 109.2, 61.2, 61.2, 55.3, 52.6, 52.5, 42.2, 37.8, 32.7; HRMS (ESITOF) m/z: [M+Na] ${ }^{+} \mathrm{C}_{29} \mathrm{H}_{28} \mathrm{FNNaO}_{5}$ Calcd. 512.1849, Found 512.1846.

Dimethyl 2-(3-chlorophenyl)-2-(2-(4-methoxyphenyl)-2-(1-methyl-1H-indol-3yl)ethyl)malonate (5t):<smiles>COc1ccc(C(CC(C)(OC)c2cccc(Cl)c2)c2cn(C)c3ccccc23)cc1</smiles>

Following the general procedure, D-A cyclopropane $(1 \mathrm{a}, 0.027 \mathrm{~g}, 0.1 \mathrm{mmol}$ ) was subjected to 1,3-bisarylation using 1-methyl indole and $\left(m-\mathrm{ClC}_{6} \mathrm{H}_{4}\right)_{3} \mathrm{BiCl}_{2}$ as arene sources and the crude product was purified via silica gel column chromatography (EtOAc: Hexane 2:8), which afforded compound $5 \mathrm{t}$ in $63 \%$ yield $(0.032 \mathrm{~g})$ as colourless oil. $R_{f} 0.25$ (EtOAc: Hexane 3:7); ${ }^{1} \mathrm{H}$ NMR $\left(\mathrm{CDCl}_{3}, 400 \mathrm{MHz}\right) \delta 7.62$ (s, $1 \mathrm{H}), 7.38(\mathrm{t}, J=7.7 \mathrm{~Hz}, 2 \mathrm{H}), 7.24-7.18(\mathrm{~m}, 4 \mathrm{H}), 7.17-7.13(\mathrm{~m}, 2 \mathrm{H}), 6.99$ (t, J = 7.4 $\mathrm{Hz}, 1 \mathrm{H}), 6.78-6.75(\mathrm{~m}, 3 \mathrm{H}), 4.18(\mathrm{t}, J=6.5 \mathrm{~Hz}, 1 \mathrm{H}), 3.75(\mathrm{~s}, 3 \mathrm{H}), 3.70(\mathrm{~s}, 3 \mathrm{H}), 3.39$ (s, $3 \mathrm{H}), 3.34$ (s, 3H), 3.22 (dd, $J=14.5,5.7 \mathrm{~Hz}, 1 \mathrm{H}), 3.13$ (dd, $J=14.5,7.4 \mathrm{~Hz}, 1 \mathrm{H}$ ); ${ }^{13} \mathrm{C}\left\{{ }^{1} \mathrm{H}\right\}$ NMR $\left(\mathrm{CDCl}_{3}, 100 \mathrm{MHz}\right) \delta 170.5,170.3,158.0,139.0,137.3,136.4,134.2$, $129.4,129.2$, 128.9, 127.9, 126.8, 126.5, 126.4, 121.7, 119.7, 118.9, 118.6, 113.7, 109.2, 61.4, 55.4, 52.7, 52.6, 42.2, 37.9, 32.8; HRMS (ESI-TOF) m/z: [M+Na] ${ }^{+}$ $\mathrm{C}_{29} \mathrm{H}_{28} \mathrm{CINNaO}_{5}$ Calcd. 528.1554, Found 528.1553. 
<smiles>COc1cccc(C([O-])(CC(Cc2ccccc2)(c2ccccc2)c2ccccc2)OC)c1</smiles>

Following the general procedure, D-A cyclopropane $(1 \mathrm{~b}, 0.117 \mathrm{~g}, 0.5 \mathrm{mmol})$ was subjected to 1,3-bisarylation using 1-methylindole and $\left(m-\mathrm{OMeC}_{6} \mathrm{H}_{4}\right)_{3} \mathrm{BiCl}_{2}$ as arene sources and the crude product was purified via silica gel column chromatography (EtOAc: Hexane 2:8), which afforded compound $5 \mathrm{w}$ in $77 \%$ yield $(0.181 \mathrm{~g})$ as colourless oil. $R_{f} 0.35$ (EtOAc: Hexane 3:7); ${ }^{1} \mathrm{H}$ NMR $\left(\mathrm{CDCl}_{3}, 400 \mathrm{MHz}\right) \delta 7.33$ (d, J = $8.0 \mathrm{~Hz}, 1 \mathrm{H}), 7.28-7.22(\mathrm{~m}, 3 \mathrm{H}), 7.21-7.15(\mathrm{~m}, 5 \mathrm{H}), 7.09(\mathrm{~d}, J=8.3 \mathrm{~Hz}, 2 \mathrm{H}), 6.94(\mathrm{t}$, $J=7.3 \mathrm{~Hz}, 1 \mathrm{H}), 6.80(\mathrm{~d}, J=10.2 \mathrm{~Hz}, 1 \mathrm{H}), 6.73(\mathrm{~s}, 1 \mathrm{H}), 4.23(\mathrm{t}, J=6.4 \mathrm{~Hz}, 1 \mathrm{H}), 3.68$ $(\mathrm{s}, 3 \mathrm{H}), 3.63(\mathrm{~s}, 3 \mathrm{H}), 3.31(\mathrm{~s}, 3 \mathrm{H}), 3.24(\mathrm{~s}, 3 \mathrm{H}), 3.21-3.13(\mathrm{~m}, 2 \mathrm{H}) ;{ }^{13} \mathrm{C}\left\{{ }^{1} \mathrm{H}\right\}$ NMR $\left(\mathrm{CDCl}_{3}, 100 \mathrm{MHz}\right) \delta 170.9,170.5,159.4,144.5,138.5,137.2,129.2,128.3,128.2$, $126.9,126.5,126.1,121.6,120.5,119.7,118.8,118.4,114.5,113.1,109.1,61.4$, 55.3, 52.5, 52.3, 42.2, 38.6, 32.7; HRMS (ESI-TOF) m/z: [M+Na] ${ }^{+} \mathrm{C}_{29} \mathrm{H}_{29} \mathrm{NNaO}_{5}$ Calcd. 494.1943, Found 494.1934.

Dimethyl 2-(2,2-bis(4-methoxyphenyl)ethyl)-2-(3-methoxyphenyl)malonate (5x):<smiles>COc1ccc(C(CC(CO)(OC)c2ccc(OC)cc2)c2cccc(OC)c2)cc1</smiles>

This reaction was carried out following the general procedure, except DCM was used as solvent and it was heated at $50{ }^{\circ} \mathrm{C}$ on an oil bath. Accordingly, D-A cyclopropane $(1 \mathrm{a}, 0.027 \mathrm{~g}, 0.1 \mathrm{mmol})$ was subjected to 1,3-bisarylation using anisole and $(\mathrm{m}$ $\left.\mathrm{OMeC}_{6} \mathrm{H}_{4}\right)_{3} \mathrm{BiCl}_{2}$ as arene sources and the crude product was purified via silica gel column chromatography (EtOAc: Hexane 2:8), which afforded compound 5x in 69\% yield $(0.033 \mathrm{~g})$ as colourless oil. $R_{f} 0.35$ (EtOAc: Hexane $\left.3: 7\right) ;{ }^{1} \mathbf{H}$ NMR $\left(\mathrm{CDCl}_{3}, 400\right.$ $\mathrm{MHz}) \delta 7.29(\mathrm{~d}, J=8.7 \mathrm{~Hz}, 1 \mathrm{H}), 7.16-7.14(\mathrm{~m}, 5 \mathrm{H}), 7.09(\mathrm{~d}, J=7.9 \mathrm{~Hz}, 1 \mathrm{H}), 6.87$ (dd, $J=8.1,2.4 \mathrm{~Hz}, 1 \mathrm{H}), 6.81(\mathrm{~d}, J=8.4 \mathrm{~Hz}, 4 \mathrm{H}), 3.95(\mathrm{t}, J=6.1 \mathrm{~Hz}, 1 \mathrm{H}), 3.80$ (s, $3 \mathrm{H}), 3.78(\mathrm{~s}, 6 \mathrm{H}), 3.42(\mathrm{~s}, 6 \mathrm{H}), 3.18(\mathrm{~d}, \mathrm{~J}=6.2 \mathrm{~Hz}, 2 \mathrm{H}) ;{ }^{13} \mathrm{C}\left\{{ }^{1} \mathrm{H}\right\}$ NMR $\left(\mathrm{CDCl}_{3}, 100\right.$ $\mathrm{MHz}) \delta$ 170.7, 159.4, 158.0, 138.3, 137.5, 129.2, 128.8, 120.7, 114.7, 113.9, 113.2, 61.7, 55.4, 55.3, 52.5, 45.6, 41.8; HRMS (ESI-TOF) m/z: $[\mathrm{M}+\mathrm{Na}]{ }^{+} \mathrm{C}_{28} \mathrm{H}_{30} \mathrm{NaO}_{7}$ Calcd. 501.1889, Found 501.1887. 


\section{General procedure for the preparation of compounds 7}

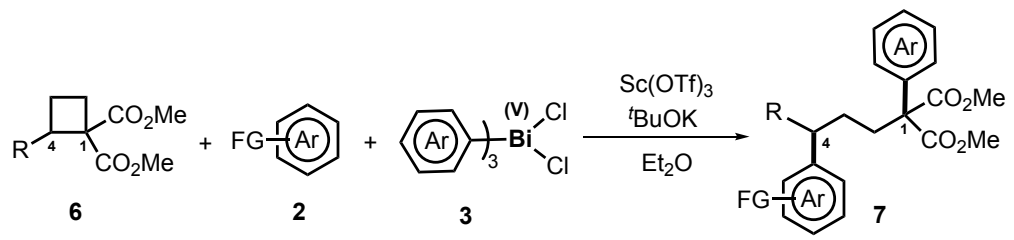

Under $\mathrm{N}_{2}$ atmosphere, donor-Acceptor (D-A) cyclobutane (1.0 equiv), arene (1.5 equiv.) and $\mathrm{Sc}(\mathrm{OTf})_{3}(10 \mathrm{~mol} \%)$ were taken in anhydrous diethyl ether $(0.1 \mathrm{M})$. To this solution were successively added ${ }^{\mathrm{B}} \mathrm{BuOK}$ (2.0 equiv) and $\mathrm{Ar}_{3} \mathrm{BiCl}_{2}$ (1.25 equiv) in one portion and the reaction mixture was stirred at room temperature until completion of the reaction as determined by TLC analysis (ca. 4-10 h). When the starting material was consumed, the crude production was purified by column chromatography on silica gel to provide the pure product. 


\title{
8. Characterization data of compounds 7
}

\author{
Dimethyl 2-(3-(1,3-dimethyl-1H-indol-2-yl)-3-(4-methoxyphenyl)propyl)-2- \\ phenylmalonate (7a):
}<smiles>COc1ccc(C(CCC(OC)(OC)C(C)(O)c2ccccc2)c2c(C)c3ccccc3n2C)cc1</smiles>

Following the general procedure, D-A cyclobutane $(6 \mathbf{a}, 0.028 \mathrm{~g}, 0.1 \mathrm{mmol})$ was subjected to 1,3-bisarylation using 1,3-dimethyl indole and $\mathrm{Ph}_{3} \mathrm{BiCl}_{2}$ as arene sources and the crude product was purified via silica gel column chromatography (EtOAc: Hexane 1:9), which afforded compound $7 \mathrm{a}$ in $78 \%$ yield $(0.039 \mathrm{~g})$ as colourless oil. $R_{f} 0.35$ (EtOAc: Hexane 3:7); FT-IR $\left(\mathrm{v} \mathrm{cm}^{-1}\right): 2951,1734,1609,1510$, 1247; ${ }^{1} \mathrm{H}$ NMR $\left(\mathrm{CDCl}_{3}, 400 \mathrm{MHz}\right) \delta 7.55(\mathrm{~d}, J=7.8 \mathrm{~Hz}, 1 \mathrm{H}), 7.25-7.20(\mathrm{~m}, 5 \mathrm{H})$, 7.18-7.14 (m, 2H), $7.11-7.07(\mathrm{~m}, 1 \mathrm{H}), 7.03(\mathrm{~d}, J=8.4 \mathrm{~Hz}, 2 \mathrm{H}), 6.75(\mathrm{~d}, J=8.4 \mathrm{~Hz}$, $2 \mathrm{H}), 4.34(\mathrm{dd}, J=11.2,4.2 \mathrm{~Hz}, 1 \mathrm{H}), 3.74(\mathrm{~s}, 3 \mathrm{H}), 3.72(\mathrm{~s}, 3 \mathrm{H}), 3.67(\mathrm{~s}, 3 \mathrm{H}), 3.31(\mathrm{~s}$, $3 \mathrm{H}), 2.41-2.35(\mathrm{~m}, 1 \mathrm{H}), 2.30(\mathrm{~s}, 3 \mathrm{H}), 2.25-2.20(\mathrm{~m}, 2 \mathrm{H}), 2.06-1.98(\mathrm{~m}, 1 \mathrm{H}) ;{ }^{13} \mathrm{C}$ $\left\{{ }^{1} \mathrm{H}\right\}$ NMR $\left(\mathrm{CDCl}_{3}, 100 \mathrm{MHz}\right) \delta 171.2(2), 158.0,137.1,137.0,136.8,134.2,128.6$, $128.5,128.4,128.0,127.8,121.1,118.7,118.4,113.8,108.7,108.5,62.65,55.4$, 52.9 (2), 40.6, 34.2, 30.5, 28.1, 9.5; HRMS (ESI-TOF) m/z: $[\mathrm{M}+\mathrm{Na}]^{+} \mathrm{C}_{31} \mathrm{H}_{33} \mathrm{NNaO}_{5}$ Calcd. 522.2256, Found 522.2252.

Dimethyl 2-(3-(4-(benzyloxy)phenyl)-3-(1,3-dimethyl-1H-indol-2-yl)propyl)-2phenylmalonate $(\mathbf{7 b})$ :<smiles>COC(=O)c1ccc(Oc2ccccc2)cc1C(CCC(C)(OC)C(=O)c1ccccc1)c1c(C)c2ccccc2n1C</smiles>

Following the general procedure, D-A cyclobutane $(6 \mathrm{~b}, 0.035 \mathrm{~g}, 0.1 \mathrm{mmol})$ was subjected to 1,3-bisarylation using 1,3-dimethyl indole and $\mathrm{Ph}_{3} \mathrm{BiCl}_{2}$ as arene sources and the crude product was purified via silica gel column chromatography (EtOAc: Hexane 1:9), which afforded compound $7 \mathbf{b}$ in $75 \%$ yield $(0.043 \mathrm{~g})$ as colourless oil. $R_{f} 0.35$ (EtOAc: Hexane 3:7); ${ }^{1} \mathrm{H}$ NMR $\left(\mathrm{CDCl}_{3}, 400 \mathrm{MHz}\right) \delta 7.58(\mathrm{~d}, \mathrm{~J}=$ $7.7 \mathrm{~Hz}, 1 \mathrm{H}), 7.43-7.32(\mathrm{~m}, 5 \mathrm{H}), 7.29-7.25(\mathrm{~m}, 5 \mathrm{H}), 7.20(\mathrm{~d}, J=5.7 \mathrm{~Hz}, 2 \mathrm{H}), 7.14-$ $7.11(\mathrm{~m}, 1 \mathrm{H}), 7.06(\mathrm{~d}, J=8.5 \mathrm{~Hz}, 2 \mathrm{H}), 6.86(\mathrm{~d}, J=8.4 \mathrm{~Hz}, 2 \mathrm{H}), 5.02(\mathrm{~s}, 2 \mathrm{H}), 4.37$ (dd, $J=10.5,4.2 \mathrm{~Hz}, 1 \mathrm{H}), 3.75(\mathrm{~s}, 3 \mathrm{H}), 3.70(\mathrm{~s}, 3 \mathrm{H}), 3.35(\mathrm{~s}, 3 \mathrm{H}), 2.44-2.36(\mathrm{~m}$, $1 \mathrm{H}), 2.33(\mathrm{~s}, 3 \mathrm{H}), 2.26-2.24(\mathrm{~m}, 2 \mathrm{H}), 2.10-2.02(\mathrm{~m}, 1 \mathrm{H}) ;{ }^{13} \mathrm{C}\left\{{ }^{1} \mathrm{H}\right\}$ NMR $\left(\mathrm{CDCl}_{3}, 100\right.$ $\mathrm{MHz}) \delta 171.3,171.2,157.3,137.1,137.1,137.0,136.9,134.6,128.7,128.6,128.6$, $128.4,128.1,128.0,127.8,127.6,121.1,118.7,118.4,114.9,108.7,108.5,70.2$, $62.7,52.8,52.9,40.7,34.3,30.5,28.2,9.5$; HRMS (ESI-TOF) $\mathrm{m} / \mathrm{z}:[\mathrm{M}+\mathrm{Na}]^{+}$ $\mathrm{C}_{37} \mathrm{H}_{37} \mathrm{NNaO}_{5}$ Calcd. 598.2569, Found 598.2567. 
Dimethyl 2-(3-(1,3-dimethyl-1H-indol-2-yl)-3-(4-methoxyphenyl)propyl)-2-(mtolyl)malonate (7c):<smiles>COc1ccc(C(CCC(C)(OC)c2cccc(C)c2)c2c(C)c3ccccc3n2C)cc1</smiles>

Following the general procedure, D-A cyclobutane $(6 \mathrm{a}, 0.028 \mathrm{~g}, 0.1 \mathrm{mmol})$ was subjected to 1,3-bisarylation using 1,3-dimethyl indole and $\left(m-\mathrm{MeC}_{6} \mathrm{H}_{4}\right)_{3} \mathrm{BiCl}_{2}$ as arene sources and the crude product was purified via silica gel column chromatography (EtOAc: Hexane 1:9), which afforded compound 7c in $63 \%$ yield $(0.032 \mathrm{~g})$ as colourless oil. $R_{f} 0.35$ (EtOAc: Hexane 2:8); ${ }^{1} \mathrm{H}$ NMR $\left(\mathrm{CDCl}_{3}, 400 \mathrm{MHz}\right)$ $\delta 7.56(\mathrm{~d}, J=7.8 \mathrm{~Hz}, 1 \mathrm{H}), 7.18(\mathrm{~d}, J=5.5 \mathrm{~Hz}, 2 \mathrm{H}), 7.16-7.09(\mathrm{~m}, 3 \mathrm{H}), 7.07-7.04$ $(\mathrm{m}, 4 \mathrm{H}), 6.77(\mathrm{~d}, J=8.3 \mathrm{~Hz}, 2 \mathrm{H}), 4.36(\mathrm{dd}, J=10.6,4.4 \mathrm{~Hz}, 1 \mathrm{H}), 3.76(\mathrm{~s}, 3 \mathrm{H}), 3.74$ (s, 3H), $3.69(\mathrm{~s}, 3 \mathrm{H}), 3.35(\mathrm{~s}, 3 \mathrm{H}), 2.43-2.35(\mathrm{~m}, 1 \mathrm{H}), 2.31(\mathrm{~s}, 3 \mathrm{H}), 2.28(\mathrm{~s}, 3 \mathrm{H}), 2.25$ - $2.16(\mathrm{~m}, 2 \mathrm{H}), 2.09-2.01(\mathrm{~m}, 1 \mathrm{H}) ;{ }^{13} \mathrm{C}\left\{{ }^{1} \mathrm{H}\right\}$ NMR $\left(\mathrm{CDCl}_{3}, 100 \mathrm{MHz}\right) \delta 171.4,171.3$, $158.1,138.0,137.1,137.1,136.8,134.3,128.6,128.6,128.6,128.5,128.3,125.1$, 121.1, 118.7, 118.4, 113.9, 108.7, 108.4, 62.7, 55.4, 52.9, 52.8, 40.7, 34.4, 30.5, 28.2, 21.8, 9.5; HRMS (ESI-TOF) m/z: $[\mathrm{M}+\mathrm{Na}]^{+} \mathrm{C}_{32} \mathrm{H}_{35} \mathrm{NNaO}_{5}$ Calcd. 536.2413, Found 536.2385.

\section{Dimethyl 2-(3-(1,3-dimethyl-1H-indol-2-yl)-3-(4-methoxyphenyl)propyl)-2-(3- methoxyphenyl)malonate $(7 \mathrm{~d})$ :}

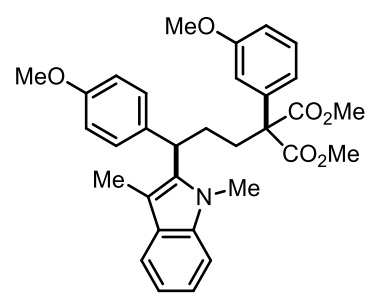

Following the general procedure, D-A cyclobutane $(6 \mathbf{a}, 0.028 \mathrm{~g}, 0.1 \mathrm{mmol})$ was subjected to 1,3-bisarylation using 1,3-dimethyl indole and $\left(m-\mathrm{OMeC}_{6} \mathrm{H}_{4}\right)_{3} \mathrm{BiCl}_{2}$ as arene sources and the crude product was purified via silica gel column chromatography (EtOAc: Hexane 2:8), which afforded compound $\mathbf{7 d}$ in $59 \%$ yield $(0.031 \mathrm{~g})$ as colourless oil. $R_{f} 0.20$ (EtOAc: Hexane 2:8); ${ }^{1} \mathrm{H}$ NMR $\left(\mathrm{CDCl}_{3}, 400 \mathrm{MHz}\right)$ $\delta 7.55(\mathrm{~d}, J=7.7 \mathrm{~Hz}, 1 \mathrm{H}), 7.20-7.15(\mathrm{~m}, 3 \mathrm{H}), 7.12-7.09(\mathrm{~m}, 1 \mathrm{H}), 7.05(\mathrm{~d}, J=8.4$ $\mathrm{Hz}, 2 \mathrm{H}), 6.86(\mathrm{~s}, 1 \mathrm{H}), 6.83-6.80(\mathrm{~m}, 2 \mathrm{H}), 6.77(\mathrm{~d}, J=8.4 \mathrm{~Hz}, 2 \mathrm{H}), 4.35$ (dd, $J=$ $10.2,4.9 \mathrm{~Hz}, 1 \mathrm{H}), 3.76(\mathrm{~s}, 3 \mathrm{H}), 3.73(\mathrm{~s}, 3 \mathrm{H}), 3.72(\mathrm{~s}, 3 \mathrm{H}), 3.69(\mathrm{~s}, 3 \mathrm{H}), 3.34(\mathrm{~s}, 3 \mathrm{H})$, 2.42 - $2.36(\mathrm{~m}, 1 \mathrm{H}), 2.31(\mathrm{~s}, 3 \mathrm{H}), 2.27-2.17(\mathrm{~m}, 2 \mathrm{H}), 2.09-2.01(\mathrm{~m}, 1 \mathrm{H}) ;{ }^{13} \mathrm{C}\left\{{ }^{1} \mathrm{H}\right\}$ NMR $\left(\mathrm{CDCl}_{3}, 100 \mathrm{MHz}\right) \delta 171.1$ (2), 159.5, 158.1, 138.4, 137.1, 137.0, 134.3, 129.3, 128.6, 121.1, 120.2, 118.7, 118.4, 114.4, 113.9, 112.9, 108.7, 108.4, 62.6, 55.4, 55.3, 52.9, 52.8, 40.6, 34.6, 30.5, 28.2, 9.5; HRMS (ESI-TOF) $\mathrm{m} / \mathrm{z}:[\mathrm{M}+\mathrm{Na}]^{+}$ $\mathrm{C}_{32} \mathrm{H}_{35} \mathrm{NNaO}_{6}$ Calcd. 552.2362, Found 552.2352. 
<smiles>COc1ccc(C(CCC(C)(OC)C(C)=O)c2c(C)c3ccccc3n2C)cc1</smiles>

Following the general procedure, D-A cyclobutane $(1 \mathrm{a}, 0.028 \mathrm{~g}, 0.1 \mathrm{mmol})$ was subjected to 1,3-bisarylation using 1,3-dimethyl indole and $\left(p-\mathrm{FC}_{6} \mathrm{H}_{4}\right)_{3} \mathrm{BiCl}_{2}$ as arene sources and the crude product was purified via silica gel column chromatography (EtOAc: Hexane 1:9), which afforded compound $7 \mathrm{e}$ in $62 \%$ yield $(0.032 \mathrm{~g})$ as colourless oil. $R_{f} 0.35$ (EtOAc: Hexane 3:7); FT-IR $\left(\mathrm{v} \mathrm{cm}^{-1}\right)$ : 2950, 1735, 1608, 1512, 1248; ${ }^{1} \mathrm{H}$ NMR $\left(\mathrm{CDCl}_{3}, 400 \mathrm{MHz}\right) \delta 7.58(\mathrm{~d}, \mathrm{~J}=7.7 \mathrm{~Hz}, 1 \mathrm{H}), 7.24-7.18(\mathrm{~m}, 4 \mathrm{H}), 7.15$ - $7.11(\mathrm{~m}, 1 \mathrm{H}), 7.07(\mathrm{~d}, J=8.5 \mathrm{~Hz}, 2 \mathrm{H}), 6.95(\mathrm{t}, J=8.6 \mathrm{~Hz}, 2 \mathrm{H}), 6.79(\mathrm{~d}, J=8.5 \mathrm{~Hz}$, $2 \mathrm{H}), 4.37(\mathrm{dd}, J=10.4,4.3 \mathrm{~Hz}, 1 \mathrm{H}), 3.77(\mathrm{~s}, 3 \mathrm{H}), 3.75(\mathrm{~s}, 3 \mathrm{H}), 3.69(\mathrm{~s}, 3 \mathrm{H}), 3.35(\mathrm{~s}$, $3 \mathrm{H}), 2.43-2.37(\mathrm{~m}, 1 \mathrm{H}), 2.33(\mathrm{~s}, 3 \mathrm{H}), 2.25-2.19(\mathrm{~m}, 2 \mathrm{H}), 2.07-1.98(\mathrm{~m}, 1 \mathrm{H}) ;{ }^{13} \mathrm{C}$ $\left\{{ }^{1} \mathrm{H}\right\}$ NMR $\left(\mathrm{CDCl}_{3}, 100 \mathrm{MHz}\right) \delta 171.1,162.1(\mathrm{~d}, J=246 \mathrm{~Hz}), 158.1,137.1,136.87$, 134.1, 132.4(d, J=3.6) 129.9(d, J=8 Hz) 128.6, 121.2, 118.8, 118.4, 115.2(d, J=21.3 $\mathrm{Hz}) 113.9,108.7,108.5,62.0,55.4,52.9,40.5,34.1,30.5,28.1$, 9.5; HRMS (ESITOF) $\mathrm{m} / \mathrm{z}:[\mathrm{M}+\mathrm{Na}]^{+} \mathrm{C}_{31} \mathrm{H}_{32} \mathrm{FNNaO}_{5}$ Calcd. 540.2162, Found 540.2153.

\section{Dimethyl phenylmalonate (7f) \\ 2-(3-(4-methoxyphenyl)-3-(1-methyl-1H-indol-3-yl)propyl)-2-}<smiles>COC(CCc1ccccc1)(c1ccccc1)c1ccc(C)cc1</smiles>

Following the general procedure, D-A cyclobutane $(6 \mathbf{a}, 0.028 \mathrm{~g}, 0.1 \mathrm{mmol})$ was subjected to 1,3-bisarylation using 1-methyl indole and $\mathrm{Ph}_{3} \mathrm{BiCl}_{2}$ as arene sources and the crude product was purified via silica gel column chromatography (EtOAc: Hexane 1:9), which afforded compound $7 \mathrm{f}$ in $76 \%$ yield $(0.037 \mathrm{~g})$ as colourless oil. $R_{f}$ 0.35 (EtOAc: Hexane 3:7); ${ }^{1} \mathrm{H}$ NMR $\left(\mathrm{CDCl}_{3}, 400 \mathrm{MHz}\right): \delta 7.40(\mathrm{~d}, J=7.9 \mathrm{~Hz}, 1 \mathrm{H})$, $7.33-7.24(\mathrm{~m}, 6 \mathrm{H}), 7.21-7.15(\mathrm{~m}, 3 \mathrm{H}), 7.00(\mathrm{t}, J=7.5 \mathrm{~Hz}, 1 \mathrm{H}), 6.81(\mathrm{~d}, J=8.4 \mathrm{~Hz}$, $2 \mathrm{H}), 6.76(\mathrm{~s}, 1 \mathrm{H}), 4.10(\mathrm{t}, J=7.5 \mathrm{~Hz}, 1 \mathrm{H}), 3.77(\mathrm{~s}, 3 \mathrm{H}), 3.72(\mathrm{~s}, 9 \mathrm{H}), 2.47-2.30(\mathrm{~m}$, $2 \mathrm{H}), 2.18-2.09(\mathrm{~m}, 1 \mathrm{H}), 1.97-1.89(\mathrm{~m}, 1 \mathrm{H}) ;{ }^{13} \mathrm{C}\left\{{ }^{1} \mathrm{H}\right\}$ NMR $\left(\mathrm{CDCl}_{3}, 100 \mathrm{MHz}\right): \delta$ $171.4,171.3,158.0,137.3,137.1,137.0,128.9,128.3,128.1,127.7,127.4,126.0$, 121.6, 119.6, 118.8, 118.7, 113.8, 109.2, 62.8, 55.3, 52.8, 42.4, 34.4, 32.8, 31.3; HRMS (ESI-TOF) m/z: $[\mathrm{M}+\mathrm{Na}]^{+} \mathrm{C}_{30} \mathrm{H}_{31} \mathrm{NNaO}_{5}$ Calcd. 508.2100, Found 508.2091. 

phenylmalonate $(7 \mathrm{~g})$<smiles>COc1ccc(C(CCC(C)(OC)c2ccccc2)c2c([N+](=O)[O-])n(C)c3ccccc23)cc1</smiles>

Following the general procedure, D-A cyclobutane $(6 \mathrm{a}, 0.028 \mathrm{~g}, 0.1 \mathrm{mmol})$ was subjected to 1,3-bisarylation using 1,2-dimethyl indole and $\mathrm{Ph}_{3} \mathrm{BiCl}_{2}$ as arene sources and the crude product was purified via silica gel column chromatography (EtOAc: Hexane 1:9), which afforded compound $7 \mathrm{~g}$ in $70 \%$ yield $(0.033 \mathrm{~g})$ as colourless oil. $R_{f} 0.35$ (EtOAc: Hexane 3:7); ${ }^{1} \mathbf{H}$ NMR $\left(\mathrm{CDCl}_{3}, 400 \mathrm{MHz}\right): \delta 7.40$ (d, J $=8.0 \mathrm{~Hz}, 1 \mathrm{H}), 7.32-7.28(\mathrm{~m}, 6 \mathrm{H}), 7.23-7.20(\mathrm{~m}, 2 \mathrm{H}), 7.14(\mathrm{t}, J=7.5 \mathrm{~Hz}, 1 \mathrm{H}), 6.98$ $(\mathrm{t}, J=7.5 \mathrm{~Hz}, 1 \mathrm{H}), 6.80-6.76(\mathrm{~m}, 2 \mathrm{H}), 4.17(\mathrm{t}, J=7.5 \mathrm{~Hz}, 1 \mathrm{H}), 3.77(\mathrm{~s}, 3 \mathrm{H}), 3.71(\mathrm{~s}$, $3 \mathrm{H}), 3.70(\mathrm{~s}, 3 \mathrm{H}), 3.64(\mathrm{~s}, 3 \mathrm{H}), 2.44-2.33(\mathrm{~m}, 4 \mathrm{H}), 2.30-2.17(\mathrm{~m}, 3 \mathrm{H}) ;{ }^{13} \mathrm{C}\left\{{ }^{1} \mathrm{H}\right\}$ NMR $\left(\mathrm{CDCl}_{3}, 100 \mathrm{MHz}\right): \delta 171.5,171.3,157.6,137.5,137.0,136.7,133.4,128.6$, 128.3, 128.2, 127.6, 126.7, 120.4, 119.6, 118.7, 113.6, 113.1, 108.7, 62.7, 55.3, 52.7, 52.7, 41.8, 34.4, 29.7, 29.7, 10.8; HRMS (ESI-TOF) m/z: $[\mathrm{M}+\mathrm{Na}]^{+}$ $\mathrm{C}_{31} \mathrm{H}_{33} \mathrm{NNaO}_{5}$ Calcd. 522.2256, Found 522.2250.

Dimethyl 2-(3-(4-(benzyloxy)phenyl)-3-(2,4,6-trimethoxyphenyl)propyl)-2-(3methoxyphenyl)malonate $(7 \mathrm{~h})$ :<smiles></smiles>

This reaction was carried out following the general procedure, except DCM was used as solvent and it was heated at $50{ }^{\circ} \mathrm{C}$ on an oil bath. Accordingly, D-A cyclobutane $(6 \mathrm{~b}, 0.035 \mathrm{~g}, 0.1 \mathrm{mmol})$ was subjected to 1,3-bisarylation using 1, 3, 5trimethxoybenzene and $\left(m-\mathrm{OMeC}_{6} \mathrm{H}_{4}\right)_{3} \mathrm{BiCl}_{2}$ as arene sources and the crude product was purified via silica gel column chromatography (EtOAc: Hexane 2:8), which afforded compound $7 \mathrm{~h}$ in $81 \%$ yield $(0.051 \mathrm{~g})$ as colourless oil. $R_{f} 0.20$ (EtOAc: Hexane 2:8); ${ }^{1} \mathrm{H}$ NMR $\left(\mathrm{CDCl}_{3}, 400 \mathrm{MHz}\right) \delta 7.41(\mathrm{~d}, \mathrm{~J}=7.5 \mathrm{~Hz}, 2 \mathrm{H}), 7.36(\mathrm{t}, \mathrm{J}=7.4$ $\mathrm{Hz}, 2 \mathrm{H}), 7.30(\mathrm{t}, J=7.1 \mathrm{~Hz}, 1 \mathrm{H}), 7.22(\mathrm{t}, J=8.0 \mathrm{~Hz}, 1 \mathrm{H}), 7.16(\mathrm{~d}, J=8.4 \mathrm{~Hz}, 2 \mathrm{H})$, $6.98(\mathrm{~s}, 1 \mathrm{H}), 6.95(\mathrm{~d}, J=7.9 \mathrm{~Hz}, 1 \mathrm{H}), 6.81(\mathrm{t}, J=6.8 \mathrm{~Hz}, 3 \mathrm{H}), 6.10(\mathrm{~s}, 2 \mathrm{H}), 5.00(\mathrm{~s}$, $2 \mathrm{H}), 4.52(\mathrm{dd}, J=9.1,6.1 \mathrm{~Hz}, 1 \mathrm{H}), 3.78(\mathrm{~s}, 6 \mathrm{H}), 3.72(\mathrm{~s}, 3 \mathrm{H}), 3.69(\mathrm{~s}, 9 \mathrm{H}), 2.35-2.27$ $(\mathrm{m}, 1 \mathrm{H}), 2.24-2.10(\mathrm{~m}, 2 \mathrm{H}), 2.04-1.95(\mathrm{~m}, 1 \mathrm{H}) ;{ }^{13} \mathrm{C}\left\{{ }^{1} \mathrm{H}\right\}$ NMR $\left(\mathrm{CDCl}_{3}, 100 \mathrm{MHz}\right) \delta$ $171.3,171.3,159.6,159.4,159.2$, 156.6, 138.6, 137.9, 137.6, 129.0, 128.9, 128.6, $127.9,127.6,120.6,114.7,114.1,113.4,112.7,91.3,70.1,62.7,55.7,55.3,55.3$, 52.7, 52.6, 38.8, 34.6, 27.1; HRMS (ESI-TOF) m/z: [M+Na] ${ }^{+} \mathrm{C}_{37} \mathrm{H}_{40} \mathrm{NaO}_{9}$ Calcd. 651.2570, Found 651.2568. 


\section{Synthesis of highly branched $\alpha$-aryl carboxylic acid derivatives from 1,3- diarylated products $(8 \mathrm{a}-8 \mathrm{c})$}

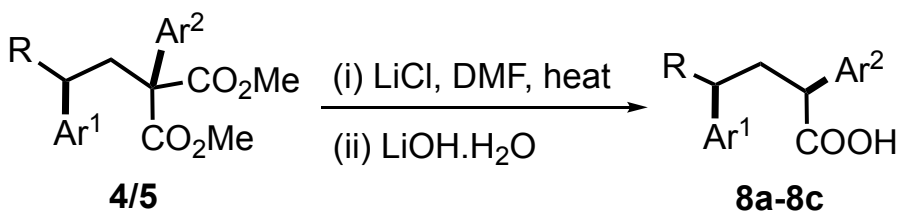

In a $10 \mathrm{~mL}$ sealed tube were successively added compound $\mathbf{4 / 5}(0.5 \mathrm{mmol}, 1$ equiv), $\mathrm{LiCl}(1.0 \mathrm{mmol}, 2.0$ equiv), water $(60.0 \mu \mathrm{L})$ and DMF $(5.0 \mathrm{~mL})$. The reaction mixture was heated to reflux for $8 \mathrm{~h}$ on an oil bath. After completion, the mixture was cooled and diluted with $\mathrm{CH}_{2} \mathrm{Cl}_{2}(10 \mathrm{~mL})$. The combined organic layer was washed with water and extracted in $\mathrm{CH}_{2} \mathrm{Cl}_{2}(3 \times 5 \mathrm{~mL})$. The combined organic layer was collected, dried and evaporated under reduced pressure. The crude residue was directly used in the next step without further purification. The crude was taken into THF $(5.0 \mathrm{~mL})$ and was added a solution of $\mathrm{LiOH} . \mathrm{H}_{2} \mathrm{O}(1.5 \mathrm{mmol})$ in $\mathrm{MeOH}-\mathrm{H}_{2} \mathrm{O}(4: 1,5.0 \mathrm{~mL})$ drop wise. The resulting mixture was stirred at room temperature for $6 \mathrm{~h}$. It was then quenched with $10 \%$ aqueous $\mathrm{HCl}(\mathrm{pH} \sim 2)$ and the solvent was removed under reduced pressure. The residue was further diluted with ethyl acetate $(10 \mathrm{~mL})$ and washed with water $(2 \times 5 \mathrm{~mL})$. The combined organic layer was dried over anhydrous $\mathrm{Na}_{2} \mathrm{SO}_{4}$, filtered and concentrated under reduced pressure, and finally purified through silica gel column chromatography EtOAc/ PE (3:7) to afford the final products. A baseline tail was observed in TLC analysis (EtOAc: Hexane 3:7).

\section{4-(4-methoxyphenyl)-4-(1-methyl-1H-indol-3-yl)-2-phenylbutanoic acid (8a):}<smiles>COc1ccc(C(CC(CO)c2ccccc2)c2cn(C)c3ccccc23)cc1</smiles>

Following the general procedure, compound $4 \mathrm{a}(0.24 \mathrm{~g}, 0.5 \mathrm{mmol})$ was transformed into the a-aryl carboxylic acid derivative $8 \mathrm{a}$ in $78 \%$ yield $(0.155 \mathrm{~g}$, colorless oil) as inseparable mixture of diastereomers $(d r 2: 1)$. ${ }^{1} \mathbf{H} \mathbf{N M R}\left(\mathrm{CDCl}_{3}, 400 \mathrm{MHz}\right) \delta 7.39(\mathrm{~d}, J$ $=8.0 \mathrm{~Hz}, 1 \mathrm{H}), 7.29(\mathrm{~d}, J=7.2 \mathrm{~Hz}, 1 \mathrm{H}), 7.23(\mathrm{dd}, J=7.5,2.0 \mathrm{~Hz}, 3 \mathrm{H}), 7.20-7.16(\mathrm{~m}$, $5 \mathrm{H}), 7.14-7.06(\mathrm{~m}, 4 \mathrm{H}), 6.96(\mathrm{t}, J=7.4 \mathrm{~Hz}, 1 \mathrm{H}), 6.89(\mathrm{t}, J=7.6 \mathrm{~Hz}, 0.5 \mathrm{H}) 6.78(\mathrm{~s}$, $1 \mathrm{H}), 6.75-6.73(\mathrm{~m}, 3 \mathrm{H}), 4.02(\mathrm{t}, J=7.7 \mathrm{~Hz}, 1 \mathrm{H}), 3.88(\mathrm{t}, J=7.7 \mathrm{~Hz}, 0.5 \mathrm{H}) 3.70(\mathrm{~s}$, $4 \mathrm{H}), 3.66(\mathrm{~s}, 3 \mathrm{H}), 3.65(\mathrm{~s}, 1.5 \mathrm{H}), 3.51(\mathrm{t}, J=7.4 \mathrm{~Hz}, 1 \mathrm{H}), 3.46(\mathrm{t} J=8.0 \mathrm{~Hz}, 0.5 \mathrm{H}) 2.99$ - $2.92(\mathrm{~m}, 1 \mathrm{H}), 2.69-2.64(\mathrm{~m}, 1 \mathrm{H}), 2.34-2.27(\mathrm{~m}, 1 \mathrm{H}) ;{ }^{13} \mathrm{C}\left\{{ }^{1} \mathrm{H}\right\}$ NMR $\left(\mathrm{CDCl}_{3}, 100\right.$ $\mathrm{MHz}) \delta 179.7,158.2$ 158.1, 138.8, 138.1, 137.4, 136.5, 135.9, 129.1, 128.9 (2), $128.8,128.6,128.3,127.8,127.6,127.4,127.2,126.1,125.9,121.8,121.7,119.8$, 119.6, 118.9, 118.8, 118.0, 114.0 (2), 109.24, 55.36, 49.55, 49.33, 39.8, 39.3, 39.0, 32.8(2); HRMS (ESI-TOF) m/z: [M+Na] ${ }^{+} \quad \mathrm{C}_{26} \mathrm{H}_{25} \mathrm{NNaO}_{3}$ Calcd. 422.1732, Found 422.1725. 
<smiles>CN(C)c1ccccc1C(c1ccccc1)C(Cc1ccccc1)C(=O)O</smiles>

Following the general procedure, compound $4 \mathrm{t}(0.224 \mathrm{~g}, 0.5 \mathrm{mmol})$ was transformed into the $\alpha$-aryl carboxylic acid derivative $8 \mathrm{~b}$ in $71 \%$ yield $(0.13 \mathrm{~g}$, colorless oil) as inseparable mixture of diastereomers $\left(d r\right.$ 1:1). FT-IR $\left(\mathrm{v} \mathrm{cm}^{-1}\right): 3028,2929,1703$, 1473, 742; ${ }^{1} \mathrm{H}$ NMR $\left(\mathrm{CDCl}_{3}, 400 \mathrm{MHz}\right) \delta 7.39(\mathrm{~d}, \mathrm{~J}=7.9 \mathrm{~Hz}, 1 \mathrm{H}), 7.24-7.17(\mathrm{~m}, 7 \mathrm{H})$, $7.14(\mathrm{dd}, J=7.7,2.7 \mathrm{~Hz}, 4 \mathrm{H}), 7.10-7.04(\mathrm{~m}, 2 \mathrm{H}), 7.02-6.99(\mathrm{~m}, 2 \mathrm{H}), 6.94(\mathrm{t}, \mathrm{J}=$ $7.4 \mathrm{~Hz}, 1 \mathrm{H}), 6.88(\mathrm{t}, \mathrm{J}=7.4 \mathrm{~Hz}, 1 \mathrm{H}), 6.81-6.78(\mathrm{~m}, 4 \mathrm{H}), 6.73(\mathrm{~s}, 1 \mathrm{H}), 4.27-4.16(\mathrm{~m}$, $2 \mathrm{H}), 3.60(\mathrm{~s}, 3 \mathrm{H}), 3.59(\mathrm{~s}, 2.4 \mathrm{H}), 3.50(\mathrm{t}, J=7.5 \mathrm{~Hz}, 1.8 \mathrm{H}), 2.96-2.89(\mathrm{~m}, 1 \mathrm{H}), 2.77-$ $2.69(\mathrm{~m}, 0.8 \mathrm{H}), 2.63-2.56(\mathrm{~m}, 0.8 \mathrm{H}), 2.46-2.39(\mathrm{~m}, 1 \mathrm{H}) ;{ }^{13} \mathrm{C}\left\{{ }^{1} \mathrm{H}\right\}$ NMR $\left(\mathrm{CDCl}_{3}, 100\right.$ $\mathrm{MHz}) \delta 180.1$ (2), 149.1, 148.4, 138.2, 137.9, 137.3 (2), 128.9 (2), 128.5, 128.4, 127.8 (2), 126.8, 126.7, 126.6, 126.5, 126.3, 124.5, 124.2, 123.8, 123.6, 121.8 (2), 119.7, 119.4, 119.1, 119.0, 117.6, 116.9, 109.4 (2), 49.5, 49.4, 40.3, 40.1, 35.9, 35.62 32.9, 32.8; HRMS (ESI-TOF) m/z: [M+Na] ${ }^{+} \mathrm{C}_{23} \mathrm{H}_{21} \mathrm{NNaO}_{2} \mathrm{~S}$ Calcd. 398.1191, Found 398.1184.

\section{4-(4-methoxyphenyl)-2-phenyl-4-(2,4,6-trimethoxyphenyl)butanoic acid (8c):}

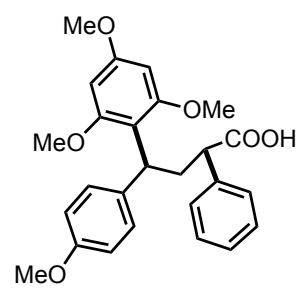

Following the general procedure, compound $\mathbf{5 g}(0.254 \mathrm{~g}, 0.5 \mathrm{mmol})$ was transformed into the a-aryl carboxylic acid derivative $8 \mathrm{c}$ in $73 \%$ yield $(0.16 \mathrm{~g}$, colorless oil) as inseparable mixture of diastereomers $(d r 1: 1) .{ }^{1} \mathbf{H}$ NMR $\left(\mathrm{CDCl}_{3}, 400 \mathrm{MHz}\right) \delta 7.24-$ $7.19(\mathrm{~m}, 7 \mathrm{H}), 7.17-7.13(\mathrm{~m}, 6 \mathrm{H}), 6.70(\mathrm{t}, J=8.1 \mathrm{~Hz}, 4 \mathrm{H}), 6.04(\mathrm{~s}, 4 \mathrm{H}), 4.61(\mathrm{dd}, J=$ 10.6, $5.8 \mathrm{~Hz}, 0.8 \mathrm{H}), 4.28$ (dd, J = 11.0, $5.5 \mathrm{~Hz}, 1 \mathrm{H}), 3.74(\mathrm{~s}, 2 \mathrm{H}), 3.72(\mathrm{~s}, 3 \mathrm{H}), 3.70$ (s, 2H), $3.69(\mathrm{~s}, 3 \mathrm{H}), 3.58(\mathrm{~s}, 6 \mathrm{H}), 3.55(\mathrm{~s}, 4 \mathrm{H}), 3.34(\mathrm{dd}, J=9.9,5.2 \mathrm{~Hz}, 2 \mathrm{H}), 3.16$ $3.09(\mathrm{~m}, 1 \mathrm{H}), 2.83-2.76(\mathrm{~m}, 0.8 \mathrm{H}), 2.63-2.56(\mathrm{~m}, 0.8 \mathrm{H}), 2.53-2.46(\mathrm{~m}, 1 \mathrm{H}) ;{ }^{13} \mathrm{C}$ $\left\{{ }^{1} \mathrm{H}\right\}$ NMR $\left(\mathrm{CDCl}_{3}, 100 \mathrm{MHz}\right) \delta 180.8,180.6,159.9,159.8,159.3,157.4,157.3$, $139.5,138.5,137.2,137.0,128.8$ (2), 128.7, 128.6, 128.4, 128.1, 127.3, 113.2, 113.1, 112.4, 111.7, 91.2, 91.0, 55.5, 55.3 (2), 50.2, 50.1, 36.3, 36.1, 36.0, 35.4; HRMS (ESI-TOF) m/z: [M+Na] ${ }^{+} \mathrm{C}_{26} \mathrm{H}_{28} \mathrm{NaO}_{6}$ Calcd. 459.1784, Found 459.1778. 


\section{Synthesis of tri-substituted olefins (9)}

In a $10 \mathrm{~mL}$ sealed tube, DDQ $(0.12 \mathrm{mmol}, 1.2$ equiv. $)$ was added to a solution of the 1,3- or 1,4-bisarylated compounds (4 or $5,0.1 \mathrm{mmol}, 1.0$ equiv) in dry $\mathrm{CH}_{2} \mathrm{Cl}_{2}(1.0$ $\mathrm{mL}$ ) at $0{ }^{\circ} \mathrm{C}$. The mixture was stirred at same temperature for $1 \mathrm{~h}$. Upon completion of the reaction as was determined by TLC analysis, the reaction mixture was diluted with $\mathrm{CH}_{2} \mathrm{Cl}_{2}(3.0 \mathrm{~mL})$ and the organic layer was washed with aqueous saturated brine solution $(2 \times 3 \mathrm{~mL})$. The combined organic layer was next dried over anhydrous $\mathrm{Na}_{2} \mathrm{SO}_{4}$, filtered, and concentrated under reduced pressure. The crude product was then purification by silica gel flash column chromatography using EtOAc /Hexane as eluent (1:9) to afford the desired products.

Dimethyl

2-(3-methoxyphenyl)-2-(2-(1-methyl-1H-indol-3-yl)-2phenylvinyl)malonate (9a):

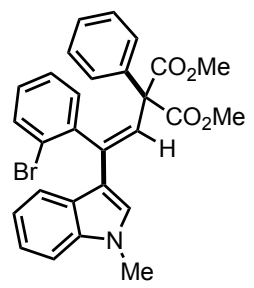

Following the general procedure, compound $4 \mathrm{p}(0.047 \mathrm{~g}, 0.1 \mathrm{mmol})$ was transformed into tri-substituted olefin $9 \mathrm{a}$ in $86 \%$ yield $(0.044 \mathrm{~g}$, gummy solid) as inseparable mixture of isomers (Z/E > 20:1). $R_{f} 0.35$ (EtOAc: Hexane 2:8); ${ }^{1} \mathrm{H}$ NMR $\left(\mathrm{CDCl}_{3}, 400\right.$ $\mathrm{MHz}) \delta 8.01(\mathrm{~d}, J=7.9 \mathrm{~Hz}, 1 \mathrm{H}), 7.62-7.44(\mathrm{~m}, 2 \mathrm{H}), 7.46(\mathrm{dd}, J=7.3,1.7 \mathrm{~Hz}, 1 \mathrm{H})$, $7.31(\mathrm{~d}, J=7.2 \mathrm{~Hz}, 2 \mathrm{H}), 7.27-7.20(\mathrm{~m}, 5 \mathrm{H}), 7.07-7.03(\mathrm{~m}, 2 \mathrm{H}), 6.93(\mathrm{dd}, J=7.1$, $2.1 \mathrm{~Hz}, 1 \mathrm{H}), 6.46(\mathrm{~s}, 1 \mathrm{H}), 3.71(\mathrm{~s}, 3 \mathrm{H}), 3.67(\mathrm{~s}, 3 \mathrm{H}), 3.47(\mathrm{~s}, 3 \mathrm{H}) ;{ }^{13} \mathrm{C}\left\{{ }^{1} \mathrm{H}\right\}$ NMR $\left(\mathrm{CDCl}_{3}, 100 \mathrm{MHz}\right) \delta 170.6,169.4,139.4,138.2,137.8,137.3,132.7,131.6,129.2$, 129.0, 128.1, 127.7, 126.6, 125.9, 124.7, 123.5, 122.2, 121.0, 120.4, 117.4, 109.7, 63.4, 53.3, 52.8, 33.0; HRMS (ESI-TOF) m/z: [M+H] ${ }^{+} \mathrm{C}_{28} \mathrm{H}_{25} \mathrm{BrNO}_{4}$ Calcd. 518.0967, Found 518.0967.

\section{Dimethyl} phenylmalonate (9b):

\section{2-(2-(2-bromophenyl)-2-(1-methyl-1H-indol-3-yl)vinyl)-2-}<smiles>COc1cccc(C(=Cc2cn(C)c3ccccc23)C(C)=O)c1</smiles>

Following the general procedure, compound $5 \mathrm{w}(0.047 \mathrm{~g}, 0.1 \mathrm{mmol})$ was transformed into tri-substituted olefin $9 \mathrm{~b}$ in $90 \%$ yield $(0.042 \mathrm{~g})$ as gummy solid. $R_{f}$ 0.30 (EtOAc: Hexane 2:8); FT-IR $\left(\mathrm{v} \mathrm{cm}^{-1}\right): 2951,1734,1582,1240,745 ;{ }^{1} \mathbf{H}$ NMR $\left(\mathrm{CDCl}_{3}, 400 \mathrm{MHz}\right) \delta 7.74(\mathrm{~d}, J=8.0 \mathrm{~Hz}, 1 \mathrm{H}), 7.24(\mathrm{~s}, 1 \mathrm{H}), 7.21-7.17(\mathrm{~m}, 4 \mathrm{H}), 7.15-$ 
$7.09(\mathrm{~m}, 6 \mathrm{H}), 6.99(\mathrm{~d}, J=7.3 \mathrm{~Hz}, 2 \mathrm{H}), 6.98(\mathrm{~s}, 1 \mathrm{H}) 6.77-6.74(\mathrm{~m}, 1 \mathrm{H}), 6.59(\mathrm{~s}, 1 \mathrm{H})$, $3.69(\mathrm{~s}, 3 \mathrm{H}), 3.64(\mathrm{~s}, 3 \mathrm{H}), 3.42(\mathrm{~s}, 6 \mathrm{H}) ;{ }^{13} \mathrm{C}\left\{{ }^{1} \mathrm{H}\right\}$ NMR $\left(\mathrm{CDCl}_{3}, 100 \mathrm{MHz}\right) \delta 170.3$, 159.2, 139.7, 139.3, 138.7, 137.7, 129.9, 129.8, 129.0, 127.6, 126.2, 122.2, 122.0, 121.6, 121.1, 120.3, 115.3, 113.2, 109.6, 63.8, 55.4, 52.9, 33.0; HRMS (ESI-TOF) $\mathrm{m} / \mathrm{z}:[\mathrm{M}+\mathrm{H}]^{+} \mathrm{C}_{29} \mathrm{H}_{28} \mathrm{NO}_{5}$ Calcd. 470.1967, Found 470.1962.

Dimethyl 2-(3-(4-(benzyloxy)phenyl)-3-(2,4,6-trimethoxyphenyl)allyl)-2-(3methoxyphenyl)malonate (9c):<smiles>COc1cccc(C(=O)CC(=Cc2c(O)cc([N+](=O)[O-])cc2[N+](=O)[O-])c2ccc([O-])cc2)c1</smiles>

Following the general procedure, compound $7 \mathrm{~h}(0.063 \mathrm{~g}, 0.1 \mathrm{mmol})$ was transformed into tri-substituted olefin $9 \mathrm{c}$ in $91 \%$ yield $\left(0.057 \mathrm{~g}\right.$ ) as gummy solid. $R_{f} 0.35$ (EtOAc: Hexane 2:8); ${ }^{1} \mathrm{H}$ NMR $\left(\mathrm{CDCl}_{3}, 400 \mathrm{MHz}\right) \delta 7.41-7.34(\mathrm{~m}, 4 \mathrm{H}), 7.30(\mathrm{~d}, J=7.1 \mathrm{~Hz}$, $1 \mathrm{H}), 7.23(\mathrm{t}, J=8.0 \mathrm{~Hz}, 1 \mathrm{H}), 7.07-7.01(\mathrm{~m}, 4 \mathrm{H}), 6.84-6.77(\mathrm{~m}, 3 \mathrm{H}), 6.18(\mathrm{~s}, 2 \mathrm{H})$, $5.97(\mathrm{t}, J=6.5 \mathrm{~Hz}, 1 \mathrm{H}), 5.00(\mathrm{~s}, 2 \mathrm{H}), 3.86(\mathrm{~s}, 3 \mathrm{H}), 3.78(\mathrm{~s}, 3 \mathrm{H}), 3.71(\mathrm{~s}, 6 \mathrm{H}), 3.67(\mathrm{~s}$, $6 \mathrm{H}), 2.99(\mathrm{~d}, J=6.5 \mathrm{~Hz}, 2 \mathrm{H}) ;{ }^{13} \mathrm{C}\left\{{ }^{1} \mathrm{H}\right\}$ NMR $\left(\mathrm{CDCl}_{3}, 100 \mathrm{MHz}\right) \delta 171.3,161.0,159.3$, 158.7, 157.8, 138.2, 137.4, 135.3, 135.1, 129.0, 128.6, 128.0., 127.6, 127.2, 123.7, 120.9, 115.1, 114.4, 112.9, 109.5, 90.9, 70.1, 62.4, 55.9, 55.4, 55.3, 52.8, 35.4; HRMS (ESI-TOF) m/z: $[\mathrm{M}+\mathrm{H}]^{+} \mathrm{C}_{37} \mathrm{H}_{39} \mathrm{O}_{9}$ Calcd. 627.2594, Found 627.2591. 


\section{HPLC data of the compounds}

Synthesis of enantiopure $(R)-\mathbf{4 a}$ : The enantiopure compound $(R)-\mathbf{4 b}$ was prepared from $(S)-1 \mathbf{b}$ following the general procedure as described for the preparation of the racemic compound $\mathbf{4 b}$.

$\mathrm{Ph}_{\mathrm{CO}_{2} \mathrm{Me}}^{\mathrm{CO}_{2} \mathrm{Me}}+\underset{\mathrm{Me}}{\stackrel{\mathrm{Ct}_{\mathrm{B}}}{\mathrm{Et}_{2} \mathrm{O}}}$

(S)-1b $(97 \%$ ee)

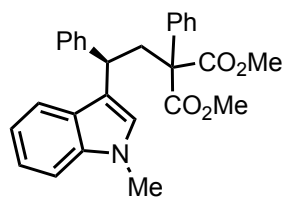

$(R)-4 \mathbf{b}(81 \%, 94 \%$ ee $)$

Scheme S1. Stereoselective 1,3-bisarylation reaction

HPLC data for $(R)-\mathbf{4 b}$ : Enantiomeric excess (94\%) was determined by HPLC analysis using a Chiralcel $\mathrm{OJ}-\mathrm{H}-3$ column, with IPA/Hex $=5: 95$ at a flow rate 1.0 $\mathrm{mL} / \mathrm{min}$ detected at $254 \mathrm{~nm}$ wavelength. Elution time: $9.68 \mathrm{~min}$.

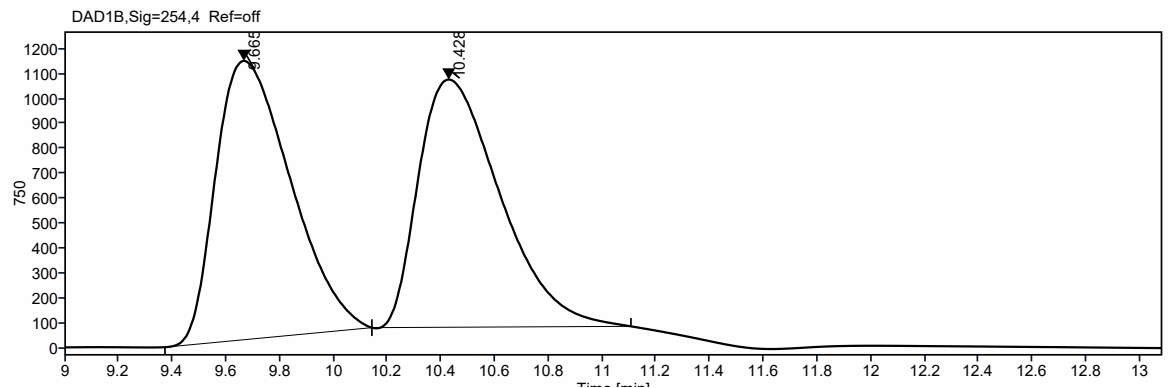

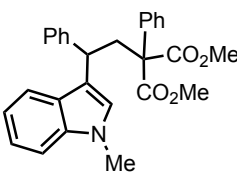

4b

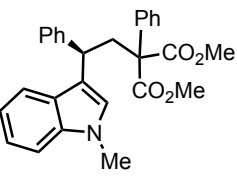

$(R)-\mathbf{4 b}$

Signal: DAD1B,Sig=254,4 Ref=off

RT [min] Type Width [min]

$\begin{array}{lll}9.684 & \mathrm{MM} \mathrm{m} & 0.31\end{array}$

10.687 MM M $\quad 0.54$ 
12. NMR spectra of new compounds

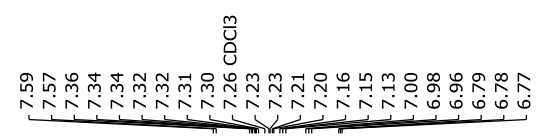

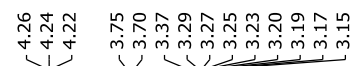

$\underbrace{\underbrace{n}}$

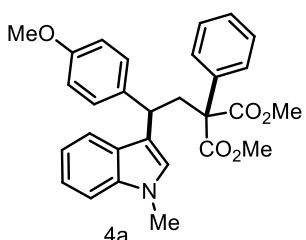

(400 $\mathrm{MHz}, \mathrm{CDCl}_{3}$ )
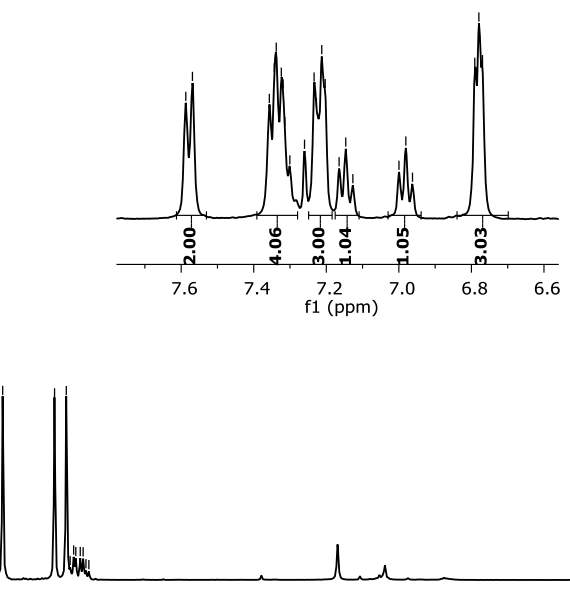

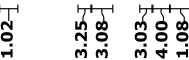

13.

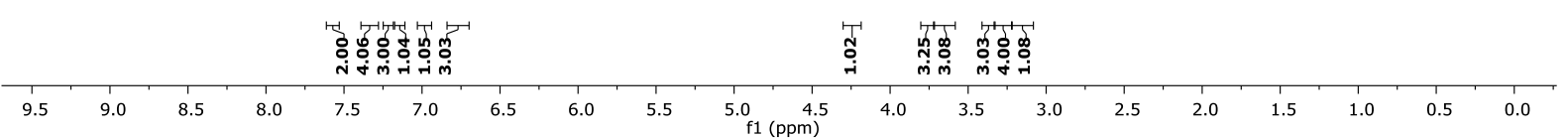

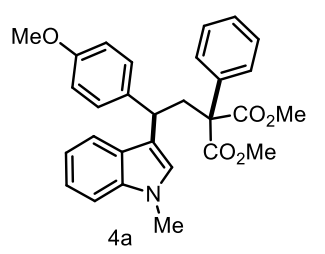

(100 $\mathrm{MHz}, \mathrm{CDCl}_{3}$ )

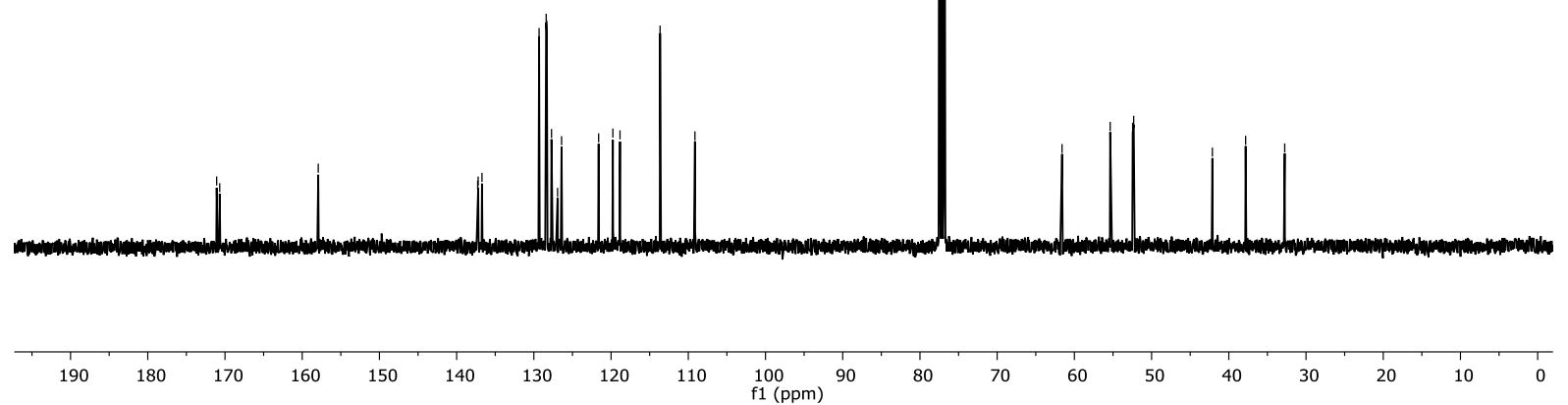




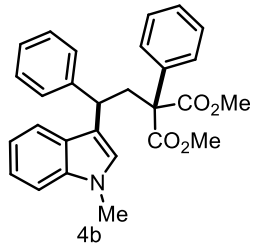

(400 MHz, $\mathrm{CDCl}_{3}$ )

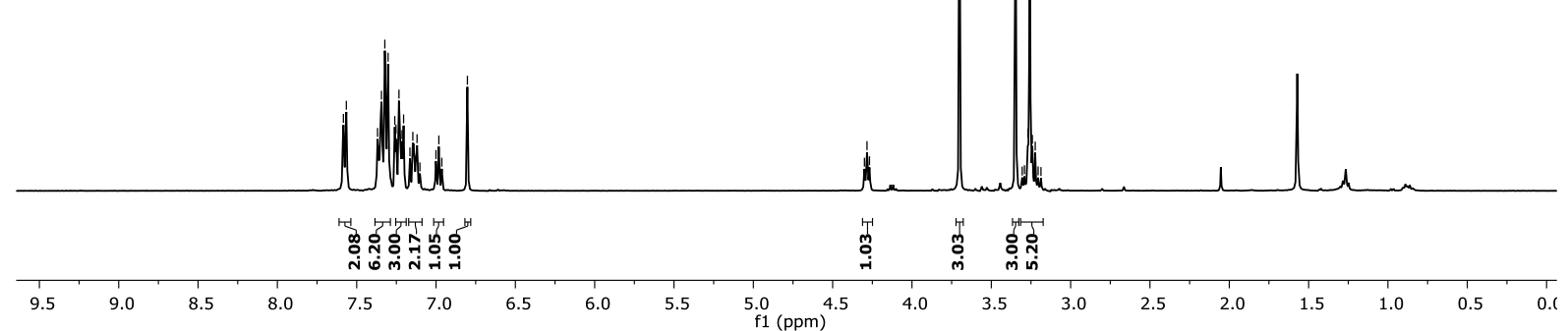

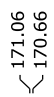

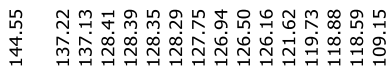

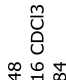

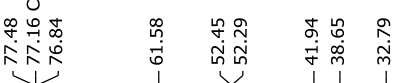

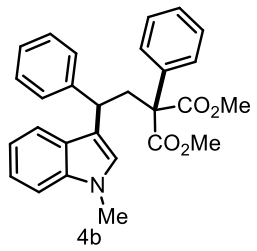

(100 MHz, $\mathrm{CDCl}_{3}$ )

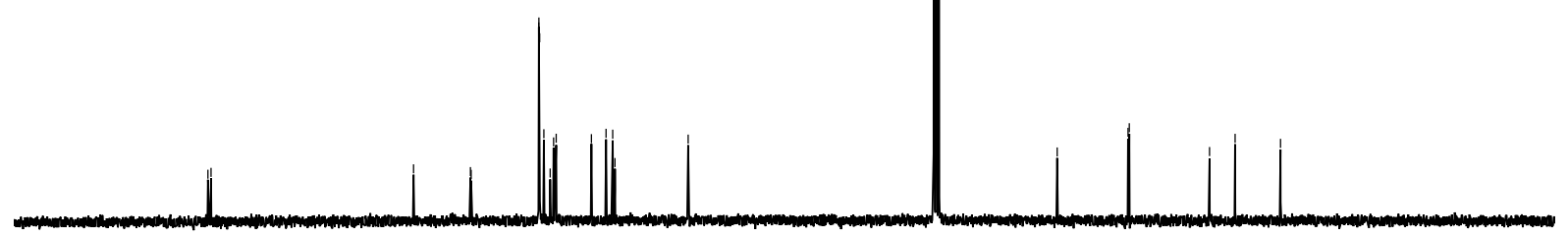

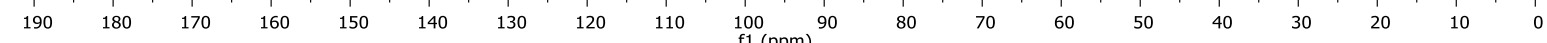




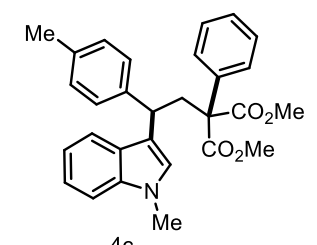

(400 $\mathrm{MHz}, \mathrm{CDCl}_{3}$ )
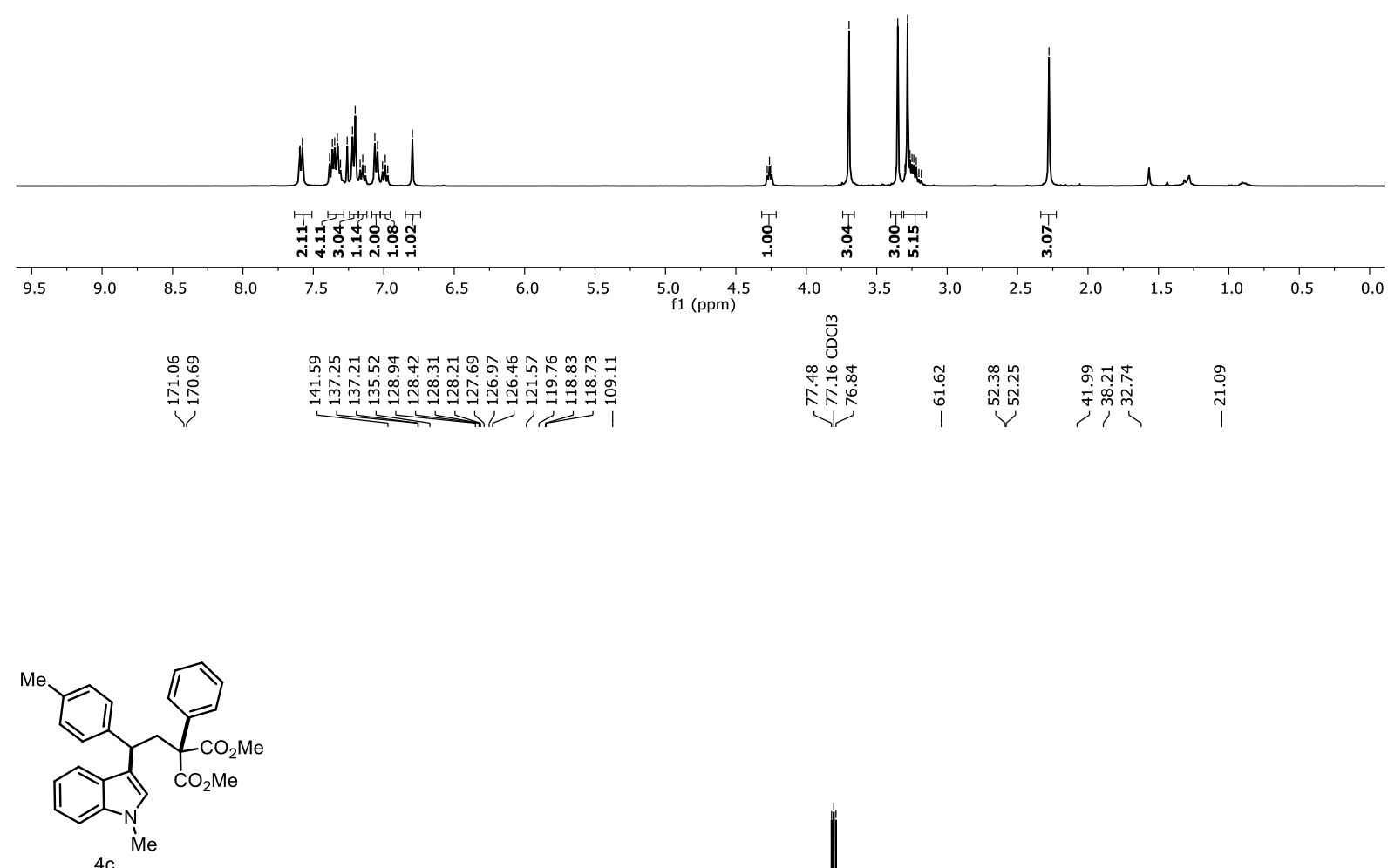

$\left(100 \mathrm{MHz}, \mathrm{CDCl}_{3}\right)$

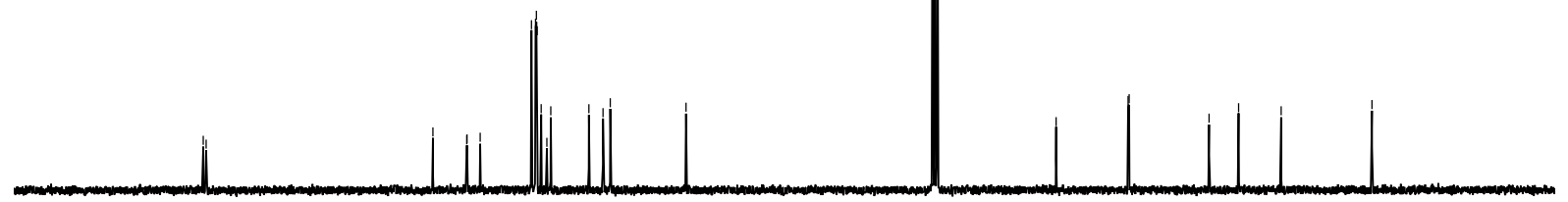

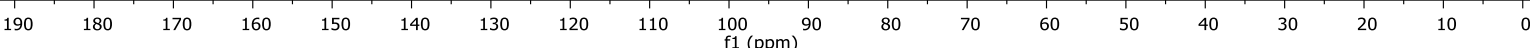



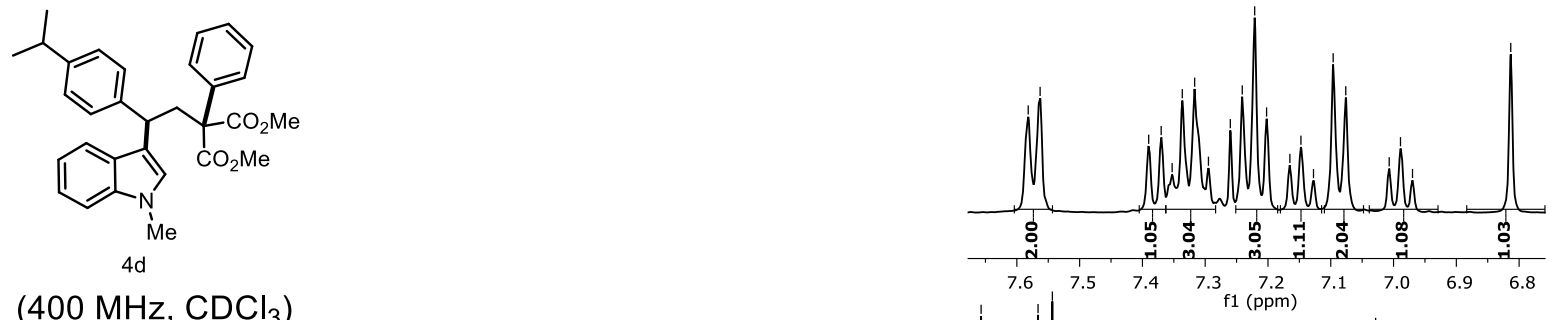

(400 $\mathrm{MHz}, \mathrm{CDCl}_{3}$ )
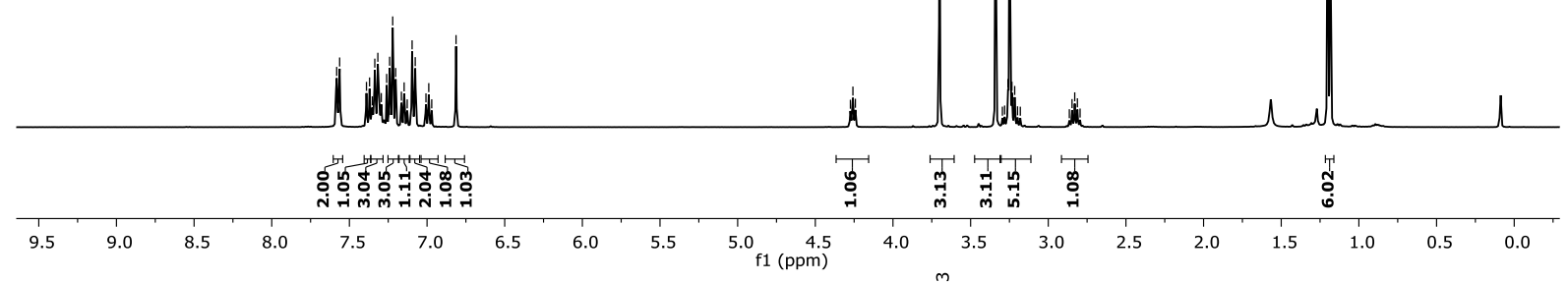

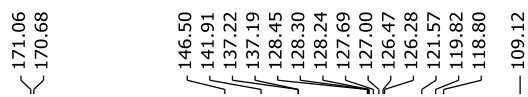

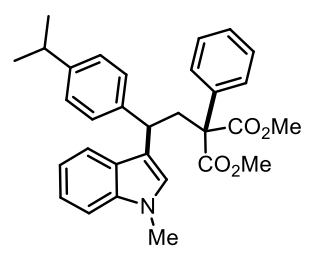

$4 d$

(100 MHz, $\mathrm{CDCl}_{3}$ )

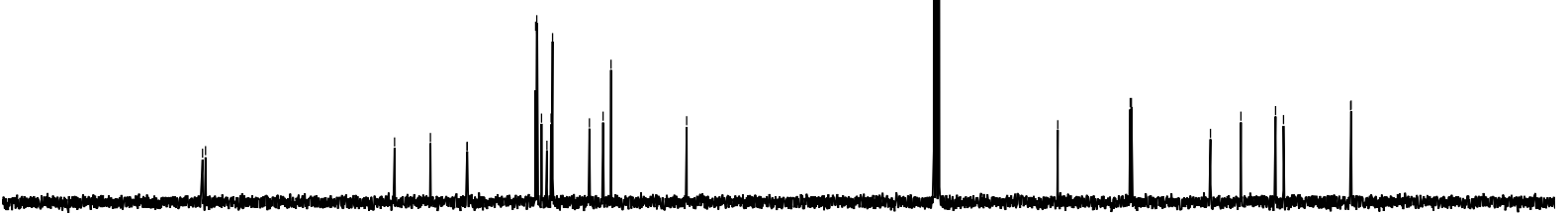

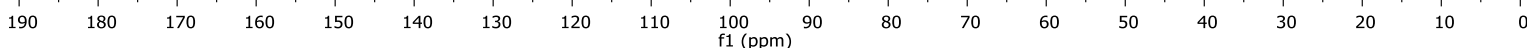




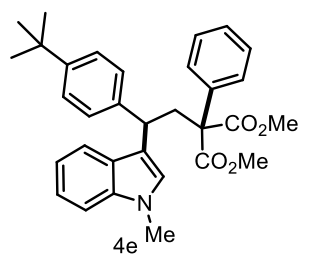

(400 $\mathrm{MHz}, \mathrm{CDCl}_{3}$ )
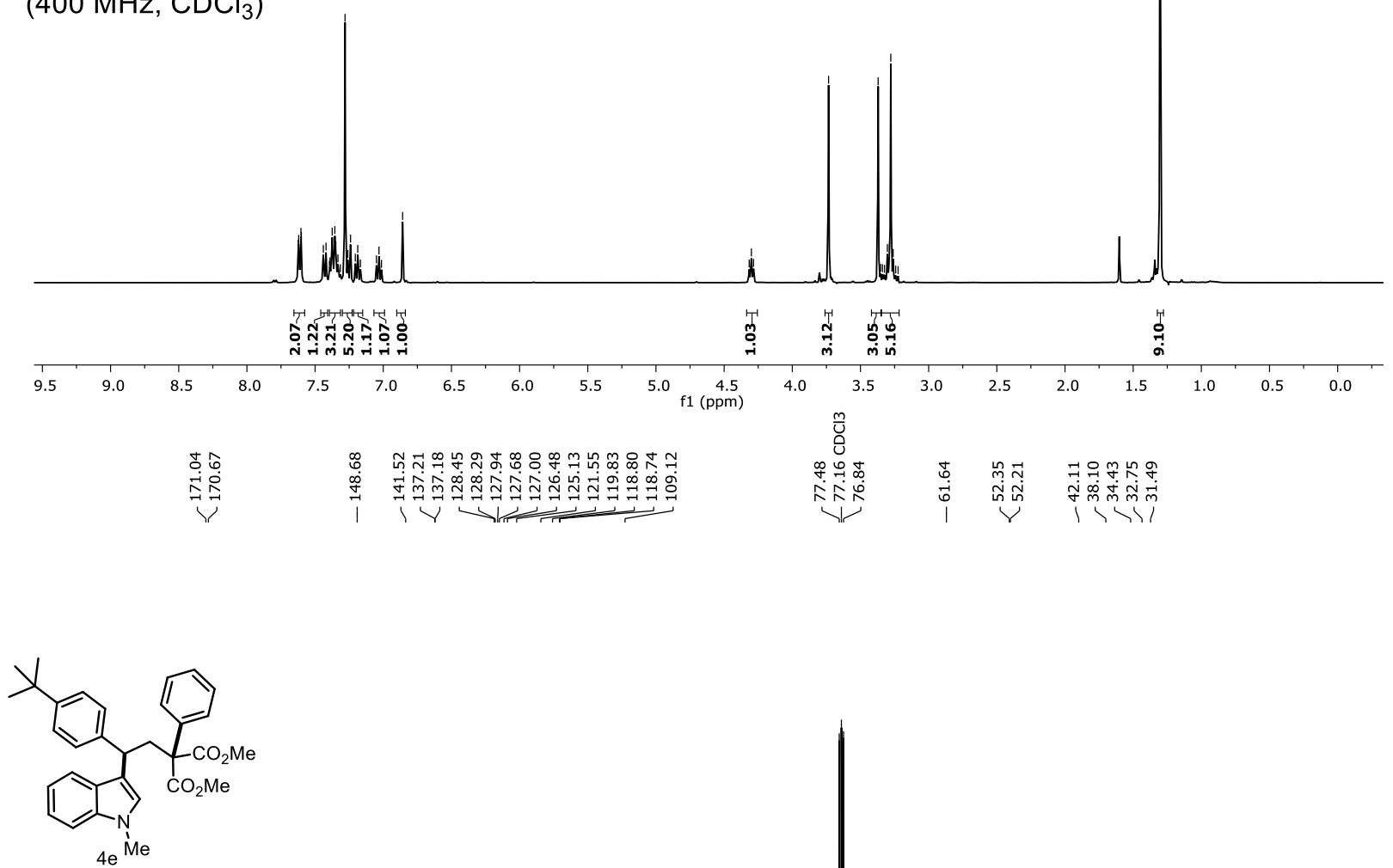

$\left(100 \mathrm{MHz}, \mathrm{CDCl}_{3}\right)$

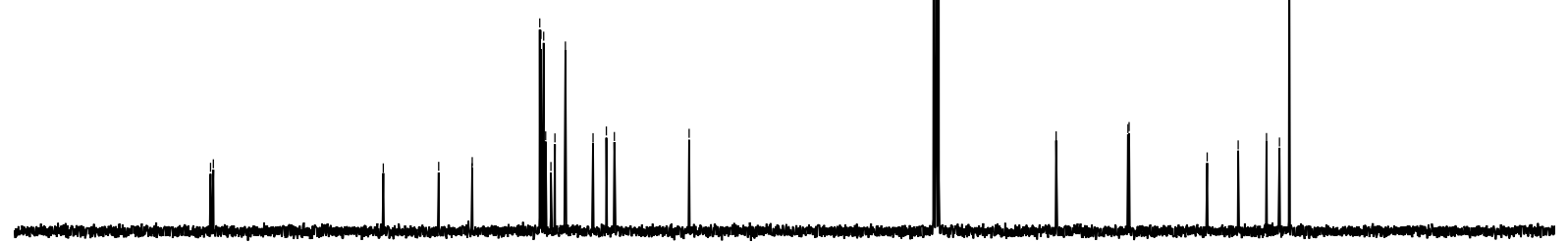

$\begin{array}{lllllllllllllllllllll}190 & 180 & 170 & 160 & 150 & 140 & 130 & 120 & 110 & 100 & 90 & 80 & 70 & 60 & 50 & 40 & 30 & 20 & 10 & 0\end{array}$ 

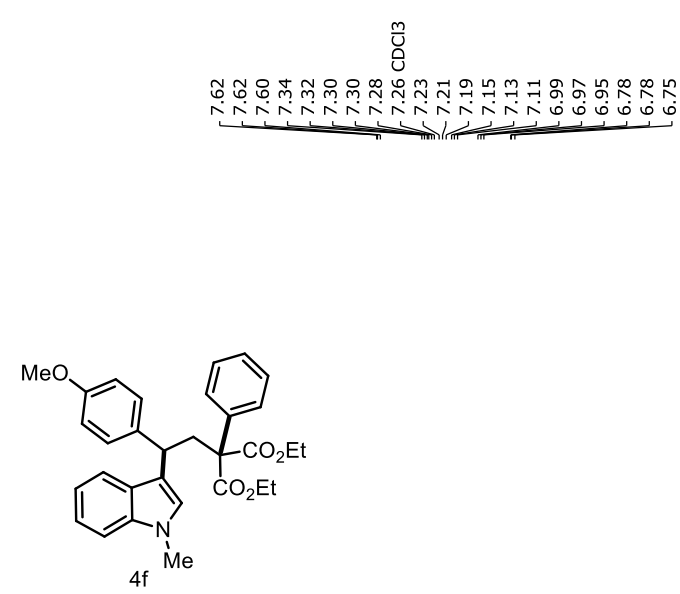

$\left(400 \mathrm{MHz}, \mathrm{CDCl}_{3}\right)$

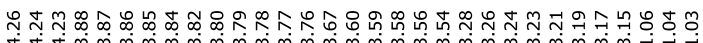

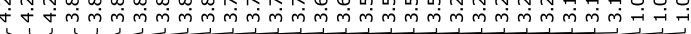

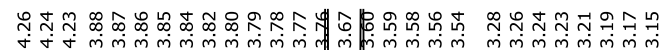

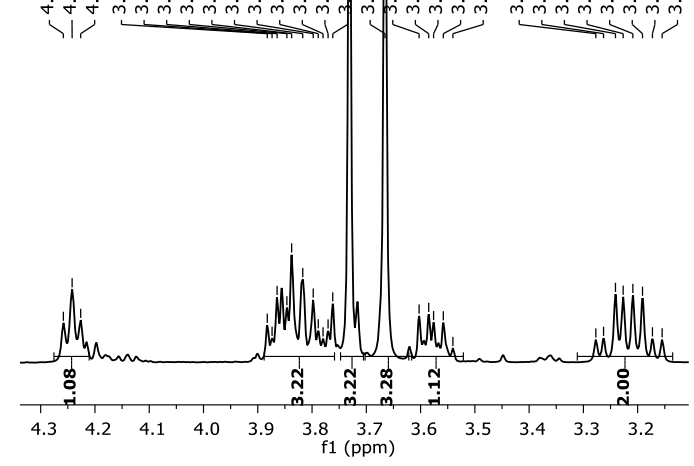

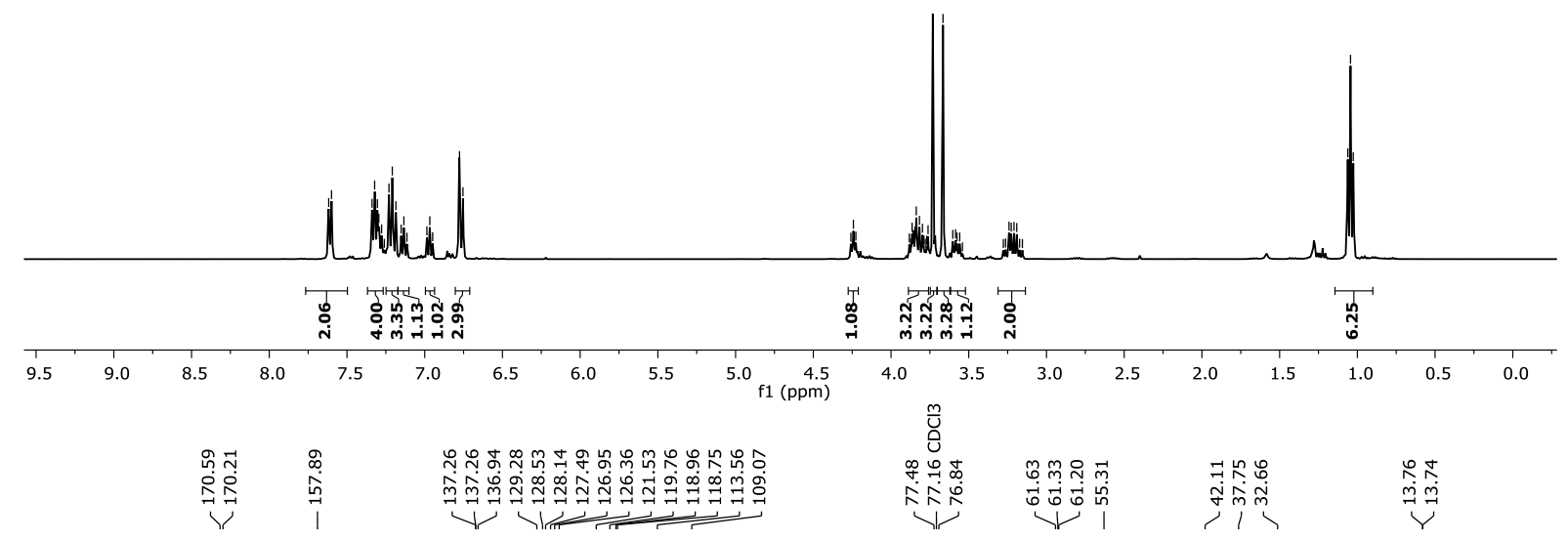

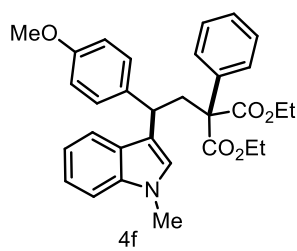

(100 MHz, $\left.\mathrm{CDCl}_{3}\right)$

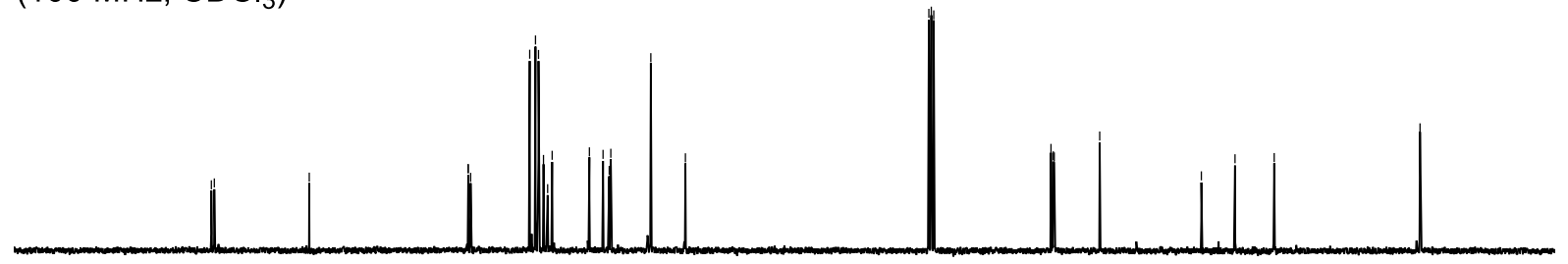

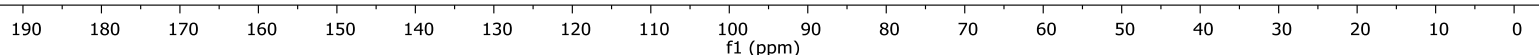




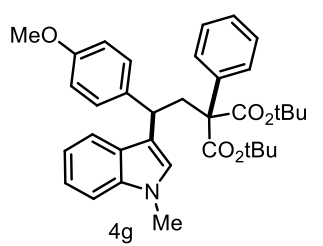

(400 MHz, $\mathrm{CDCl}_{3}$ )
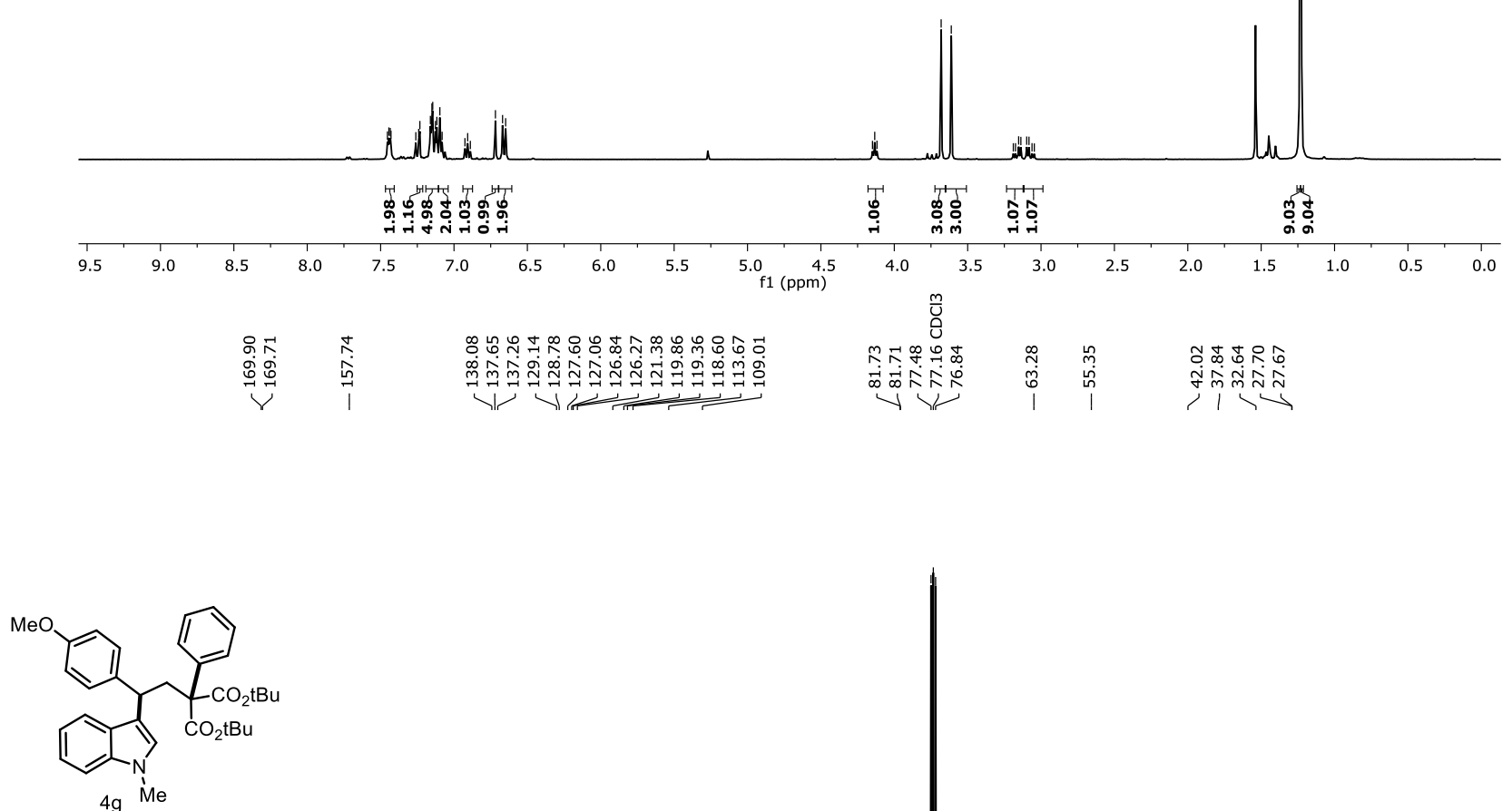

$\left(100 \mathrm{MHz}, \mathrm{CDCl}_{3}\right)$

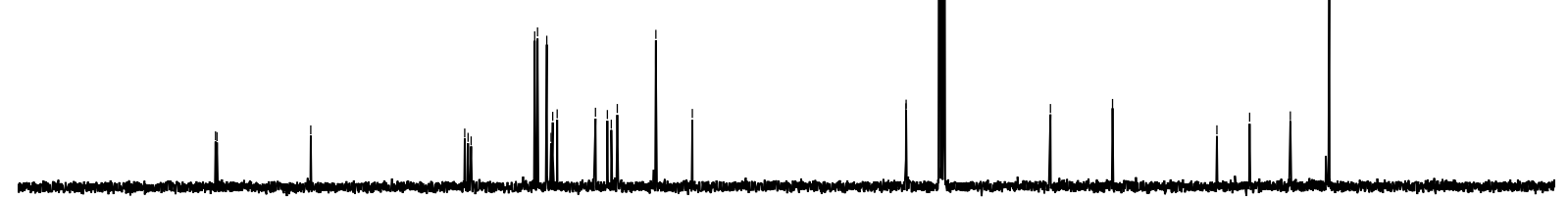

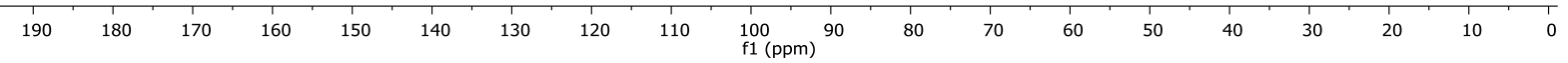



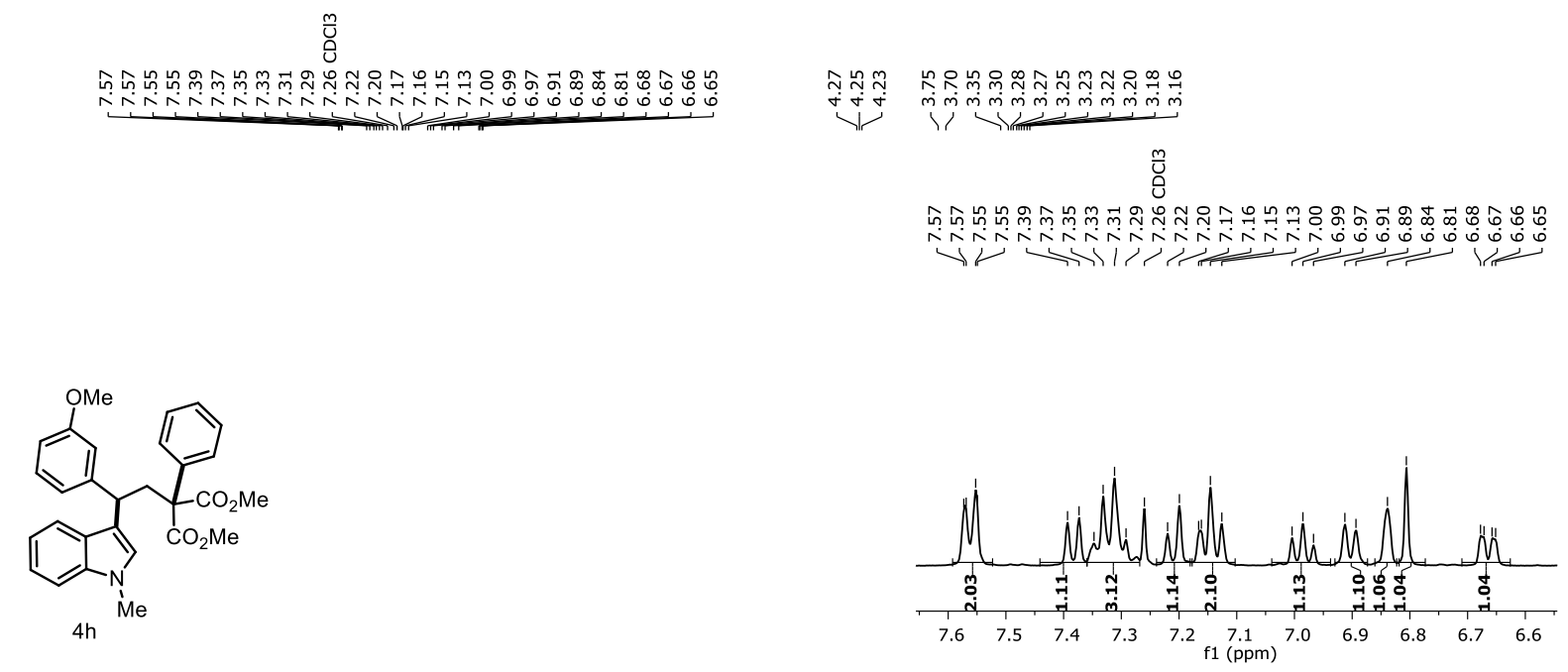

(400 MHz, $\mathrm{CDCl}_{3}$ )

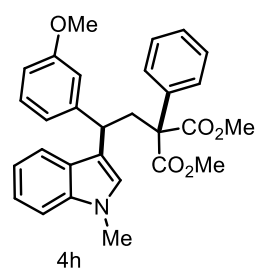

(100 MHz, $\mathrm{CDCl}_{3}$ )

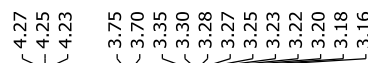

范

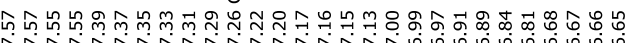

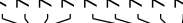

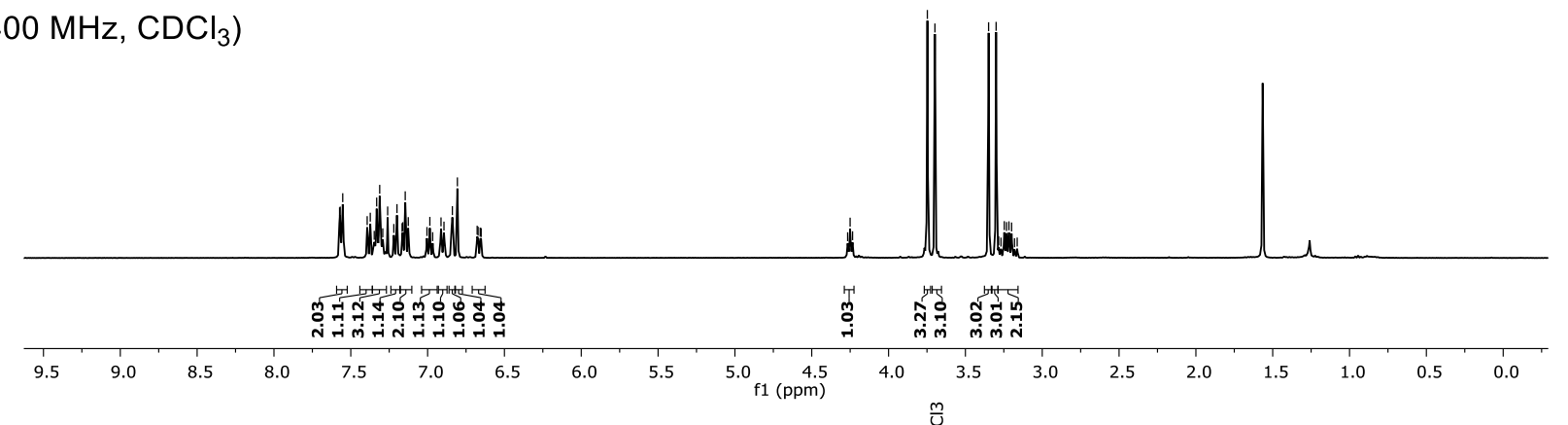

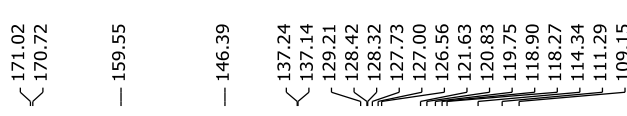

然

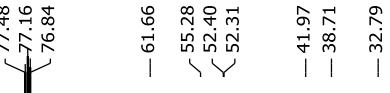

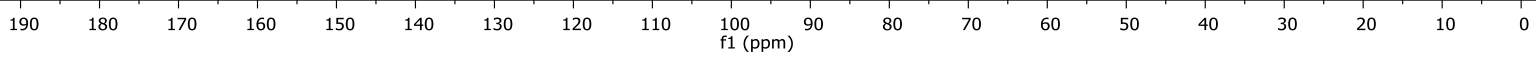




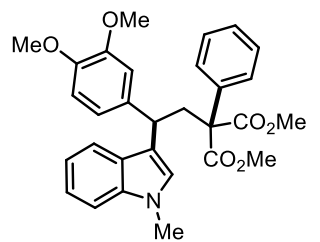

(400 MHz, $\left.\mathrm{CDCl}_{3}\right)$

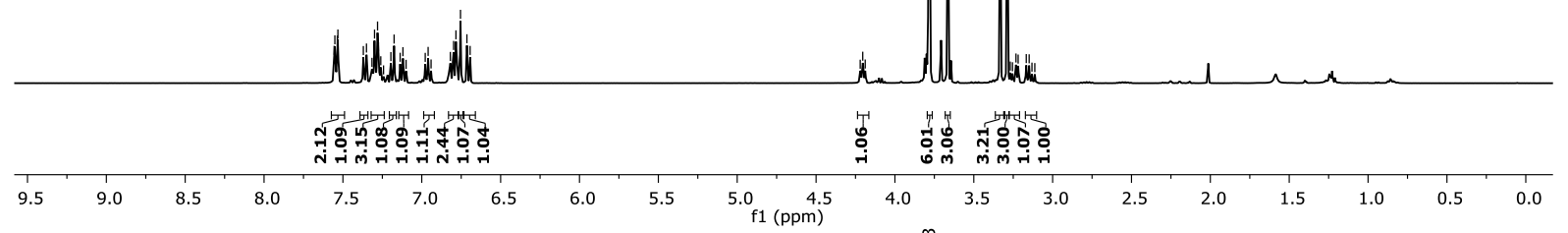

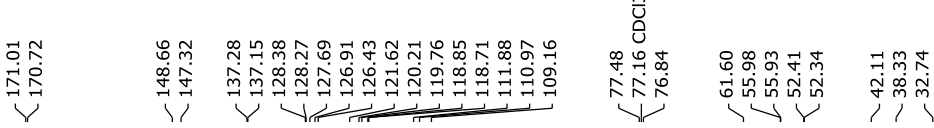

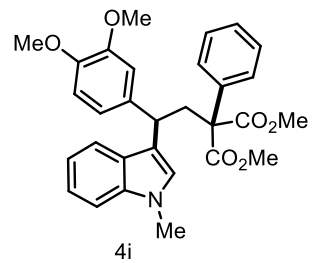

(100 MHz, $\mathrm{CDCl}_{3}$ )

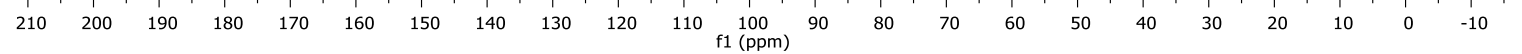




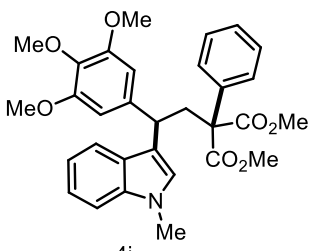

$4 \mathrm{j}$

(400 MHz, $\mathrm{CDCl}_{3}$ )
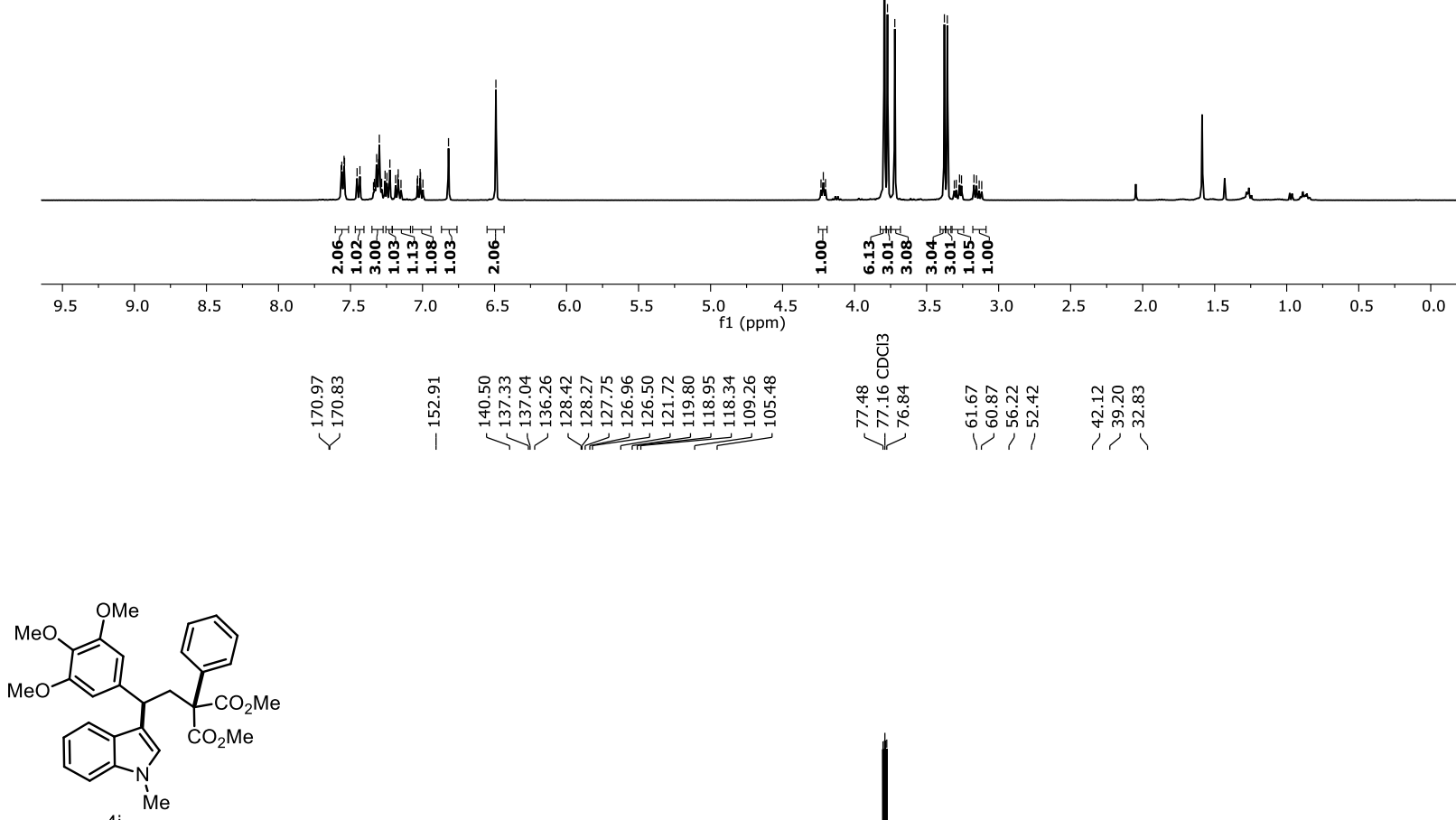

$\left(100 \mathrm{MHz}, \mathrm{CDCl}_{3}\right)$

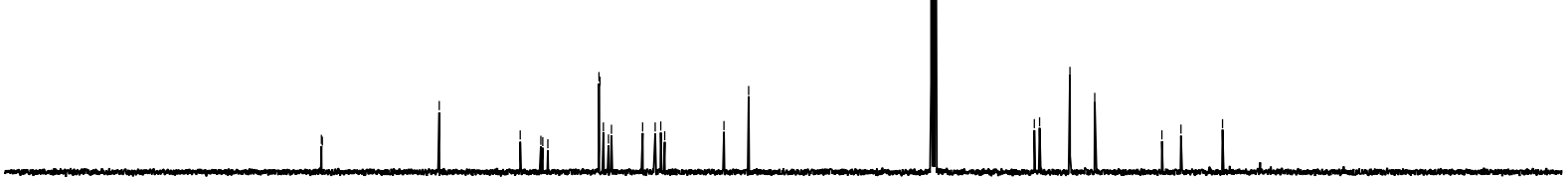

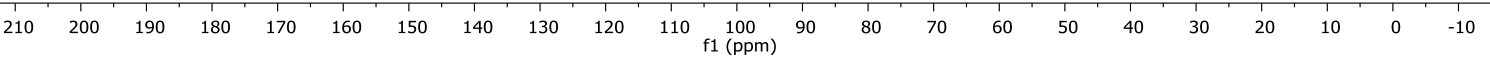




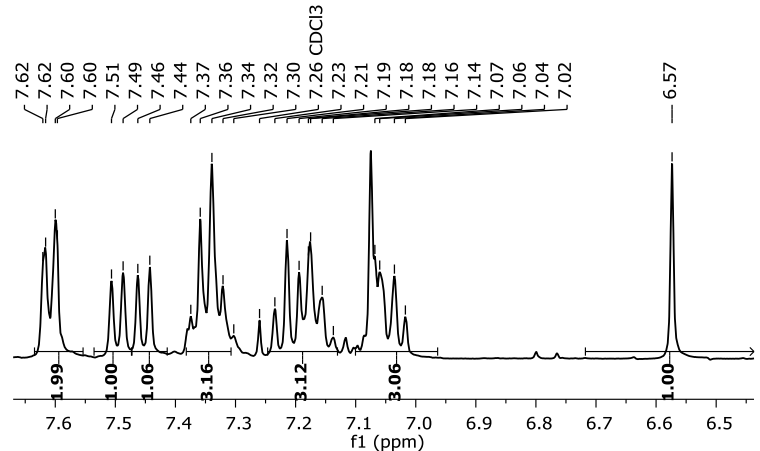

(400 MHz, $\mathrm{CDCl}_{3}$ )
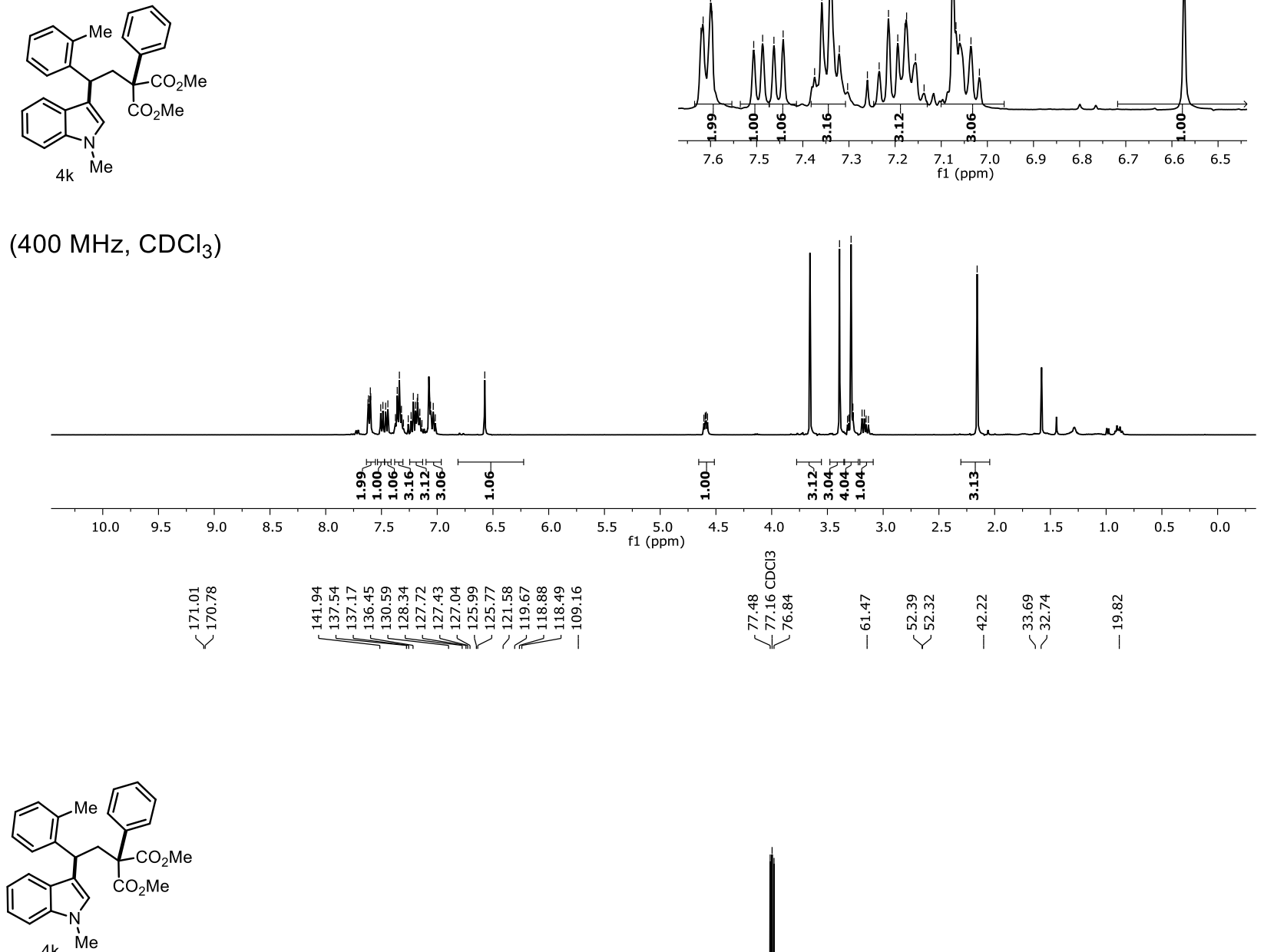

$\left(100 \mathrm{MHz}, \mathrm{CDCl}_{3}\right)$

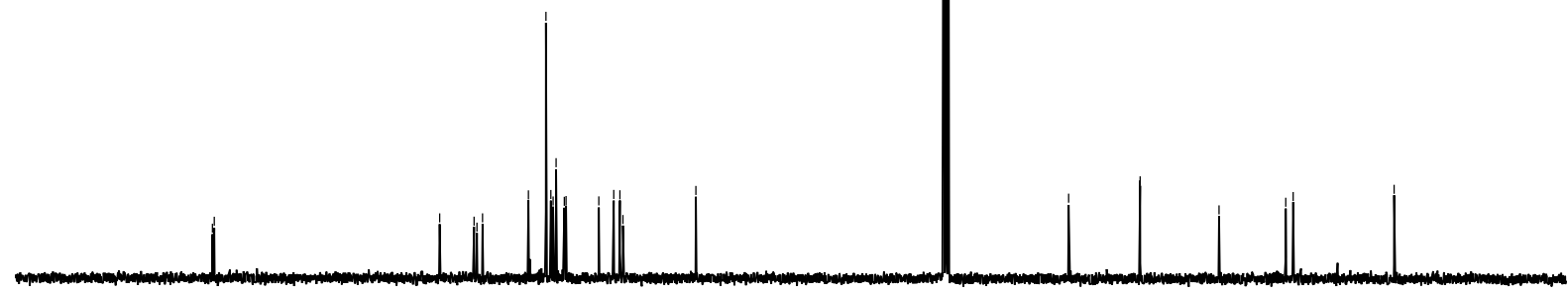

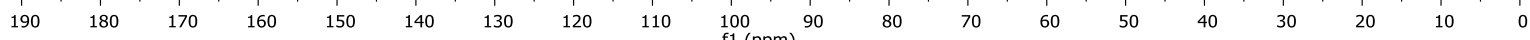




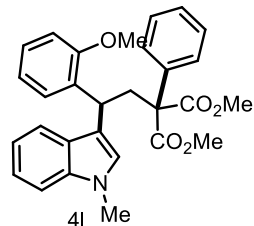

(400 MHz, $\mathrm{CDCl}_{3}$ )

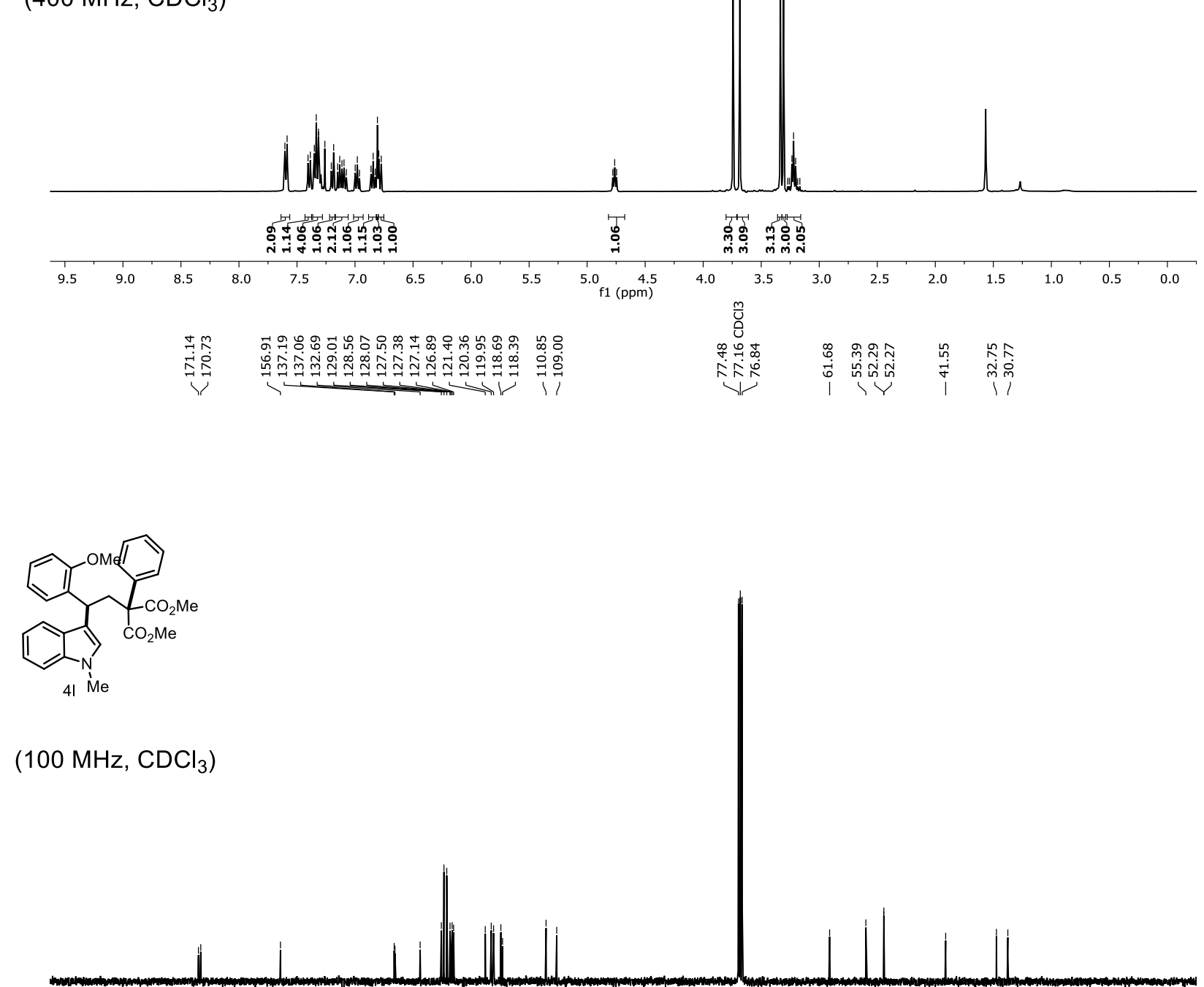

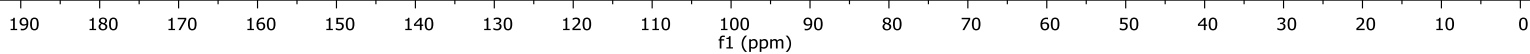




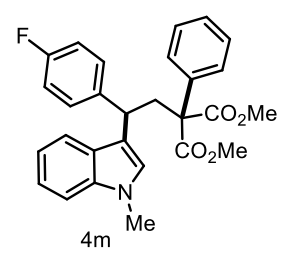

(400 MHz, $\mathrm{CDCl}_{3}$ )
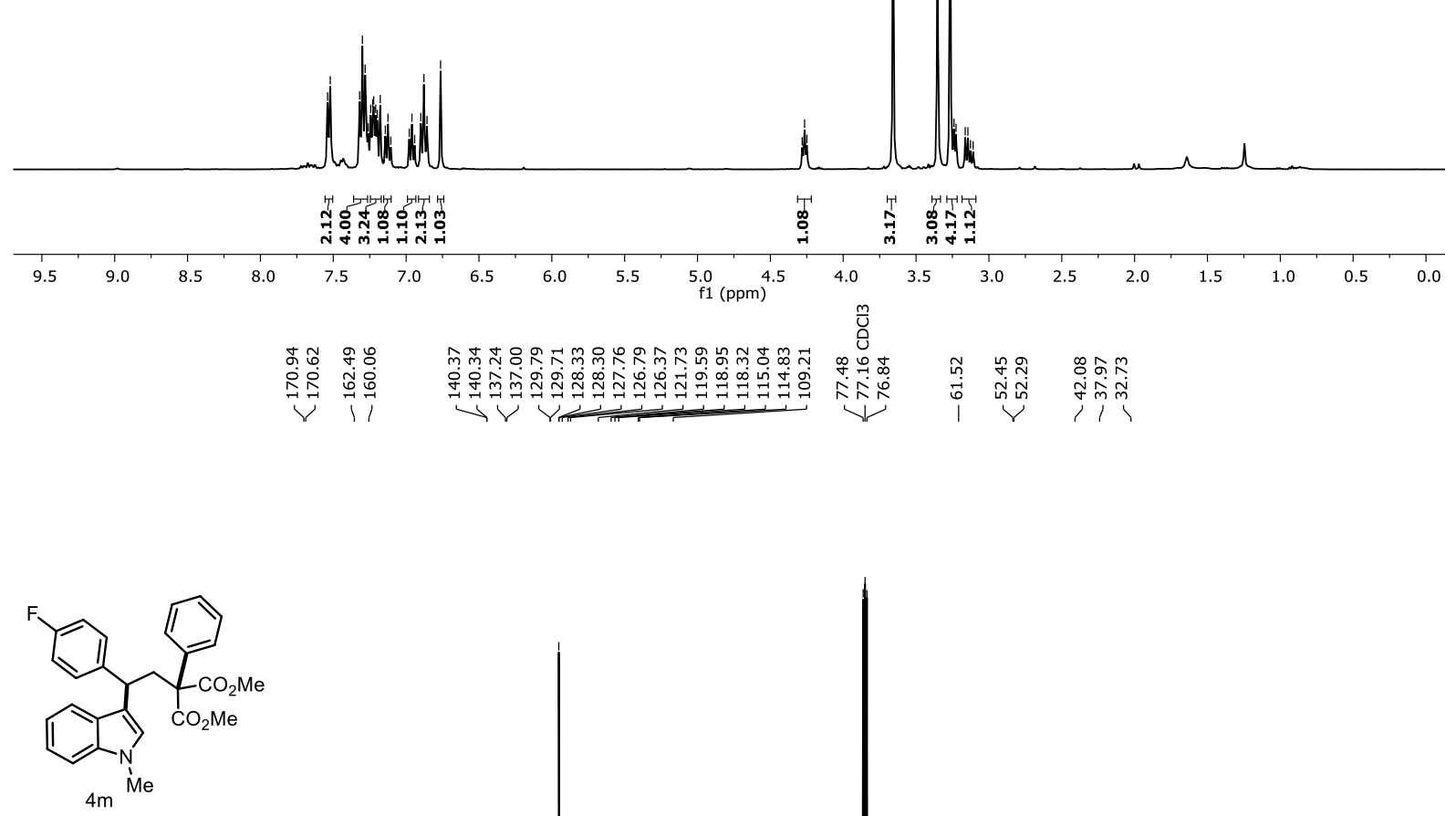

(100 MHz, $\mathrm{CDCl}_{3}$ )

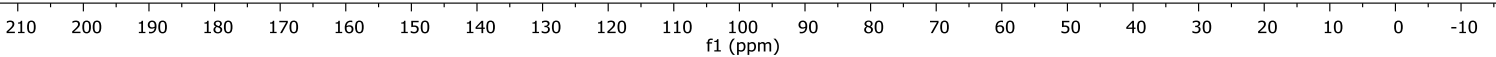


摘

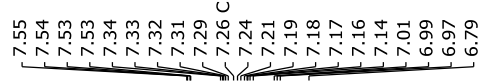

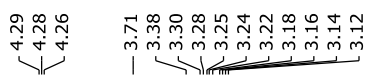

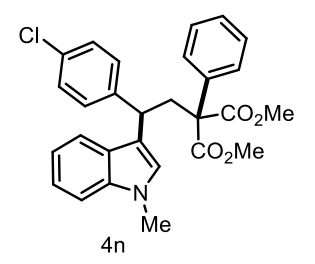

(400 MHz, $\mathrm{CDCl}_{3}$ )
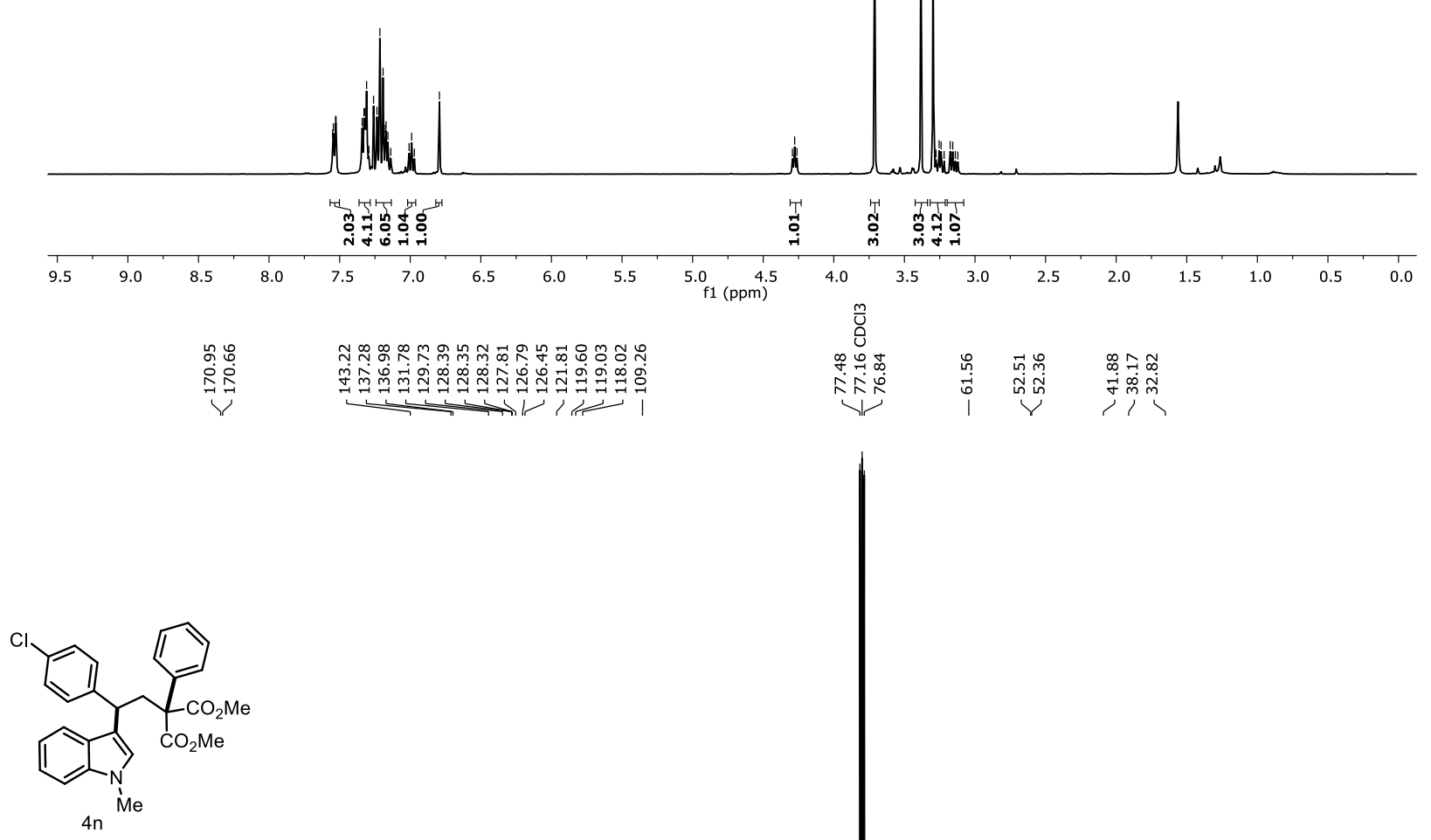

(100 MHz, $\mathrm{CDCl}_{3}$ )
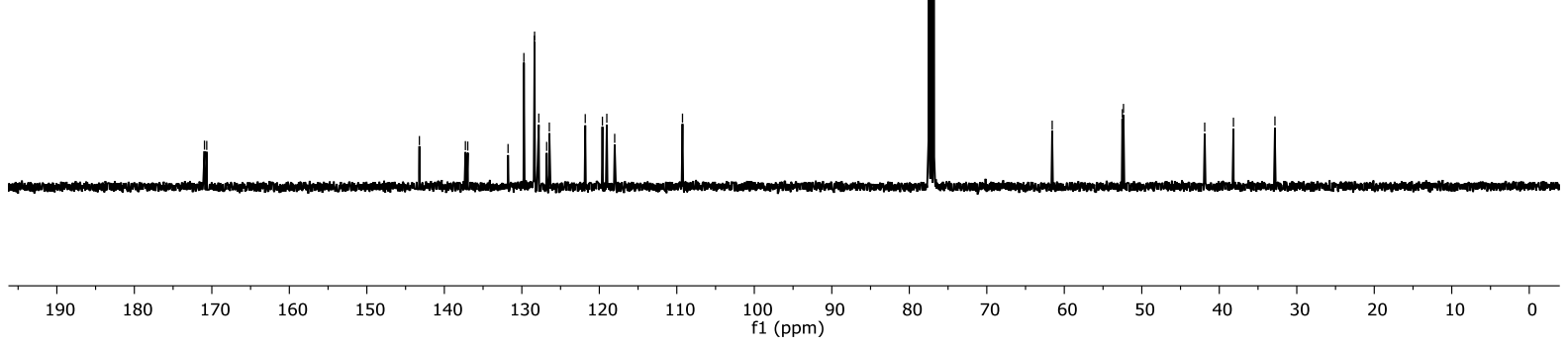

51 


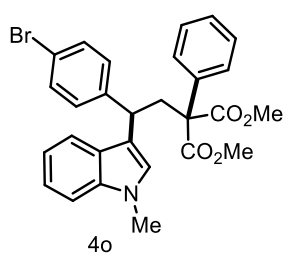

(400 MHz, $\mathrm{CDCl}_{3}$ )

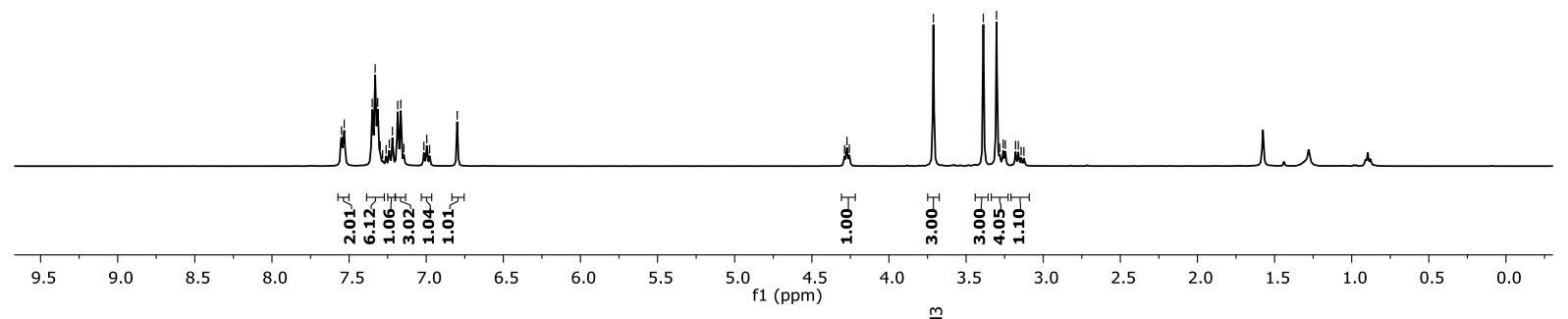

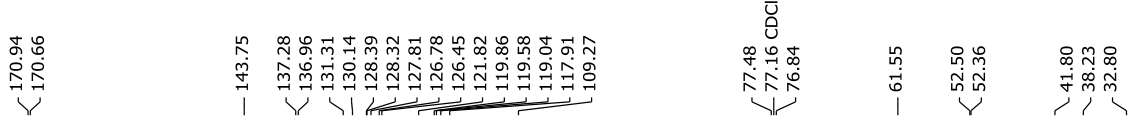

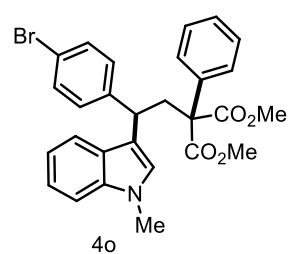

(100 MHz, $\mathrm{CDCl}_{3}$ )

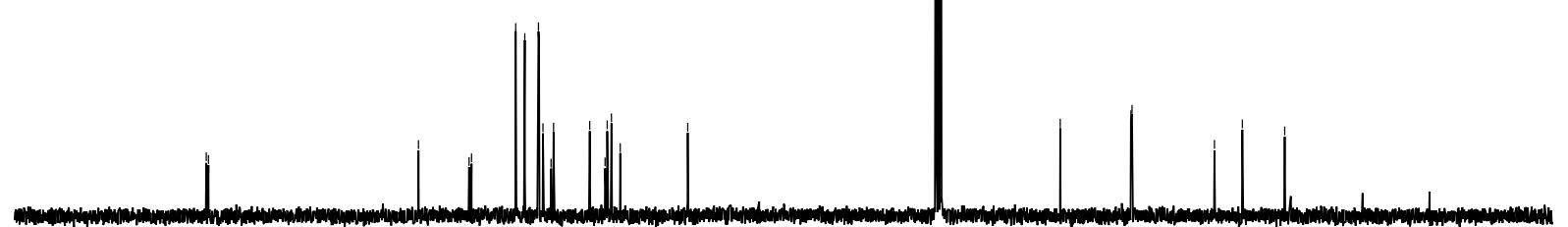

$\begin{array}{llllllllllllllllllll}190 & 180 & 170 & 160 & 150 & 140 & 130 & 120 & 110 & 100 & 90 & 80 & 70 & 60 & 50 & 40 & 30 & 20 & 10 & 0\end{array}$ 


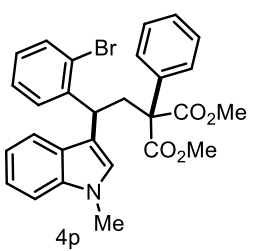

$$
\left(400 \mathrm{MHz}, \mathrm{CDCl}_{3}\right)
$$

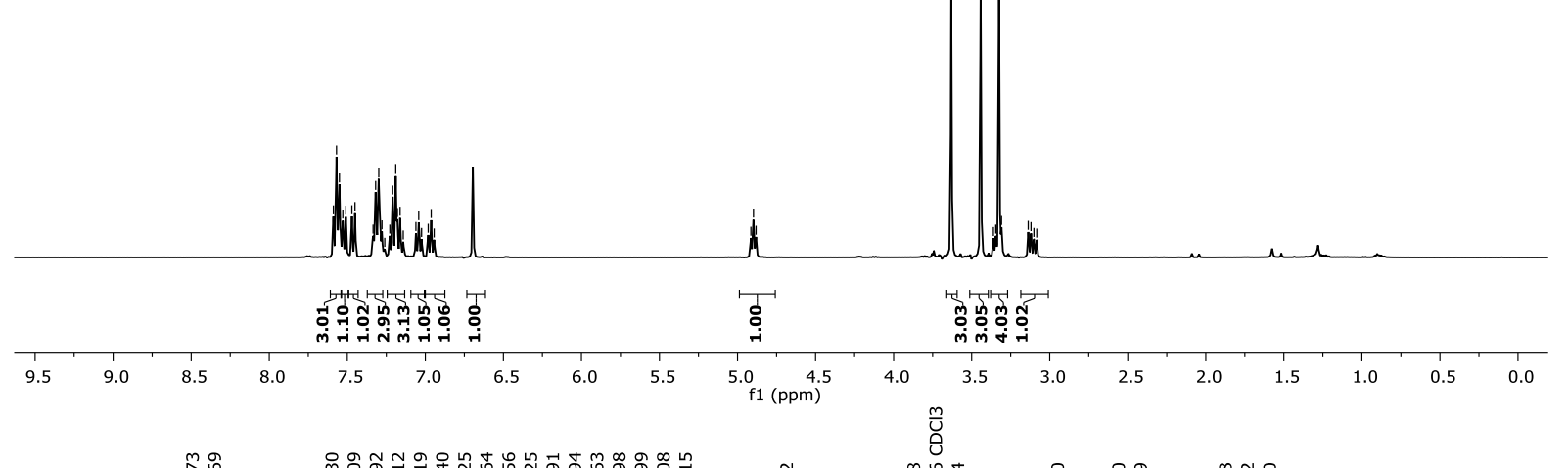

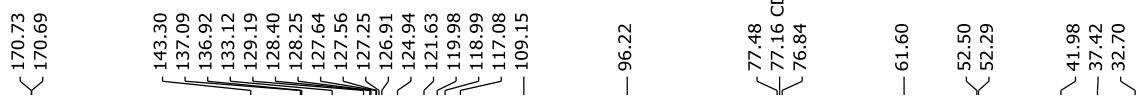

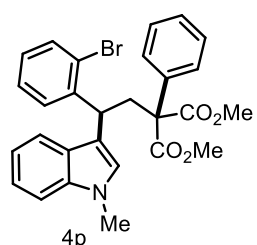

$\left(100 \mathrm{MHz}, \mathrm{CDCl}_{3}\right)$

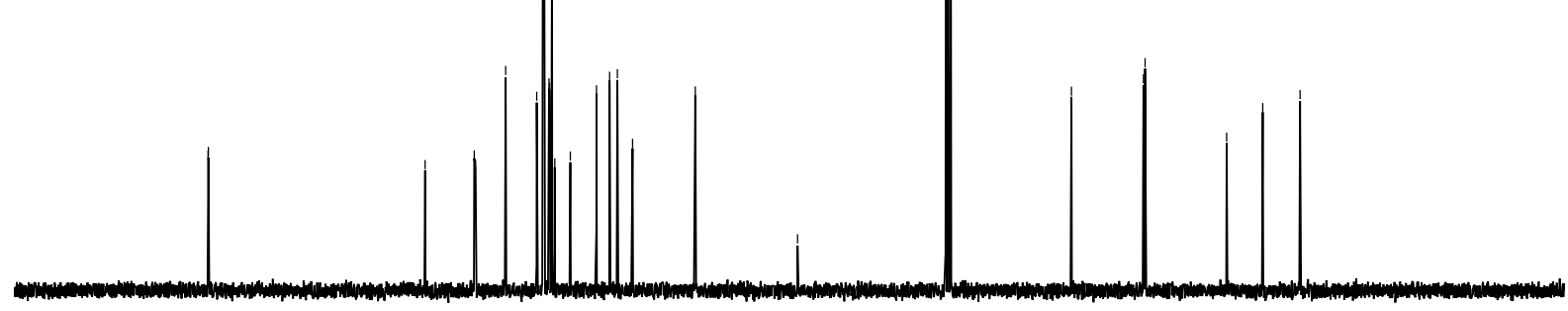

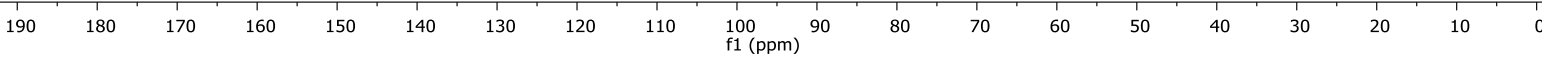




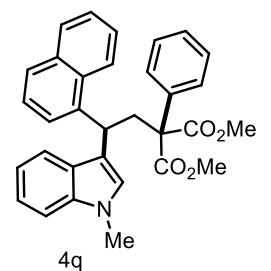

$4 q$

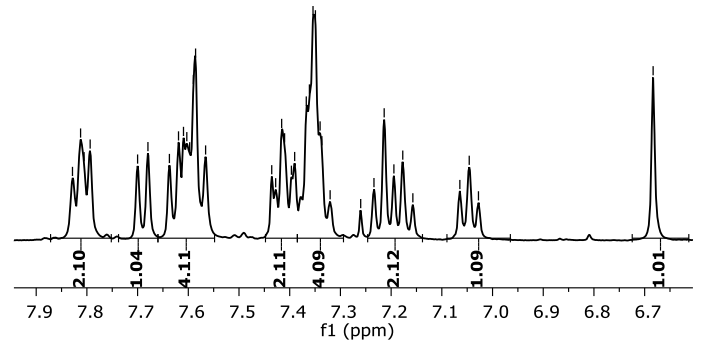

(400 MHz, $\mathrm{CDCl}_{3}$ )

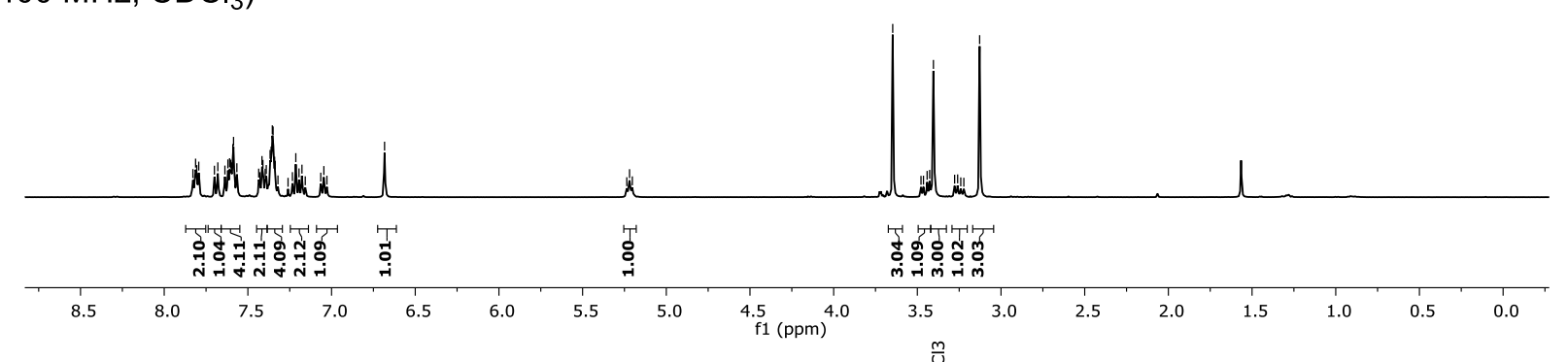

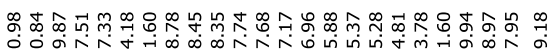

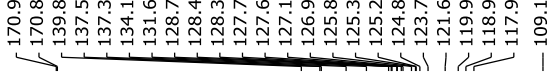

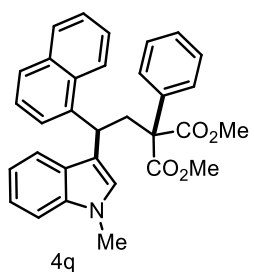

$4 q^{\mathrm{Me}}$

$\left(100 \mathrm{MHz}, \mathrm{CDCl}_{3}\right)$

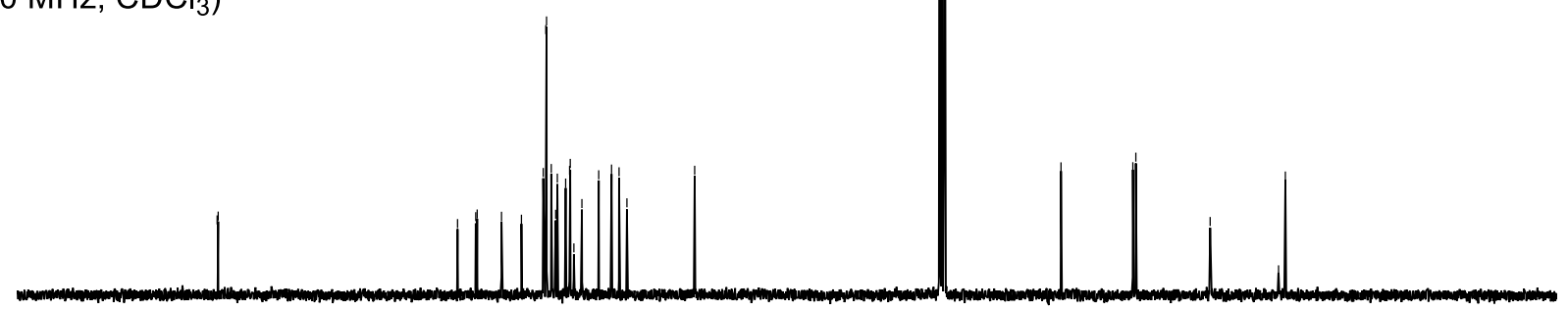




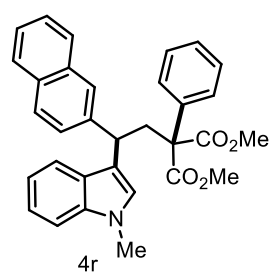

(400 MHz, $\mathrm{CDCl}_{3}$ )
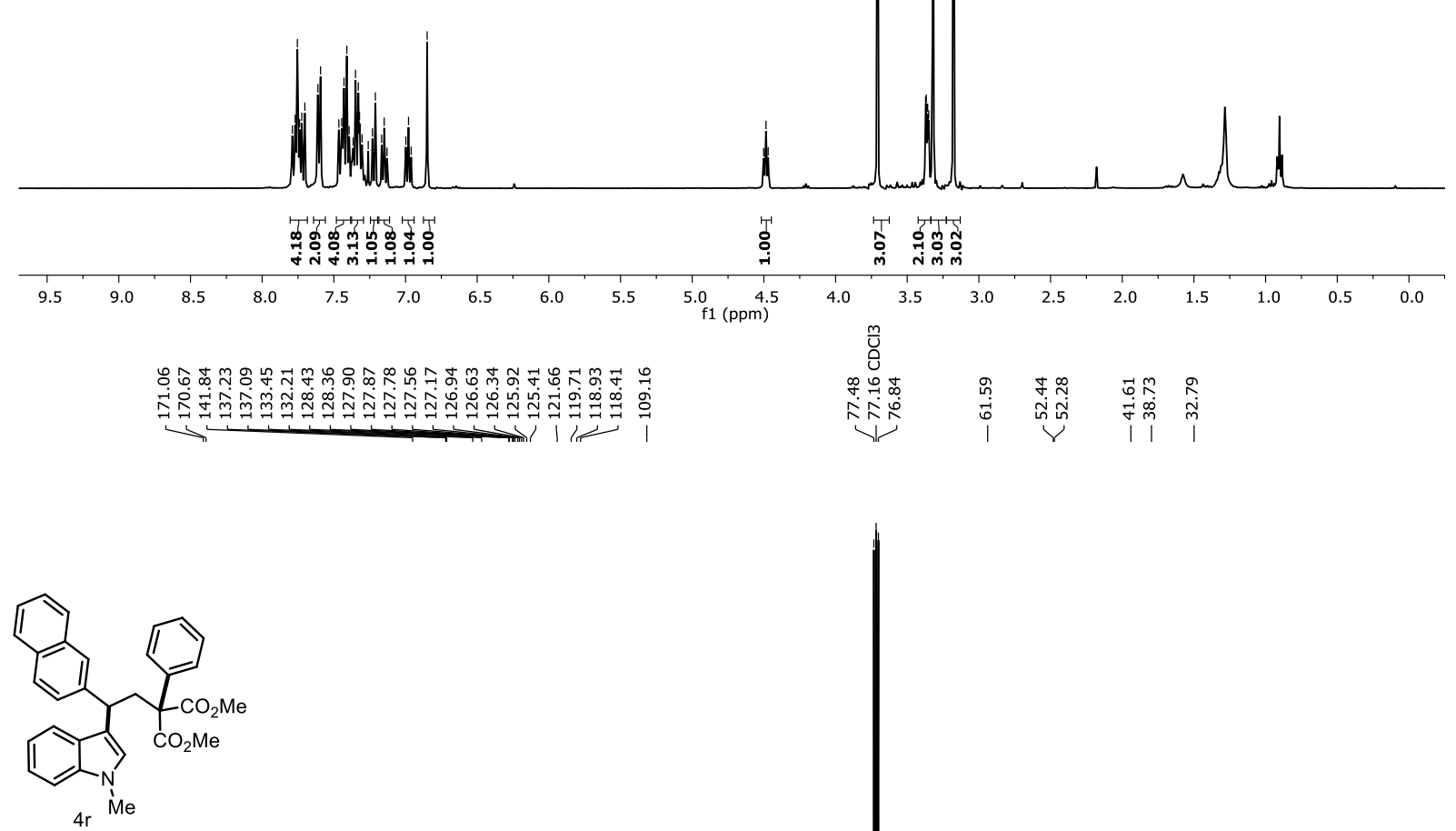

(100 MHz, $\mathrm{CDCl}_{3}$ )

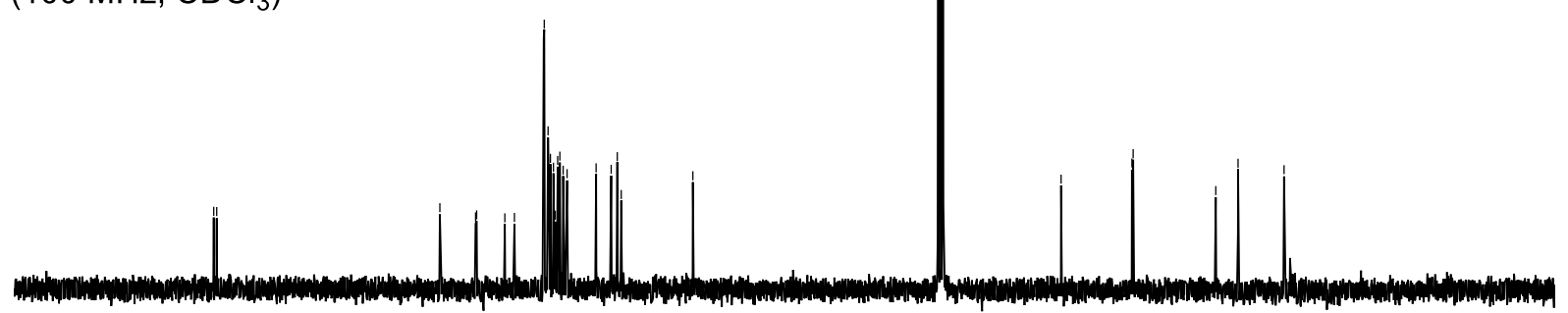

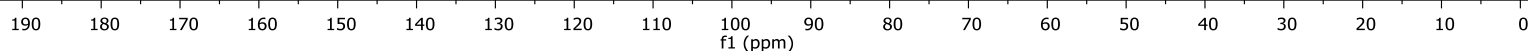




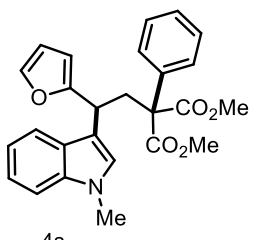

$4 \mathrm{~s}$

(400 MHz, $\mathrm{CDCl}_{3}$ )

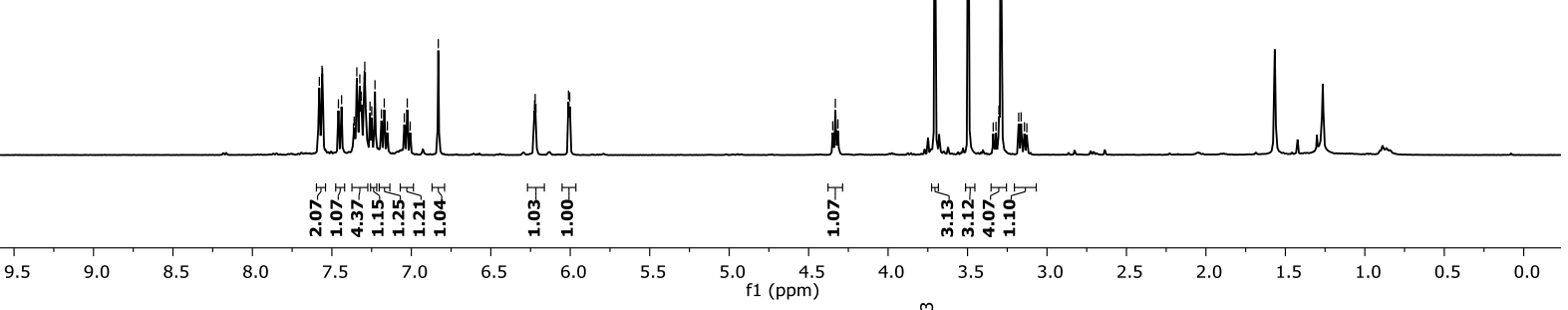

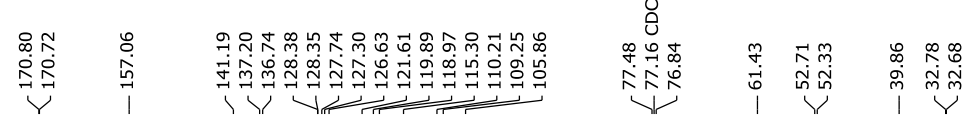

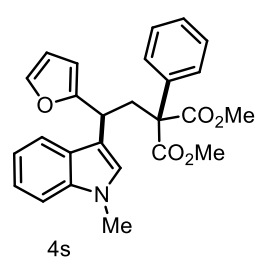

$\left(100 \mathrm{MHz}, \mathrm{CDCl}_{3}\right)$

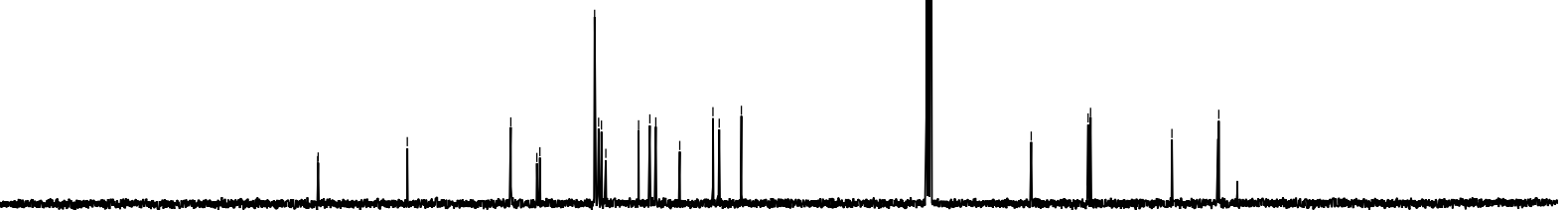

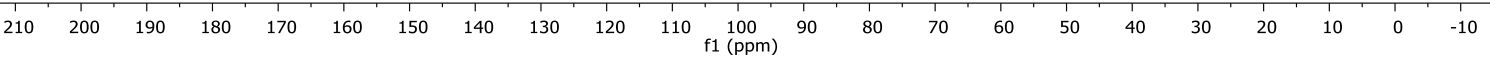




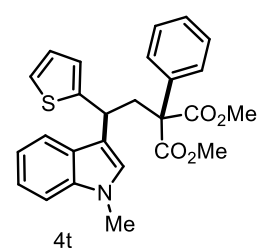

(400 MHz, $\mathrm{CDCl}_{3}$ )
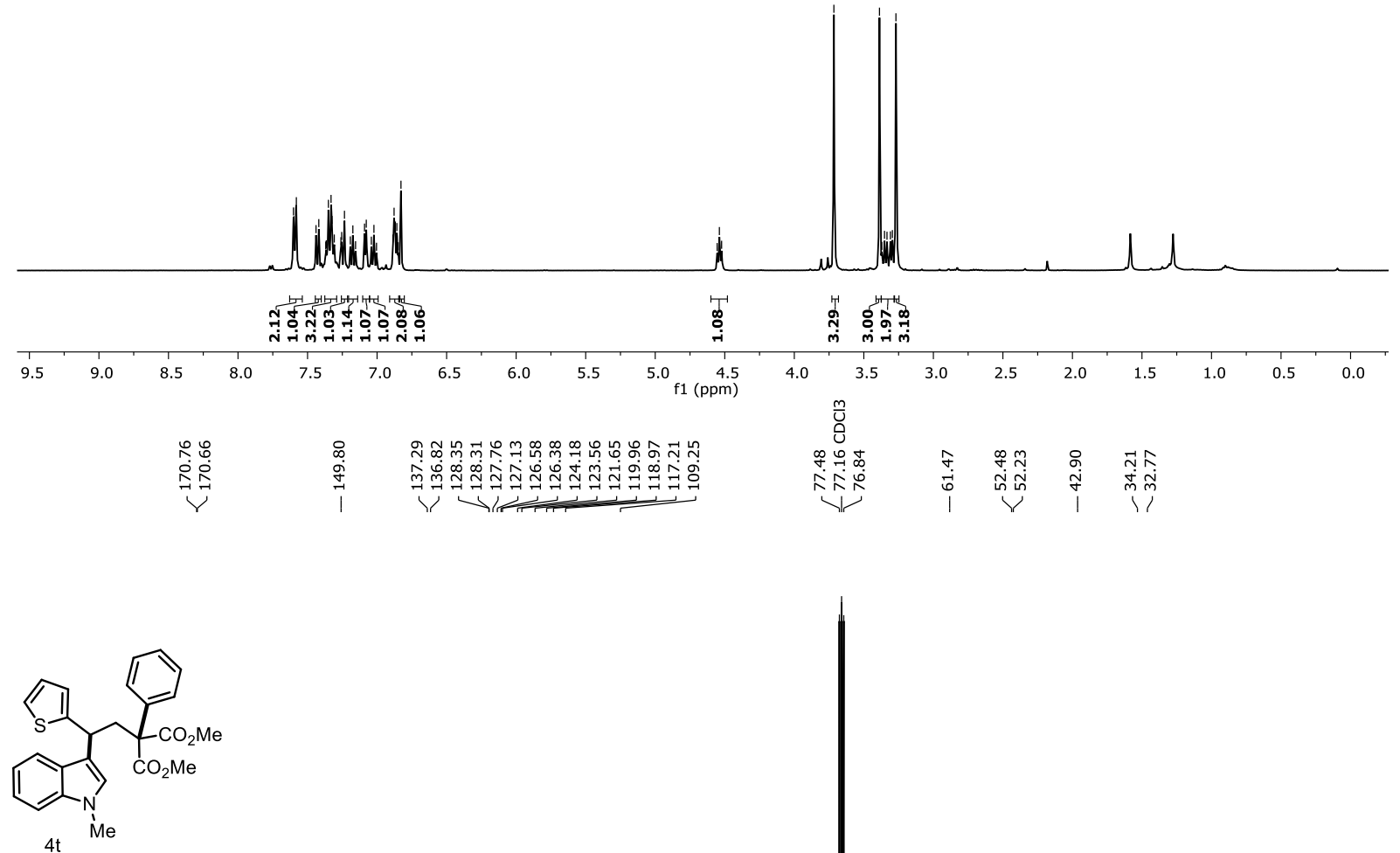

(100 MHz, $\mathrm{CDCl}_{3}$ )

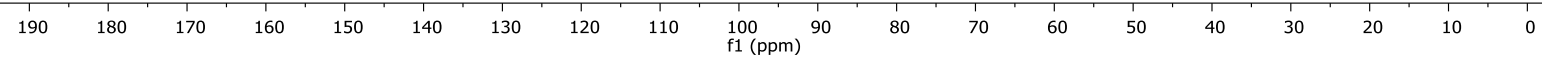




\section{苔}

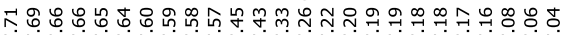

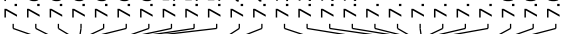
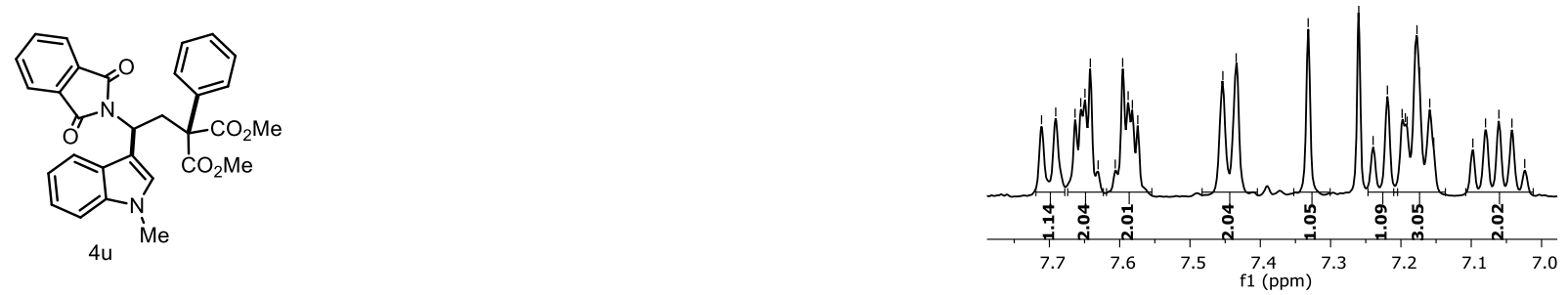

$\left(400 \mathrm{MHz}, \mathrm{CDCl}_{3}\right)$
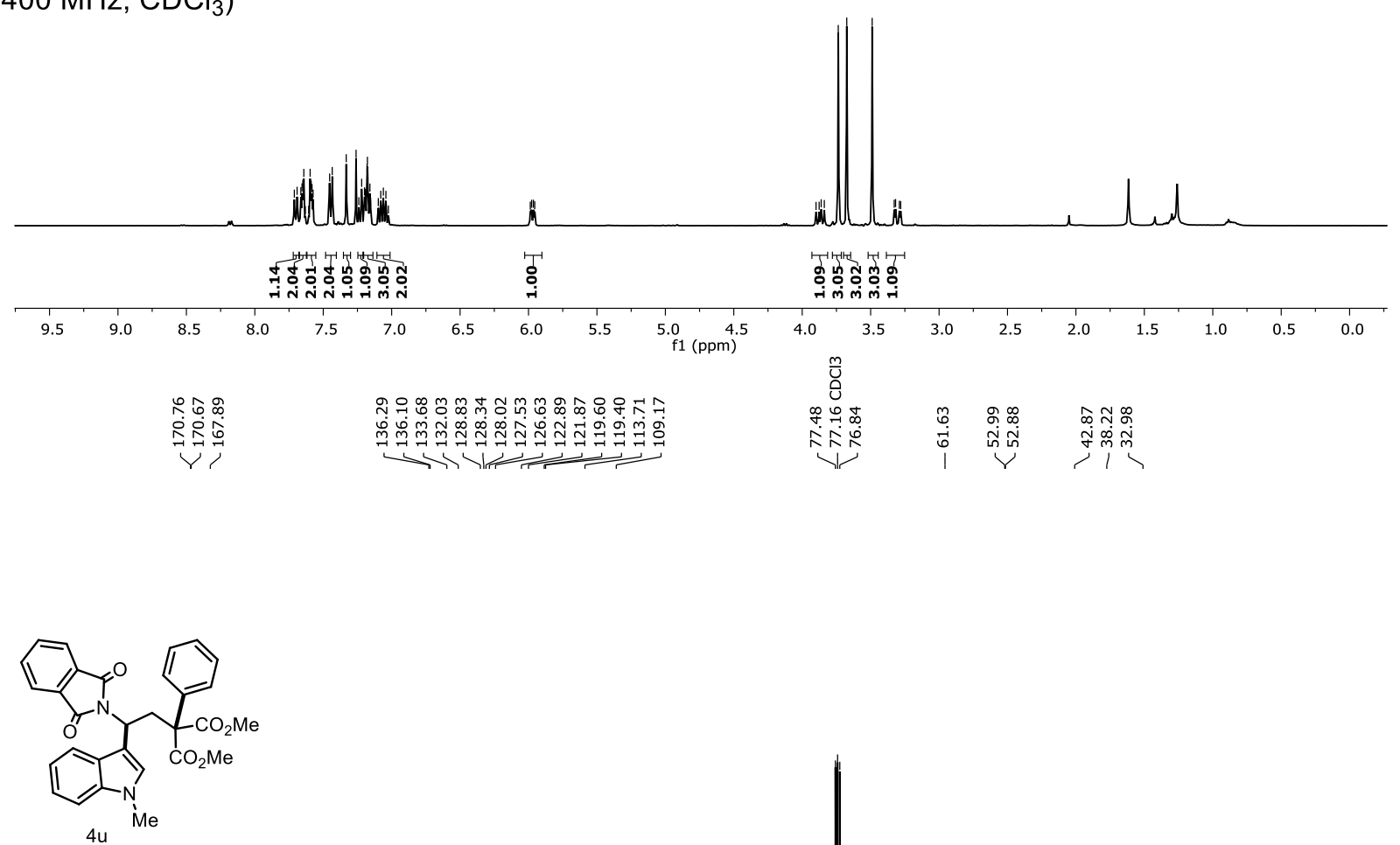

(100 MHz, $\mathrm{CDCl}_{3}$ )

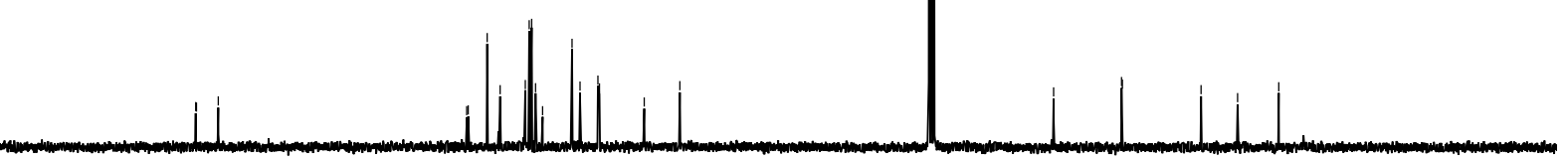

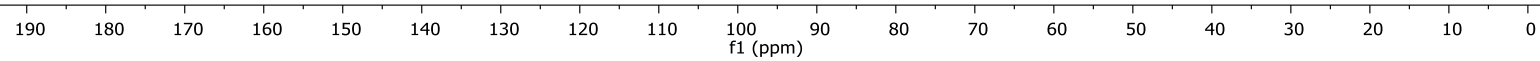




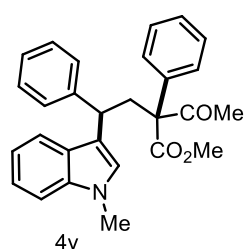

$\left(400 \mathrm{MHz}, \mathrm{CDCl}_{3}\right)$

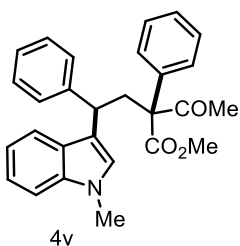

$\left(100 \mathrm{MHz}, \mathrm{CDCl}_{3}\right)$

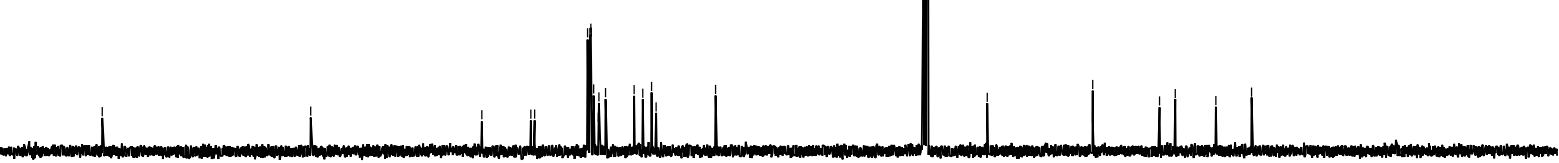

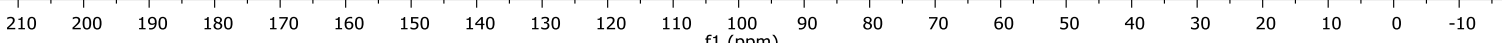


<smiles>COC(=O)C(C)(CC(c1ccc(OC)cc1)c1cn(C)c2ccc(O)cc12)c1ccccc1</smiles>

5 a

$\left(400 \mathrm{MHz} \mathrm{CDCl}_{3}\right)$
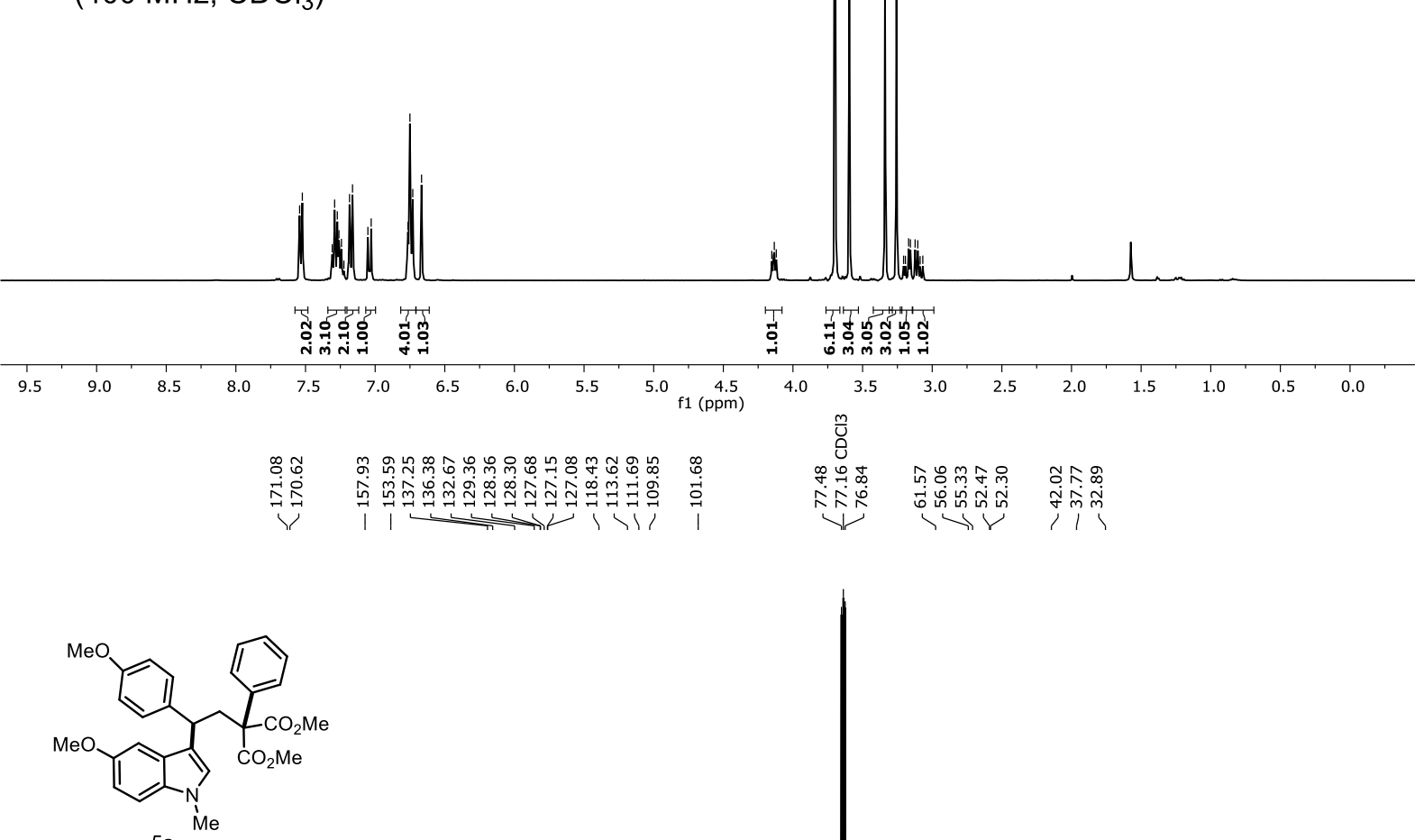

$5 a$

$\left(100 \mathrm{MHz}, \mathrm{CDCl}_{3}\right)$

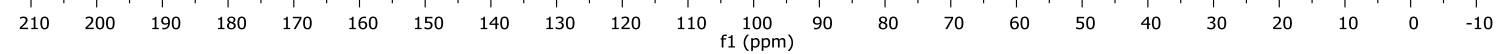




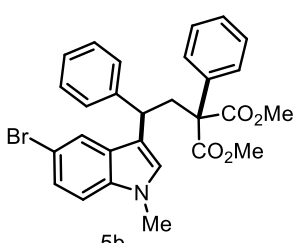

$5 b^{\mathrm{Me}}$

(400 MHz, $\mathrm{CDCl}_{3}$ )

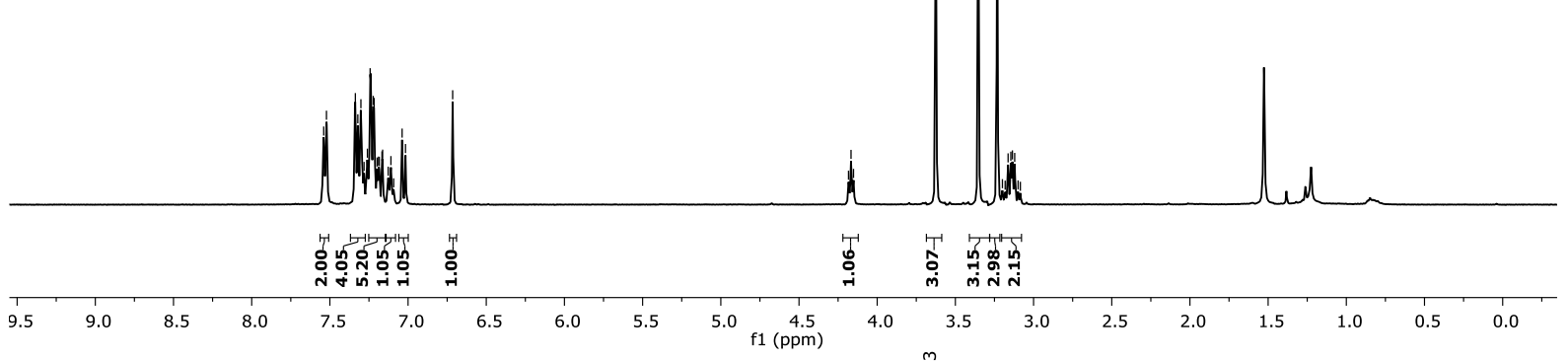

V)

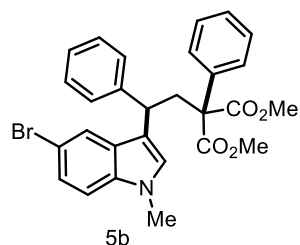

(100 MHz, $\mathrm{CDCl}_{3}$ )

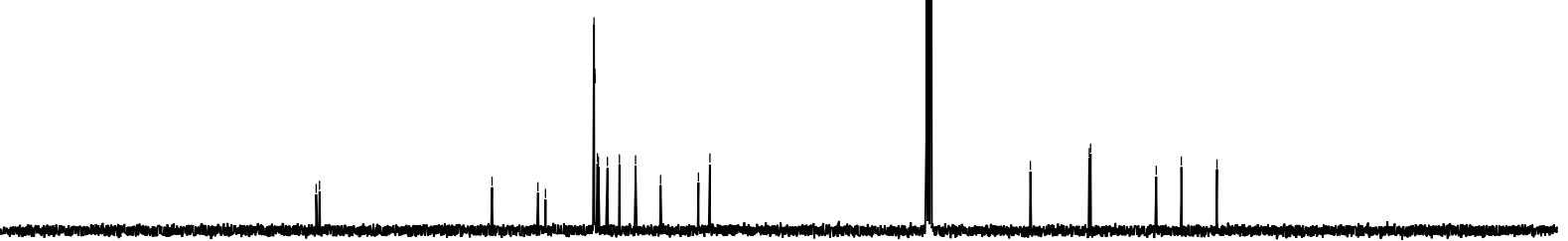

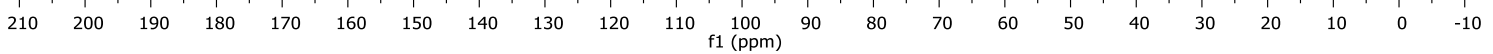




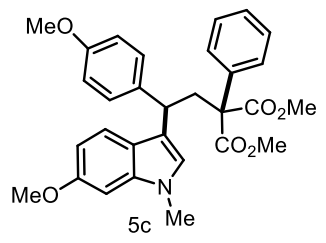

(400 MHz, $\mathrm{CDCl}_{3}$ )
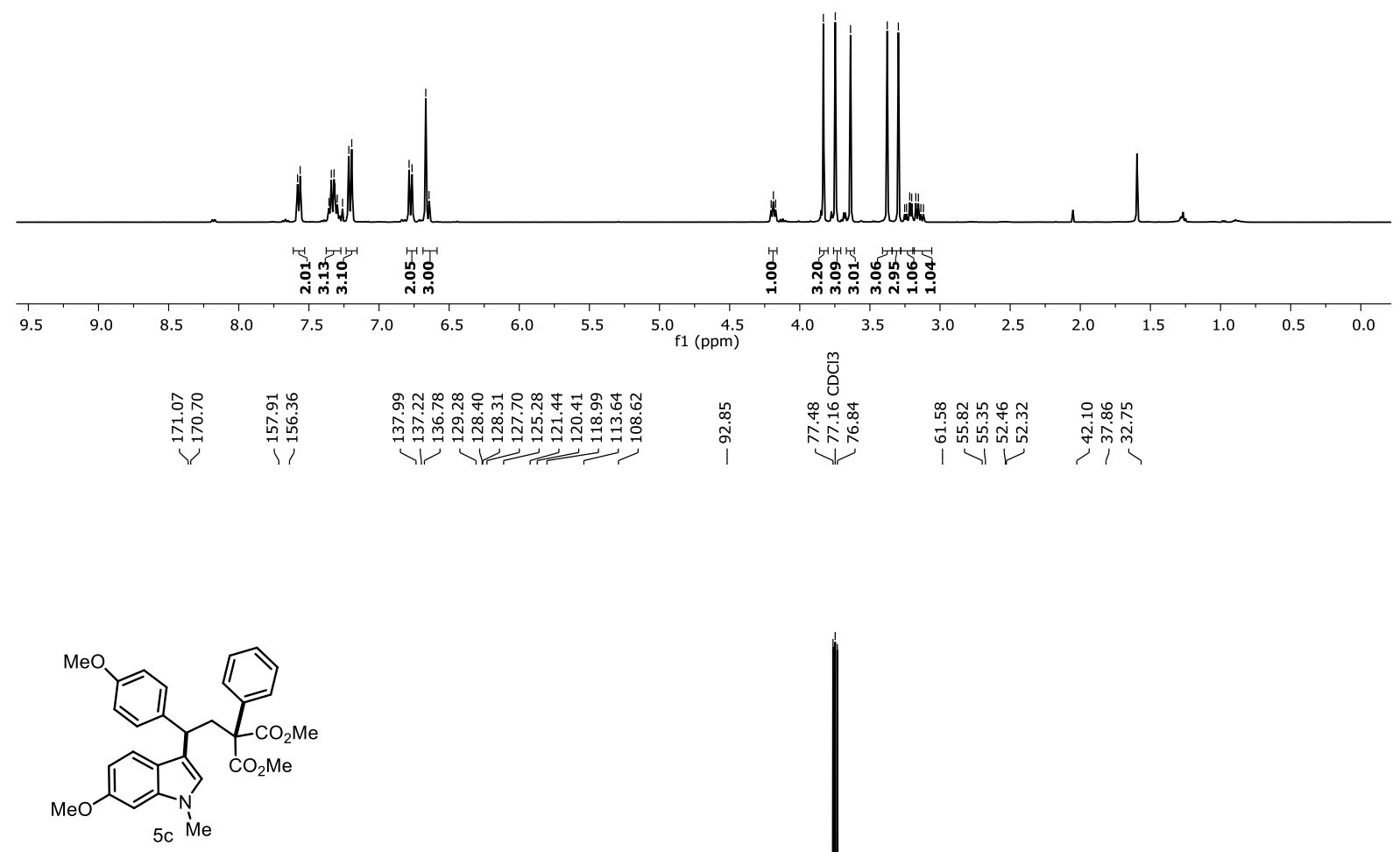

$\left(100 \mathrm{MHz}, \mathrm{CDCl}_{3}\right)$

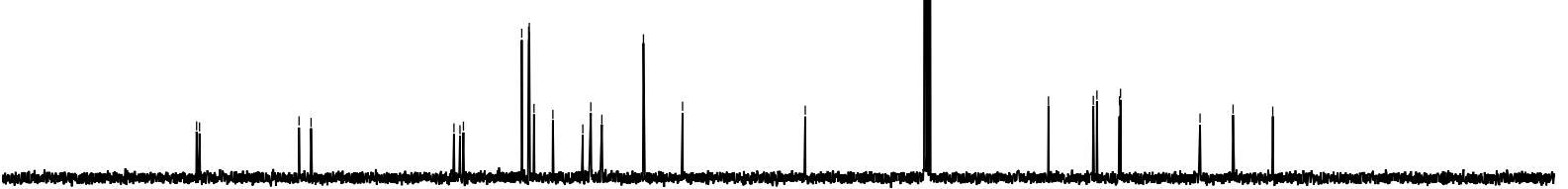

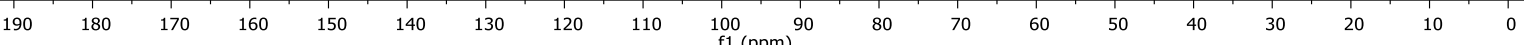



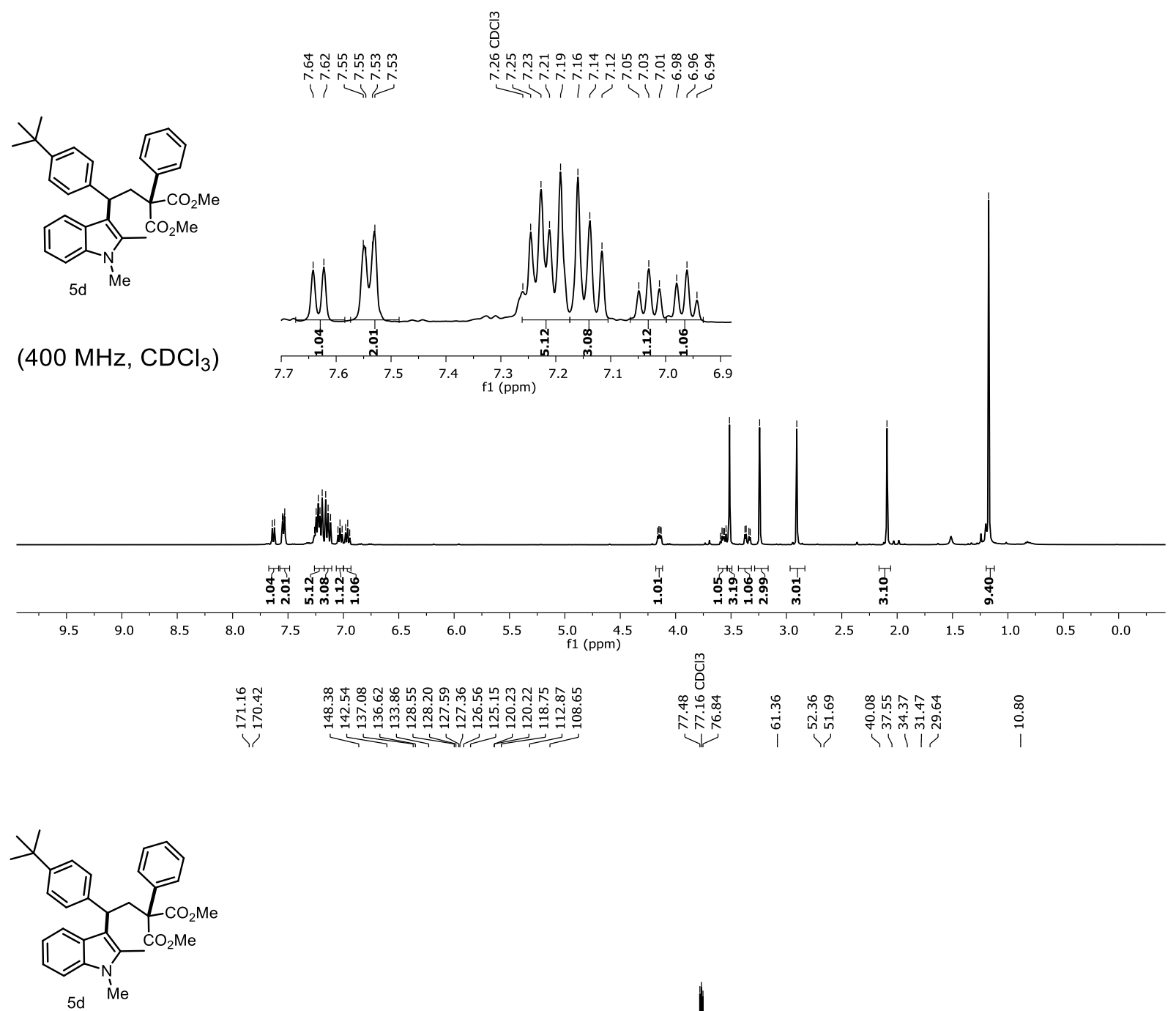

(100 MHz, $\mathrm{CDCl}_{3}$ )

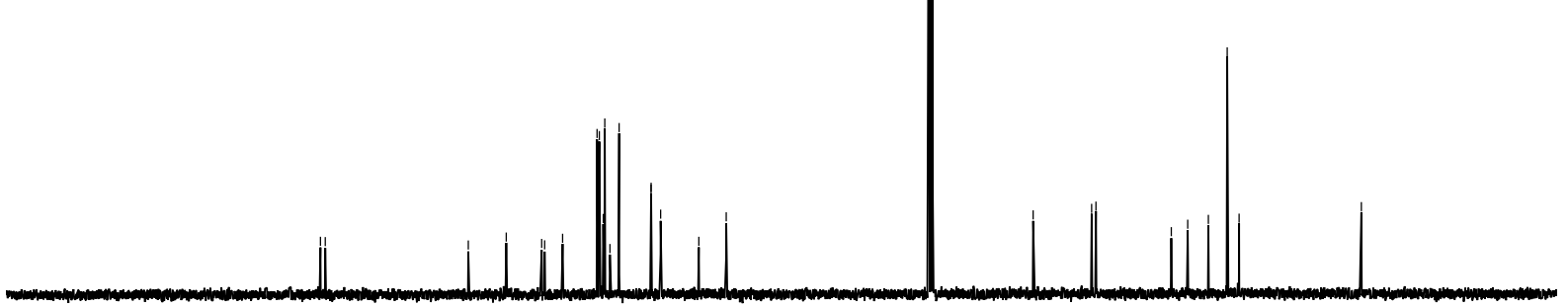

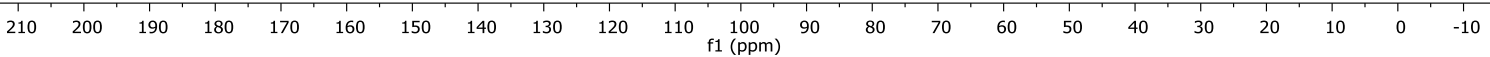




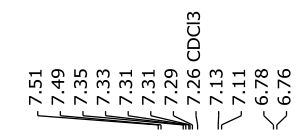

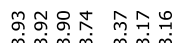

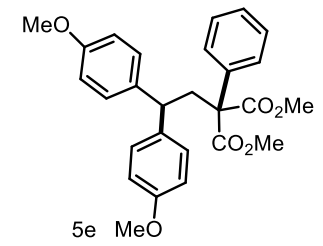

(400 MHz, $\mathrm{CDCl}_{3}$ )

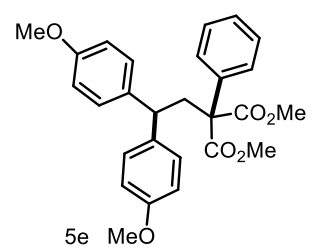

$\left(100 \mathrm{MHz}, \mathrm{CDCl}_{3}\right)$

\begin{tabular}{|c|c|c|c|}
\hline 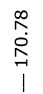 & \begin{tabular}{l}
$\hat{a}$ \\
$\hat{n}$ \\
\multirow{1}{*}{}
\end{tabular} & 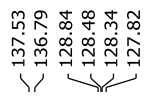 & 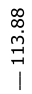 \\
\hline
\end{tabular}

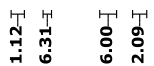

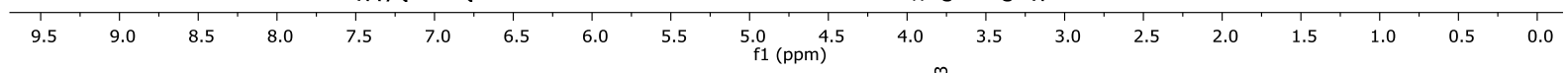

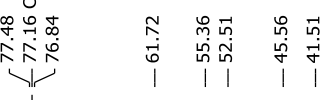

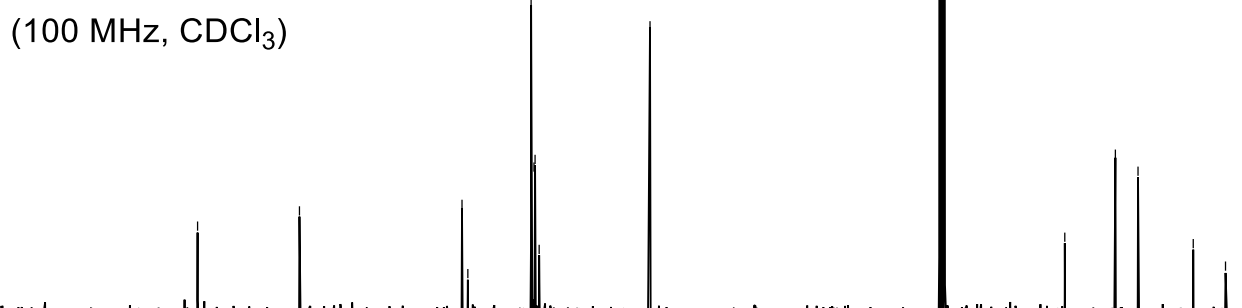

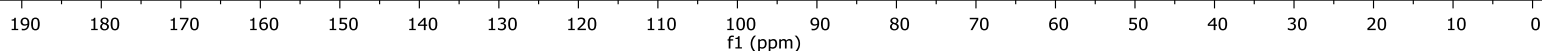


<smiles>COc1ccc(C)cc1CC(CC(OC)(C(C)=O)c1ccccc1)c1ccc(O)cc1</smiles>

$5 f$
造

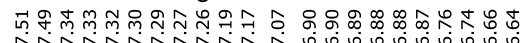

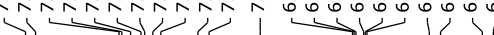

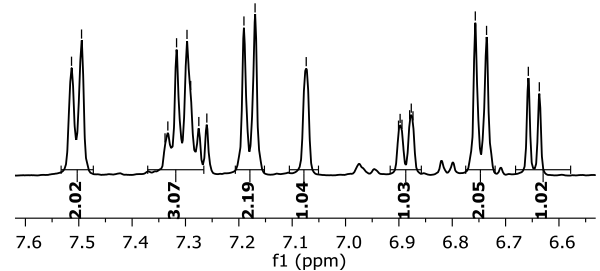

(400 MHz, $\mathrm{CDCl}_{3}$ )

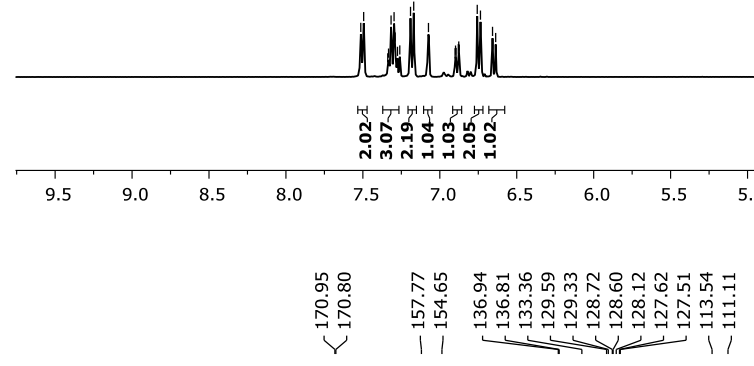<smiles>COc1ccc(C(CC(C)(c2ccccc2)C(C)(C)C)c2cc([N+](=O)[O-])ccc2OC)cc1</smiles>

$5 f$

$\left(100 \mathrm{MHz}, \mathrm{CDCl}_{3}\right)$

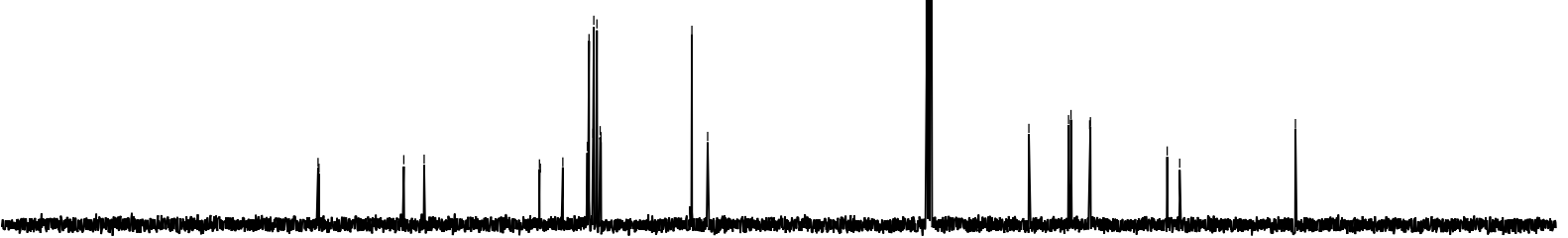

\begin{tabular}{llllllllllllllllllllllll}
\hline & 210 & 200 & 190 & 180 & 170 & 160 & 150 & 140 & 130 & 120 & 110 & $\begin{array}{c}100 \\
\mathrm{f} 1(\mathrm{ppm})\end{array}$ & 90 & 80 & 70 & 60 & 50 & 40 & 30 & 20 & 10 & 0 & -10
\end{tabular} 


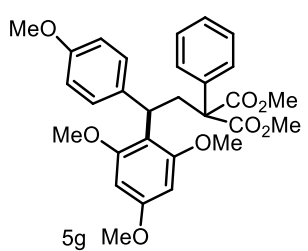

(400 MHz, $\mathrm{CDCl}_{3}$ )

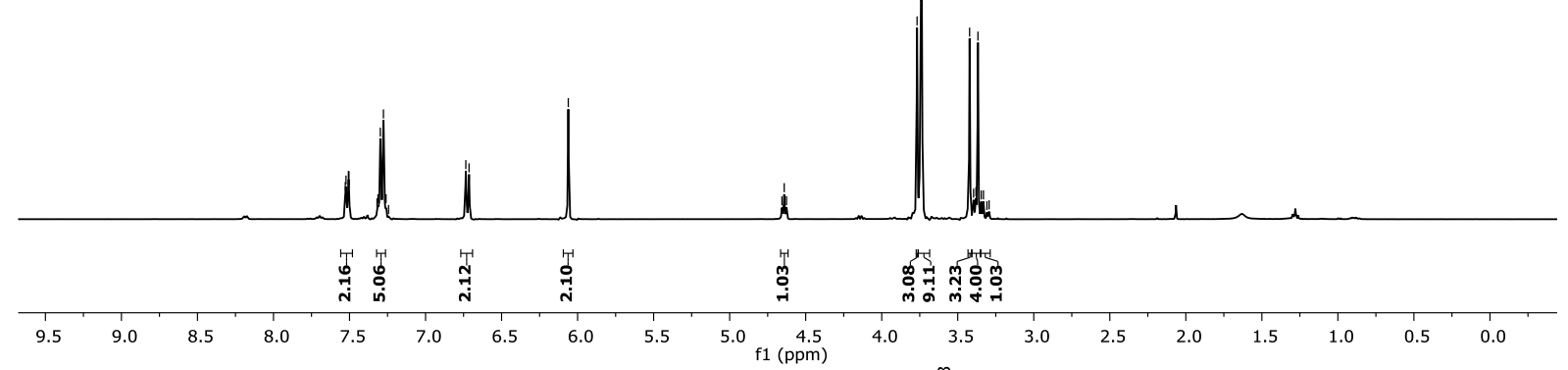

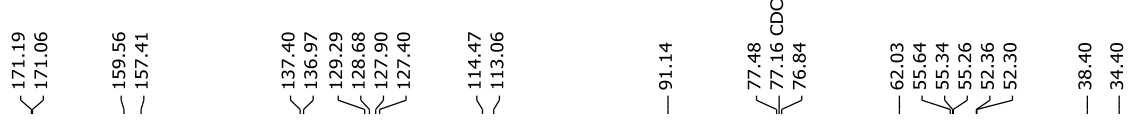

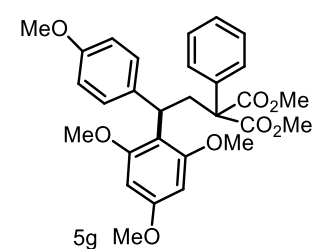

(100 MHz, $\mathrm{CDCl}_{3}$ )

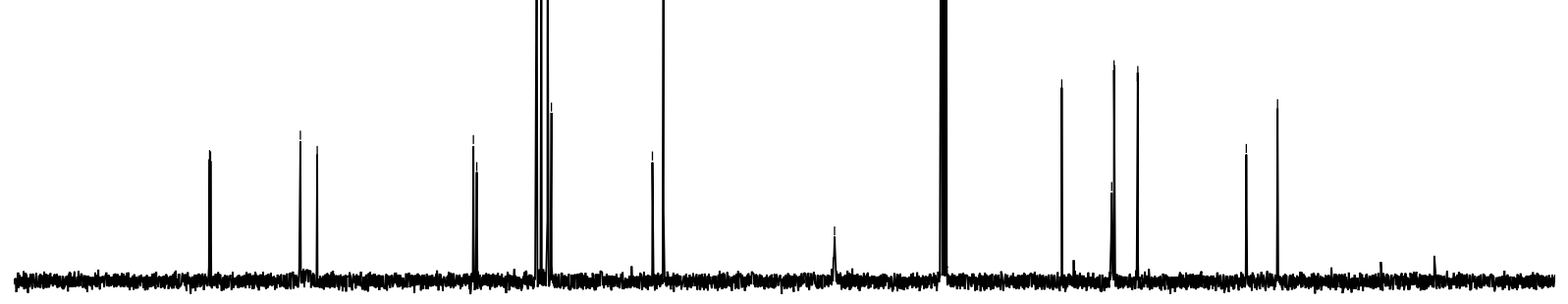

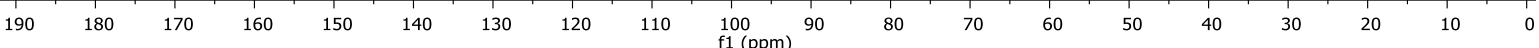




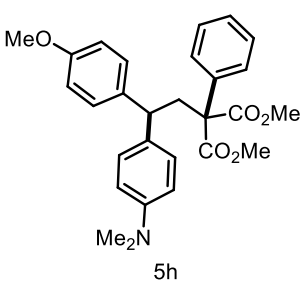

(400 MHz, $\mathrm{CDCl}_{3}$ )

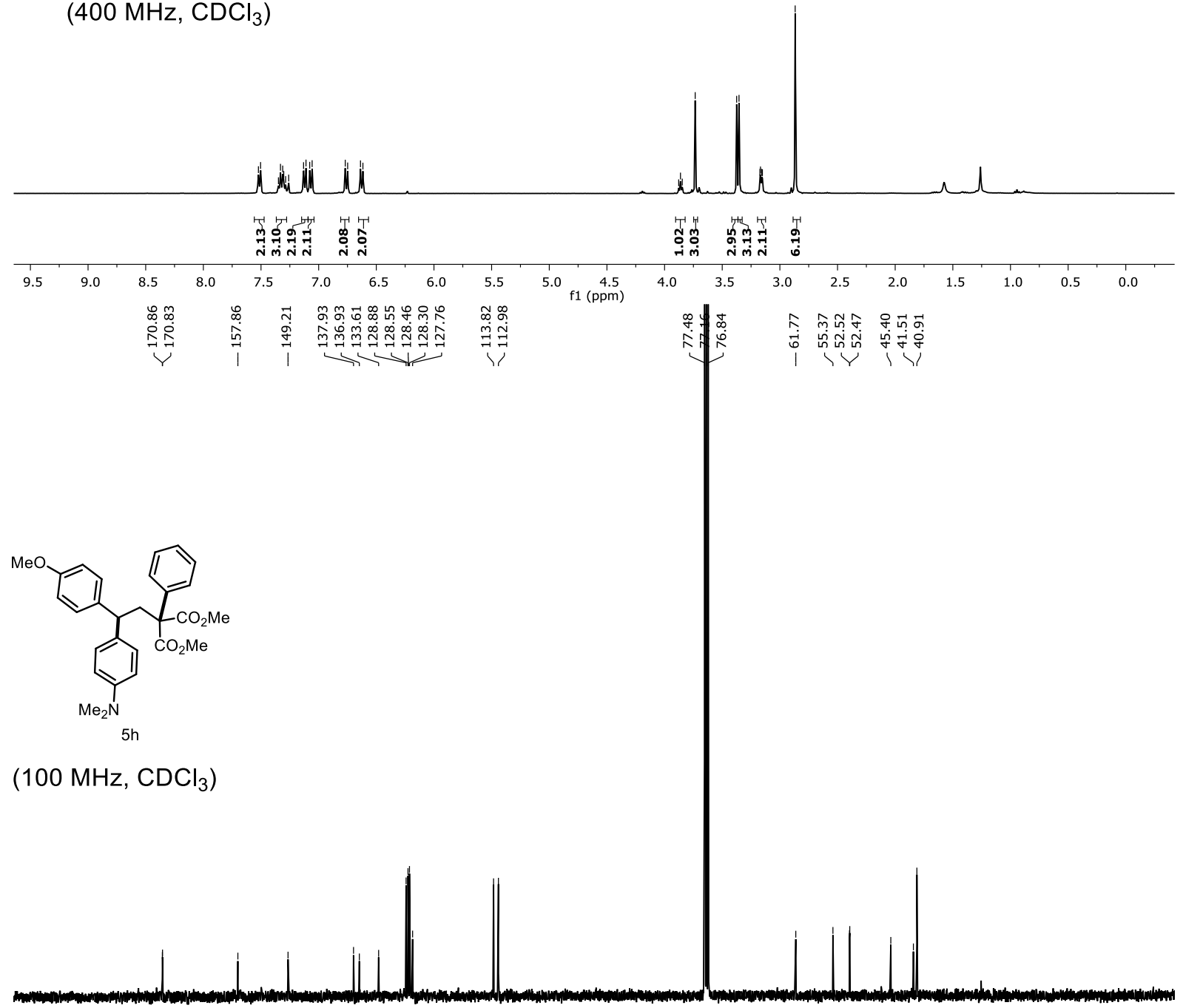

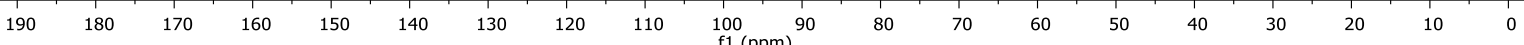




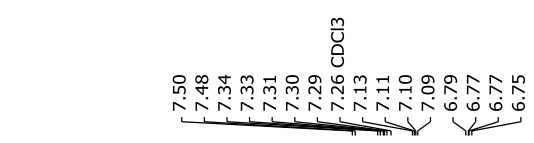

요요

i்m

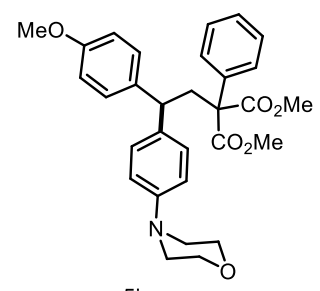

(400 MHz, $\mathrm{CDCl}_{3}$ )

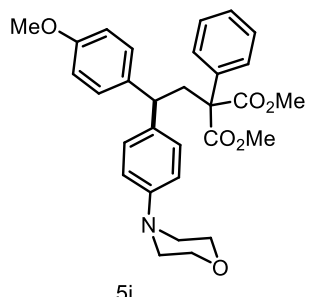

$\left(100 \mathrm{MHz}, \mathrm{CDCl}_{3}\right)$

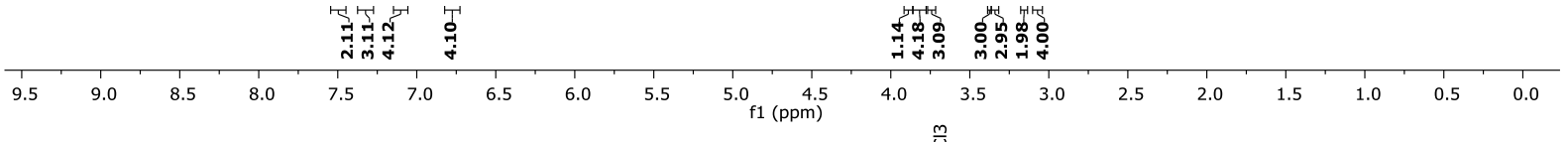

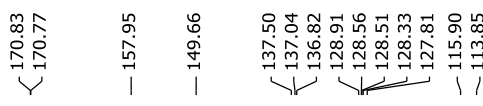

8

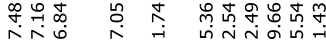

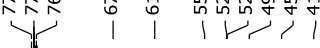

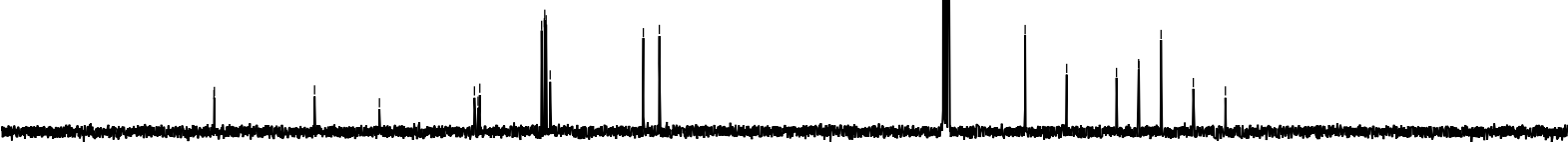

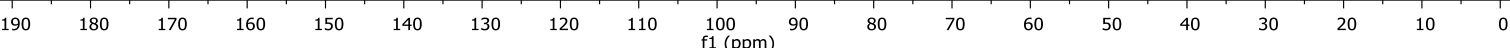


<smiles>COc1ccc(C(CC(OC(C)(O)C(C)(O)c2ccc(C)cc2)(c2ccccc2)c2ccccc2)C(C)(C)O)cc1</smiles>

5

(400 MHz, $\mathrm{CDCl}_{3}$ )
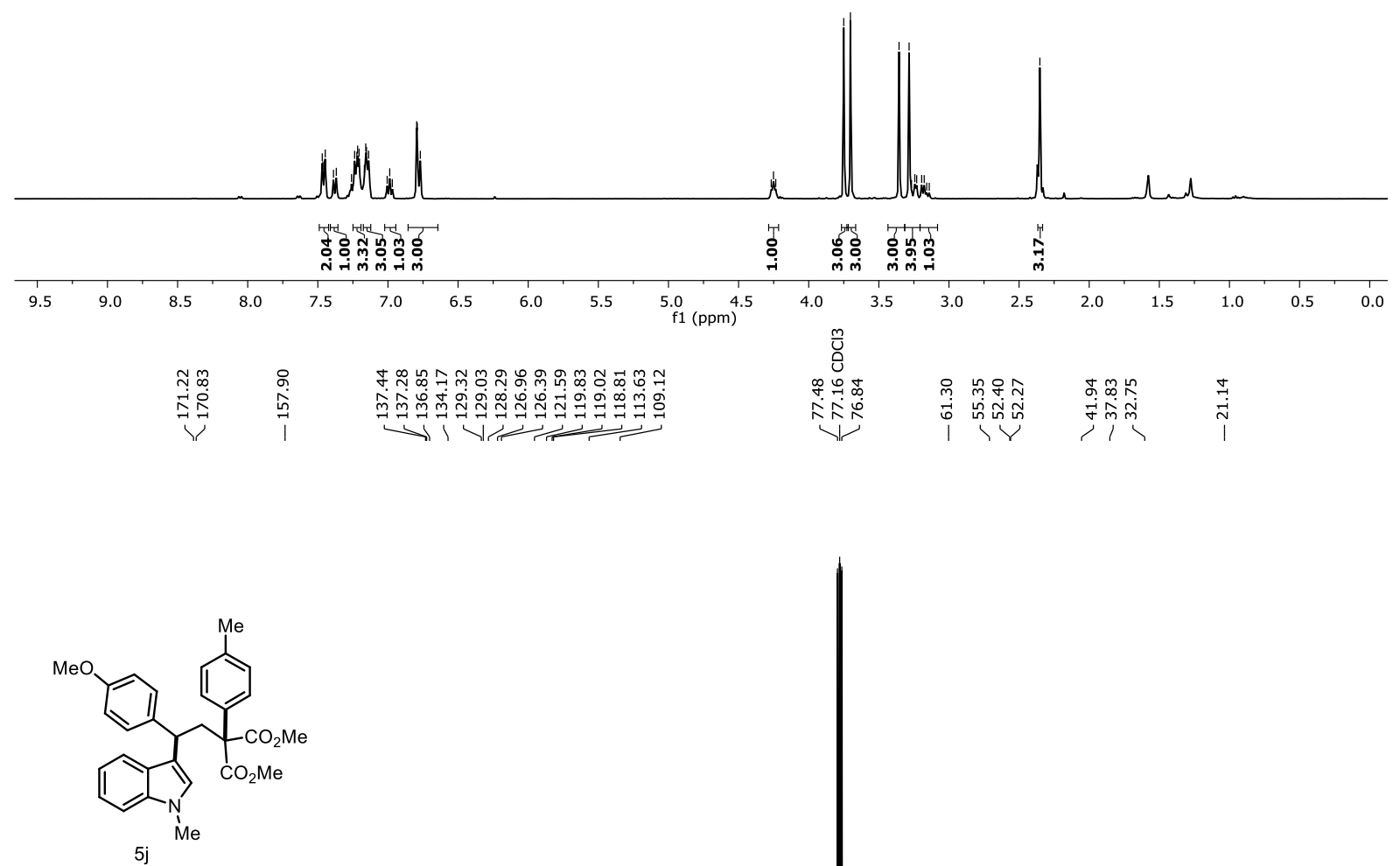

(100 MHz, $\mathrm{CDCl}_{3}$ )

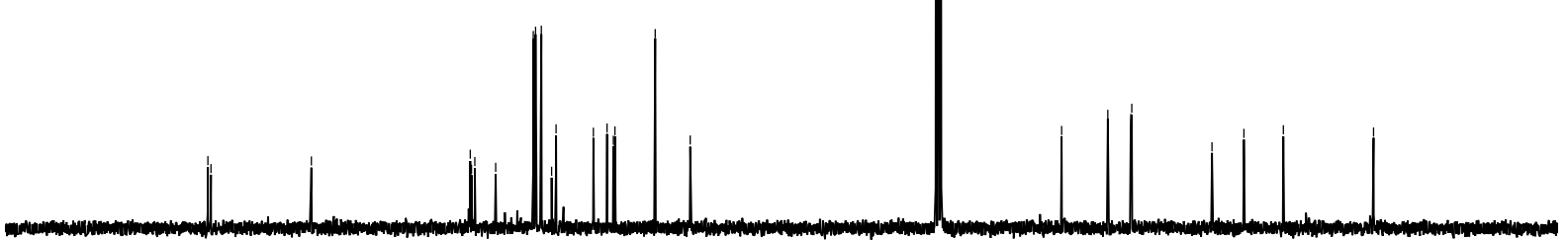

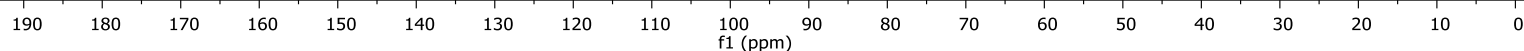




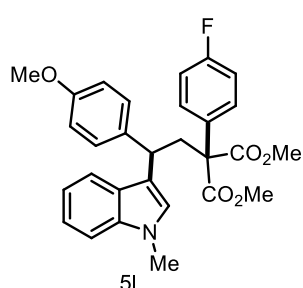

(400 MHz, $\mathrm{CDCl}_{3}$ )

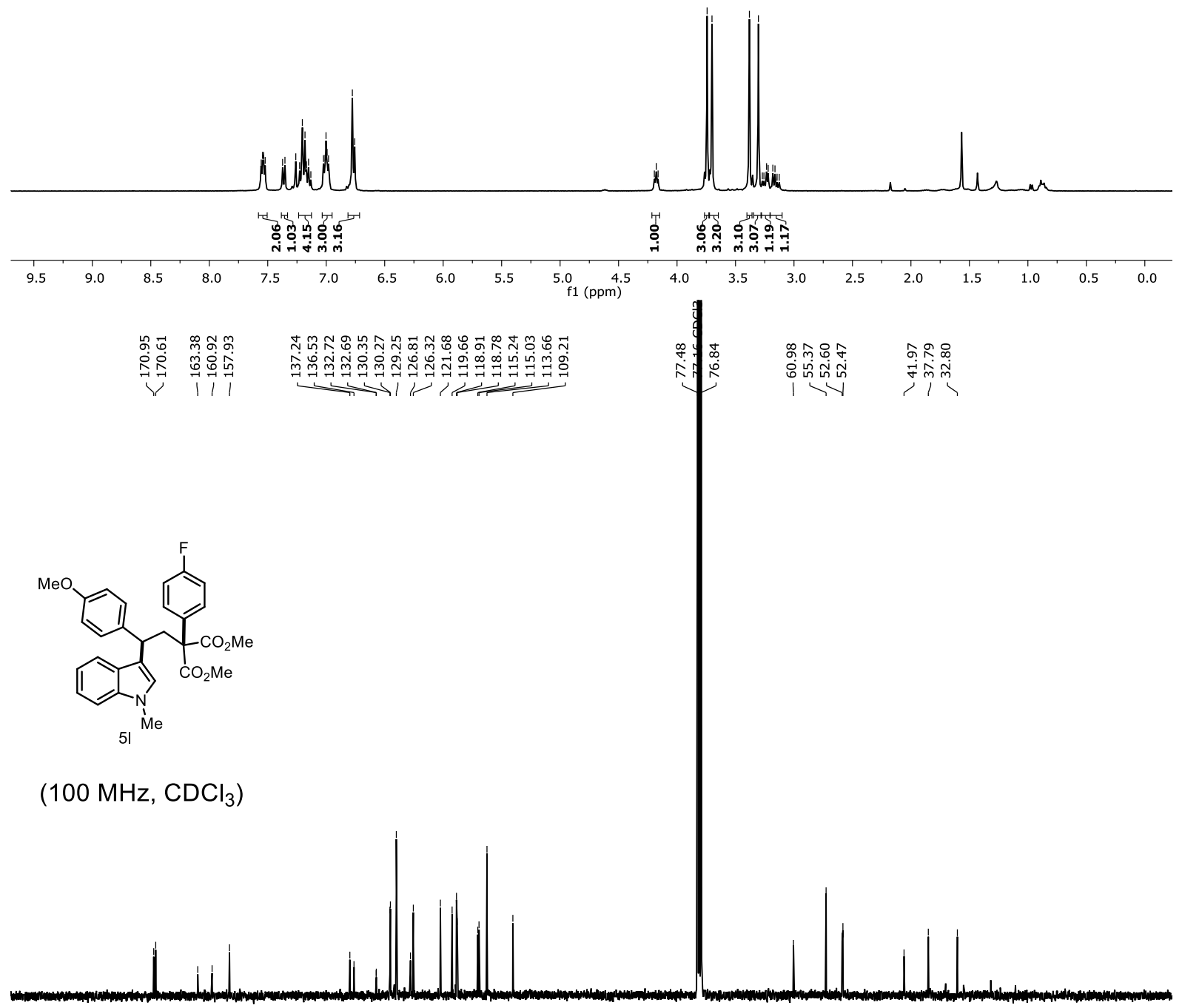

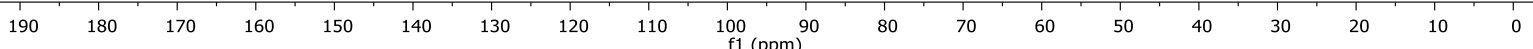




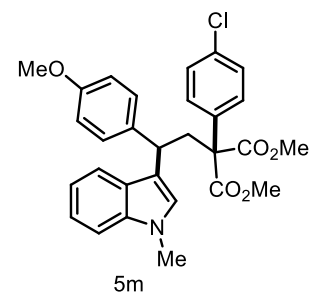

(400 MHz, $\mathrm{CDCl}_{3}$ )

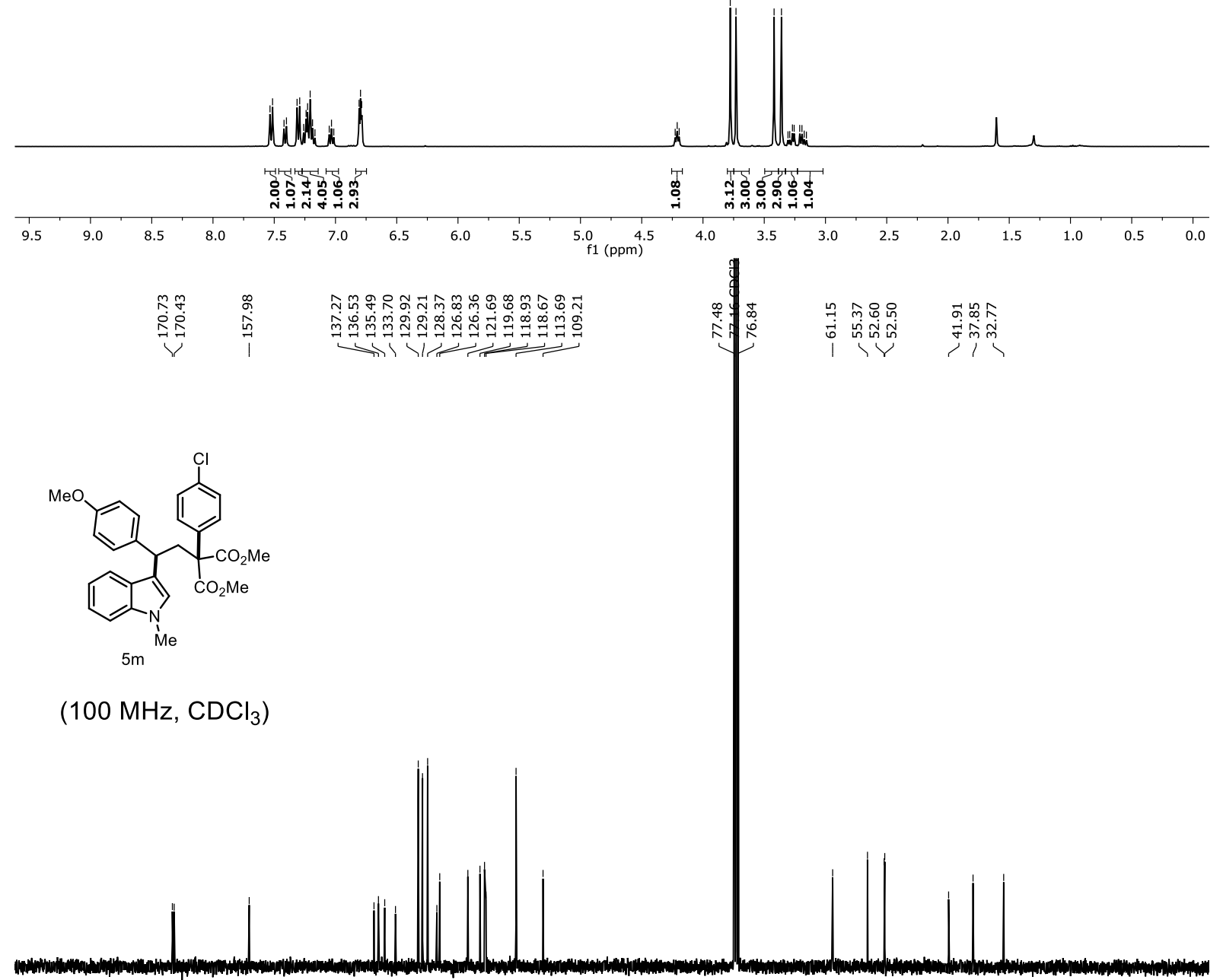

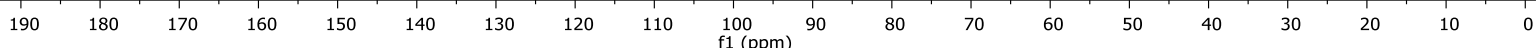




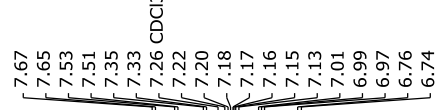

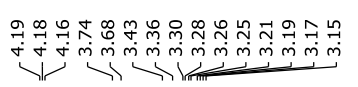

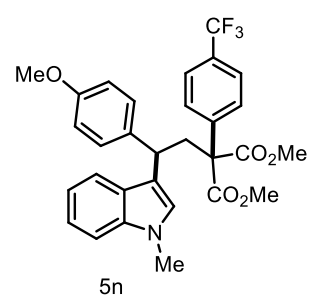

(400 MHz, $\mathrm{CDCl}_{3}$ )
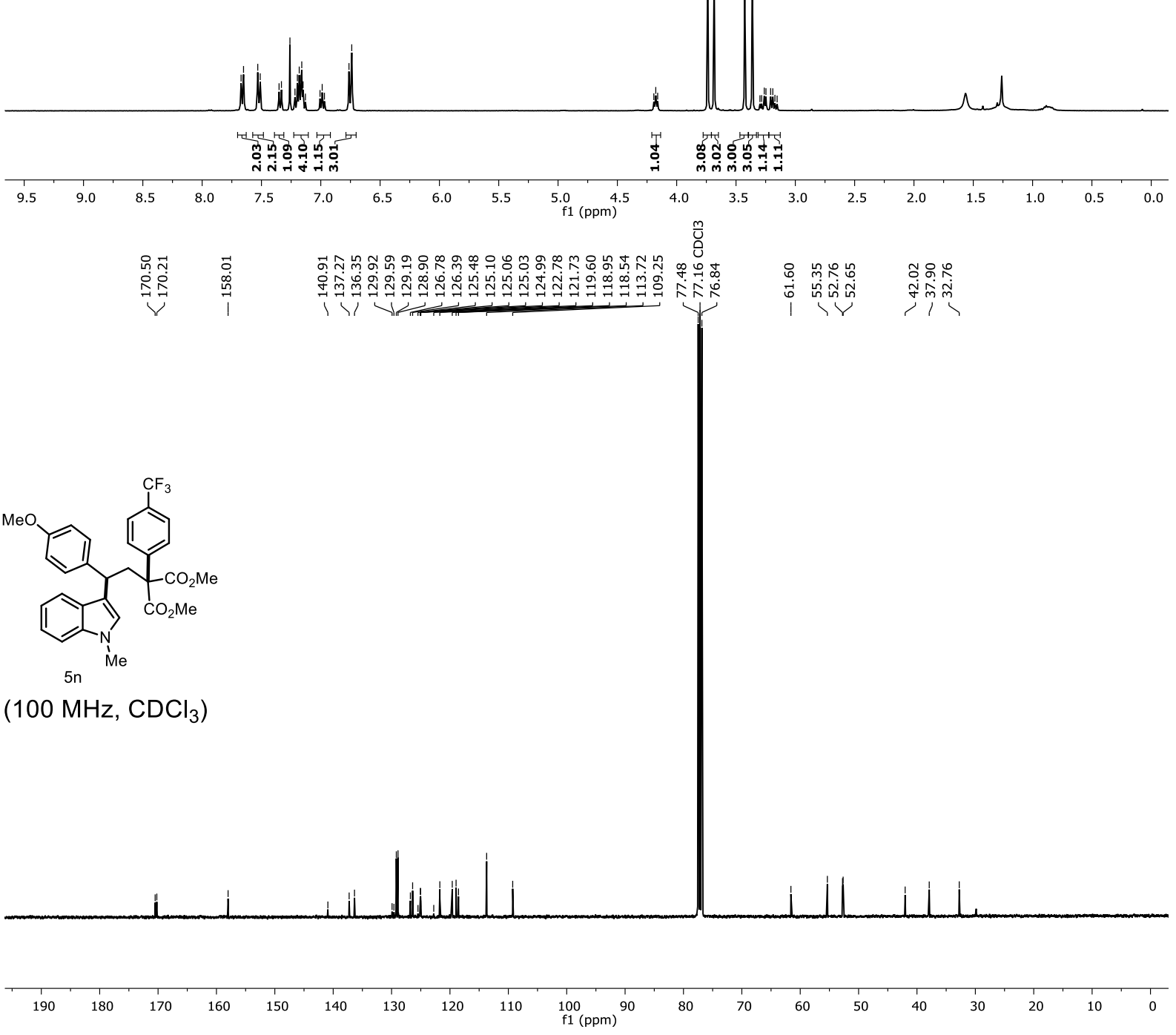

72 


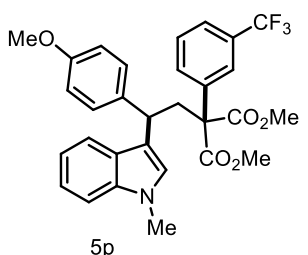

$5 p$

(400 $\mathrm{MHz}, \mathrm{CDCl}_{3}$ )
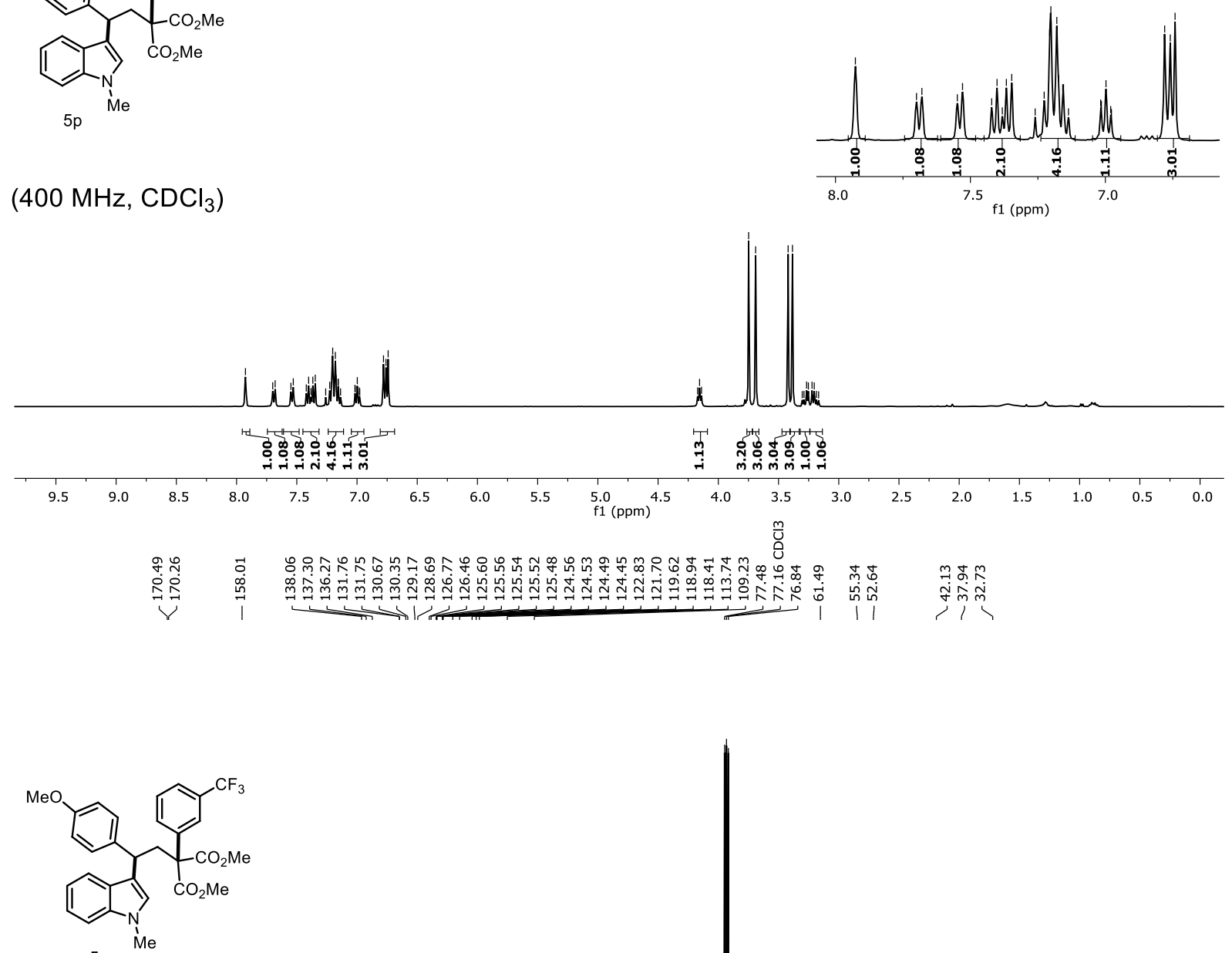

$5 p$

$\left(100 \mathrm{MHz}, \mathrm{CDCl}_{3}\right)$

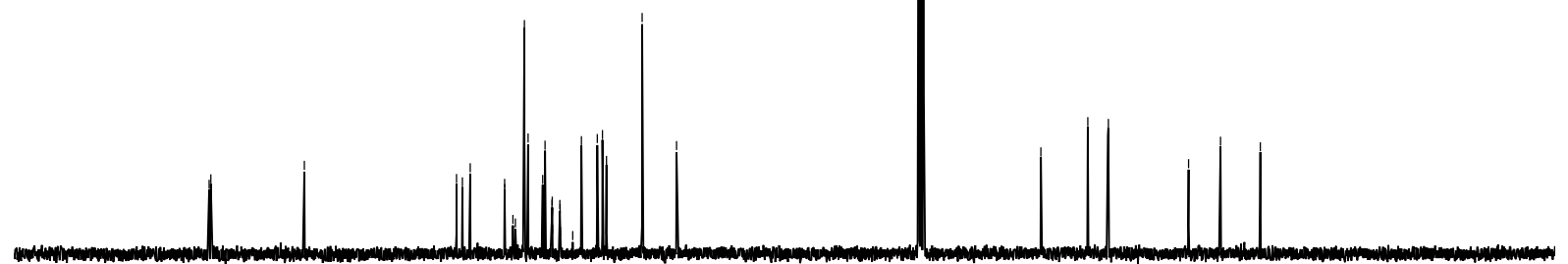

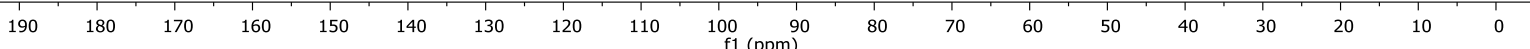




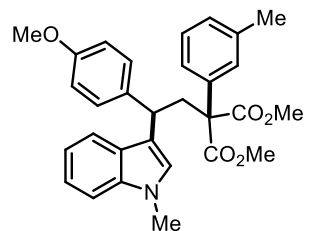

$5 \mathrm{q}$

(400 MHz, $\mathrm{CDCl}_{3}$ )

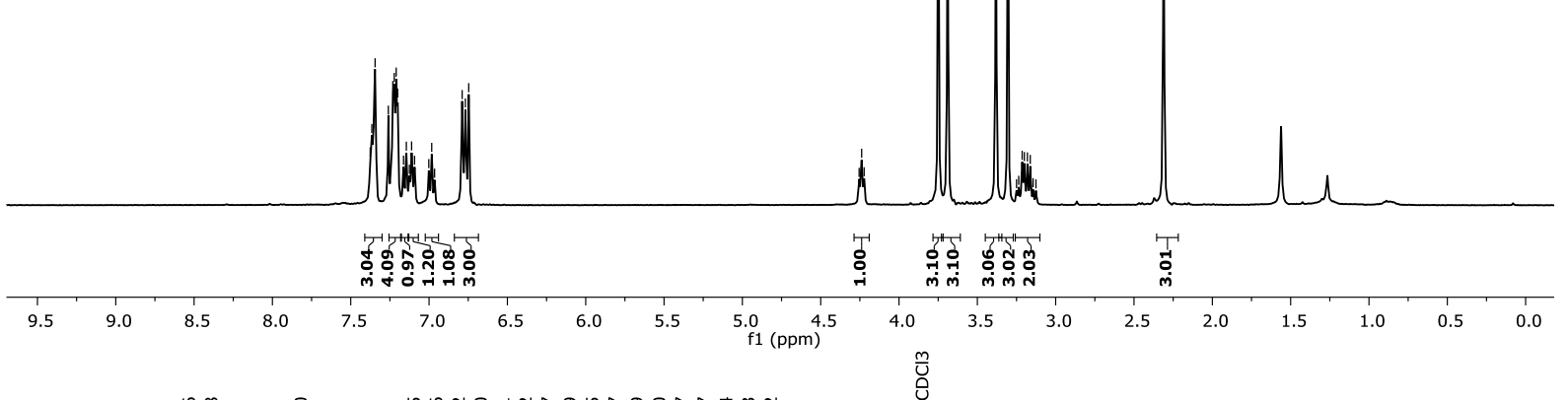

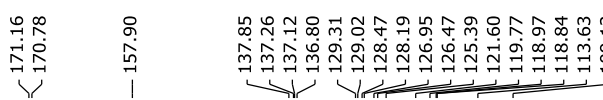

诲

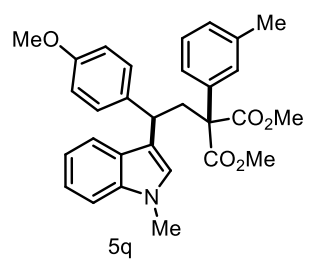

$\left(100 \mathrm{MHz}, \mathrm{CDCl}_{3}\right)$

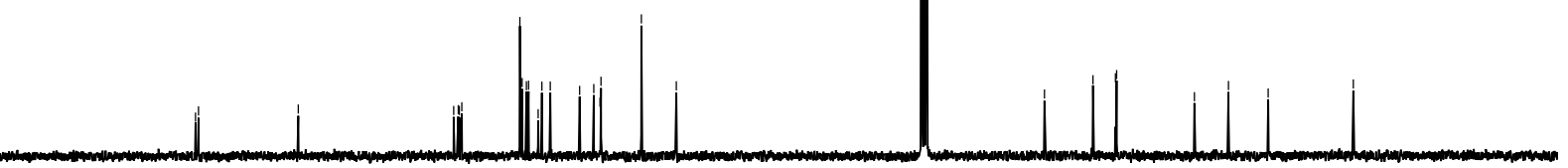

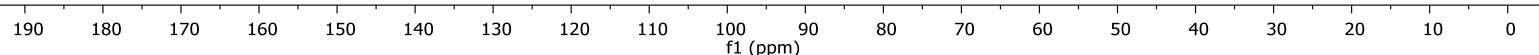




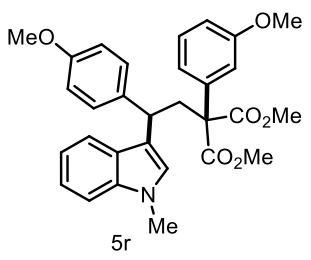

$\left(400 \mathrm{MHz}, \mathrm{CDCl}_{3}\right)$
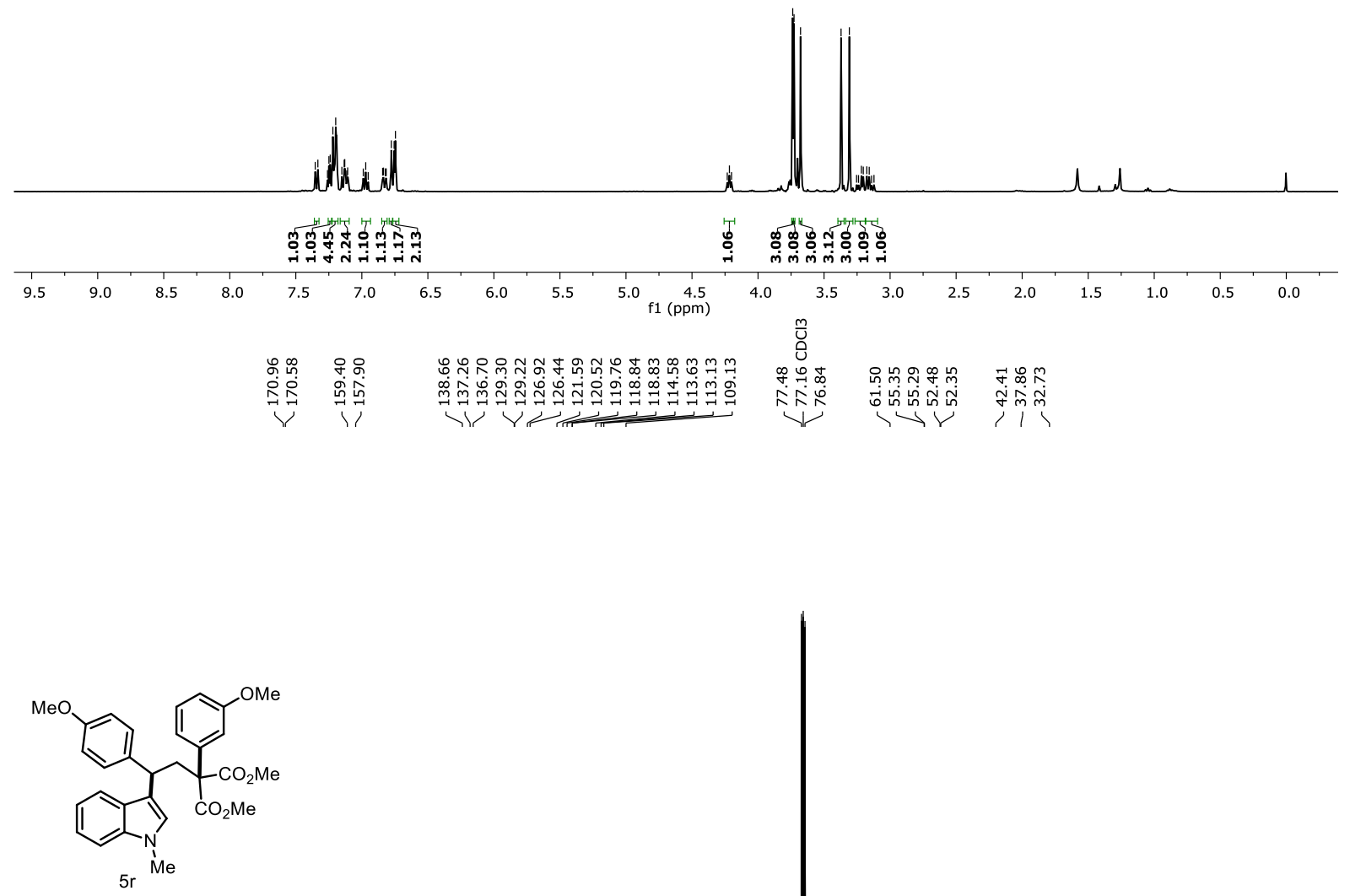

$\left(100 \mathrm{MHz}, \mathrm{CDCl}_{3}\right)$

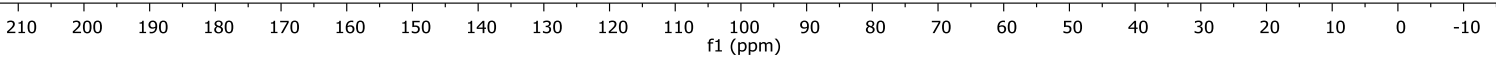


<smiles>COc1ccc(C(CC(C)(C(C)=O)c2cccc(F)c2)c2cn(C)c3ccccc23)cc1</smiles>

(400 $\mathrm{MHz}, \mathrm{CDCl}_{3}$ )
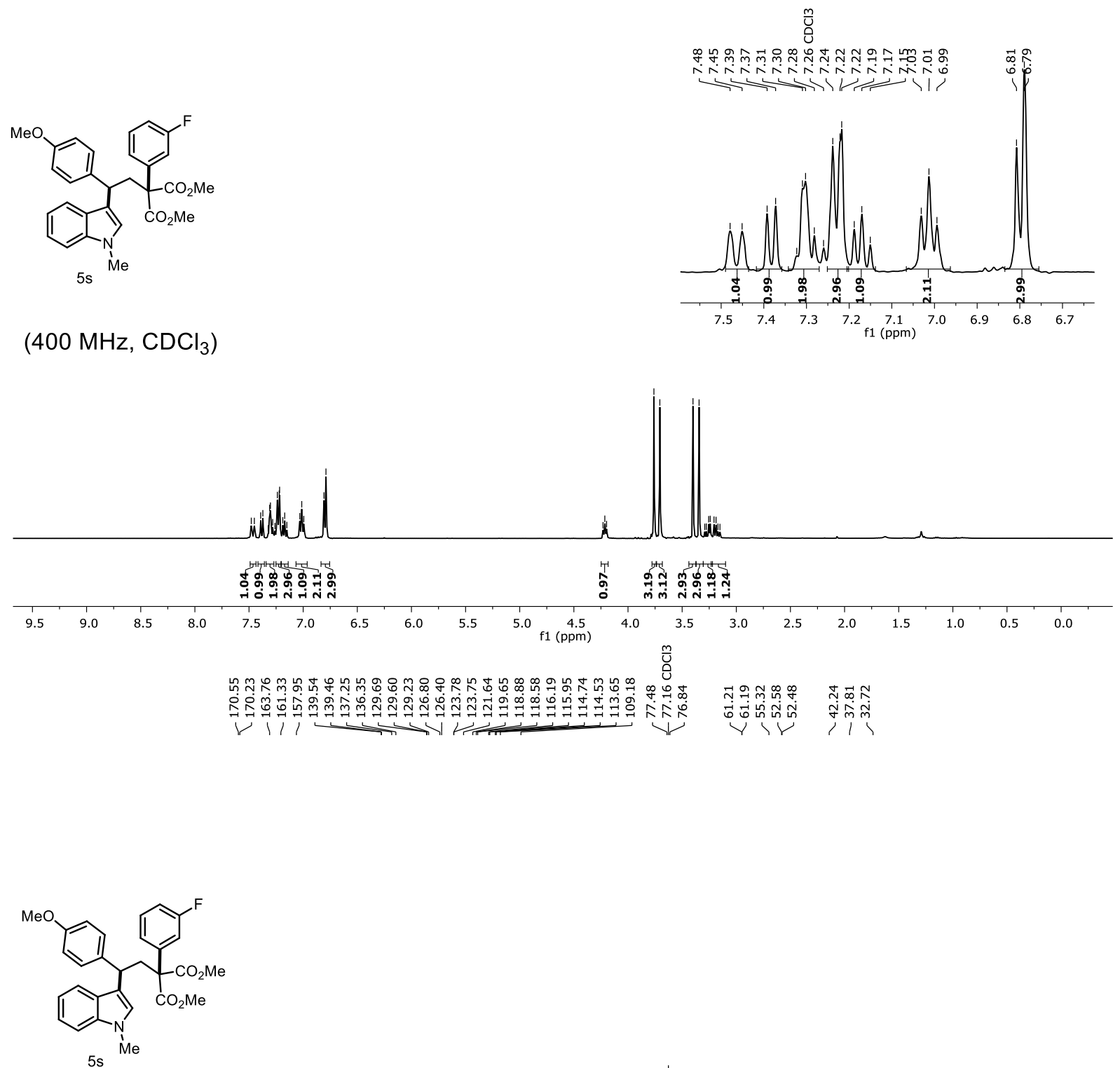

(100 MHz, $\mathrm{CDCl}_{3}$ )

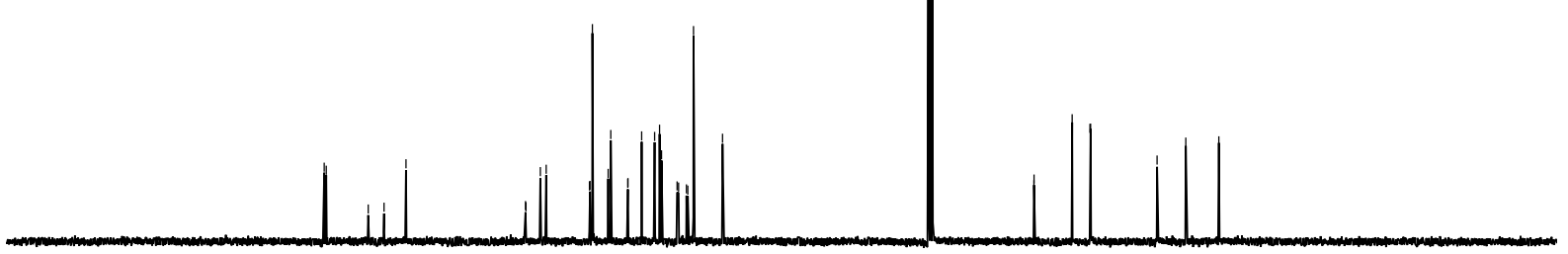

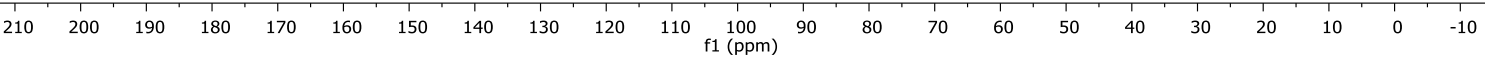




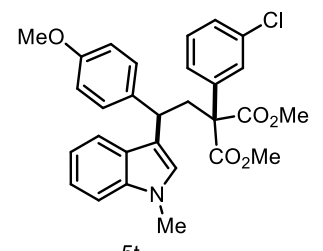

(400 MHz, $\mathrm{CDCl}_{3}$ )

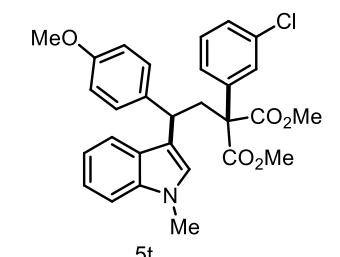

$\left(100 \mathrm{MHz}, \mathrm{CDCl}_{3}\right)$

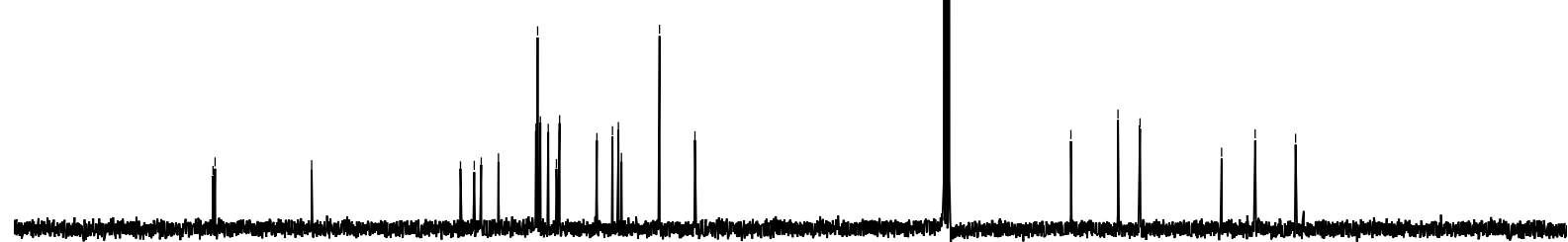

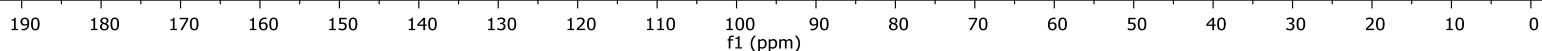




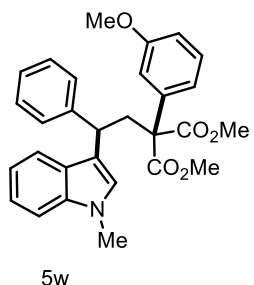

(400 MHz, $\mathrm{CDCl}_{3}$ )
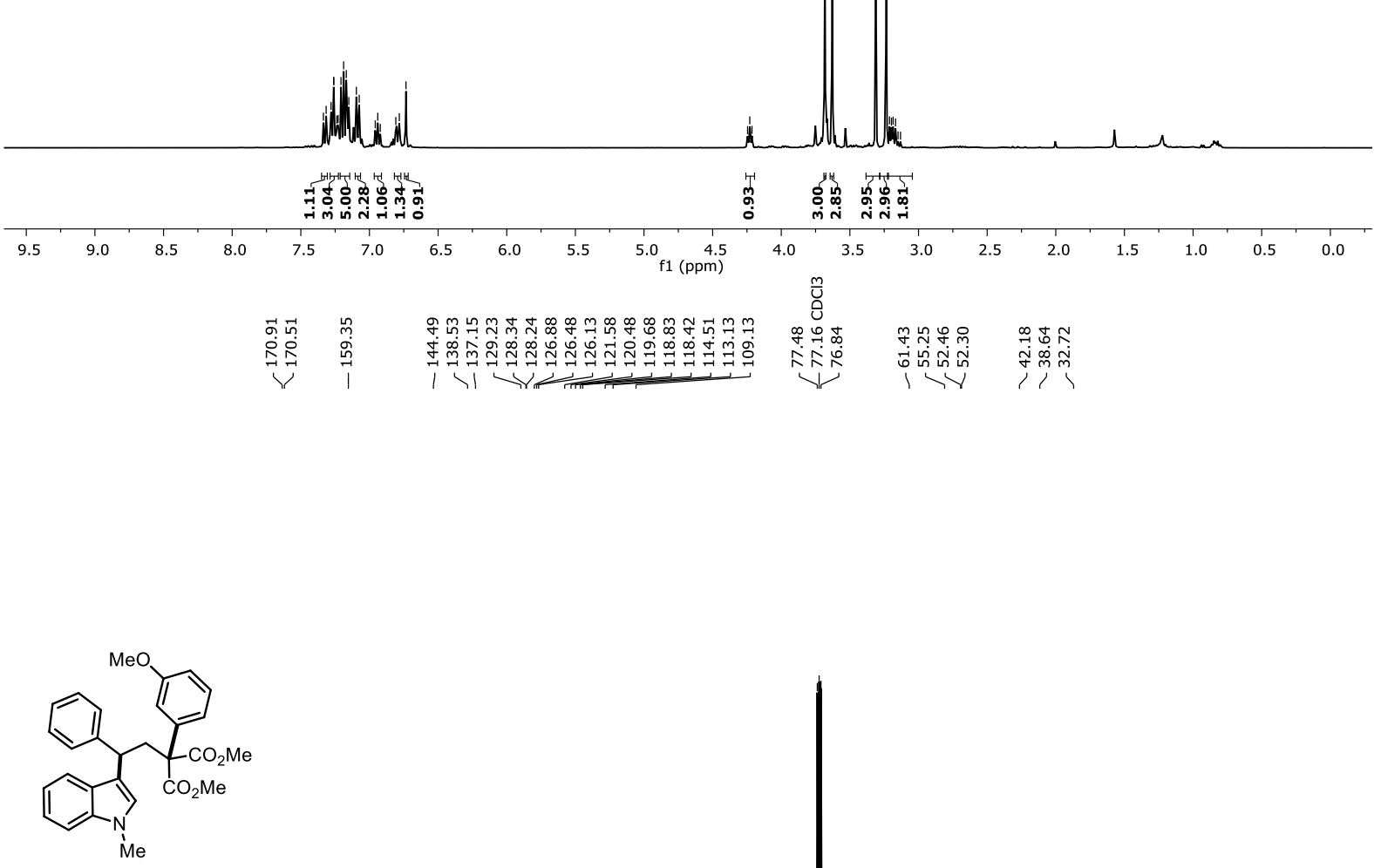

$5 w$

(100 $\mathrm{MHz}, \mathrm{CDCl}_{3}$ )

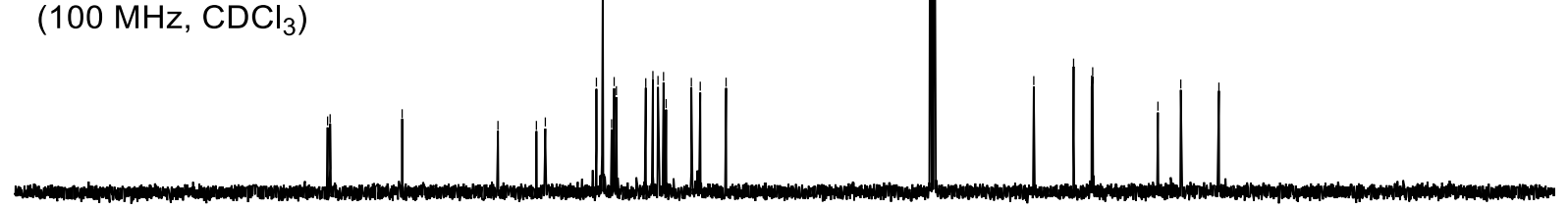

$\begin{array}{llllllllllllllllllllllll}1 & 210 & 200 & 190 & 180 & 170 & 160 & 150 & 140 & 130 & 120 & 110 & \underset{\mathrm{f} 1}{100}(\mathrm{ppm}) & 90 & 80 & 70 & 60 & 50 & 40 & 30 & 20 & 10 & 0 & -10\end{array}$ 


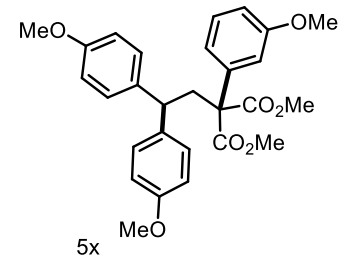

(400 MHz, $\mathrm{CDCl}_{3}$ )
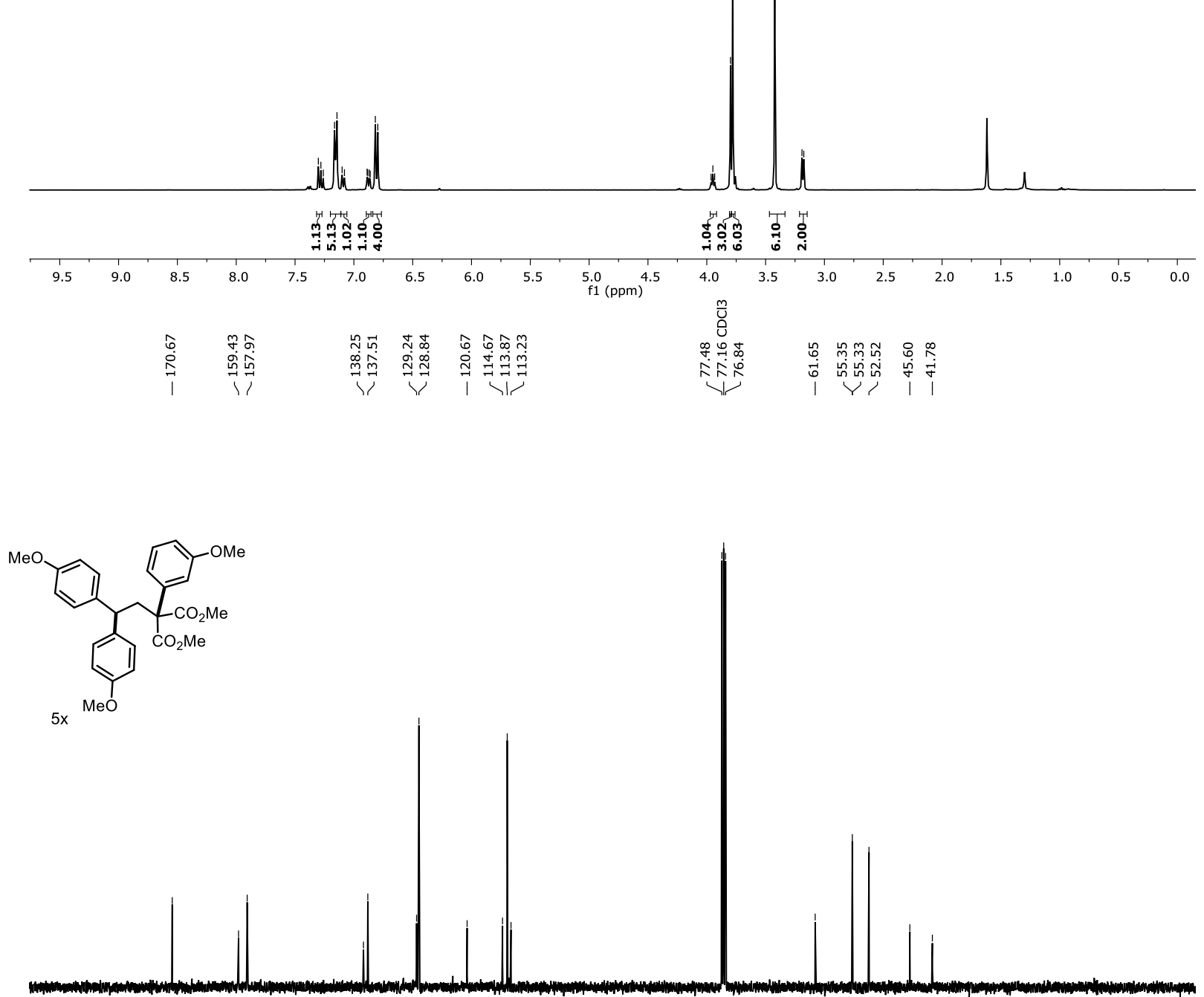

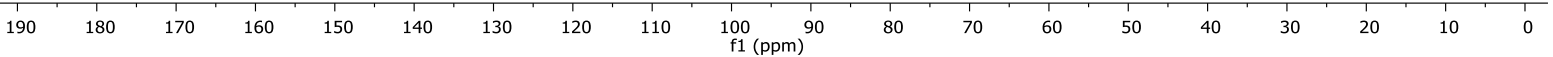




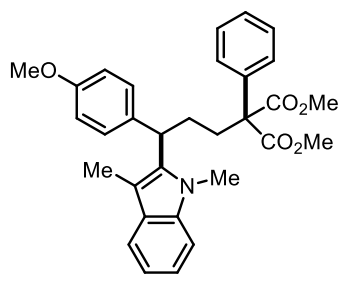

$7 a$

(400 MHz, $\mathrm{CDCl}_{3}$ )
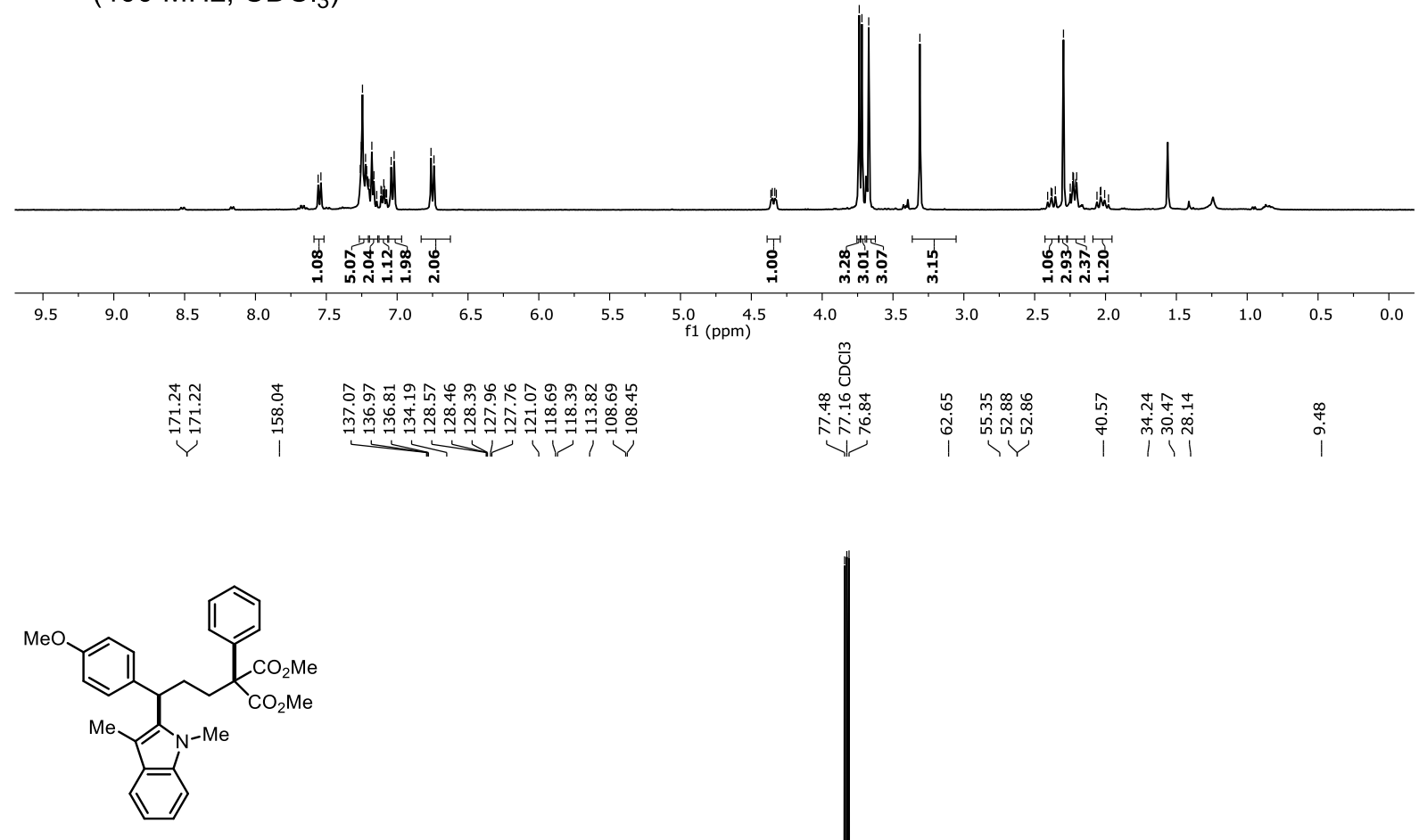

$7 a$

$\left(100 \mathrm{MHz}, \mathrm{CDCl}_{3}\right)$

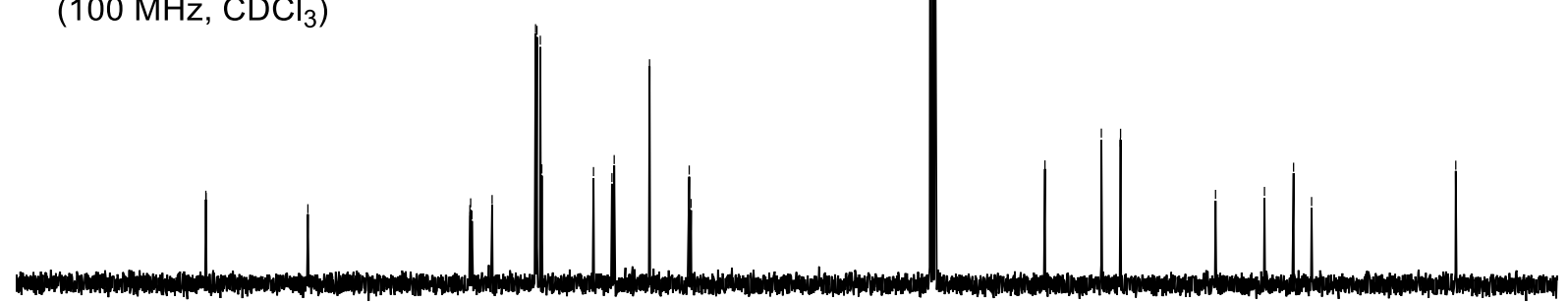

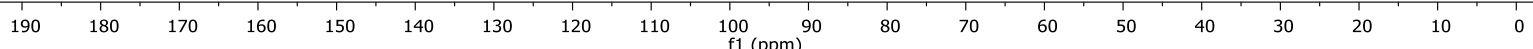




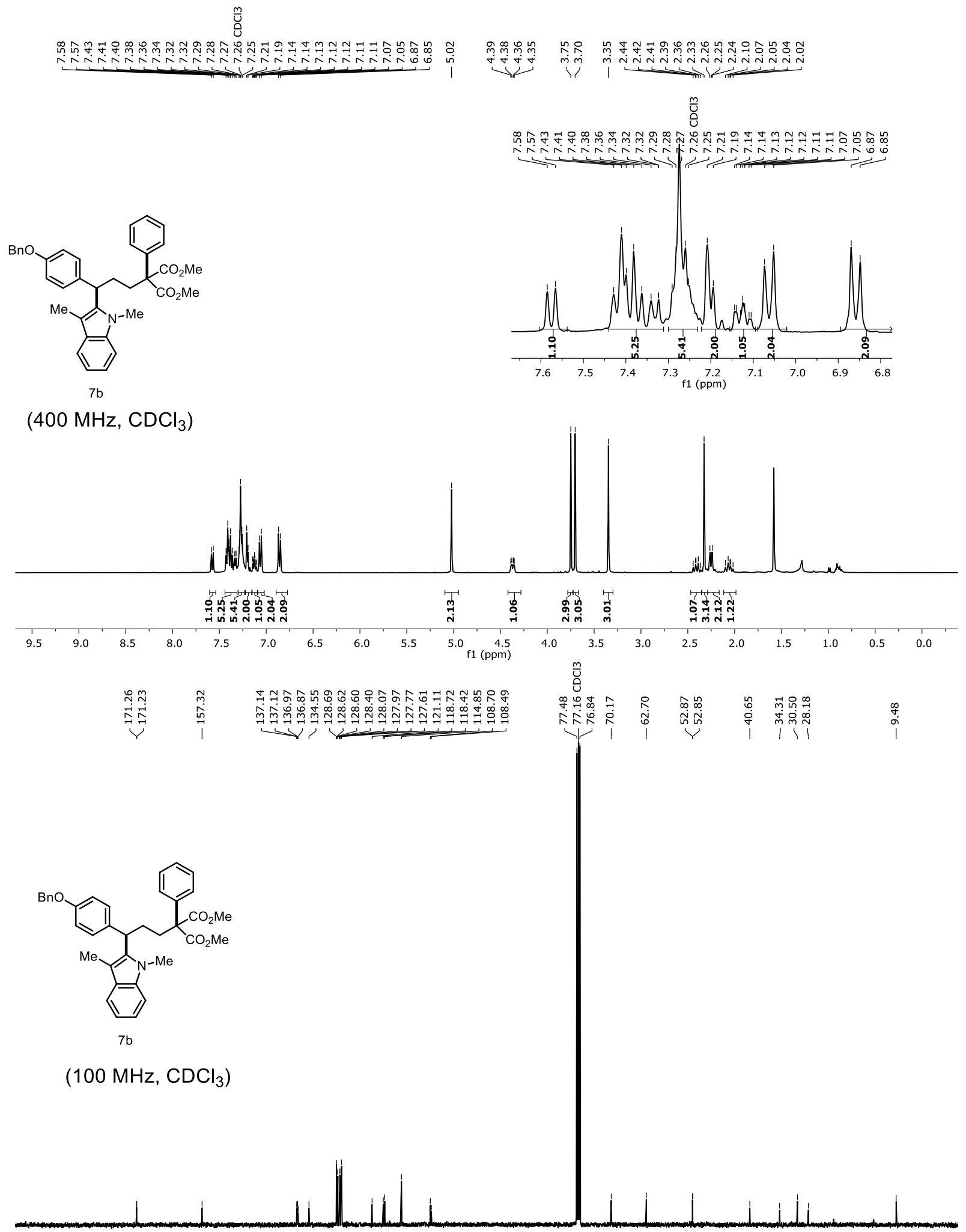

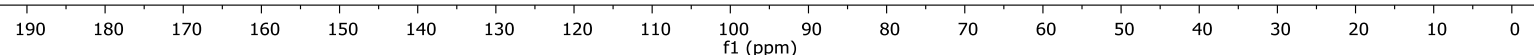




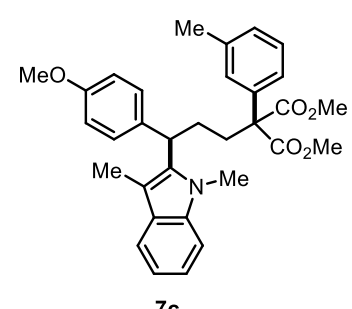

(400 MHz, $\mathrm{CDCl}_{3}$ )

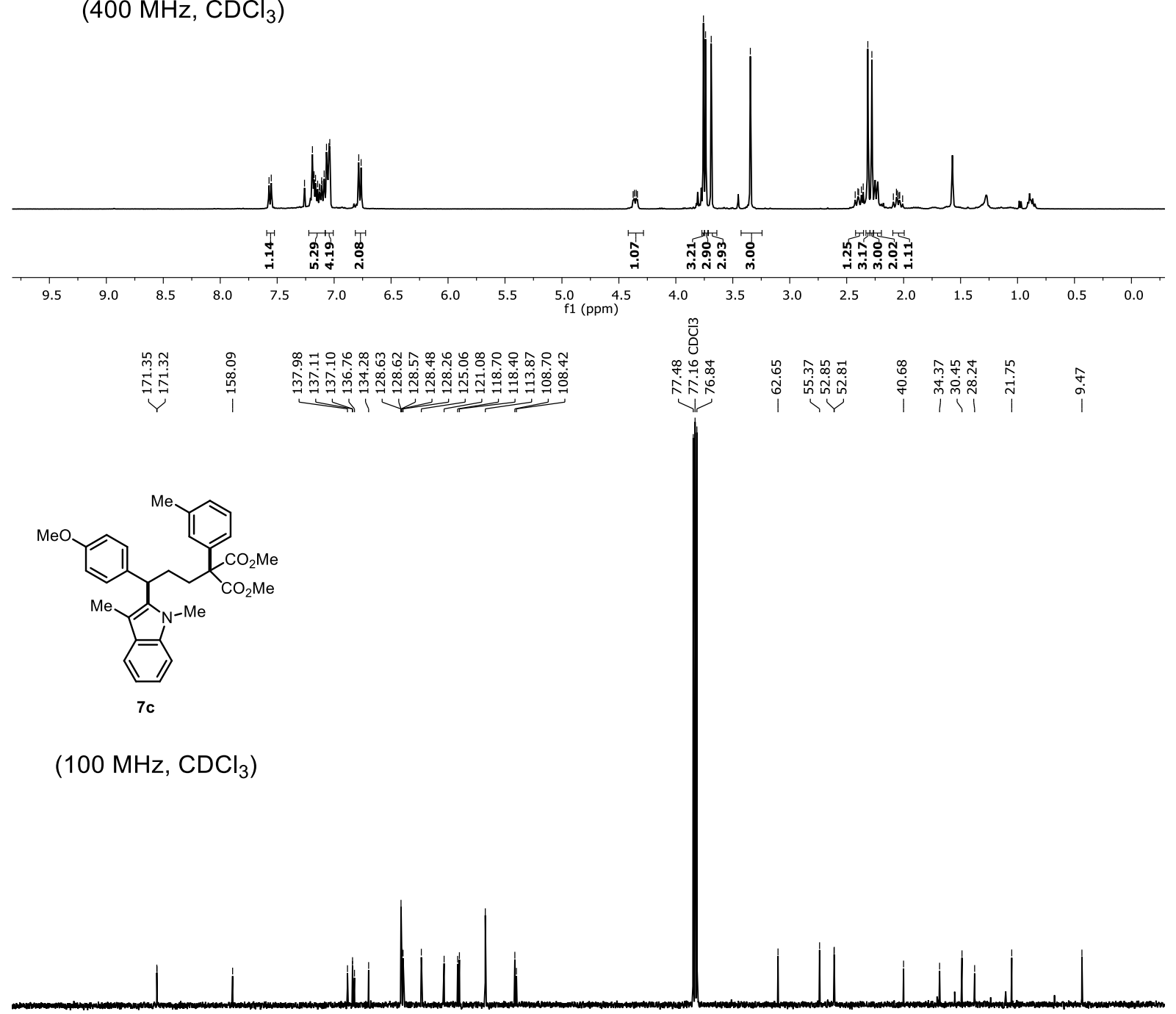

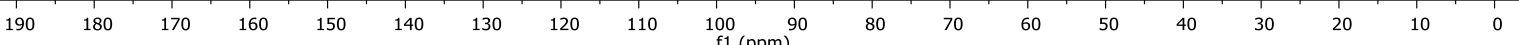


产

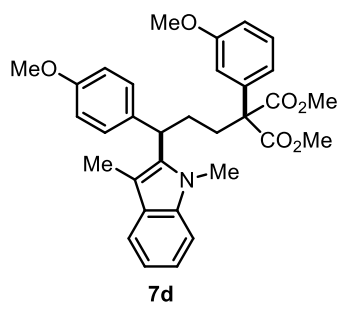

(400 $\mathrm{MHz}, \mathrm{CDCl}_{3}$ )
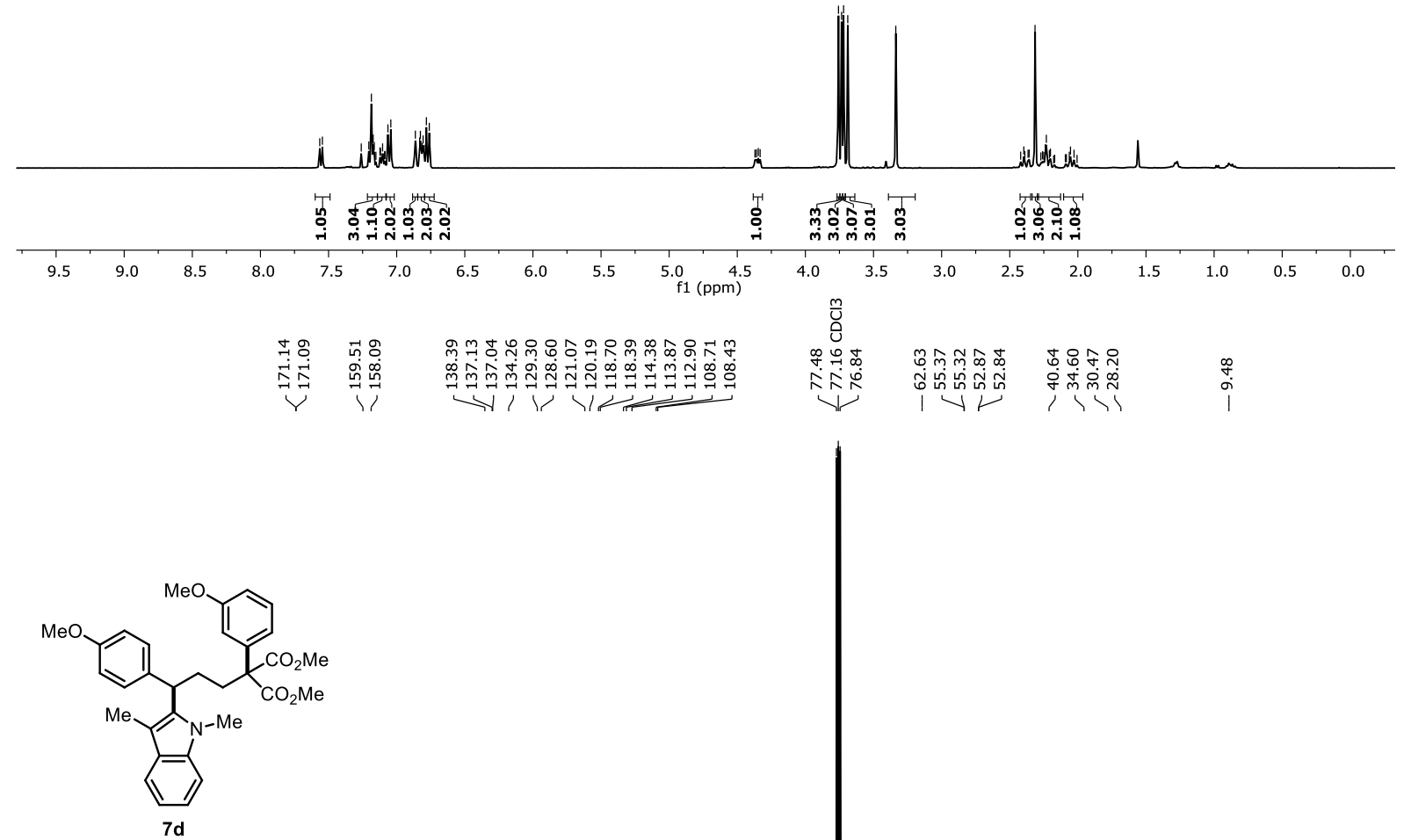

$\left(100 \mathrm{MHz}, \mathrm{CDCl}_{3}\right)$

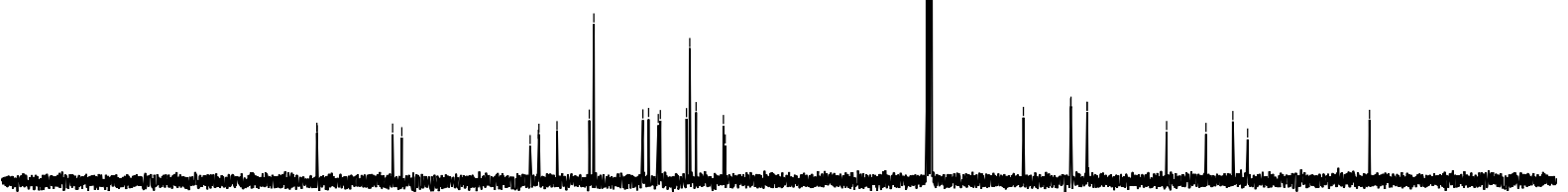

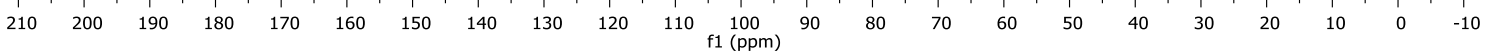




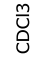

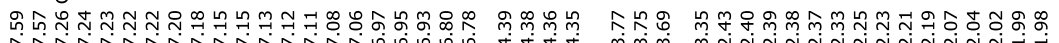

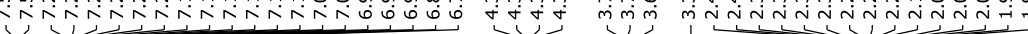<smiles>COC(=O)C(CCC(c1ccc(F)cc1)c1ccc(OC)cc1)(OC)c1c(C)c2ccccc2n1C</smiles>

$7 e$

(400 $\left.\mathrm{MHz} \mathrm{CDCl}_{3}\right)$
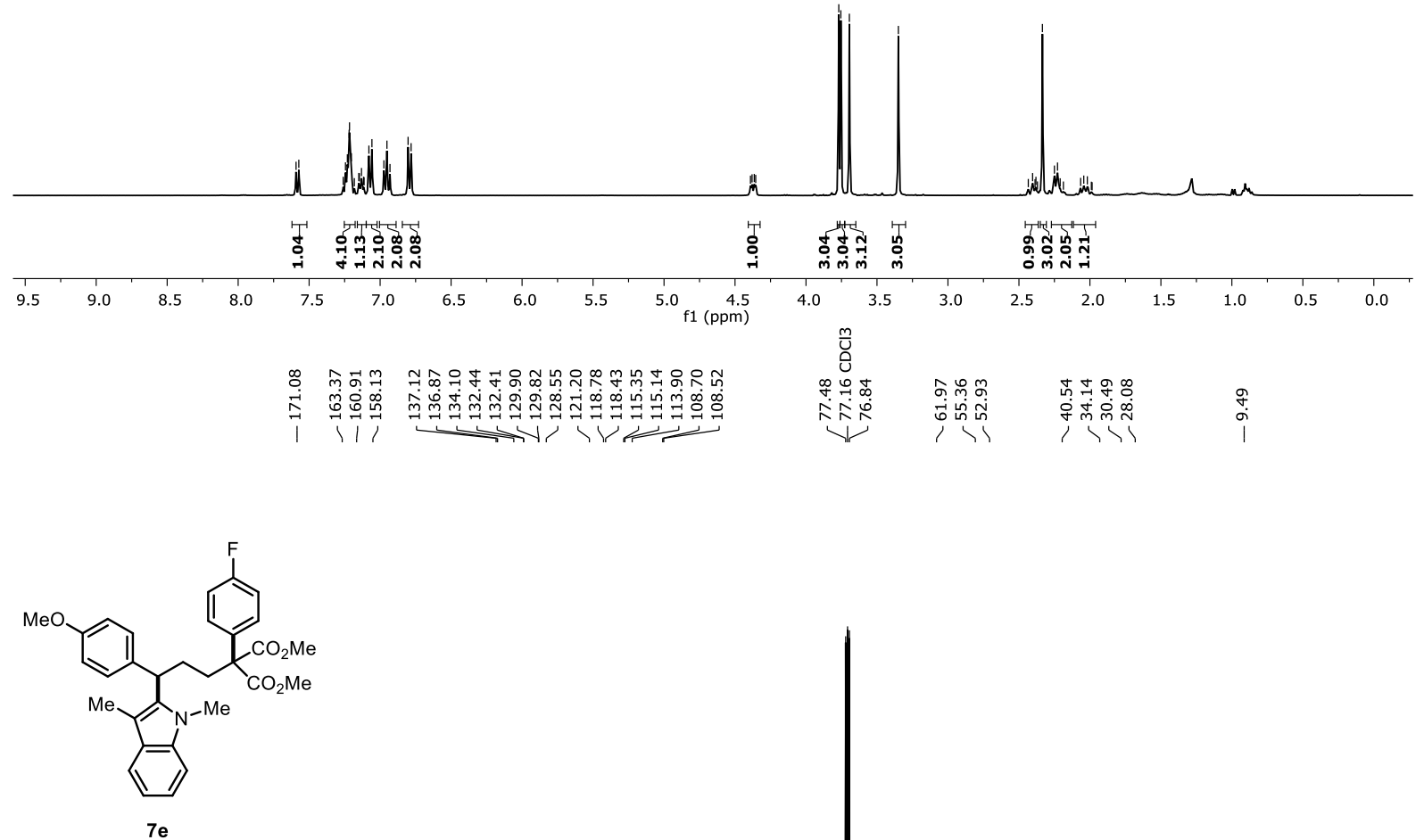

$\left(100 \mathrm{MHz}, \mathrm{CDCl}_{3}\right)$

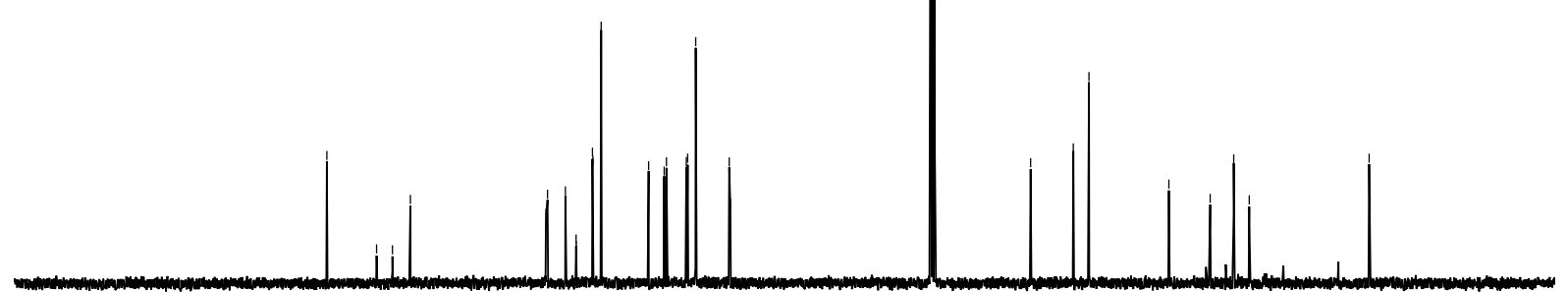

$\begin{array}{llllllllllllllllllllllllll}210 & 200 & 190 & 180 & 170 & 160 & 150 & 140 & 130 & 120 & 110 & \underset{f 1}{100}(\mathrm{ppm}) & 90 & 80 & 70 & 60 & 50 & 40 & 30 & 20 & 10 & 0 & -10\end{array}$ 


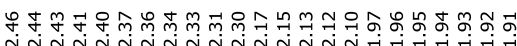

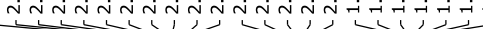

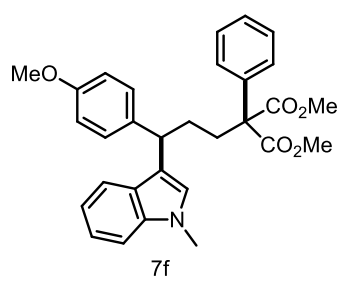

$\left(400 \mathrm{MHz}, \mathrm{CDCl}_{3}\right)$
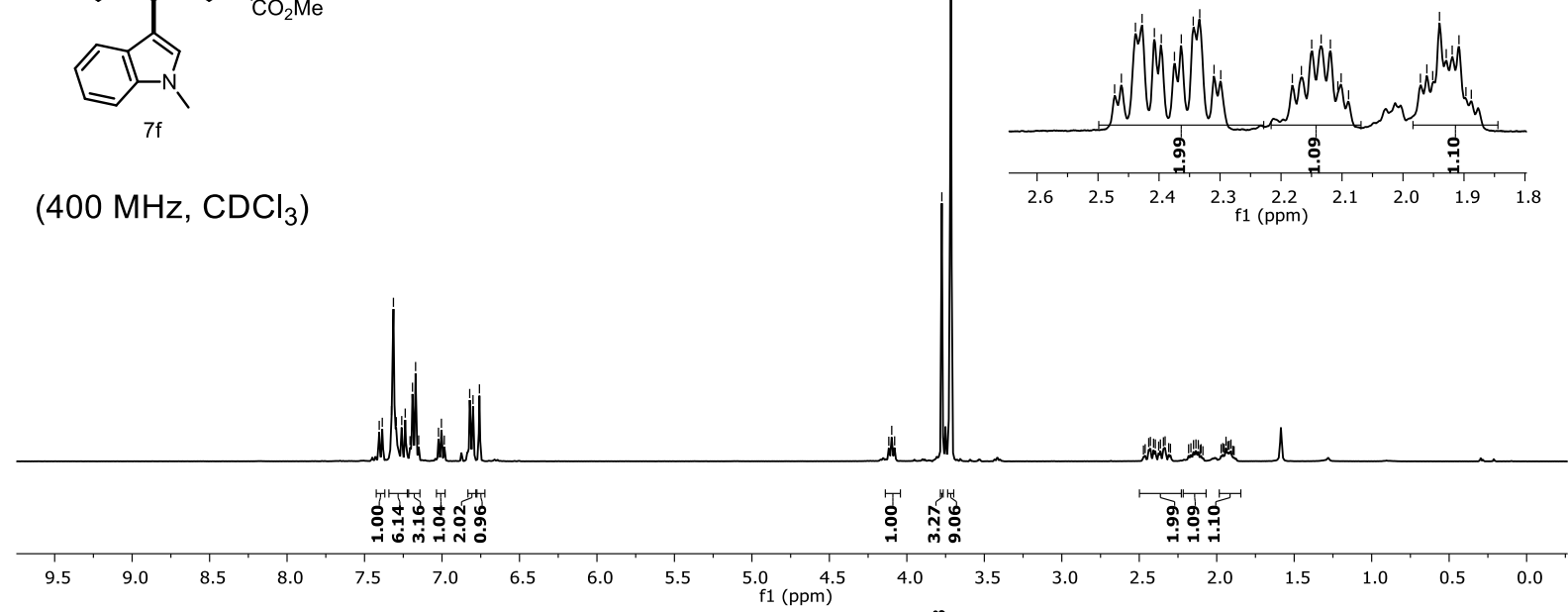

年<smiles>COc1ccc(C(CCC(C(C)=O)(C(C)=O)c2ccccc2)c2cn(C)c3ccccc23)cc1</smiles>

$\left(100 \mathrm{MHz} \mathrm{CDCl}_{3}\right)$

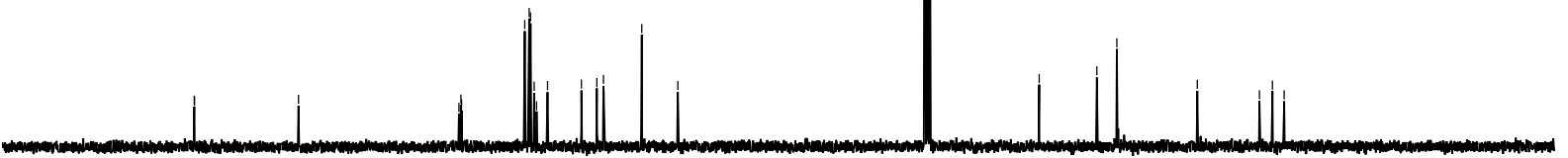

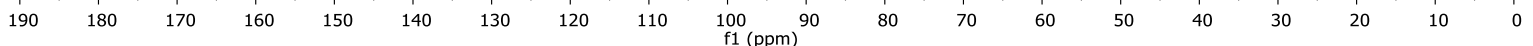


<smiles>COc1ccc(C(CCC(C)(OC)c2ccccc2)c2c(C)n(C)c3ccccc23)cc1</smiles>

$7 \mathrm{~g}$

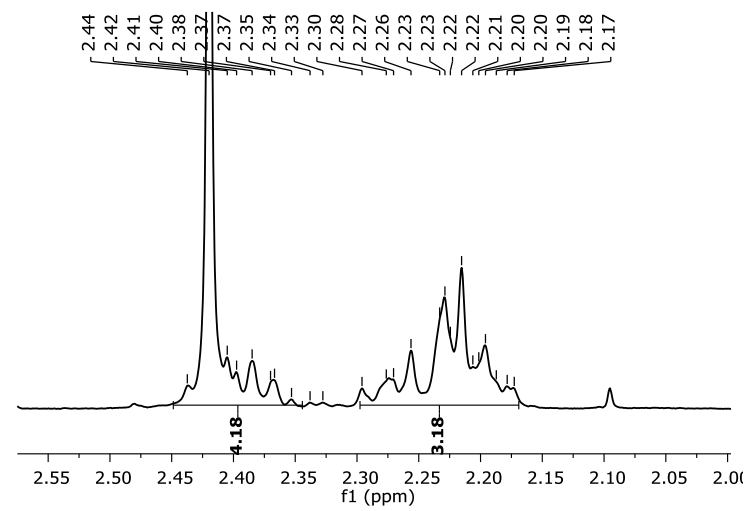

$\left(400 \mathrm{MHz}, \mathrm{CDCl}_{3}\right)$

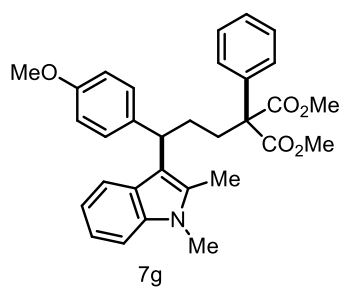

(100 MHz, $\mathrm{CDCl}_{3}$ )

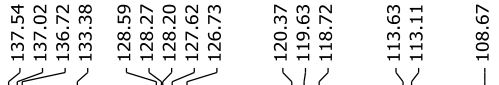

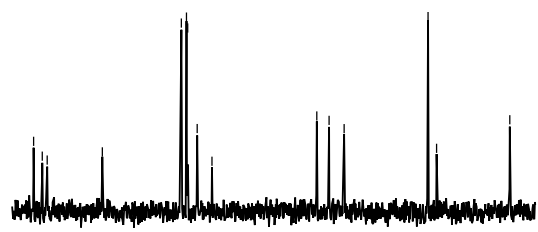

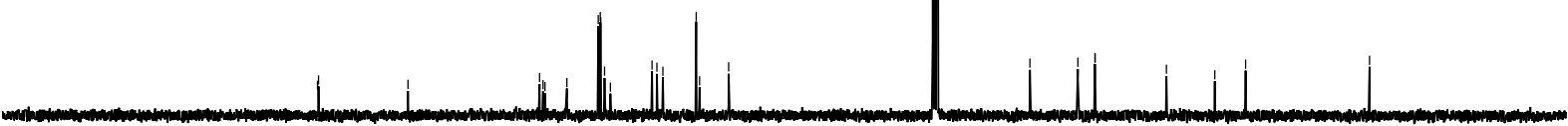

$\begin{array}{lllllllllllllllllllllll}210 & 200 & 190 & 180 & 170 & 160 & 150 & 140 & 130 & 120 & 110 & \begin{array}{c}100 \\ \mathrm{f} 1(\mathrm{ppm})\end{array} & 90 & 80 & 70 & 60 & 50 & 40 & 30 & 20 & 10 & 0 & -10\end{array}$ 


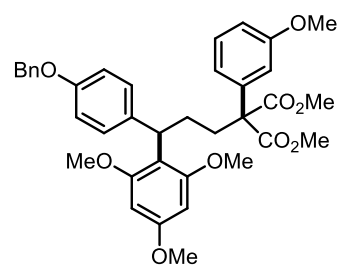

$7 \mathbf{h}$

(400 MHz, $\mathrm{CDCl}_{3}$ )
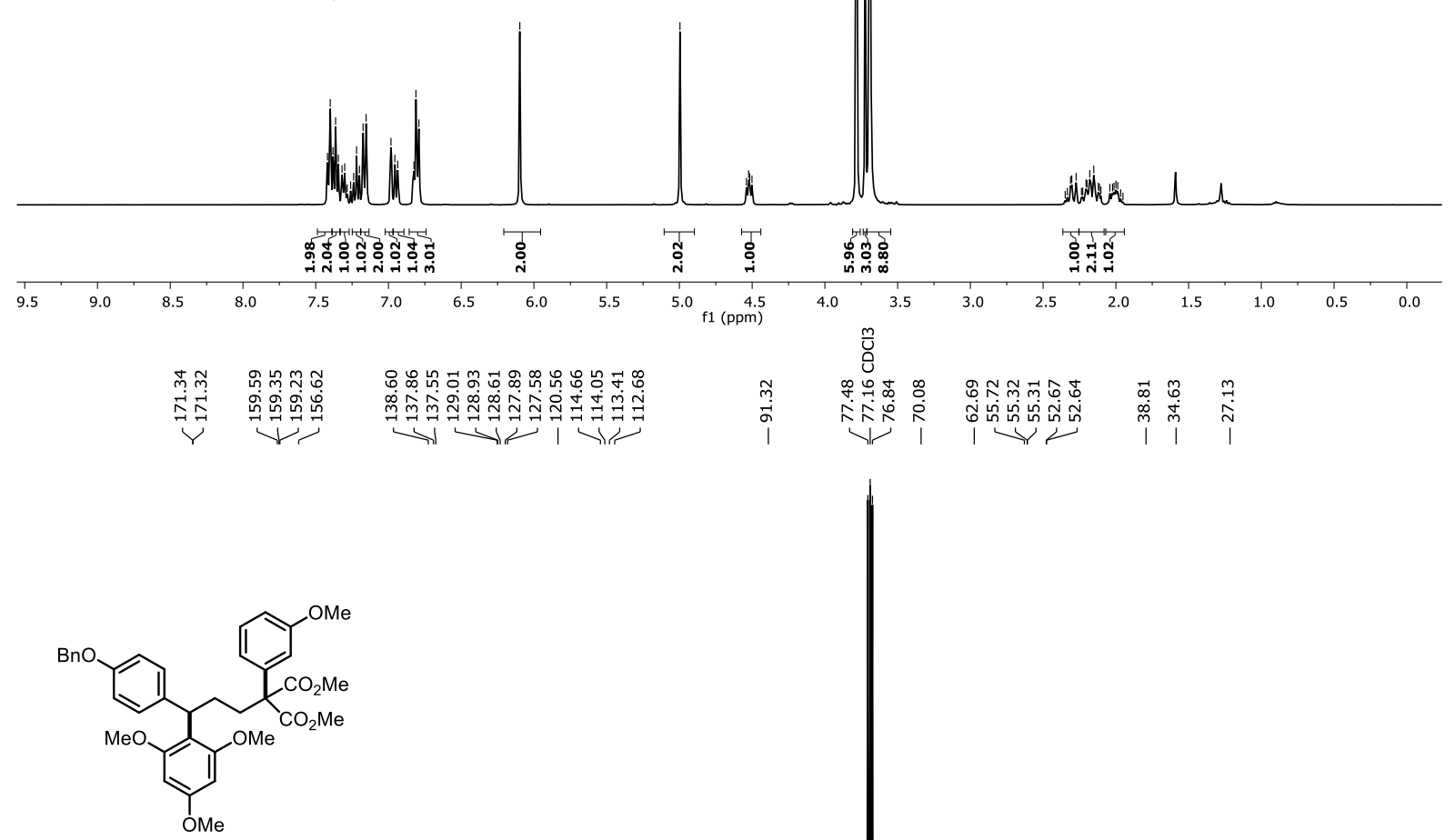

(100 MHz, $\mathrm{CDCl}_{3}$ )

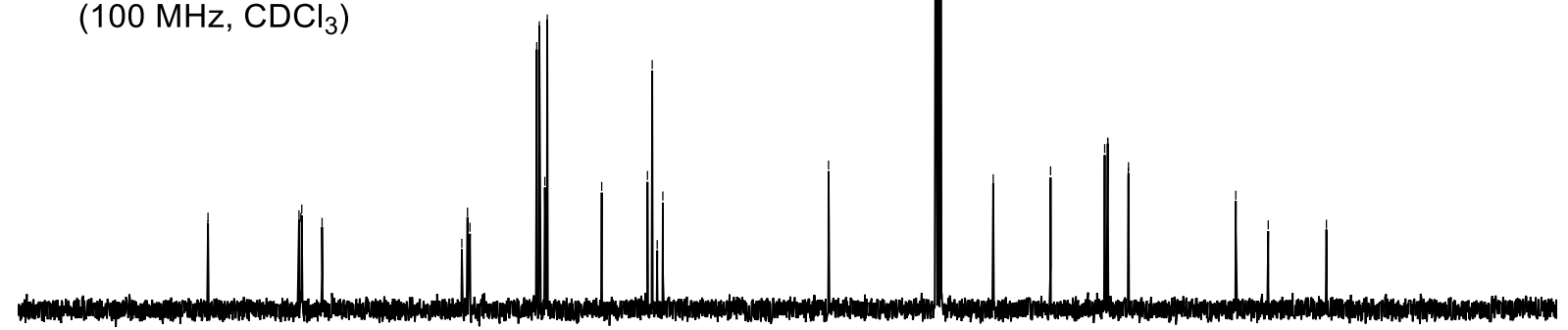

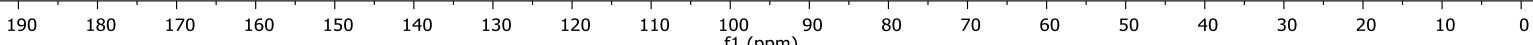




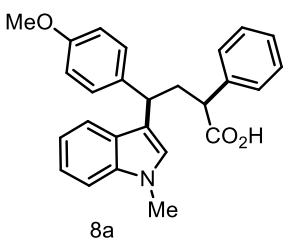

$\left(400 \mathrm{MHz}, \mathrm{CDCl}_{3}\right)$
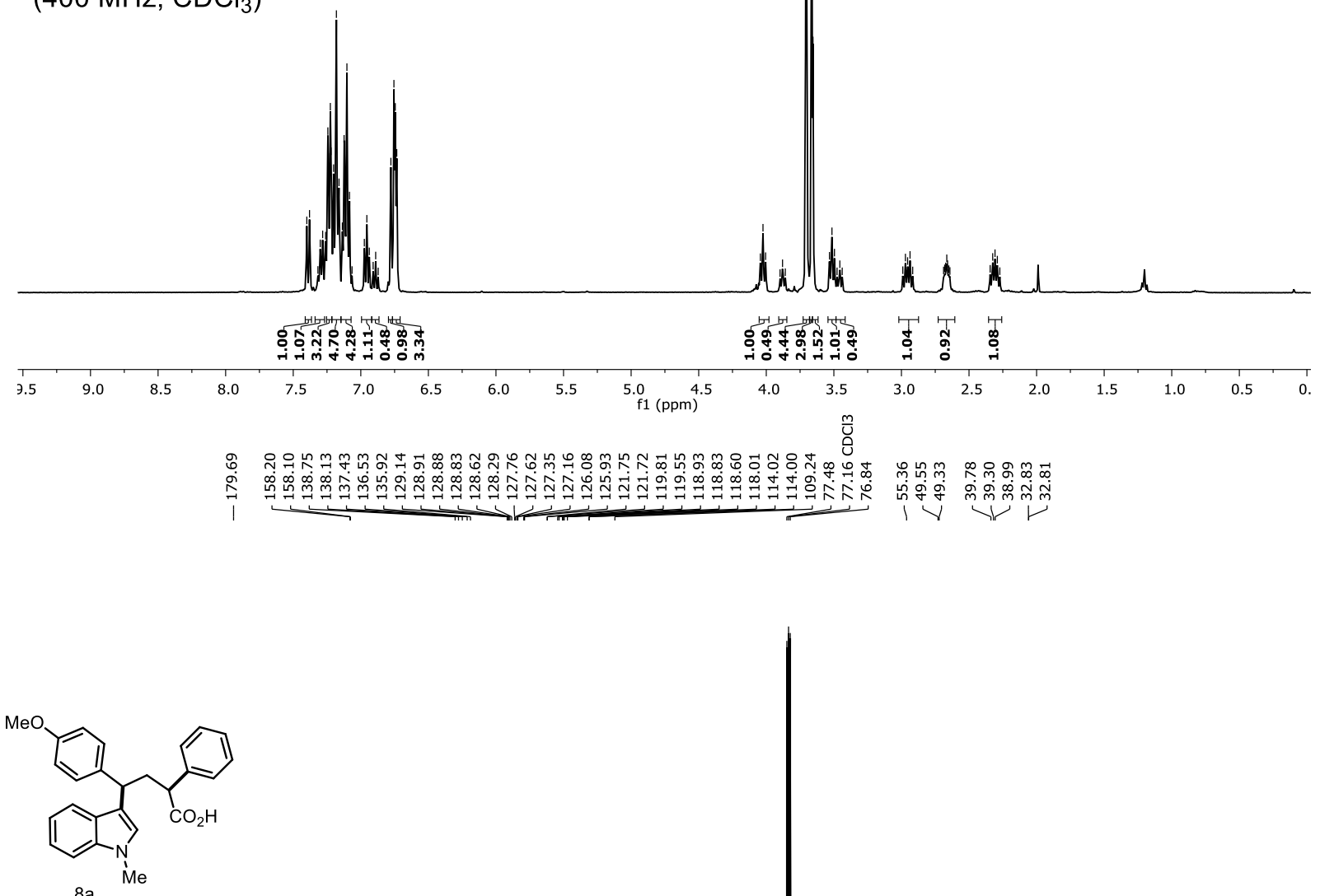

$\left(100 \mathrm{MHz}, \mathrm{CDCl}_{3}\right)$

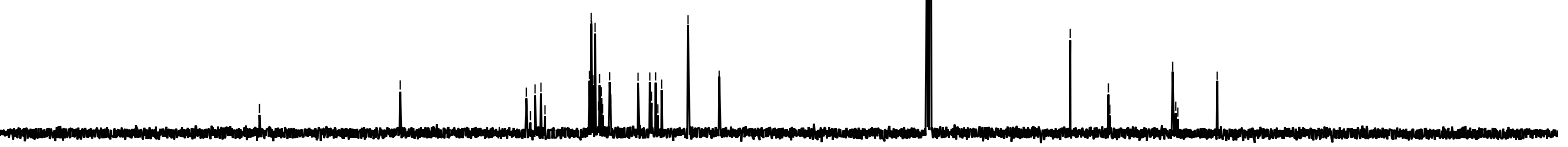

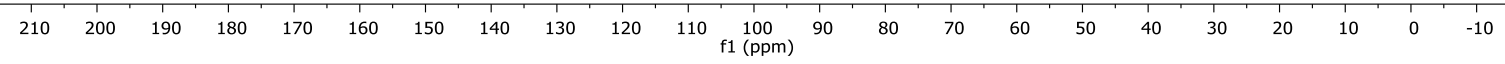




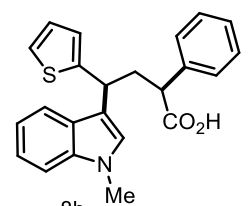

$8 \mathrm{~b} \dot{\mathrm{Me}}$

$\left(400 \mathrm{MHz}, \mathrm{CDCl}_{3}\right)$

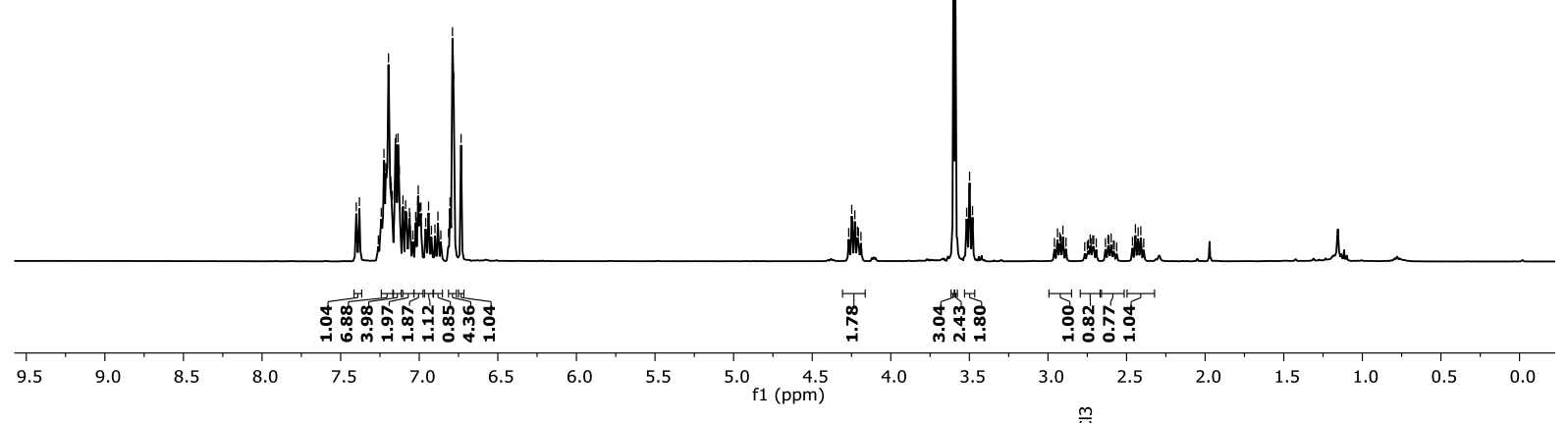

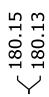

궁

守离

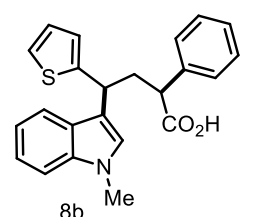

$\left(100 \mathrm{MHz} \mathrm{CDCl}_{3}\right)$

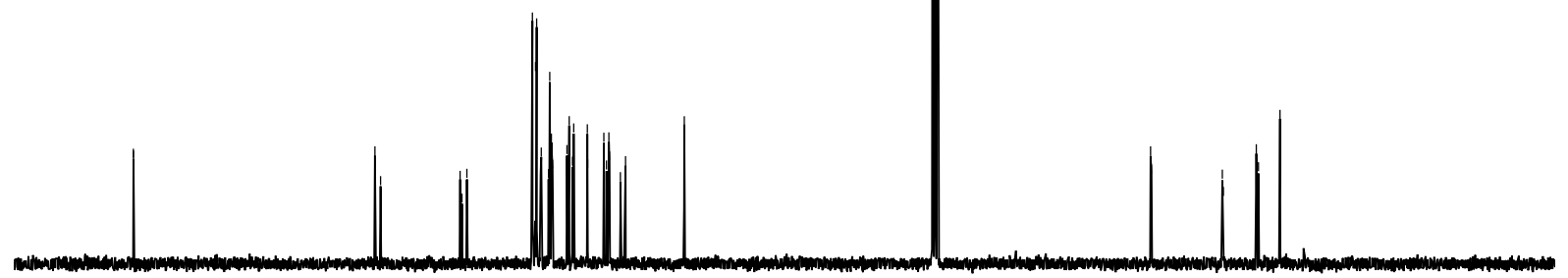

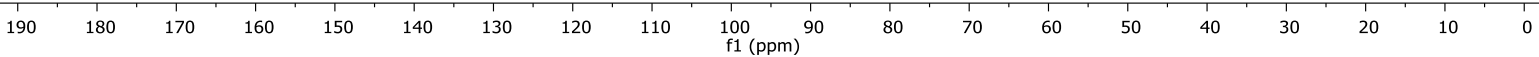


茜

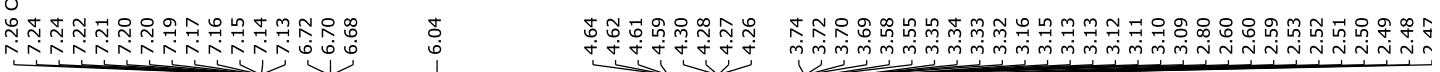<smiles>COc1ccc(C(c2ccccc2)C(Cc2ccccc2)c2cc(O)cc([N+](=O)[O-])c2)c(O)c1</smiles>

$\left(400 \mathrm{MHz}, \mathrm{CDCl}_{3}\right)$
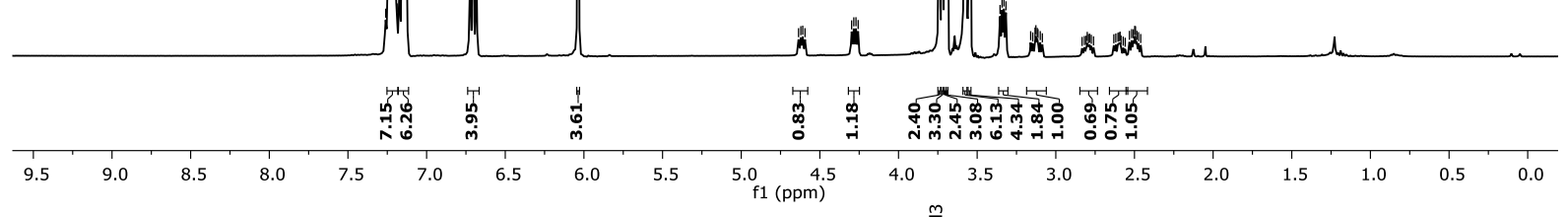

on $\quad$ 员

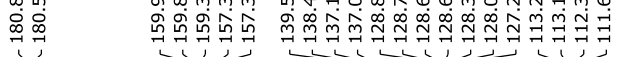

年

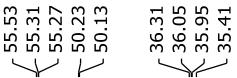

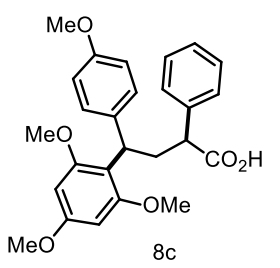

(100 MHz, $\mathrm{CDCl}_{3}$ )

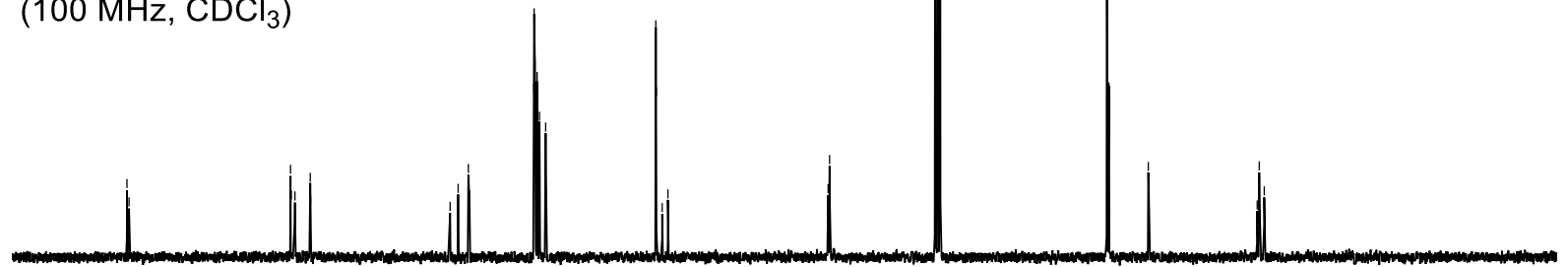

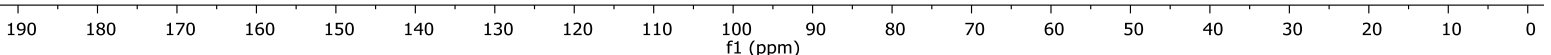




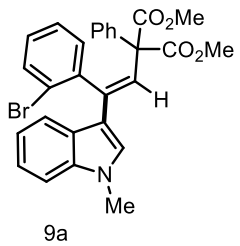

(400 MHz, $\mathrm{CDCl}_{3}$ )
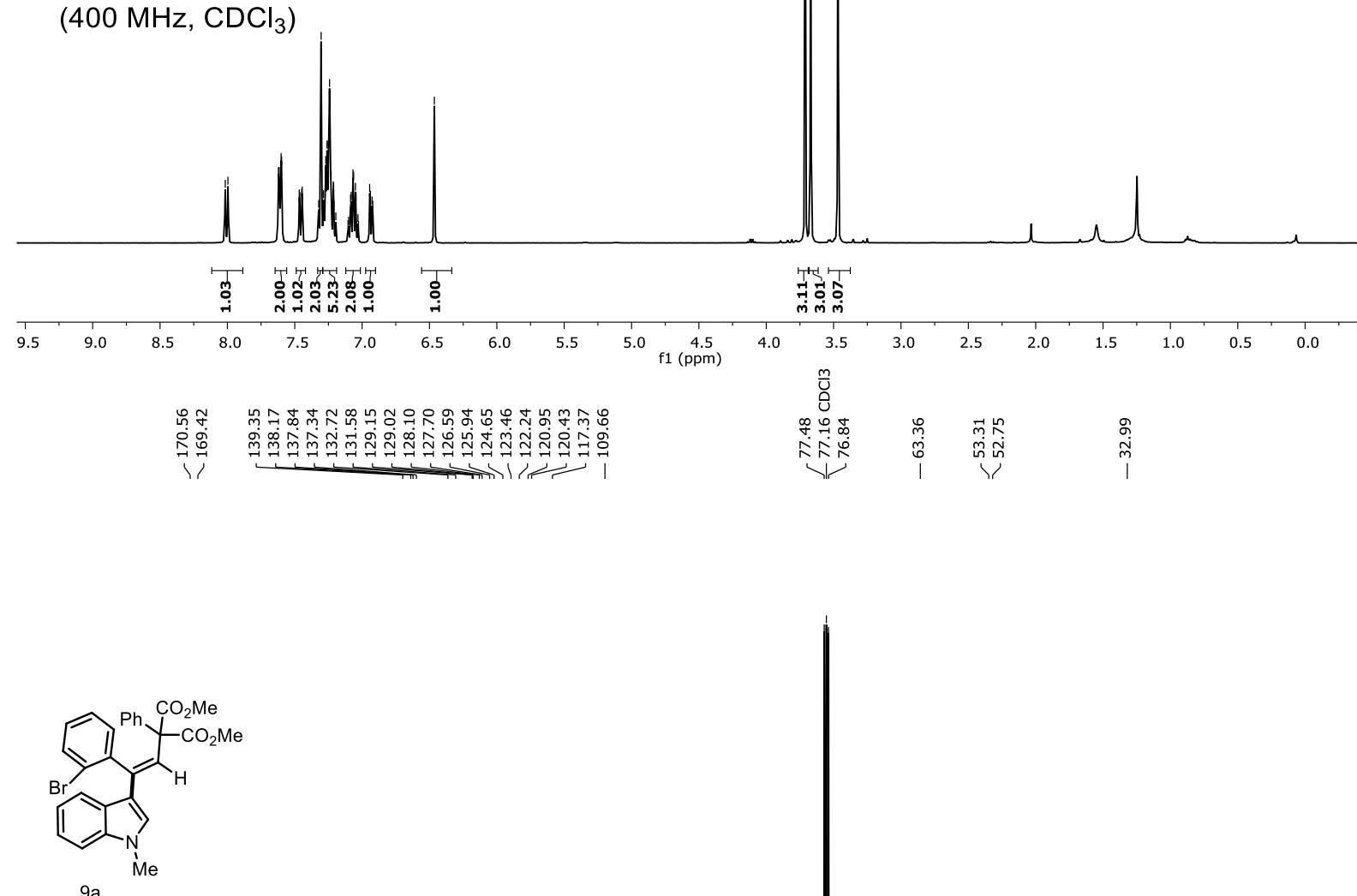

$9 a$

(100 MHz, $\mathrm{CDCl}_{3}$ )

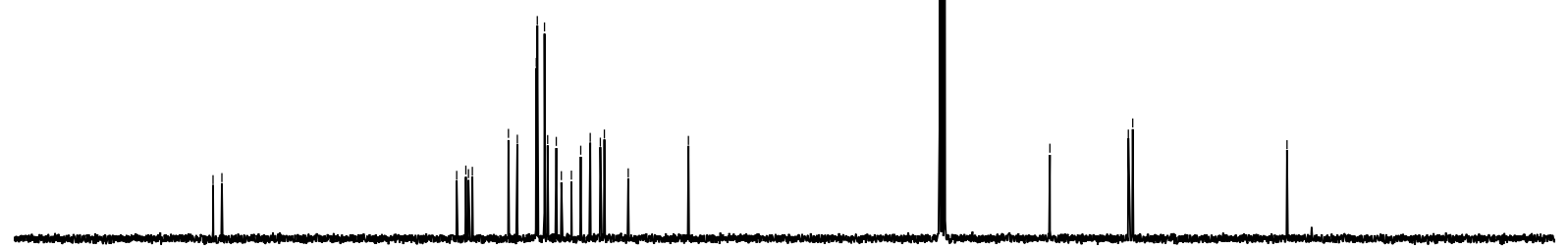

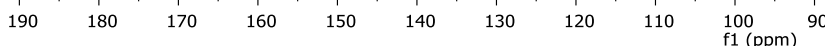




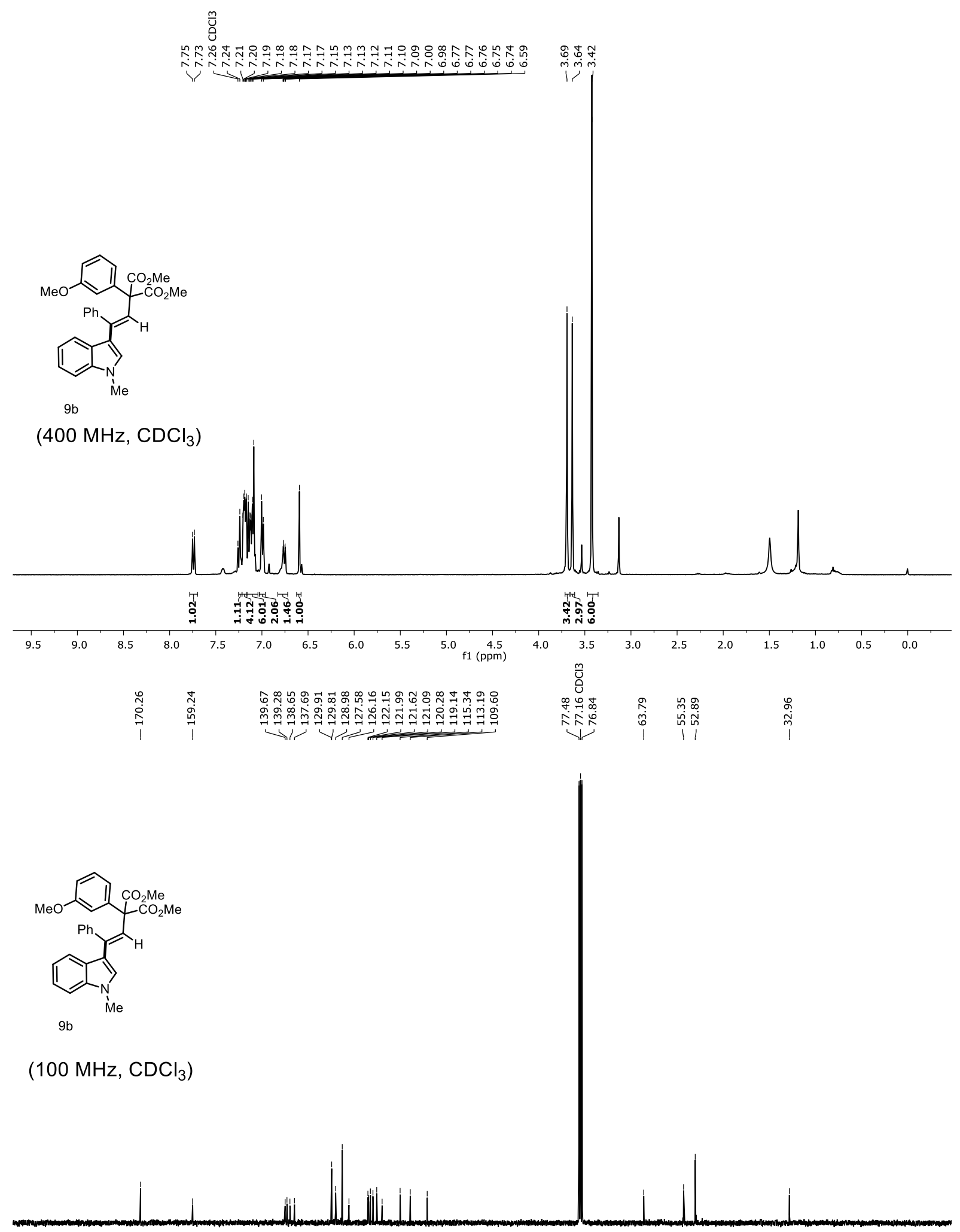

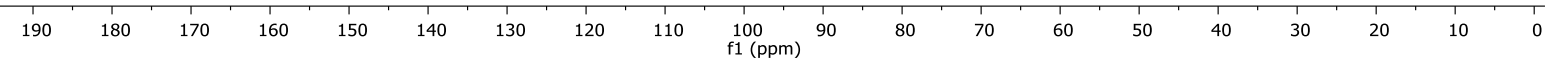




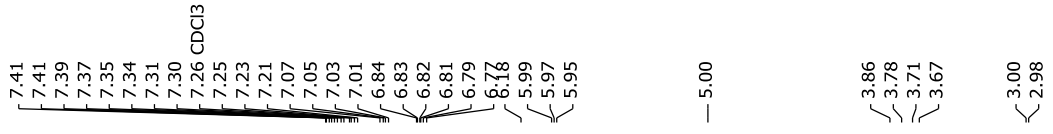<smiles>COc1cccc(/C(=C\CC(OC)(OC)c2cccc(OC)c2)c2c(OC)cc(OC)cc2OC)c1</smiles>

$\left(400 \mathrm{MHz}, \mathrm{CDCl}_{3}\right)$
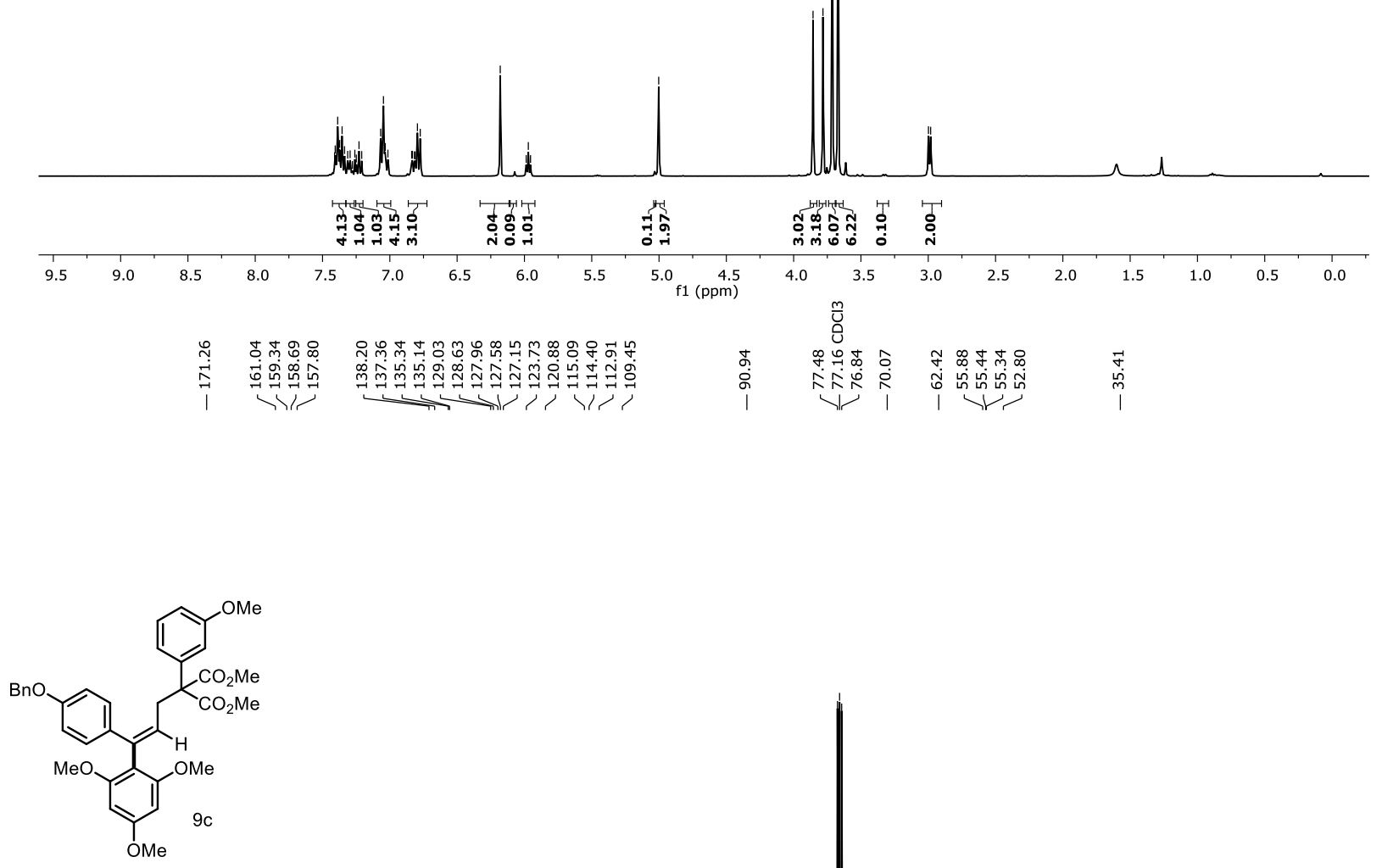

$\left(100 \mathrm{MHz}, \mathrm{CDCl}_{3}\right)$

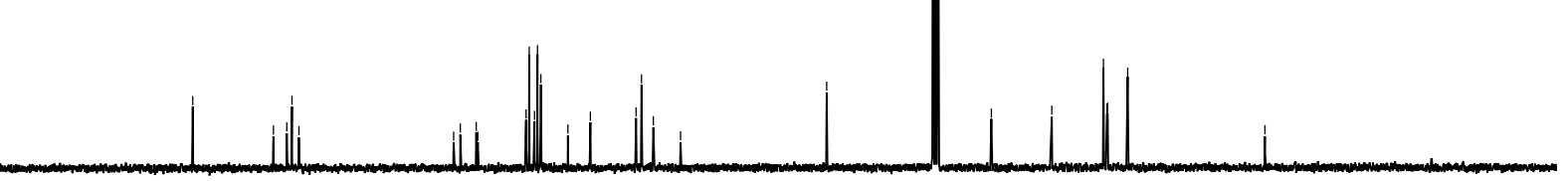

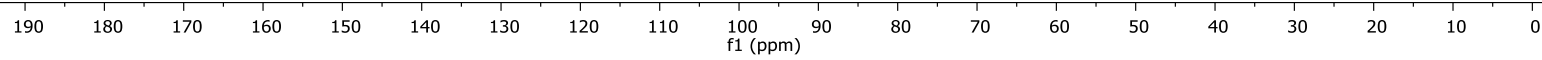




\section{Crystallographic Data}

Compound 5a was crystalized from the mixture of solvent $\mathrm{CH}_{2} \mathrm{Cl}_{2}$ : $\mathrm{MeOH}(100: 1)$ via slow evaporation. Crystal structures of $\mathbf{5 a}$ was determined on Bruker D8 Quest equipped with a micro-focus source to generate Mo Ka radiation $(\lambda=0.71073 \AA)$ and a PHOTON II CMOS detector. Data were collected at 298K. Integration and scaling of data were performed by SAINT ${ }^{1}$ and SADABS program ${ }^{2}$ respectively. The structures were solved by direct methods using SHELXT-2018 ${ }^{3}$ and refined by fullmatrix least-squares on $\mathrm{F}^{2}$ using SHELXL-2018/3 version ${ }^{3}$. All non-hydrogen atoms were refined anisotropically and all hydrogen atoms were placed at calculated positions using riding models.

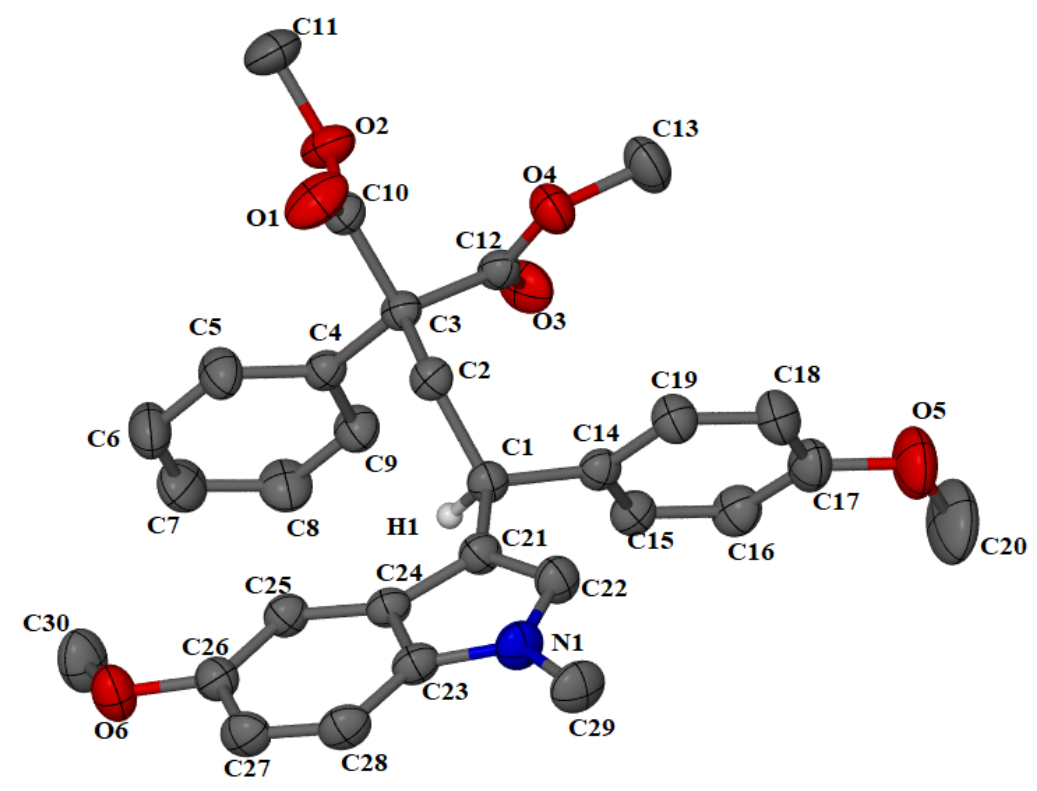

ORTEP diagram of 5a (CCDC No 2003418) : Atoms are shown with $30 \%$ probability of thermal ellipsoids

Table S2. Crystal data and refinement parameters

\begin{tabular}{|l|l|}
\hline Code & $\mathbf{5 a}$ \\
\hline Empirical formula & $\mathrm{C}_{30} \mathrm{H}_{31} \mathrm{~N}_{1} \mathrm{O}_{6}$ \\
\hline Formula weight & 501.56 \\
\hline Wavelength/ $\AA$ & 0.71073 \\
\hline Crystal system & triclinic \\
\hline Space group & $P-1$ \\
\hline Crystal size $\left(\mathrm{mm}^{3}\right)$ & $0.35 \times 0.25 \times 0.11$ \\
\hline
\end{tabular}




\begin{tabular}{|c|c|}
\hline$a / \AA$ & $7.5036(6)$ \\
\hline$b / \AA$ & 10.9282(10) \\
\hline$c / \AA$ & 16.4574(15) \\
\hline$\alpha /\left(^{\circ}\right)$ & $77.389(3)$ \\
\hline$\beta /\left(^{\circ}\right)$ & $79.887(3)$ \\
\hline$y /\left(^{\circ}\right)$ & $85.461(3)$ \\
\hline$V / \AA^{3}$ & 1295.3(2) \\
\hline Z & 2 \\
\hline$D_{\text {cal }} / \mathrm{g} \mathrm{cm}^{-3}$ & 1.286 \\
\hline $\mathrm{T} / \mathrm{K}$ & 298 \\
\hline$\mu / \mathrm{mm}^{-1}$ & 0.089 \\
\hline$F_{000}$ & 532 \\
\hline Theta ranges for data collection & $2.5^{\circ}$ to $28.3^{\circ}$ \\
\hline Index ranges & $\begin{array}{l}-9 \leq \mathrm{h} \leq 9, \quad-14 \leq \mathrm{k} \leq 14, \quad-21 \leq \mathrm{I} \leq \\
21\end{array}$ \\
\hline Reflections measured & 36007 \\
\hline Unique reflections & 6372 \\
\hline Observed reflections & 5061 \\
\hline Parameters & 339 \\
\hline Data completeness & 0.992 \\
\hline$R_{\text {int }}$ & 0.045 \\
\hline final $R(I>2 \sigma(I))$ & 0.0469 \\
\hline final $R$ (all data) & 0.0612 \\
\hline final $w R_{2}(I>2 \sigma(I))$ & 0.1234 \\
\hline final $w R_{2}$ (all data) & 0.1333 \\
\hline
\end{tabular}




\begin{tabular}{|l|l|}
\hline GOF on $\mathrm{F}^{2}$ & 1.026 \\
\hline Highest peak and deepest hole & $0.22 \&-0.17$ \\
\hline CCDC No. & 2003418 \\
\hline
\end{tabular}

References:

1. SAINT, Version 6.45; Bruker AXS Inc.: Madison, WI, 2003.

2. SADABS, Version 2.05; Bruker AXS Inc.: Madison, WI, 2002.

3. Sheldrick, G. M. Acta Cryst. Sect. A. 2015, 71, 3-8 
1. (a) Goldberg, A. F. G.; O'Connor, N.R.; Craig, R. A.; Stoltz, B. M. Org. Lett. 2012, 14, 5314. (b) Singh, K.; Bera, T.; Jaiswal, V.; Biswas, S.; Mondal, B.; Das, D.; Saha, J. J. Org. Chem. 2019, 84, 710. (c) Ghosh, A.; Mandal, S.; Chattaraj, P.K.; Banerjee, P. Org. Lett. 2016, 18, 4940. (d) Blanchard, A.L.; Schneider, J. A. J. Org. Chem. 1986, 51, 1372. (e) Pavlova, A. S.; Ivanova, O. A.; Chagarovskiy, A.O.; Stebunov, N.S.; Orlov, N.V.; Shumsky, A.N.; Budynina, E.M.; Rybakov, V. B.; Trushkov, I. V. Chem. Eur. J. 2016, 22, 17967. (f) Augustin, A. U.; Busse, M.; Jones, P. G.; Werz, D. B. Org. Lett. 2018, 20, 820. (g) Ivanov, K. L.; Villemson, E. V.; Budynina, E. M.; Ivanova, O. A.; Trushkov, I. V.; Melnikov, M. Y. Chem. Eur. J. 2015, 21, 4975. (h) Ma, H.; Hu, X.-Q.; Luo, Y.-C.; Xu, P.-F. Org. Lett. 2017, 19, 6666. (i) Lin, S.; Li, M.; Dong, Z.; Liang, F.; Zhang, J. Org. Biomol. Chem. 2014, 12, 1341.

2. (a) Moustafa, M. M A. R., Pagenkopf, B. L. Org. Lett. 2010, 12, 4732.(b) Hu, J.-L.; Feng, L.-W.; Wang, L.;Xie, Z.; Tang, Y.; Li, X. J. Am. Chem. Soc. 2016, 138, 13151.

3. (a) Rahman, A. F. M. M.; Murafuji, T.; Ishibashi, M.; Miyoshi,Y.; Sugihara, Y.J. Organomet, Chem. 2004, 689, 3395. (b) Goel, R.G.; Prasad, H. S. Can. J. Chem. 1971, 49, 2529. (c) Koech, P. K.; Krische, M. J. J. Am. Chem. Soc. 2004, 126, 5350. (d) Matano, Y.; Nomura, H.; Suzuki, H.; Shiro, M.; Nakano, H. J. Am.Chem. Soc. 2001, 123, 10954. (e) Urano, M.; Wada, S.; Suzuki, H. Chem. Commun. 2003, 1202. 